\title{
Modeling of PMS Ae/Fe stars using UV spectra ${ }^{\star}, \star \star$
}

\author{
P. F. C. Blondel ${ }^{1,2}$ and H. R. E. Tjin A Djie ${ }^{1}$ \\ 1 Astronomical Institute "Anton Pannekoek", University of Amsterdam, Kruislaan 403, 1098 SJ Amsterdam, The Netherlands \\ e-mail: blondel@sara.nl \\ 2 SARA, Kruislaan 415, 1098 SJ Amsterdam, The Netherlands
}

Received 13 February 2004 / Accepted 13 October 2005

\begin{abstract}
Context. Spectral classification of PMS Ae/Fe stars, based on visual observations, may lead to ambiguous conclusions. Aims. We aim to reduce these ambiguities by using UV spectra for the classification of these stars, because the rise of the continuum in the UV is highly sensitive to the stellar spectral type of A/F-type stars.

Methods. We analyse the low-resolution UV spectra in terms of a 3-component model, that consists of spectra of a central star, of an optically-thick accretion disc, and of a boundary-layer between the disc and star. The disc-component was calculated as a juxtaposition of Planck spectra, while the 2 other components were simulated by the low-resolution UV spectra of well-classified standard stars (taken from the IUE spectral atlases). The hot boundary-layer shows strong similarities to the spectra of late-B type supergiants (see Appendix A).

Results. We modeled the low-resolution UV spectra of 37 PMS Ae/Fe stars. Each spectral match provides 8 model parameters: spectral type and luminosity-class of photosphere and boundary-layer, temperature and width of the boundary-layer, disc-inclination and circumstellar extinction. From the results of these analyses, combined with available theoretical PMS evolutionary tracks, we could estimate their masses and ages and derive their mass-accretion rates. For a number of analysed PMS stars we calculated the corresponding SEDs and compared these with the observed SEDs.

Conclusions. All stars (except $\beta$ Pic) show indications of accretion, that affect the resulting spectral type of the stellar photosphere. Formerly this led to ambiguities in classificaton of PMS stars as the boundary-layer was not taken into consideration. We give evidence for an increase of the mass-accretion rate with stellar mass and for a decreases of this rate with stellar age.
\end{abstract}

Key words. stars: pre-main sequence - accretion, accretion disks

\section{Introduction}

During the past decades it has become clear that low-mass stars $\left(M_{\star}<1.0 M_{\odot}\right)$ in their PMS evolutionary stage, known as "classical" T Tauri stars (cTTs), are generally surrounded by accretion discs, which the contraction models of a rotating protostar predict. This has raised the question of whether more massive stars $\left(1.0 M_{\odot}<M_{\star}<4.0 M_{\odot}\right)$ that radiate strongly in the UV and thus are able to ionise the infalling material and even reverse the infall of material, have a similar PMS evolutionary phase, in particular, if Herbig Ae/Be stars also feature an accretion disc.

Herbig stars are usually intrinsically brighter than $\mathrm{T}$ Tauri stars, which makes them observable at larger distances and larger foreground extinction. However, because of their higher masses and therefore faster evolution, they are less numerous than the T Tauri stars.

So far, various indications of matter falling towards a Herbig Ae/Be star have been found from high-resolution spectroscopy in the visual (Hamann \& Persson 1992; Graham 1992; Tambotseva et al. 2001) and in the UV (Blondel et al. 1993; Pérez et al. 1993; Brown et al. 1997; Tjin A Djie et al. 1998;

* Based on observations by the International Ultraviolet Explorer collected at the Villafranca Satellite Tracking Station of the European Space Agency, on observations made by the Hipparcos Astrometry Satellite, and on ground-based photometric and spectroscopic observations made at the European Southern Observatory, La Silla, Chile.

$\star \star$ Figures 4-9, 33-40 and 45-110 and Appendices A-G are only available in electronic form at http://www.edpsciences.org
Valenti et al. 2000). Hillenbrand et al. (1992) have successfully fitted the visual and near-infrared (NIR) spectral energy distributions (SEDs) of a large sample of Herbig Ae/Be stars with a model consisting of a stellar photosphere and a flat, opticallythick accretion disc with an optically-thin circular central hole. These authors did not include the contribution of an accreting boundary-layer to the calculated SED.

The larger brightness of the PMS Ae/Fe stars makes it easier to obtain well-exposed (UV) spectra than for T Tauri stars. We analysed the low-resolution UV spectra, obtained with IUE in both the short (SW) and long (LW) wavelength range (Appendix F), of a sample of PMS Be/Ae/Fe stars (TWP94) in terms of a 3-component model (UV3C hereafter) that consists of a star, an accretion disc, and a boundary-layer (BL hereafter), through which the disc interacts with the star. The formalism of the model is given in Sect. 2, and in Sect. 3 we discuss the normalisation and the extinction corrections needed before we can compare the model spectrum to the observed spectrum.

In Sect. 4 we describe the procedure for matching the model spectrum to the observed spectrum and thus of determining the model parameters. Input values for these parameters were obtained from Appendices B-D and from Table 1. In this section we also show the sensitivity of the model spectrum to variations in the various model parameters. An important result is that in the IUE range the model spectrum receives no significant direct contributions from the disc component, so that our analysis only models the photosphere and the BL. Indirectly, however, the disc 
plays a role by shadowing the photospheric contribution, which justifies the use of the name UV3C.

Section 5 presents the results of the UV spectral analysis of $35 \mathrm{PMS} \mathrm{Be} / \mathrm{Ae} / \mathrm{Fe}$ stars and 2 cTTs. All stars (with the possible exception of $\beta$ Pic and $\mathrm{BD}+47^{\circ} 4206$ ) are well-known young emission-line stars. Our choice of stars is restricted by the availability of low-resolution UV spectra (both IUE SW and LW). A second restriction was made based on the quality of the observed spectra, which eliminates the following 6 stars from Table 1: WW Vul, BD $+24^{\circ} 676$, V350 Ori, R CrA, RR Tau, and RY Tau. The main conclusion from our analysis is that the BL contribution to the spectra in our sample can be simulated by spectra of type B5 I-A0 I, most frequently B9 Iab. In several cases the BL component in the UV spectrum is so strong that it influences the photometric bands in the near-UV and, therefore, the spectral classification based on photometry (e.g. NX Pup, BD+46 3471 , HK Ori, V380 Ori, and KK Oph). We also calculated the SEDs of the program stars in the visual and NIR. Some of these stars - NX Pup, V380 Ori, HK Ori, and HD 139614 - are observed at low inclinations (almost pole-on), which implies that their BL may also contribute significantly to the $U B V$-fluxes. The presence of this BL contribution may be an important reason why spectral classifications of the stars based on different distinct features of the spectrum have led to different spectral types.

In Sect. 6 we use the UV3C-model parameters to determine the position of the PMS stars in the HRD and to look for correlations between the evolutionary status of the star-disc-system, and its mass and c.s. extinction. And finally in Appendix A we discuss alternative interpretations of the spectral features of PMS Ae/Fe stars in the UV and compare our results with those of a few theoretical models.

\section{The UV3C-model}

\subsection{General description}

Our way of analysing the low-resolution UV spectra of PMS $\mathrm{Ae} / \mathrm{Fe}$ stars is similar to that developed by Bertout et al. (1988; BBB88 hereafter) for modeling the spectra and SEDs of T Tauri stars from the UV to the NIR. Arguments for the applicability of the model are given and fully discussed in that paper. We only summarise the main points. The UV spectra are analysed in terms of 3 spectral components: a contribution from the stellar photosphere; a contribution from an extended, geometrically thin, optically-thick, dusty accretion disc; and a contribution from the hot BL between the disc and photosphere. We show that with the parameters determined from IUE spectral analyses we obtain SEDs that reasonably agree with the photometric data in the visual and NIR, provided that the photometric $V$-magnitude is close to the $V$-magnitude during the IUE observation. Eventually, the remaining deviations in the IR regions can be partly removed by using more sophisticated disc models, but this does not affect the BL parameters, which are determined from the spectrum shortward of $1800 \AA$ where the BL component dominates.

\subsection{The photospheric component}

The photospheric component gives the spectral distribution of the stellar photosphere for a given set of parameters $\left(T_{\mathrm{eff}}\right.$, ${ }^{10} \log g$ and ${ }^{10} \log Z$ ). We simulate this component with a dereddened low-resolution UV spectrum from the IUE spectral atlases (Appendix G) because these well-classified spectra have the same resolution as the observed spectra of the PMS stars.
Comparison with spectra from computed model atmospheres (e.g. Kurucz models, Kurucz 1979, 1991) would be more elaborate and less accurate (because these models lack peculiar absorption lines that may still be unexplained). After we know the spectral type (ST) and luminosity class (LC) of the photosphere from the UV classification, we use Kurucz model fluxes to calculate the photospheric contributions in the visual and NIR photometric bands.

\subsection{The accretion disc}

Following BBB88 we assume that the disc is a flat, opticallythick, region with a continuum spectrum that can be simulated by the summation of a sequence of concentric, isothermal rings with Planck spectra. The local temperature $T_{\mathrm{d}}(r)$ of each ring can be derived from the sum of the local rate of viscous heating from accretion $F_{\mathrm{v}}(r)$ and the local rate of absorption of radiation from the stellar photosphere $F_{\mathrm{a}}(r)$ :

$\sigma T_{d}^{4}(r)=F_{\mathrm{v}}(r)+F_{\mathrm{a}}\left(r, T_{\star}\right)$.

The absorption from the stellar photosphere $F_{\mathrm{a}}\left(r, T_{\star}\right)$ has been evaluated by Adams \& Shu (1986):

$F_{\mathrm{a}}\left(r, T_{\star}\right)=\sigma \frac{T_{\star}^{4}}{2 \pi}(\varphi-\sin \varphi) \quad$ with $\quad \sin \varphi=\frac{R_{\star}}{r}$.

If in addition the disc is in Kepler rotation and the star rotates slowly, then

$F_{\mathrm{v}}(r)=\frac{3 G M_{\star} \dot{M}}{8 \pi r^{3}}(1-\sqrt{y}) \quad$ with $\quad y=\frac{R_{\star}}{r}$.

In this case the integrated dissipated energy in the disc is half of the total accretion luminosity $G M_{\star} R_{\star} \dot{M}$, where $M_{\star}$ and $R_{\star}$ are the stellar mass and radius and $\dot{M}$ is the accretion rate through the disc (Adams et al. 1987). The other half of this luminosity is dissipated in the BL, which we assume to be radiating as an optically-thick ring with radial thickness $\delta$ (see Sect. 2.4). We then can write:

$F_{\mathrm{v}}(r)=3 y^{3} \sigma \delta_{\mathrm{bl}} T_{\mathrm{bl}}^{4}(1-\sqrt{y}) \quad$ with $\quad \delta_{\mathrm{bl}}=\frac{\delta}{R_{\star}}$.

The flux of each disc ring can now be calculated from Eq. (1) and the disc contribution is evaluated by integrating over $r$ from $(1+\delta)$ to $R_{\text {disc }}$.

\subsection{The boundary-layer component}

Half of the total accretion luminosity $G M_{\star} R_{\star} \dot{M}$ is radiated away by the disc. The other half of this accretion luminosity is assumed to be spent in heating the BL, and with BBB88, we assume that the BL is radiating as an annular, optically-thick layer around the star with radial thickness $\delta$ :

$\frac{1}{2} G M_{\star} \dot{M}=4 \pi R_{\star}^{2} \sigma \delta T_{\mathrm{bl}}^{4}$

From an estimate of $T_{\mathrm{bl}}$ (from spectral shape in $\mathrm{SW}$ ) and $\delta_{\mathrm{bl}}$ (from the SW flux), we then can estimate the accretion rate $\dot{M}$ from the stellar mass $M_{\star}$ and radius $R_{\star}$. For fast rotating stars see Eq. (14) (Sect. 6). 


\section{The construction of the spectrum}

\subsection{Normalisation of the intrinsic (UV3C) spectrum}

The intrinsic spectra of photosphere and BL of the observed star for given spectral types and luminosity classes of the components were obtained from the standard spectra with the corresponding spectral and luminosity classes in the IUE spectral atlases of Heck \& Wu (Table G.6) by dividing their archived spectral fluxes by the ratio of their de-reddened flux $F^{0}(\lambda)$ in the Johnson $V$-band ( $V^{\mathrm{J}}$ hereafter) and the flux at $5500 \AA$ of the Kurucz model $\left(T_{\text {eff }}, g\right)$ for solar abundance and corresponding ST and LC:

$F^{0}(\lambda)=F(\lambda) 10^{C(\lambda)} \frac{\boldsymbol{F}\left(V_{\mathrm{kur}}^{5500}(T, g)\right)}{\boldsymbol{F}\left(V_{\mathrm{obs}}^{\text {iue }}-A_{\mathrm{v}}^{\text {is }}\right)}$

with $\boldsymbol{F}(V):=10^{-(8.446+0.4 V)}$

and $C(\lambda)=\frac{A_{\mathrm{v}}^{\text {is }}}{2.5}\left(\frac{A(\lambda)}{A\left(V^{\mathrm{J}}\right)}\right)_{R=3.1}$,

where $V_{\text {obs }}^{\text {iue }}$ is the observed $V$-band magnitude of the standard star with corresponding flux $\boldsymbol{F}(V), C(\lambda)$ gives the correction for the foreground extinction of the standard star, and $A_{\mathrm{v}}^{\text {is }}$ is the interstellar extinction in $V^{\mathrm{J}}$ :

$A_{\mathrm{v}}^{\text {is }}=R_{\mathrm{v}}^{\text {is }} E\left(B^{\mathrm{J}}-V^{\mathrm{J}}\right)^{\text {is }}:=R_{\mathrm{v}}^{\text {is }} \boldsymbol{E}^{\text {is }}$.

Equation (7) was derived from the flux calibration of the Johnson photometric system by Heber et al. (1984).

The intrinsic spectrum of the accretion disc for given $T_{\star}$, $T_{\mathrm{bl}}$, and $\delta_{\mathrm{bl}}$ was calculated from Eqs. (1)-(4), using the approximations of BBB88. The disc contribution is integrated outward up to $3.5 R_{\star}$, which has proved to be sufficient for the disccontribution in the UV. The disc inclination angle $i$ enters into the contributions of the 3 components; in the photospheric component it is due to shadowing by the disc; in the BL and disc components it results from screening by the star.

Figure 1 illustrates the 3 intrinsic UV spectral components for the case of NX Pup, together with their sum and the observed UV spectrum. The corresponding temperature distribution in the disc is given in Fig. 2. In order to compare this intrinsic sum spectrum with the observed UV spectrum, we have to normalise the intrinsic flux at a chosen wavelength (e.g. $5500 \AA$ ) and to deredden the intrinsic spectrum with interstellar extinction, which should give it the observed spectral shape.

If for values of the disc inclination angle $(i)$ (Sect. B.5) and BL width $\left(\delta_{\mathrm{bl}}\right)$ (BBB88 Eqs. (5), (9), (11)) we indicate the intrinsic (Kurucz model) $V^{\mathrm{J}}$ fluxes of the star, BL and disc by $F_{\mathrm{v}, \mathrm{s}}^{0}(i), F_{\mathrm{v}, \mathrm{b}}^{0}\left(i, \delta_{\mathrm{bl}}\right)$, and $F_{\mathrm{v}, \mathrm{d}}^{0}(i)$, respectively, we can normalise the sum of these fluxes to the "intrinsic observed flux of the system": the de-reddened observed flux $\left(F_{\mathrm{v}}^{\mathrm{d}}\right)$ in $V^{\mathrm{J}}\left(V_{\text {obs }}^{\text {iue }}\right)$ after additional correction for the (yet unknown) circumstellar absorption $A_{\mathrm{v}}^{\mathrm{cs}}$ in $V^{\mathrm{J}}$ :

$$
\begin{aligned}
F_{\mathrm{v}, \mathrm{t}}^{0}(i) & =F_{\mathrm{v}, \mathrm{s}}^{0}(i)+F_{\mathrm{v}, \mathrm{b}}^{0}\left(i, \delta_{\mathrm{bl}}\right)+F_{\mathrm{v}, \mathrm{d}}^{0}(i) \\
& =\left(\frac{d}{R_{\mathrm{s}}}\right)^{2} F_{\mathrm{v}}^{\mathrm{d}} 10^{0.4 A_{\mathrm{v}}^{\mathrm{cs}}(i)}
\end{aligned}
$$

with $F_{\mathrm{v}}^{\mathrm{d}}=\boldsymbol{F}\left(V_{\text {obs }}^{\text {iue }}-A_{\mathrm{v}}^{\text {is }}\right) \quad:=\boldsymbol{F}\left(V_{\text {der }}^{\text {iue }}\right)$ and $A_{\mathrm{v}}^{\mathrm{cs}}=R_{\mathrm{v}}^{\mathrm{cs}} E\left(B^{\mathrm{J}}-V^{\mathrm{J}}\right)^{\mathrm{cs}}:=R_{\mathrm{v}}^{\mathrm{cs}} E^{\mathrm{cs}}$,

where $R_{\mathrm{S}}$ is an (yet unknown) effective radius of the system (star+BL+disc) and $d$ the distance to the system. The stellar

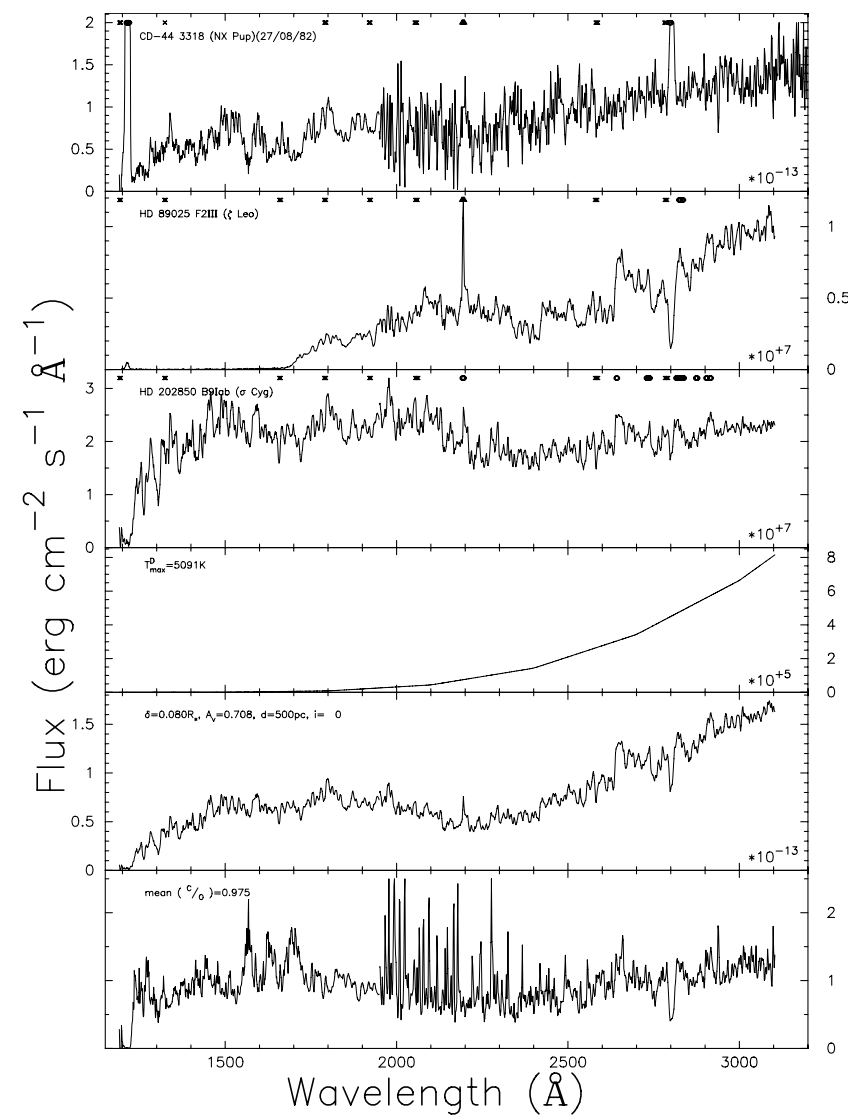

Fig. 1. From top to bottom: the observed UV spectrum of NX Pup (26-01), the 3 intrinsic components used in the UV3C model: F2 III photosphere $(\mathrm{HD} 89025)+\mathrm{B} 9 \mathrm{Iab} \mathrm{BL}(\sigma \mathrm{Cyg})+$ disc, the sum spectrum and the ratio of Calculated/Observed flux. For each component the flux normalisation factor is shown in the lower right corner (power of ten). See Table 2 for the model parameters and also Figs. 2 and 93.

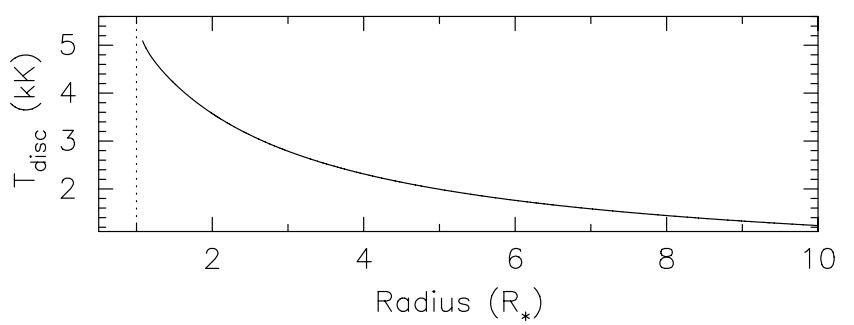

Fig. 2. Temperature distribution of the disc in the UV3C model (Eq. (1)) for NX Pup as used in Figs. 1 and 93.

(photospheric) radius $\left(R_{\star}\right)$ can be calculated from the ratio of the expected stellar flux at earth $\boldsymbol{F}\left(V_{\mathrm{s}}^{\mathrm{J}, 0}\right)$ with

$V_{\mathrm{s}}^{\mathrm{J}, 0}=5 \log d+M_{\mathrm{v}}-5$,

in which the absolute magnitude $M_{\mathrm{v}}$ for the star is taken from SK82 for the chosen ST and LC, and the intrinsic stellar flux $F_{\mathrm{v}, \mathrm{s}}^{0}(i)($ Eq. (9))

$\mathfrak{R}=\left(\frac{R_{\star}}{d}\right)_{\mathrm{s}}^{2}:=\frac{\boldsymbol{F}\left(V_{\mathrm{s}}^{\mathrm{J}, 0}\right)}{F_{\mathrm{v}, \mathrm{s}}^{0}(i)}$.

The circumstellar extinction $A_{\mathrm{v}}^{\mathrm{cs}}$ can be determined from

$A_{\mathrm{v}}^{\mathrm{cs}}(i)=V_{\mathrm{s}}^{\mathrm{J}, \mathrm{d}}(i)-V_{\mathrm{s}}^{\mathrm{J}, 0}$

with $\boldsymbol{F}\left(V_{\mathrm{s}}^{\mathrm{J}, \mathrm{d}}(i)\right)=\left(\frac{F_{\mathrm{v}, \mathrm{s}}^{0}(i)}{F_{\mathrm{v}, \mathrm{t}}^{0}(i)}\right) F_{\mathrm{v}}^{\mathrm{d}}$ and $V_{\mathrm{s}}^{\mathrm{J}, 0}$ from Eq. (10). 


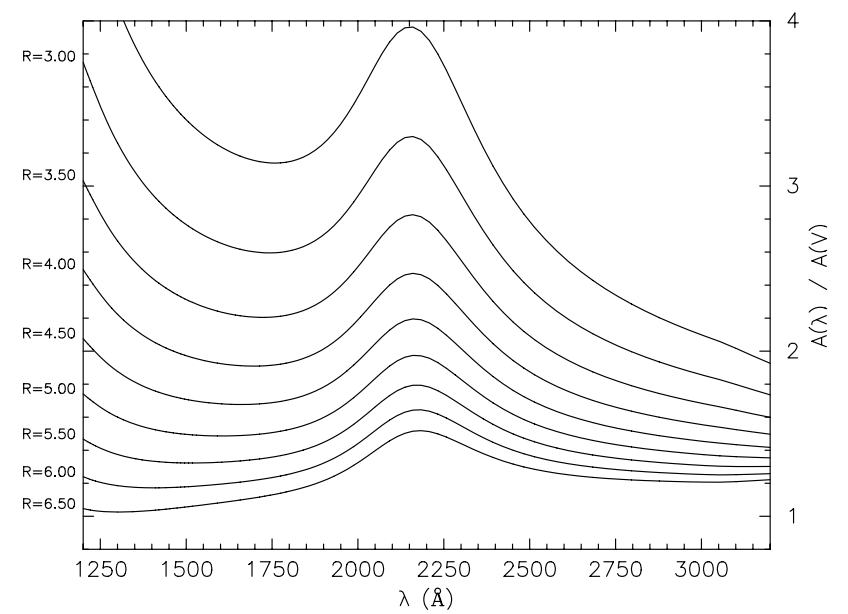

Fig. 3. Extinction curves in the UV (Cardelli et al. 1988, 1989).

In order to compare the calculated UV3C-model spectrum with the de-reddened observed spectrum, we need a circumstellar extinction law $A_{\lambda}^{\mathrm{cs}} / A_{\mathrm{v}}^{\mathrm{cs}}$ and the normalisation factor in Eq. (9):

$\boldsymbol{\aleph}=\left(\frac{R_{\mathrm{s}}}{d}\right)^{2}:=\frac{\boldsymbol{F}\left(V_{\mathrm{der}}^{\text {iue }}-A_{\mathrm{v}}^{\mathrm{cs}}\right)}{F_{\mathrm{v}, \mathrm{t}}^{0}(i)}$.

\subsection{Circumstellar extinction}

Since little is known about the extinction properties of the circumstellar dust around PMS Ae/Fe stars, we have assumed that the circumstellar extinction relation can be approximated by the same analytical expression as obtained by Cardelli et al. $(1988,1989)$ for the optical and UV extinction of the interstellar medium in various directions and parametrized by Eq. (8) for values of $\left[2.5 \leq R^{\text {is }} \leq 6.5\right]$. For $R^{\mathrm{cs}}$ we also use this range ${ }^{1}$.

The corresponding extinction curves are shown in Fig. 3. These extinction laws were not designed for circumstellar dust, but they generally appear to give satisfactory shapes of the UV3C-model spectra, although a good comparison of the simulated spectrum with the observed spectrum between 1800 and $2300 \AA$ (where $A_{\lambda} / A_{\mathrm{v}}$ reaches a maximum) is difficult, because of the high noise level in most of the observed spectra due to the low sensitivity of the IUE-LW cameras in that interval.

The method of parametrization with circumstellar dust parameters seems supported by how the large irregular brightness variations for HR 5999 and for the class of the UXORs ${ }^{2}$ correspond to variations in their UV spectra that can be approximated mainly by variations in $\boldsymbol{E}^{\mathrm{cs}}$ and $R^{\mathrm{cs}}$ (Tables $3-5$ ). Deviations in the spectra, which occur for high values of $R^{\mathrm{cs}}$, could be due to the changes in size or orientation of non-spherical circumstellar dust grains (Sects. 5.2 and 5.6).

\section{The IUE spectral analysis}

In order to match an UV3C model to an observed UV stellar spectrum and the flux in the photometric $V^{\mathrm{J}}$-band during the IUE spectral observation, we may vary 6 parameters: (1) the ST and

\footnotetext{
${ }^{1}$ Except for $\mathrm{BD}+09^{\circ} 880$ and HD 100546, which needed higher values to match the calculated spectrum properly to the observed spectrum, since $M_{\mathrm{v}}$ and $d$ are well-defined and leave no freedom to the other model parameters.

2 After Herbst (1994) and Eaton \& Herbst (1995), see also Sects. C.3, C.7, C.16, and C.25.
}

LC of the photosphere, (2) the ST and LC of the BL, (3) the stellar distance $d$, (4) the width $\delta$ of the BL, (5) the disc inclination angle $i$, and

(6) $R^{\mathrm{cs}}=A_{\mathrm{v}}^{\mathrm{cs}} / \boldsymbol{E}^{\mathrm{cs}}$, which specifies the extinction law for the circumstellar dust. With such a number of parameters we cannot claim to determine these parameters uniquely from the observed spectrum. However, the various parameters fortunately have different effects on specific wavelength regions in the calculated spectrum. We show these effects here for the case of NX Pup:

(a) For Ae and Fe stars, the wavelength interval where the continuum drops at shorter wavelength is especially sensitive to the ST and LC of the photosphere of the star (Fig. 4). In general the spectral type can be determined within 2 subtypes by comparing the observed UV spectrum (including the absorption features) with those of the well-classified standard stars in the IUE spectral atlases. The LC leads to a range of $M_{\mathrm{V}}$-values (especially for LC IV, III, II, and I) that we take from SK82.

(b) This range in $M_{\mathrm{v}}$ is further constrained by $d$ and the requirement that $A_{\mathrm{v}}^{\mathrm{cs}}$ should not be negative. Figure 5 shows calculated spectra for 4 different distances and $M_{\mathrm{v}}$-values, all of which give comparable agreement to the SW-part of the observed spectrum of NX Pup. For the two smaller distances the required $M_{\mathrm{V}}$-value was closer to that of an F2 IV star so we used the F2 V star from the list, causing the calculated spectrum to deviate more from the observed spectrum at longer wavelengths. Since NX Pup is probably associated with the Gum nebula; the distance is constrained to values around $500 \mathrm{pc}$, so that $M_{\mathrm{v}}$ must be close to $+1^{\mathrm{m}} 60$.

(c) In general the shape of the spectrum shortward of $\sim 1800 \AA$ is dominated by the spectral (late-B) type and LC ( I or II) of the BL, together with its width $\delta_{\mathrm{bl}}$. The spectral type and LC of the BL can be chosen from the IUE spectral atlases and estimated with an uncertainty of one subtype. In several cases (HD 163296, NX Pup, BD+46 3471, Z CMa, V380 Ori, and KK Oph), the flux at $\lambda<1800 \AA$ is receiving a considerable contribution from a BL; see the difference between the solid and dotted lines in Fig. 6, where the dotted lines give the calculated fluxes without the BL contribution. To some extent a hotter BL requires a smaller $\delta_{\mathrm{bl}}$ to obtain the same flux in this spectral range. Figure 6 shows the model calculations with 4 different BL-types and sizes applied to the spectrum of NX Pup, and illustrates our preference for a B9 Iab -type BL (e.g. $\sigma$ Cyg). In addition we find comparable matches to the spectrum of SU Aur with a B9 Iab type (Fig. 19) and a F0 Ib type BL (Fig. 20).

(d) The disc flux only contributes significantly to the total flux at $\lambda>2900 \AA$. However, due to its shadowing effect on the star and the BL, the disc inclination angle $i$ affects the slope of the calculated spectrum, especially for $\lambda<2300 \AA$ (Fig. 7).

(e) Figure 8 shows the effect of varying $R^{\text {cs }}$ from 2.5 to 6.5 for $M_{\mathrm{v}}=1 \mathrm{~m} .70$, which is mainly shortward of $2200 \AA$.

After an initial choice of the parameter set based on information from the visual spectral regions (Appendices B and C), we made a progressive match to the observed UV spectrum by varying the parameters in a certain order. First we made a rough match by varying the ST and LC (in particular $M_{\mathrm{v}}$ ) of the photosphere, the BL, and width $\delta_{\mathrm{bl}}$. Then the distance of the star could be varied within the margins of its uncertainty, implying a corresponding variation of $A_{\mathrm{v}}^{\mathrm{cs}}$, which should be limited to positive values. We then varied the inclination angle $i$ of the disc and the extinction law $R^{\mathrm{cs}}$ in order to improve the agreement in the 
Table 1. Observational data of the PMS stars. The spectral classifications based on different criteria are separated by $(/)$ to distinguish them from the given spectral ranges (-). Additional information on the double stars (denoted by $\ddagger$ in Col. 2) is given in Table B.1. Uncertain values are indicated with (:). The foreground colour-excess $\boldsymbol{E}^{\text {is }}$ is labelled with $\left(^{*}\right)$ if $R^{\text {is }} \neq 3.1$ (Appendix B.4). The $h$ in Col. 7 denotes Hipparcos values (ESA 1997), see Appendix C for supplementary information on each star. Column 9 indicates if Walraven photometry was obtained for the star. The inclination is indicated with E or P based on the methods (X, Y, Z) described in Appendices B.5 and D.

\begin{tabular}{|c|c|c|c|c|c|c|c|c|c|c|c|}
\hline \multirow{3}{*}{$\begin{array}{r}\overline{(1)} \\
\#\end{array}$} & \multirow{3}{*}{\multicolumn{2}{|c|}{$\begin{array}{c}(2) \\
\text { PMS star }\end{array}$}} & (3) & (4) & (5) & (6) & \multirow{3}{*}{$\begin{array}{c}(7) \\
d \\
(\mathrm{pc}) \\
\end{array}$} & \multirow{3}{*}{$\begin{array}{c}(8) \\
v \sin i \\
\left(\mathrm{~km} \mathrm{~s}^{-1}\right)\end{array}$} & \multirow{3}{*}{$\begin{array}{l}\text { (9) } \\
\mathrm{w}\end{array}$} & \multicolumn{2}{|c|}{ (10) } \\
\hline & & & $V_{\text {phot }}$ & $\boldsymbol{E}^{\text {is }}$ & \multicolumn{2}{|c|}{ Spectral classification } & & & & $i$ & \\
\hline & & & \multicolumn{2}{|c|}{ (magnitude) } & TCD & visual spectra & & & & & $\mathrm{X} \mathrm{Y} \mathrm{Z}$ \\
\hline 1 & XY Per & † HD 275877 & $9-10.5$ & 0.19 & A1-A3 & A2IIe & $350^{15}$ & $130:+95:^{13}, 217^{44}$ & - & & r: 12 IIB-R ${ }^{25}$ \\
\hline 2 & WW Vul & HD 344361 & $10.4-12.6$ & 0.3: & A2-A5 & $\mathrm{A} 0-3 \mathrm{Ve}$ & $534^{16}$ & $125^{40}, 220^{44}, 150^{45}$ & + & $\mathrm{E}$ & r $12 \mathrm{IIBR}^{26}$ \\
\hline 3 & UX Ori & $\ddagger$ HD 293782 & $8.7-12.8$ & 0.06 & $\mathrm{~A} 2-\mathrm{A} 3$ & A3IIIe & 400 & $70^{1}, 215^{44}, 140^{45}$ & + & $\mathrm{E}$ & $\mathrm{r} 18 \mathrm{IIIRm}^{26}$ \\
\hline 4 & HD 163296 & MWC 275 & $6.79-7.01$ & 0.00 & B9-A0 & $\mathrm{A} 0 / \mathrm{A} 7 \mathrm{Ve}$ & $122^{h}$ & $120^{2,3}, 133^{44}$ & + & $\mathrm{E}$ & n 24 III-IIB ${ }^{17}$ \\
\hline 5 & HD 150193 & † MWC 863 & 8.83 & 0.03 & A3-A5 & $\mathrm{A} 4 \mathrm{Ve}$ & $150^{h}$ & $100^{2}$ & + & $\mathrm{E}$ & $\mathrm{n} 21 \mathrm{IVB}^{17}$ \\
\hline 6 & $\mathrm{BD}+24^{\circ} 676$ & $\ddagger$ HD 283817 & $10.30-10.56$ & 0.04 & F-G & $\mathrm{A} 3 \mathrm{e}$ & $150^{41}$ & - & + & $\mathrm{E}$ & $\mathrm{o}: 17 \mathrm{IIB}^{20}$ \\
\hline 7 & BF Ori & $\neq \mathrm{BD}-06^{\circ} 1259$ & $9.7-13.5$ & 0.06 & A3-A5 & A5II-IIIe & 450 & $100^{1}, 80^{3}, 37^{44}, 40^{45}$ & + & $\mathrm{E}$ & r $20 \mathrm{IIIRm}^{26} \mathrm{IIB}^{17}$ \\
\hline 8 & HD 31648 & MWC 480 & 7.7 & 0.02 & A5-A6 & $\mathrm{A} 2-3 \mathrm{Ve}$ & $131^{h}$ & $80^{34}, 102^{44}$ & + & $\mathrm{E}$ & n 24 III $^{19}$ \\
\hline 9 & HR 5999 & $\ddagger$ V856 Sco & $6.72-8.2$ & 0.03 & A5-A7 & A5-7eIII-V & $210^{h}$ & $180^{14}$ & + & $\mathrm{E}$ & r 17 IIB $^{14,21,26}$ \\
\hline 10 & $\beta \mathrm{Pic}$ & HD 39060 & 3.85 & 0.00 & A5-A7 & A5V-IV & $19.3^{h}$ & $120^{4}$ & - & $\mathrm{E}$ & n $12-$ \\
\hline 11 & HD 104237 & DX Cha & 6.60 & 0.02 & A5-A7 & $\mathrm{A} 4 \mathrm{Ve}$ & $116^{h}$ & $150^{5}$ & + & $\mathrm{E}$ & n 26 III-IVB $^{19}$ \\
\hline 12 & HD 169142 & †: MWC 925 & 8.11 & 0.00 & B9 & $\mathrm{A} 5 \mathrm{Ve}$ & $145^{30}$ & $55^{31}$ & - & $\mathrm{P}$ & n $7 \mathrm{I}^{32}$ \\
\hline 13 & HD 36112 & MWC 758 & 8.26 & 0.06 & A5-A7 & A3e: & $200^{h}$ & $60^{33}$ & - & $\mathrm{E}$ & n 20: $\operatorname{IVB}^{22}$ \\
\hline 14 & HD 139614 & $\ddagger: C D-42^{\circ} 10650$ & 8.26 & 0.02 & A7 & $\mathrm{A} 7 \mathrm{Ve}$ & $157^{30}$ & $13^{29}, 24^{31}$ & - & $\mathrm{P}$ & n $\quad 5 \mathrm{I}^{32}$ \\
\hline 15 & HD 142666 & V1026 Sco & $8.65-8.84$ & 0.22 & $\mathrm{~F} 2 \mathrm{~V}$ & $\mathrm{~A} 3 / \mathrm{A} 8 \mathrm{Ve}$ & $114^{30}$ & $56^{29}, 70^{31}, 72^{44}$ & - & $\mathrm{E}$ & $\mathrm{r}: 17 \mathrm{IIR}^{32}$ \\
\hline 16 & V346 Ori & HD 287841 & $10.0-11.9$ & 0.06 & $\mathrm{~A} 3-\mathrm{A} 4$ & A5IIIe & 450 & - & + & $\mathrm{E}$ & $\mathrm{r} \quad 16 \mathrm{I}^{19}$ \\
\hline 17 & V350 Ori & - & $10.3-13.2$ & 0.06 & A4-F0 & A0-A1IVe & 450 & - & + & $\mathrm{E}$ & r 17 IIBR $^{13,19}$ \\
\hline 18 & V351 Ori & HD 38238 & $8.3-11.6$ & 0.06 & A3-A7 & A7IIIe & 450 & - & + & $\mathrm{E}$ & $\mathrm{r} \dagger 20 \mathrm{IIIR}^{19}$ \\
\hline 19 & BN Ori & HD 245465 & 9.65 & 0.07 & A5-F2 & A7-G9III-IVe & 450 & $220^{6}$ & + & $\mathrm{E}$ & $\mathrm{r} \dagger 17 \mathrm{IIBR}^{6,25}$ \\
\hline 20 & HD 35929 & $\mathrm{BD}-08^{\circ} 1128$ & 8.13 & 0.06 & A5-A7 & A8III: & 450 & $150:^{12}$ & - & $\mathrm{E}$ & r: $15 \mathrm{I}^{19}$ \\
\hline 21 & HD 144432 & $\mathrm{CD}-27^{\circ} 10778$ & 8.15 & 0.00 & A7V & $\mathrm{A} 9 \mathrm{Ve}$ & $200^{h}$ & $54^{29}, 74^{31}, 85^{44}$ & - & $\mathrm{E}$ & $\mathrm{n}: 15$ III-IVB ${ }^{27,32}$ \\
\hline 22 & $\mathrm{BD}+47^{\circ} 4206$ & $6 \quad$ SAO53184 & 9.30 & 0.05 & - & A9III & $260^{h}$ & - & - & - & n $13-$ \\
\hline 23 & FU Ori & $\mathrm{BD}+09^{\circ} 5427$ & 9.3 & 0.06 & G0-G2 & F2/GI-IIe/K0 & 450 & $60^{8}$ & + & $\mathrm{E}$ & $\mathrm{r} \dagger 40 \mathrm{IVB}^{26}$ \\
\hline 24 & $\mathrm{R} \mathrm{CrA}$ & $\mathrm{CD}-37^{\circ} 13027$ & $10.0-14.4$ & $0.18 *$ & F2pe & A5-F5pe & $140^{h:}$ & - & + & $\mathrm{E}$ & r 14 IIRB $^{26,28}$ \\
\hline 25 & CQ Tau & HD 36910 & $9.9-11.6$ & 0.07 & A 8 & A8-F2IIIe & $100^{h}$ & $110^{1}, 105^{44}, 90^{45}$ & + & $\mathrm{E}$ & r 40 III-IVR ${ }^{23}$ \\
\hline 26 & NX Pup & $\neq C D-44^{\circ} 3318$ & $9.8-11.2$ & 0.00 & F0-F4 & B9-F0-2IIIe & 500 & $120^{1}$ & + & $\mathrm{P}$ & o $12 \mathrm{IIB}^{26}$ \\
\hline 27 & $\mathrm{BD}+46^{\circ} 3471$ & $1+$ V1578 Cyg & $10.1-10.2$ & 0.31 & A5 & A2-A4IIIe & $900^{39}$ & $150^{1}, 100^{3}$ & - & $\mathrm{E}$ & -19 IVB $^{17}$ \\
\hline 28 & HK Ori & $\div$ MWC 497 & 11.6 & 0.06 & F5 & $\mathrm{B} 8 / \mathrm{A} 4 \mathrm{e}$ & 450 & $175^{3}, 150^{40}$ & + & $\mathrm{P}$ & o $10{\mathrm{I}-\mathrm{IIB}^{26}}^{26}$ \\
\hline 29 & AK Sco & $\ddagger$ HD 152404 & $11.0-11.4$ & 0.13 & F5-F6 & F5IVe & $150^{h}$ & $19^{9}$ & + & $\mathrm{E}$ & r $20 \mathrm{IIR}^{26}$ \\
\hline 30 & $\mathrm{ZCMa}$ & $\ddagger$ HD 53179 & $8.8-11.2$ & $0.16^{*}$ & F-G & B5-8/F5-7Ie & $1050^{37}$ & $100^{38}$ & + & E & o $40 \mathrm{IVB}^{26}$ \\
\hline 31 & HD 199143 & $+\mathrm{BD}-17^{\circ} 6127$ & 7.1 & 0.0 & - & F8Ve & $48^{h}$ & $200:^{36}, 155^{44}$ & - & - & $-13 \mathrm{em}^{36}$ \\
\hline 32 & V380 Ori & $\ddagger \mathrm{BD}-06^{\circ} 1253$ & $10.0-11.5$ & 0.06 & $\mathrm{~F}$ & B8-A2e & 450 & $20^{3}, 200^{40}$ & + & $\mathrm{P}$ & o $22 \mathrm{I}^{26}$ \\
\hline 33 & NV Ori & $\mathrm{BD}-05^{\circ} 1324$ & $10.0-10.6$ & 0.06 & $\mathrm{~F} 0-\mathrm{F} 2 \mathrm{e}$ & F2-8III-IVe & 450 & $80^{1}, 81^{44}$ & + & $\mathrm{E}$ & $\mathrm{r} \quad 20 \mathrm{em}^{13}$ \\
\hline 34 & RR Tau & $\mathrm{BD}+26^{\circ} 887 a$ & $10.2-14.2$ & 0.07 & A4-F0 & B8-A2II-IIIe & $600^{24}$ & $>60^{3}, 225^{44}, 140^{45}$ & - & $\mathrm{E}$ & r $20 \mathrm{IIR}^{17}$ \\
\hline 35 & KK Oph & $\ddagger-$ & $9.4-12.8$ & 0.32 & A5 & $\mathrm{A} 5-7 \mathrm{Ve}$ & $<250^{35}$ & $85^{3,12}, 177^{44}$ & + & $\mathrm{E}$ & r $22 \mathrm{IIR}^{19}$ \\
\hline 36 & SU Aur & HD 282624 & $9.0-11.8$ & 0.12 & F5-G2 & G2IIIe/K: & $144^{h}$ & $66^{10}$ & - & $\mathrm{E}$ & r $40 \mathrm{IIB}^{25}$ \\
\hline 37 & RY Tau & $\ddagger$ HD 283571 & $10.42-11.12$ & 0.04 & F8-K1 & K1IV-Ve/K5: & $134^{h}$ & $55^{44}$ & - & $\mathrm{E}$ & r $35 \mathrm{IIB}^{18}$ \\
\hline 38 & HD 283572 & V987 Tau & 8.9-9.1 & 0.03 & G2-G5 & G5IVe & $128^{h}$ & $130^{11}, 80^{7}$ & - & $\mathrm{E}$ & o $40 \mathrm{abs}$ \\
\hline 39 & $\mathrm{BD}+09^{\circ} 880$ & HD 245185 & $9.83-9.9$ & 0.06 & B9 & B9/A5Ve & 450 & $80^{2}, 150^{40}$ & - & $\bar{P}$ & $n<5 \mathrm{I}^{25}$ \\
\hline 40 & HD 100546 & KR Mus & $6.67-6.77$ & 0.00 & A0 & B9Ve & $103^{h}$ & $250^{12}$ & + & $\mathrm{E}$ & $\mathrm{n}<5 \mathrm{IIR}^{12}$ \\
\hline 41 & HD 98922 & $\neq C D-52^{\circ} 4340$ & $6.7-6.8$ & 0.05 & A0 & B9Ve & $200^{42}$ & - & + & $\mathrm{E}$ & $-6 \operatorname{IVB}^{19}$ \\
\hline 42 & HD 97048 & CU Cha & $8.38-8.56$ & $0.06 *$ & B9-A0 & $\mathrm{B} 9.5-\mathrm{A} 0 \mathrm{e}$ & $180^{h}$ & $140^{1,2}$ & + & $\mathrm{E}$ & n 8 IVB $^{26}$ \\
\hline 43 & HD 95881 & $\mathrm{CD}-70^{\circ} 775$ & $8.2-8.4$ & 0.17 & - & A1IIIJ & $300^{43}$ & $150^{12}$ & - & $\mathrm{E}$ & $-11 \mathrm{IIB}^{19}$ \\
\hline
\end{tabular}

1: Böhm \& Catala (1995) 10: Bertout et al. (1988)

2: Finkenzeller (1985)

12: Grady et al. (1996)

4: Slettebak (1982)

5: Appendix C.11

6: Shevchenko et al. (1997)

7: Joncour et al. (1994)

8: Herbig (1966)

9: Andersen et al. (1989)
13: Herbig \& Bell (1988)

14: Tjin A Djie et al. (1989)

15: Herbertz et al. (1991)

16: Friedemann et al. (1993)

17: Finkenzeller \& Mundt (1984)

18: Kolotilov \& Zajtseva (1974)
19: de Winter et al. (2001) 28: Hamann \& Persson (1992) 37: Shevchenko et al. (1999) 20: Martin (1993) 29: Meeus et al. (1998)

21: Bessel \& Eggen (1972) 30: Sylvester et al. (1996)

22: Pogodin (1995) 31: Dunkin et al. (1997a)

23: Berdyugin et al. (1990) 32: Dunkin et al. (1997b)

24: Artyukhina (1960) 33: Beskrovnaya et al. (1999)

25: Fernández et al. (1995) 34: Glagolevskii et al. (1998)

26: Reipurth et al. (1996) 35: Wallenquist (1937)

27: Pérez et al. (2004) 36: v.d.Ancker et al. (2000)
38: Hartmann et al. (1989) 39: Elias (1978b)

40: Corporan \& Lagrange (1999)

41: Weaver \& Hobson (1988)

42: Appendix C. 41

43: Appendix C.43

44: Mora et al. (2001)

45: Grinin et al. (2001)
LW $(\lambda>2000 \AA)$ spectral range. After that $M_{\mathrm{v}}, \delta_{\mathrm{bl}}$, and $R^{\mathrm{cs}}$ were slightly adjusted until the match was optimal.

We note that it sometimes occurred that matches of comparable quality could be obtained for distinctly different spectral types. In such cases it was necessary to compensate for the difference in $M_{\mathrm{v}}$ by a change in $R^{\mathrm{cs}}$. Also for a change in distance (in cases where the distance is not well known), a change in $M_{\mathrm{v}}$ and $R^{\mathrm{cs}}$ sometimes produced equally good matches to the observed spectrum. Examples of such multiple solutions can be found in Table 2 and are discussed in the next section. 
Table 2. Data of the analysed stars (Part 1), (3) see Table 3, (5) see Table 5.

\begin{tabular}{|c|c|c|c|c|c|c|c|c|c|c|c|c|c|}
\hline \# & PMS star & $\begin{array}{c}\text { IUE } \\
\text { images }\end{array}$ & $\begin{array}{l}\text { Photo- } \\
\text { sphere }\end{array}$ & $\begin{array}{l}\text { Bound. } \\
\text { Layer }\end{array}$ & $\begin{array}{l}T_{\mathrm{d}}^{\max } \\
(\mathrm{K})\end{array}$ & 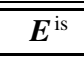 & $\frac{V_{\text {obs }}^{\text {iue }}}{(\mathrm{mag}}$ & $\frac{M_{\mathrm{v}}}{\text { itude) }}$ & 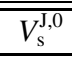 & $R^{\mathrm{cs}}$ & \multicolumn{2}{|c|}{$\begin{array}{cr}d & \delta_{\mathrm{bl}} \\
(\mathrm{pc}) & \left(10^{-3}\right)\end{array}$} & $\begin{array}{c}i \\
\left({ }^{\circ}\right)\end{array}$ \\
\hline 1 & XY Per & 01-01 & A2 III & B5 $\mathrm{Ib}^{1}$ & 7209 & 0.19 & 9.36 & +0.30 & 8.02 & 3.10 & 350 & 10 & 60 \\
\hline 3 & UX Ori & 03-01 & A2 III & B9 Iab & 6662 & 0.06 & 9.66 & +0.30 & 8.31 & 5.00 & 400 & 60 & 60 \\
\hline 3 & & 03-05 & A 2 III & B9 Iab & 6662 & 0.06 & 10.65 & +0.30 & 8.31 & 5.00 & 400 & 60 & 60 \\
\hline 3 & & 03-08 & A2 III & B9 Iab & 6662 & 0.06 & 10.86 & +0.30 & 8.31 & 5.50 & 400 & 60 & 60 \\
\hline $3 a$ & & $03-01$ & A3 III & B9 Iab & 6080 & 0.06 & 9.66 & +0.50 & 8.51 & 5.00 & 400 & 125 & 60 \\
\hline $3 a$ & & $03-05$ & A3 III & B9 Iab & 6080 & 0.06 & 10.65 & +0.50 & 8.51 & 5.50 & 400 & 125 & 60 \\
\hline $3 a$ & & 03-08 & A3 III & B9 Iab & 6080 & 0.06 & 10.86 & +0.50 & 8.51 & 6.00 & 400 & 125 & 60 \\
\hline 4 & HD 163296 & 04-01 & $\mathrm{A} 3 \mathrm{~V}^{1}$ & B9 Iab & 6339 & 0.00 & 6.90 & $+1.25^{4}$ & 6.68 & 5.00 & 122 & 80 & 57 \\
\hline 4 & & 04-01 & A3 III & B9 Iab & 6526 & 0.00 & 6.90 & $+1.25^{4}$ & 6.68 & 6.50 & 122 & 80 & 57 \\
\hline $4 b$ & & 04-01 & $+v$ & $9 \mathrm{Iab}$ & 6273 & 0.00 & 6.90 & $+1.25^{4}$ & 6.68 & 6.50 & 122 & 60 & 57 \\
\hline $4 \mathrm{~b}$ & & 04-01 & & $\mathrm{Ib}$ & 273 & 0.00 & 6.90 & $+1.85^{z}$ & 7.10 & 3.10 & 111 & 60 & 57 \\
\hline 5 & HD 150193 & 05-01 & A3 III & $9 \mathrm{Iab}$ & 532 & 0.03 & 8.83 & +0.30 & 6.18 & 5.15 & 150 & 20 & 67 \\
\hline 7 & BF Ori & (3) & A4 V & $9 \mathrm{Iab}$ & 6627 & 0.06 & (3) & $+1.25^{4}$ & 9.26 & (3) & 400 & 20 & 60 \\
\hline $7 \mathrm{a}$ & & (3) & A5 $\mathrm{V}^{2}$ & $9 \mathrm{Iab}$ & 6424 & 0.06 & (3) & $+1.30^{4}$ & 9.31 & (3) & 400 & 20 & 60 \\
\hline $7 b$ & & (3) & A5 $\mathrm{III}^{1}$ & 1 & 346 & 0.0 & (3) & +0.70 & 8.71 & (3) & 400 & 20 & 60 \\
\hline $7 \mathrm{c}$ & & (3) & A6 IV & 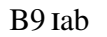 & 229 & 0.0 & (3) & +1.50 & 9.51 & (3) & 400 & & 60 \\
\hline $7 \mathrm{~d}$ & & (3) & A6 III & $9 \mathrm{Iab}$ & 6229 & 0.0 & (3) & +0.50 & 8.51 & (3) & 400 & & 60 \\
\hline $7 \mathrm{e}$ & & (3) & A7 IV & $9 \mathrm{Iab}$ & 6073 & 0.06 & (3) & +1.70 & 9.71 & (3) & 400 & 2 & 60 \\
\hline $7 \mathrm{f}$ & & (3) & A7 III & $9 \mathrm{Iab}$ & 5995 & 0.06 & (3) & +1.10 & 9.11 & (3) & 400 & 21 & 60 \\
\hline $7 p$ & & $07-02$ & $\mathrm{~A} 4 \mathrm{~V}$ & $9 \mathrm{Iab}$ & 6627 & 0.06 & 9.89 & +1.70 & 9.71 & 3.10 & 400 & 2 & 60 \\
\hline $7 \mathrm{p}$ & & $07-02$ & $\mathrm{~A} 4 \mathrm{~V}$ & $9 \mathrm{Iab}$ & 6417 & 0.06 & 9.89 & +1.70 & 9.71 & 3.10 & 400 & 40 & 60 \\
\hline 8 & HD 31648 & 08-01 & $\mathrm{A} 5 \mathrm{~V}^{2}$ & B9 Iab & 6534 & 0.02 & 7.71 & $+2.10^{z}$ & 7.69 & 6.50 & 131 & 12 & 38 \\
\hline 8 & & 08-02 & $\mathrm{A} 5 \mathrm{~V}^{2}$ & B9 Iab & 6534 & 0.02 & 7.71 & $+2.10^{z}$ & 7.69 & 3.10 & 131 & 12 & 38 \\
\hline 8 & & $08-03$ & $\mathrm{~A} 5 \mathrm{v}^{2}$ & B9 Iab & 6602 & 0.02 & 7.71 & $+2.10^{z}$ & 7.69 & 3.10 & 131 & 8.0 & 38 \\
\hline 9 & HR 5 & 09-01 & A5 $\mathrm{III}^{2}$ & B9 Iab & 6584 & 0.00 & 6.72 & -0.35 & 6.26 & (5) & 210 & 5.0 & 53 \\
\hline $9 a$ & & 09-01 & A6 IV & $9 \mathrm{Iab}$ & 6462 & 0.00 & 6.72 & $-0.35^{3}$ & 6.26 & (5) & 210 & 5.0 & 53 \\
\hline $9 b$ & & 09-01 & A7 III & B9 & 6219 & 0.00 & 6.72 & -0.25 & 6.36 & (5) & 210 & 5.0 & 53 \\
\hline 10 & $\beta$ Pic & $10-01$ & A5 $\mathrm{v}^{2}$ & - & 885 & 0.00 & 3.85 & $+2.10^{z}$ & 3.53 & 3.10 & 22.3 & 0 & 90 \\
\hline $10 \mathrm{a}$ & & $10-01$ & A7 V & - & 6591 & 0.00 & 3.85 & $+2.34^{z}$ & 3.83 & 3.10 & 19.3 & U & 90 \\
\hline 11 & HD 104237 & $11-01$ & A7 IV & B9 Iab & 6259 & 0.02 & 6.60 & +1.30 & 6.62 & 3.10 & 116 & 7.0 & 53 \\
\hline 11 & & $11-02$ & A7 IV & B9 Jah & 6300 & 0.02 & 6.60 & +1.30 & 6.62 & 3.10 & 116 & 5.0 & 53 \\
\hline 11 & & $11-03$ & A7 IV & B & 6278 & 0.02 & 6.60 & +1.30 & 6.62 & 3.10 & 116 & 6.0 & 53 \\
\hline 12 & HD 1 & $12-01$ & A7 V & $\mathrm{B}_{2}$ & 6464 & 0.00 & 8.10 & $+2.40^{z}$ & 8.29 & 3.10 & 151 & 2.0 & 0 \\
\hline 13 & $\mathrm{HD} 3$ & $13-01$ & III & $A$ & 6126 & $0 \Omega$ & 8.10 & +1.10 & 7.66 & 4.25 & 200 & 10 & 70 \\
\hline 14 & $\mathrm{H}$ & 01 & & & & & 8.26 & $+2.40^{z}$ & 8.38 & 3.10 & 157 & 1.0 & 0 \\
\hline 15 & & 01 & & $\mathrm{E}$ & 9 & 0. & 9.10 & +1.10 & 6.39 & 6.50 & 1 & 5. & 45 \\
\hline 16 & V346 Ori & $16-01$ & III & B & 6299 & 0.0 & 10.20 & +1.10 & 9.11 & 4.50 & 400 & 2 & 60 \\
\hline $16 \mathrm{a}$ & & $16-01$ & & & 6464 & 0.0 & 10.20 & +2.20 & 8.71 & 4.50 & 200 & 2 & 60 \\
\hline 18 & V351 Ori & $18-01$ & A7 III & $9 \mathrm{Iab}$ & 6219 & 0.0 & 8.92 & +0.10 & 8.37 & 3.10 & 450 & 5 & 45 \\
\hline $18 \mathrm{a}$ & & $18-02$ & A7 III & $9 \mathrm{I}$ & 6299 & 0.06 & 8.92 & +0.15 & 8.42 & 3.10 & 450 & 2. & 45 \\
\hline 19 & BN Ori & $19-01$ & F0 III & $9 \mathrm{I}$ & 5887 & 0.0 & 9.68 & +1.50 & 9.51 & 3.10 & 400 & 2. & 60 \\
\hline 19 & & $19-02$ & F0 III & B9 I & 5887 & 0.06 & 9.68 & +1.50 & 9.51 & 3.10 & 400 & $2 .(1$ & 60 \\
\hline 19 & & $19-03$ & F0 III & B9 Iab & 5887 & 0.06 & 9.68 & +1.50 & 9.51 & 3.10 & 400 & 2.0 & 60 \\
\hline 20 & HD 35929 & $20-01$ & F0 III & B9 Iab & 5872 & 0.06 & 8.10 & +1.50 & 7.72 & 3.10 & 175 & 3.0 & 80 \\
\hline 20 & & $20-02$ & F0 III & B9 Iab & 5872 & 0.06 & 8.10 & +1.50 & 7.72 & 3.10 & 175 & 3.0 & 80 \\
\hline $20 \mathrm{a}$ & & $20-02$ & F0 III & B9 Iab & 5887 & 0.06 & 8.10 & -0.30 & 7.71 & 3.10 & 400 & 2.0 & 80 \\
\hline $20 \mathrm{~b}$ & & $20-01$ & F0 V & B9 Iab & 5929 & 0.06 & 8.10 & +2.70 & 7.47 & 3.10 & 90 & 2.0 & 80 \\
\hline $20 \mathrm{~b}$ & & $20-02$ & $\mathrm{~F} 0 \mathrm{~V}$ & B9 Iab & 5929 & 0.06 & 8.10 & +2.70 & 7.47 & 3.10 & 90 & 2.0 & 80 \\
\hline 21 & HD 144432 & $21-01$ & A9 III & B9 Iab & 6011 & 0.00 & 8.20 & $+1.53^{4}$ & 8.24 & 3.10 & 220 & 2.0 & 80 \\
\hline 21 & & $21-02$ & A9 III & B9 Iab & 6011 & 0.00 & 8.20 & $+1.53^{4}$ & 8.24 & 3.10 & 220 & 2.0 & 80 \\
\hline $21 \mathrm{a}$ & & $21-01$ & A8 V & B9 Iab & 6241 & 0.00 & 8.20 & $+1.50^{4}$ & 7.89 & 3.10 & 190 & 2.0 & 80 \\
\hline $21 \mathrm{a}$ & & $21-02$ & $\mathrm{~A} 8 \mathrm{~V}$ & B9 Iab & 6241 & 0.00 & 8.20 & $+1.50^{4}$ & 7.89 & 3.10 & 190 & 2.0 & 80 \\
\hline 22 & $\mathrm{BD}+47^{\circ} 4206$ & $22-01$ & F0 III & B9 Iab & 5887 & 0.05 & 9.26 & +0.90 & 9.11 & 3.10 & 440 & 2.0 & 85 \\
\hline $22 \mathrm{a}$ & & $22-01$ & F0 III & B9 Iab & 5887 & 0.05 & 9.26 & +1.30 & 9.08 & 3.10 & 360 & 2.0 & 85 \\
\hline
\end{tabular}

\section{Results of the analysis}

The observational information on the stars analysed here is contained in Appendix B. Table 1 gives a summary of the most important parameters as obtained from visual observations. The selection of input data for the UV3C model analysis is discussed in Appendix C, and the final model parameters are listed in Tables $2-6^{3}$. In Figs. 10-17 and 45-110 the corresponding UV3C spectra are compared with the observed spectra. Fluxes at

3 The numbers in Col. 3 refer to the IUE images used for the observed spectrum (Appendix F). The spectral types in Cols. 4 and 5 refer to those used for the photosphere and boundary-layer (Appendix G). Labels ${ }^{1}$ and ${ }^{2}$ are used to distinguish comparison stars with the same ST. Column 7 gives the foreground colour excess used to de-redden 
Table 2. Data of the analysed stars (Part 1 continued), (3) see Table 3, (5) see Table 5.

\begin{tabular}{|c|c|c|c|c|c|c|c|c|c|c|c|c|}
\hline \multicolumn{3}{|c|}{$F_{\text {rel }}(5500 \AA) \times 100$} & \multirow{2}{*}{$\begin{array}{c}\boldsymbol{\kappa} \\
\left(10^{-20}\right)\end{array}$} & \multirow{2}{*}{$\begin{array}{c}A_{\mathrm{v}}^{\mathrm{cs}} \\
(\mathrm{mag})\end{array}$} & \multirow{2}{*}{$\begin{array}{c}R_{\star} \\
\left(R_{\odot}\right) \\
\end{array}$} & \multirow{2}{*}{$\begin{array}{l}L_{\star} \\
\left(L_{\odot}\right)\end{array}$} & \multirow{2}{*}{$\begin{array}{c}M_{\star} \\
\left(M_{\odot}\right) \\
\end{array}$} & \multirow{2}{*}{\multicolumn{2}{|c|}{$\begin{array}{cc}\dot{M} & \dot{M}^{\mathrm{c}} \\
\left(10^{-8} M_{\odot} \mathrm{yr}^{-1}\right)\end{array}$}} & \multirow{2}{*}{$\begin{array}{c}t_{\star} \\
(\mathrm{Myr})\end{array}$} & \multirow[b]{2}{*}{ \# } & \multirow[b]{2}{*}{ Fig. } \\
\hline star & $\mathrm{BL}$ & disc & & & & & & & & & & \\
\hline 85.8 & 1.4 & 12.8 & 5.96 & 0.93 & 3.29 & 63.3 & 2.59 & 37.7 & 104. & 2.4 & 1 & 57,61 \\
\hline 81.8 & 5.3 & 12.8 & 4.56 & 1.38 & 3.29 & 63.3 & 2.59 & 52.7 & 77.0 & 2.5 & 3 & 54 \\
\hline 81.8 & 5.3 & 12.8 & 4.56 & 2.37 & 3.29 & 63.3 & 2.59 & 52.7 & 77.0 & 2.5 & 3 & - \\
\hline 81.8 & 5.3 & 12.8 & 4.56 & 2.58 & 3.29 & 63.3 & 2.59 & 52.7 & 77.0 & 2.5 & 3 & 22 \\
\hline 74.4 & 12.1 & 13.6 & 4.25 & 1.28 & 3.17 & 49.2 & 2.40 & 106. & 157. & 3.1 & $3 a$ & - \\
\hline 74.4 & 12.1 & 13.6 & 4.25 & 2.27 & 3.17 & 49.2 & 2.40 & 106. & 157. & 3.1 & $3 a$ & - \\
\hline 74.4 & 12.1 & 13.6 & 4.25 & 2.48 & 3.17 & 49.2 & 2.40 & 106. & 157. & 3.1 & $3 a$ & - \\
\hline 78.4 & 8.1 & 13.5 & 21.7 & 0.48 & 2.22 & 25.4 & 2.00 & 27.9 & 55.0 & 5.6 & 4 & 10,45 \\
\hline 83.8 & 4.2 & 12.0 & 22.2 & 0.41 & 2.25 & 25.3 & 2.00 & 28.9 & 57.5 & 5.5 & 4 & - \\
\hline 80.5 & 6.8 & 12.7 & 24.2 & 0.45 & 2.34 & 24.42 & 1.99 & 24.8 & 50.4 & 5.6 & $4 \mathrm{a}$ & 58,62 \\
\hline 80.5 & 6.8 & 12.7 & 16.5 & 0.04 & 1.78 & 14.46 & 1.92 & 11.2 & 22.1 & 7.8 & $4 \mathrm{~b}$ & 23 \\
\hline 89.3 & 1.7 & 9.0 & 39.2 & 2.68 & 3.48 & 59.1 & 2.56 & 42.3 & 79.3 & 2.6 & 5 & 55 \\
\hline 87.0 & 2.2 & 10.8 & 2.32 & (3) & 2.34 & 24.4 & 1.98 & 8.31 & 15.1 & 5.6 & 7 & 67,71 \\
\hline 87.4 & 2.4 & 10.2 & 2.45 & (3) & 2.41 & 22.9 & 1.94 & 9.22 & 17.1 & 6.3 & $7 \mathrm{a}$ & - \\
\hline 88.0 & 2.4 & 9.6 & 4.26 & (3) & 3.18 & 38.8 & 2.32 & 17.6 & 33.8 & 3.84 & $7 b$ & - \\
\hline 88.2 & 2.6 & 9.2 & 2.15 & (3) & 2.26 & 18.2 & 1.85 & 7.82 & 14.4 & 7.1 & $7 \mathrm{c}$ & - \\
\hline 88.2 & 2.6 & 9.2 & 5.41 & (3) & 3.58 & 43.4 & 2.48 & 11.80 & 23.1 & 2.75 & $7 \mathrm{~d}$ & - \\
\hline 88.0 & 2.9 & 9.1 & 2.04 & (3) & 2.20 & 15.5 & 1.78 & 7.6 & 14.0 & 7.8 & $7 \mathrm{e}$ & 68,72 \\
\hline 88.3 & 3.0 & 8.7 & 3.60 & (3) & 2.92 & 26.1 & 2.10 & 15.1 & 29.1 & 4.5 & $7 \mathrm{f}$ & 27 \\
\hline 87.0 & 2.2 & 10.8 & 1.53 & .144 & 1.90 & 16.7 & 2.01 & 3.58 & - & - & - & A. 2 \\
\hline 84.3 & 4.4 & 11.3 & 1.53 & .166 & 1.90 & 16.7 & 2.01 & 7.31 & - & - & - & A. 3 \\
\hline 83.8 & 1.8 & 14.4 & 9.18 & .156 & 1.67 & 11.2 & 1.80 & 2.0 & 3.2 & 9.1 & 8 & 59,63 \\
\hline 83.8 & 1.8 & 14.4 & 9.18 & .156 & 1.67 & 11.2 & 1.80 & 2.0 & 3.2 & 9.1 & 8 & - \\
\hline 84.6 & 1.2 & 14.2 & 9.18 & .146 & 1.67 & 11.2 & 1.80 & 1.3 & 2.2 & 9.1 & 8 & - \\
\hline 88.7 & 0.7 & 10.6 & 38.1 & (5) & 5.15 & 102. & 3.28 & 10.7 & 43.9 & .95 & 9 & 65,69 \\
\hline 89.2 & 0.7 & 10.1 & 40.2 & (5) & 5.29 & 96.2 & 3.30 & 14.3 & 60.4 & .89 & $9 a$ & 11,46 \\
\hline 89.6 & 0.8 & 9.5 & 42.5 & (5) & 5.43 & 90.5 & 3.33 & 14.7 & 114.7 & .81 & $9 b$ & 66,70 \\
\hline 100. & 0.0 & 0.0 & 572. & .008 & 1.67 & 11.4 & 1.65 & 0.0 & 0.0 & 12. & 10 & 18,81 \\
\hline 100. & 0.0 & 0.0 & 689. & 0.02 & 1.63 & 18.3 & 1.87 & 0.0 & 0.0 & 7.0 & $10 \mathrm{a}$ & 18,82 \\
\hline 88.8 & 1.1 & 10.1 & 32.80 & 0.04 & 2.64 & 22.5 & 1.96 & 4.2 & 15.5 & 5.5 & 11 & $30,12,47$ \\
\hline 89.2 & 0.8 & 10.0 & 32.80 & 0.03 & 2.64 & 22.4 & 1.96 & 2.71 & 11.7 & 4.5 & 11 & - \\
\hline 89.0 & 1.0 & 10.0 & 32.80 & 0.03 & 2.64 & 22.4 & 1.96 & 3.3 & 13.1 & 4.5 & 11 & - \\
\hline 83.7 & 0.4 & 15.9 & 5.88 & 0.01 & 1.59 & 8.55 & 1.65 & 0.31 & $(-)$ & 12. & 12 & 84 \\
\hline 92.8 & 1.0 & 6.2 & 16.1 & 0.33 & 2.92 & 26.1 & 2.10 & 6.0 & 8.7 & 4.4 & 13 & 85 \\
\hline 82.7 & 0.4 & 17.0 & 5.21 & 0.03 & 1.59 & 8.59 & 1.65 & 0.16 & $(-)$ & 12. & 14 & 29,83 \\
\hline 88.3 & 0.9 & 10.8 & 39.0 & 2.17 & 2.92 & 26.2 & 2.10 & 3.8 & 4.88 & 4.4 & 15 & 60 \\
\hline 91.2 & 0.5 & 8.4 & 3.60 & 1.00 & 2.92 & 26.1 & 2.10 & 4.6 & - & 4.4 & 16 & 86 \\
\hline 90.1 & 0.5 & 9.4 & 5.14 & 1.42 & 1.74 & 10.38 & 1.71 & 1.21 & - & 11.5 & $16 a$ & \\
\hline 88.3 & 0.9 & 10.8 & 6.28 & 0.50 & 4.63 & 65.6 & 2.80 & 11.3 & - & 1.4 & 18 & 31 \\
\hline 88.9 & 0.4 & 10.7 & 6.00 & 0.45 & 4.52 & 62.7 & 2.90 & 4.0 & - & 1.4 & $18 \mathrm{a}$ & 73,77 \\
\hline 92.5 & 0.4 & 7.2 & 3.27 & .065 & 2.78 & 18.1 & 1.91 & 1.43 & 16.2 & 6.1 & 19 & 92 \\
\hline 92.5 & 0.4 & 7.2 & 3.27 & .065 & 2.78 & 18.1 & 1.91 & 1.43 & 16.2 & 6.1 & 19 & - \\
\hline 92.5 & 0.4 & 7.2 & 3.27 & .065 & 2.78 & 18.1 & 1.91 & 1.43 & 16.2 & 6.1 & 19 & 32 \\
\hline 96.6 & 0.2 & 3.2 & 21.8 & 0.24 & 2.78 & 18.1 & 1.91 & 1.8 & 5.2 & 6.1 & 20 & - \\
\hline 96.6 & 0.2 & 3.2 & 21.8 & 0.24 & 2.78 & 18.1 & 1.91 & 1.8 & 5.2 & 6.1 & 20 & - \\
\hline 96.7 & 0.2 & 3.1 & 21.9 & 0.24 & 6.37 & 94.7 & 3.5 & 9.1 & 29.0 & 1.48 & $20 \mathrm{a}$ & 87 \\
\hline 96.5 & 0.2 & 3.4 & 27.9 & 0.48 & 1.62 & 6.3 & 1.62 & 0.08 & 0.11 & 13. & $20 \mathrm{~b}$ & 88 \\
\hline 96.5 & 0.2 & 3.4 & 27.9 & 0.48 & 1.62 & 6.3 & 1.62 & 0.08 & 0.11 & 13. & $20 \mathrm{~b}$ & - \\
\hline 96.4 & 0.2 & 3.4 & 12.9 & .001 & 2.69 & 18.3 & 1.88 & 1.3 & 2.6 & 6.5 & 21 & 89 \\
\hline 96.4 & 0.2 & 3.4 & 12.9 & .001 & 2.69 & 18.3 & 1.88 & 1.3 & 2.6 & 6.5 & 21 & - \\
\hline 96.1 & 0.2 & 3.7 & 15.5 & 0.35 & 2.54 & 17.8 & 1.86 & 1.13 & 2.27 & 6.8 & $21 \mathrm{a}$ & 13 \\
\hline 96.1 & 0.2 & 3.7 & 15.5 & 0.35 & 2.54 & 17.8 & 1.86 & 1.13 & 2.27 & 6.8 & $21 \mathrm{a}$ & - \\
\hline 98.2 & 0.1 & 1.7 & 6.47 & .003 & 3.66 & 31.4 & 2.34 & 2.7 & - & 3.1 & 22 & - \\
\hline 98.2 & 0.1 & 1.7 & 6.69 & .039 & 3.05 & 21.7 & 2.06 & 1.75 & - & 6.1 & $22 \mathrm{a}$ & 90 \\
\hline (15) & (16) & (17) & (18) & (19) & (20) & (21) & (22) & (23) & (24) & $(25)$ & (26) & (27) \\
\hline
\end{tabular}

the observed spectra. Labels ${ }^{3},{ }^{4}$, and ${ }^{z}$ in Col. 9 indicate $M_{\mathrm{v}}$-values for LC III, IV, and ZAMS instead of the value from SK82 corresponding to the ST in Col. 4 in the case that there were no comparison spectra of the required LC available. The Cols. 15 to 17 give the relative flux contributions $\left(F_{\text {rel }}\right.$ in the $V^{\mathrm{J}}$-band from photosphere, BL, and disc (Eq. (9)). $\boldsymbol{\aleph}$ is the normalisation factor from Eq. (13). $M_{\star}, L_{\star}$ and $t_{\star}$ have been obtained from the Bernasconi tracks (1996), and the mass accretion rate $\dot{M}$
$3236 \AA$, obtained from ground-based photometric observations in the Walraven $W$ pass-band are indicated by w. A dotted line indicates the model spectrum without the BL contribution, but

is calculated from the model parameters assuming $i=90^{\circ}$ (Sect. 6). The $\dot{M}$-value corrected for $v \sin i$ (only if $i$ is known) is given in Col. 24, if, however, the star is seen "pole-on", i.e. $i \leq 20^{\circ}$, this value was not estimated and is given as (-). 
Table 2. Data of the analysed stars (Part 2).

\begin{tabular}{|c|c|c|c|c|c|c|c|c|c|c|c|c|c|}
\hline \# & PMS star & $\begin{array}{c}\text { IUE } \\
\text { images }\end{array}$ & $\begin{array}{l}\text { Photo- } \\
\text { sphere }\end{array}$ & $\begin{array}{l}\text { Bound. } \\
\text { Layer }\end{array}$ & $\begin{array}{l}T_{\mathrm{d}}^{\max } \\
(\mathrm{K})\end{array}$ & $\boldsymbol{E}^{\text {is }}$ & $\begin{array}{l}V_{\text {obs }}^{\text {iue }} \\
(\mathrm{ma}\end{array}$ & $\frac{M_{\mathrm{v}}}{\text { nitude) }}$ & $V_{\mathrm{s}}^{\mathrm{J}, 0}$ & $R^{\mathrm{cs}}$ & \multicolumn{2}{|c|}{$\begin{array}{cr}d & \delta_{\mathrm{bl}} \\
(\mathrm{pc}) & \left(10^{-3}\right)\end{array}$} & $\begin{array}{c}i \\
\left({ }^{\circ}\right) \\
\end{array}$ \\
\hline 23 & FU Ori & 23-01 & F0 I & B7 Ia & 6340 & 0.06 & 9.30 & -2.00 & 6.50 & 3.60 & 500 & 2.0 & 60 \\
\hline $23 a$ & & 23-01 & F0 I & B7 Ia & 6340 & 0.06 & 9.30 & -1.00 & 7.50 & 2.60 & 500 & 2.0 & 60 \\
\hline $23 b$ & & 23-01 & F2 I & B9 Iab & 6052 & 0.06 & 9.30 & -0.50 & 8.00 & 4.00 & 500 & 2.0 & 60 \\
\hline $23 c$ & & $23-02$ & F0 I & B9 Iab & 6034 & 0.06 & 9.30 & -2.00 & 6.50 & 3.25 & 500 & 20 & 60 \\
\hline 25 & CQ Tau & $25-01$ & F0 III & B9 Iab & 5726 & 0.07 & 10.40 & +1.50 & 6.50 & 5.00 & 100 & 10 & 60 \\
\hline $25 \mathrm{a}$ & & $25-01$ & F0 III & B9 Iab & 5726 & 0.07 & 10.40 & $+2.20^{4}$ & 7.20 & 4.65 & 100 & 10 & 60 \\
\hline $25 b$ & & $25-01$ & F3 III $^{2}$ & B9 Iab & 5396 & 0.07 & 10.40 & +1.70 & 6.70 & 5.00 & 100 & 10 & 60 \\
\hline $25 c$ & & $25-01$ & $\mathrm{~F} 3 \mathrm{III}^{2}$ & B9 Iab & 5396 & 0.07 & 10.40 & $+2.50^{4}$ & 7.40 & 5.00 & 100 & 10 & 60 \\
\hline $25 \mathrm{~d}$ & & $25-02$ & F0 III & B9 Iab & 5834 & 0.07 & 9.80 & +1.50 & 6.50 & 5.25 & 100 & 4.0 & 60 \\
\hline $25 \mathrm{e}$ & & $25-02$ & $\mathrm{~F} 2 \mathrm{III}^{2}$ & B9 Iab & 5606 & 0.07 & 9.80 & $+2.20^{4}$ & 7.20 & 5.50 & 100 & 4.0 & 60 \\
\hline 26 & NX Pup & $26-01$ & $\mathrm{~F} 2 \mathrm{III}^{2}$ & B9 Iab & 5091 & 0.00 & 10.24 & +1.70 & 10.20 & 3.10 & 500 & 80 & 0 \\
\hline 26 & & $26-02$ & $\mathrm{~F} 2 \mathrm{III}^{2}$ & B9 Iab & 5303 & 0.00 & 10.24 & +1.70 & 10.20 & 3.10 & 500 & 30 & 0 \\
\hline 26 & & $26-03$ & $\mathrm{~F} 2 \mathrm{III}^{2}$ & B9 Iab & 5145 & 0.00 & 10.24 & +1.70 & 10.20 & 3.25 & 500 & 60 & 0 \\
\hline 27 & $\mathrm{BD}+46^{\circ} 3471$ & $27-01$ & A3 III & B8 Ia & 6392 & 0.31 & 10.16 & -0.48 & 9.29 & 3.10 & 900 & 60 & 60 \\
\hline 28 & HK Ori & $28-01$ & $\mathrm{~A} 4 \mathrm{~V}$ & B8 Ia & 6292 & 0.06 & 11.54 & $+1.25^{4}$ & 9.52 & 6.50 & 450 & 60 & 0 \\
\hline $28 \mathrm{a}$ & & $28-01$ & A4 V & B5 Ia & 6329 & 0.06 & 11.54 & $+0.60^{3}$ & 8.87 & 6.50 & 450 & 80 & 0 \\
\hline $28 b$ & & $28-01$ & F6 IV & B8 Ia & 4836 & 0.06 & 11.54 & +2.50 & 10.77 & 4.75 & 450 & 80 & 0 \\
\hline $28 \mathrm{c}$ & & $28-01$ & F6 III & B8 Ia & 4838 & 0.06 & 11.54 & +1.60 & 9.87 & 5.25 & 450 & 80 & 0 \\
\hline $28 \mathrm{~d}$ & & $28-01$ & F9 v & B8 Ia & 4691 & 0.06 & 11.54 & $+3.00^{4}$ & 11.27 & 4.00 & 450 & 80 & 0 \\
\hline $28 \mathrm{e}$ & & $28-01$ & F9 v & B8 Ia & 4691 & 0.06 & 11.54 & $+1.10^{3}$ & 9.37 & 5.50 & 450 & 80 & 0 \\
\hline 29 & AK Sco & 29-01 & F6 IV & B9 Iab & 5237 & 0.17 & 8.80 & +2.20 & 8.08 & 3.10 & 150 & 2.0 & 63 \\
\hline 29 & & $29-02$ & F6 IV & B9 Iab & 5223 & 0.17 & 8.95 & +2.20 & 8.08 & 3.10 & 150 & 2.5 & 63 \\
\hline 29 & & $29-03$ & F6 IV & B9 Iab & 5170 & 0.17 & 9.03 & +2.20 & 8.08 & 3.10 & 150 & 5.0 & 63 \\
\hline 30 & Z CMa & $30-01$ & F5 Ib & B9 Iab & 5410 & 0.20 & 9.30 & -2.00 & 8.11 & 5.00 & 1050 & 20 & 60 \\
\hline $30 \mathrm{a}$ & & $30-02$ & F2 I & B9 Iab & 5761 & 0.20 & 9.60 & -2.00 & 8.11 & 3.10 & 1050 & 20 & 60 \\
\hline 31 & HD 199143 & $31-01$ & F6 V & B9 Iab & 5313 & 0.00 & 7.10 & +3.60 & 6.99 & 3.10 & 47.7 & 0.1 & 45 \\
\hline 31 & & $31-02$ & F6 V & B9 Iab & 5313 & 0.00 & 7.10 & +3.60 & 6.99 & 3.10 & 47.7 & 0.1 & 45 \\
\hline 32 & V380 Ori & $32-01$ & A4 V & B8 Ib & 6194 & 0.06 & 10.51 & $+0.50^{3}$ & 8.77 & 6.50 & 450 & 80 & 0 \\
\hline $32 \mathrm{a}$ & & $32-01$ & A $4 \mathrm{~V}$ & $\mathrm{~B} 8 \mathrm{Ib}$ & 6194 & 0.06 & 10.51 & $+1.25^{4}$ & 9.52 & 6.00 & 450 & 80 & 0 \\
\hline $32 b$ & & $32-01$ & F6 IV & B8 Ib & 4836 & 0.06 & 10.51 & +2.50 & 10.77 & 3.10 & 450 & 80 & 0 \\
\hline $32 c$ & & $32-01$ & F9 v & B8 Ib & 4691 & 0.06 & 10.51 & $+3.00^{4}$ & 11.27 & 3.10 & 450 & 80 & 0 \\
\hline 33 & NV Ori & 33-01 & F6 III & B8 Ia & 4998 & 0.06 & 9.86 & +1.30 & 9.57 & 3.10 & 450 & 20 & 60 \\
\hline 35 & KK Oph & $35-01$ & $\mathrm{~A} 5 \mathrm{v}^{1}$ & B5 Ia & 6177 & 0.32 & 11.82 & +1.95 & 8.46 & 6.50 & 200 & 100 & 60 \\
\hline $35 \mathrm{a}$ & & $35-01$ & F0 v & B5 Ia & 5552 & 0.17 & 11.82 & +2.70 & 8.88 & 6.25 & 172 & 40 & 60 \\
\hline $35 b$ & & $35-01$ & F6 V & B5 Ia & 4962 & 0.00 & 11.82 & +3.60 & 9.48 & 6.50 & 150 & 40 & 60 \\
\hline $35 c$ & & $35-01$ & F6 IV & B9 Iab & 4862 & 0.00 & 11.82 & +2.50 & 9.49 & 6.50 & 250 & 40 & 60 \\
\hline 36 & SU Aur & $36-01$ & G2 IV & B9 Iab & 4706 & 0.18 & 9.00 & +2.72 & 8.51 & 3.10 & 144 & 0.5 & 40 \\
\hline $36 a$ & & $36-01$ & G5 IV & B9 Iab & 4544 & 0.18 & 9.00 & +2.72 & 8.51 & 3.10 & 144 & 0.5 & 40 \\
\hline $36-$ & & $36-01$ & G2 IV & F0 I & 4432 & 0.12 & 9.00 & +2.99 & 8.78 & 3.10 & 144 & 20 & 40 \\
\hline $36-$ & & $36-01$ & G5 IV & F0 I & 4279 & 0.12 & 9.00 & +2.80 & 8.59 & 3.10 & 144 & 20 & 40 \\
\hline 38 & HD 283572 & $38-01$ & G5 IV & A0 Ib & 4561 & 0.03 & 9.05 & +3.45 & 8.99 & 3.10 & 128 & 0.2 & 60 \\
\hline 39 & $\mathrm{BD}+09^{\circ} 880$ & $39-01$ & B9V & B9 Iab & 8816 & 0.06 & 9.90 & $+0.85^{z}$ & 9.12 & 7.50 & 450 & 0 & 0 \\
\hline $39 a$ & & $39-01$ & B9 v & B9 Iab & 8221 & 0.06 & 9.90 & $+0.85^{z}$ & 9.12 & 7.50 & 450 & 20 & 0 \\
\hline 39 & & $39-01$ & B9 III & $\mathrm{B} 5 \mathrm{Ib}^{1}$ & 8617 & 0.06 & 9.90 & $+0.00^{4}$ & 8.27 & 7.00 & 450 & 20 & 0 \\
\hline 40 & HD 100546 & $40-01$ & B9 v & B5 Ia & 8227 & 0.00 & 6.67 & +0.20 & 5.26 & 7.50 & 103 & 20 & 51 \\
\hline 41 & HD 98922 & $41-01$ & $\mathrm{~A} 0 \mathrm{~V}$ & B9 Iab & 7455 & 0.00 & 6.80 & $+1.30^{z}$ & 6.60 & 3.10 & 115 & 20 & 60 \\
\hline $41 \mathrm{a}$ & & $41-01$ & A0 IV & B9 Iab & 7764 & 0.00 & 6.80 & +0.30 & 6.58 & 3.10 & 180 & 20 & 45 \\
\hline $41 b$ & & $41-01$ & A1 III & B9 Iab & 7424 & 0.00 & 6.80 & +0.30 & 6.80 & 3.10 & 203 & 20 & 60 \\
\hline 42 & HD 97048 & $42-01$ & B9 v & B9 Iab & 8221 & 0.08 & 8.46 & $+0.85^{z}$ & 7.13 & 3.20 & 180 & 20 & 70 \\
\hline 42 & & $42-01$ & B9 III & B5 Ia & 8617 & 0.08 & 8.46 & $-0.10^{4}$ & 6.18 & 4.10 & 180 & 20 & 85 \\
\hline 42 & & $42-01$ & B9 III & B5 Ia & 8617 & 0.08 & 8.46 & -0.60 & 5.68 & 4.50 & 180 & 20 & 85 \\
\hline 43 & HD 95881 & 43-01 & A1 III & B7 III & 7213 & 0.17 & 8.20 & +0.30 & 7.69 & 4.50 & 300 & 40 & 80 \\
\hline (1) & (2) & (3) & (4) & (5) & (6) & (7) & (8) & (9) & (10) & (11) & (12) & (13) & (14) \\
\hline
\end{tabular}

this line is not always shown, e.g. when the difference is not large enough on the plotted scale.

Also shown for all the individual low-resolution spectra (top - observed (PMS) star, bottom-upper - comparison star and bottom-lower - boundary-layer) are the quality flags as used in ULDA (Wamsteker et al. 1989): extrapolated ITF (+), microphonics $(\circ)$, filtered bright spot $(\star)$, unfiltered bright spot $(*)$, resau mark $(\times)$, saturated $(\triangle)$, outside PHOT region $(\diamond)$, other flag values i.e. MXLO-files ( $\square$ ).
In the following sections we discuss the various groups of related stars separately, as well as some individual stars.

\subsection{Multiple solutions}

Although there is general agreement between UV and visual spectral type, we could match the UV spectra of some stars with more than one ST or LC. Very remarkable cases are HK Ori (A4 - F9), V380 Ori (A4 - F9), and KK Oph (A5 - F6). 
Table 2. Data of the analysed stars (Part 2 continued).

\begin{tabular}{|c|c|c|c|c|c|c|c|c|c|c|c|c|}
\hline \multicolumn{3}{|c|}{$F_{\text {rel }}(5500 \AA) \times 100$} & \multirow{2}{*}{$\begin{array}{c}\boldsymbol{\kappa} \\
\left(10^{-20}\right)\end{array}$} & \multirow{2}{*}{$\begin{array}{c}A_{\mathrm{v}}^{\mathrm{cs}} \\
(\mathrm{mag})\end{array}$} & \multirow{2}{*}{$\begin{array}{c}R_{\star} \\
\left(R_{\odot}\right) \\
\end{array}$} & \multirow{2}{*}{$\begin{array}{c}L_{\star} \\
\left(L_{\odot}\right) \\
\end{array}$} & \multirow{2}{*}{$\begin{array}{c}M_{\star} \\
\left(M_{\odot}\right) \\
\end{array}$} & $\dot{M}$ & $\dot{M}^{\mathrm{c}}$ & \multirow{2}{*}{$\begin{array}{c}t_{\star} \\
(\mathrm{Myr}) \\
\end{array}$} & \multirow[b]{2}{*}{$\#$} & \multirow[b]{2}{*}{ Fig. } \\
\hline star & $\mathrm{BL}$ & disc & & & & & & \multicolumn{2}{|c|}{$\left(10^{-8} M_{\odot} \mathrm{yr}^{-1}\right)$} & & & \\
\hline 91.5 & 0.4 & 8.1 & 37.9 & 2.72 & 11.8 & 442. & 0.5 & 834. & $23.10^{3}$ & - & 23 & - \\
\hline 91.5 & 0.4 & 8.1 & 15.1 & 1.72 & 7.45 & 175. & 0.5 & 200. & $65.10^{3}$ & - & $23 \mathrm{a}$ & 97 \\
\hline 92.3 & 0.3 & 7.4 & 11.5 & 1.21 & 6.53 & 111. & 0.5 & 70.0 & $54.10^{2}$ & - & $23 b$ & - \\
\hline 88.6 & 2.8 & 8.6 & 37.9 & 2.76 & 11.8 & 442. & 0.5 & $83.10^{2}$ & $23.10^{4}$ & - & $23 c$ & - \\
\hline 90.6 & 1.9 & 7.5 & 52.3 & 3.79 & 2.78 & 18.1 & 1.92 & 7.11 & 7.63 & 5.6 & 25 & 91 \\
\hline 90.6 & 1.9 & 7.5 & 27.4 & 3.09 & 2.01 & 9.60 & 1.59 & 3.28 & 7.23 & 15.5 & $25 \mathrm{a}$ & - \\
\hline 90.7 & 2.5 & 6.8 & 55.9 & 3.58 & 2.88 & 15.2 & 1.88 & 8.08 & 19.65 & 5.6 & $25 b$ & - \\
\hline 90.7 & 2.5 & 6.8 & 29.3 & 2.89 & 2.08 & 7.99 & 1.51 & 3.82 & 8.78 & 11.0 & $25 c$ & - \\
\hline 92.0 & 0.8 & 7.2 & 52.3 & 3.17 & 2.78 & 18.1 & 1.92 & 2.86 & 3.07 & 5.6 & $25 d$ & - \\
\hline 92.4 & 1.2 & 6.5 & 32.5 & 2.47 & 2.19 & 9.57 & 1.60 & 1.68 & 3.85 & 10.0 & $25 \mathrm{e}$ & 33,14 \\
\hline 54.3 & 30.8 & 14.8 & 1.55 & 0.71 & 2.76 & 15.2 & 1.86 & 56.6 & $(-)$ & 6.2 & 26 & $1,2,93$ \\
\hline 71.7 & 15.3 & 13.0 & 1.55 & 0.41 & 2.76 & 15.2 & 1.86 & 21.2 & $(-)$ & 6.2 & 26 & - \\
\hline 60.3 & 25.7 & 14.0 & 1.55 & 0.59 & 2.76 & 15.2 & 1.86 & 42.4 & $(-)$ & 6.2 & 26 & 34 \\
\hline 80.2 & 6.7 & 13.1 & 2.07 & 0.15 & 4.98 & 121.3 & 3.38 & 200. & 745. & 0.8 & 27 & 56 \\
\hline 65.1 & 15.1 & 19.8 & 1.37 & 2.30 & 2.34 & 24.4 & 1.97 & 35.0 & $(-)$ & 5.9 & 28 & 25,95 \\
\hline 50.2 & 21.7 & 28.1 & 2.50 & 3.23 & 3.16 & 43.6 & 2.38 & 154.5 & $(-)$ & 3.1 & $28 \mathrm{a}$ & - \\
\hline 42.7 & 39.3 & 18.0 & 1.29 & 1.51 & 2.27 & 7.54 & 1.58 & 52.9 & $(-)$ & 9.3 & $28 b$ & - \\
\hline 42.7 & 39.3 & 18.0 & 2.96 & 2.41 & 3.44 & 17.30 & 2.08 & 138.1 & $(-)$ & 4.2 & $28 \mathrm{c}$ & - \\
\hline 36.6 & 44.7 & 18.7 & 1.08 & 1.17 & 2.08 & 5.39 & 1.50 & 42.7 & $(-)$ & 10.0 & $28 \mathrm{~d}$ & - \\
\hline 36.6 & 44.7 & 18.7 & 6.22 & 3.07 & 4.98 & 31.0 & 2.81 & 314.7 & $(-)$ & 2.3 & $28 \mathrm{e}$ & - \\
\hline 94.0 & 0.6 & 5.4 & 21.1 & 0.26 & 2.61 & 9.96 & 1.70 & 1.34 & 1.52 & 7.6 & 29 & 15,48 \\
\hline 93.9 & 0.8 & 5.4 & 21.1 & 0.41 & 2.61 & 9.96 & 1.70 & 1.67 & 1.89 & 7.6 & 29 & 35 \\
\hline 93.0 & 1.5 & 5.5 & 21.1 & 0.50 & 2.61 & 9.96 & 1.70 & 3.34 & 3.78 & 7.6 & 29 & - \\
\hline 88.9 & 4.2 & 6.9 & 127. & 0.74 & 14.4 & 418. & 1.7: & $22.10^{2}$ & $35.10^{4}$ & - & 30 & - \\
\hline 88.5 & 3.4 & 8.1 & 104. & 0.29 & 13.0 & 443. & 1.7: & $28.10^{2}$ & $78.10^{3}$ & - & $30 \mathrm{a}$ & 98 \\
\hline 92.3 & 0.04 & 7.7 & 49.9 & 0.19 & 1.38 & 2.80 & 1.24 & 0.014 & 0.05 & 17. & 31 & - \\
\hline 92.3 & 0.04 & 7.7 & 49.9 & 0.19 & 1.38 & 2.80 & 1.24 & 0.014 & 0.05 & 17. & 31 & 74,78 \\
\hline 60.7 & 19.0 & 20.3 & 2.74 & 2.10 & 3.31 & 47.8 & 2.45 & 105.7 & $(-)$ & 4.2 & 32 & - \\
\hline 60.7 & 19.0 & 20.3 & 1.37 & 1.35 & 2.34 & 24.4 & 1.97 & 4.67 & $(-)$ & 5.9 & $32 \mathrm{a}$ & 24,16 \\
\hline 42.6 & 39.5 & 17.9 & 1.29 & 0.48 & 2.27 & 7.54 & 1.58 & 52.9 & $(-)$ & 9.3 & $32 b$ & - \\
\hline 36.5 & 45.0 & 18.5 & 1.08 & 0.15 & 2.08 & 5.39 & 1.50 & 42.7 & $(-)$ & 10.0 & $32 \mathrm{c}$ & 39,17 \\
\hline 85.2 & 7.2 & 7.6 & 5.20 & 0.41 & 3.95 & 22.8 & 2.35 & 46.7 & 88.7 & 2.6 & 33 & 94 \\
\hline 60.5 & 14.9 & 24.6 & 5.39 & 2.92 & 1.79 & 12.9 & 1.86 & 59.4 & 97.5 & 7.9 & 35 & 26 \\
\hline 72.6 & 10.9 & 16.5 & 6.00 & 2.76 & 1.62 & 6.29 & 1.61 & 20.5 & 34.1 & 11.9 & $35 a$ & - \\
\hline 65.4 & 16.4 & 18.2 & 5.75 & 2.80 & 1.38 & 2.80 & 1.23 & 16.7 & 28.7 & 19.1 & $35 b$ & - \\
\hline 79.6 & 12.2 & 8.2 & 5.58 & 2.58 & 2.27 & 7.54 & 1.58 & 19.0 & 35.5 & 9.3 & $35 c$ & 36,96 \\
\hline 93.4 & 0.4 & 6.3 & 19.9 & 0.00 & 2.68 & 6.56 & 1.90 & 0.32 & 0.65 & 4.3 & 36 & 19,40 \\
\hline 93.8 & 0.4 & 5.8 & 23.8 & 0.00 & 2.93 & 6.83 & 2.11 & 0.38 & 0.76 & 2.8 & $36 a$ & 75,79 \\
\hline 87.0 & 6.8 & 6.2 & 15.6 & 0.00 & 2.37 & 5.17 & 1.78 & 1.01 & 2.2 & 5.6 & - & 20 \\
\hline 86.2 & 8.1 & 5.7 & 22.1 & 0.20 & 2.82 & 6.37 & 2.07 & 1.87 & 4.11 & 2.9 & - & - \\
\hline 95.9 & 0.1 & 4.0 & 18.1 & 0.02 & 2.09 & 3.48 & 1.70 & 0.055 & 0.15 & 5.3 & 38 & 76,80 \\
\hline 71.6 & 0.0 & 28.4 & 1.16 & 0.96 & 2.15 & 50.3 & 2.56 & 0.0 & 0.0 & 3.5 & 39 & - \\
\hline 69.7 & 2.7 & 27.6 & 1.16 & 0.99 & 2.15 & 50.3 & 2.56 & 4.97 & $(-)$ & 3.5 & $39 a$ & 49 \\
\hline 64.9 & 3.7 & 31.4 & 2.32 & 1.91 & 3.04 & 110.5 & 2.94 & 37.1 & $(-)$ & 3.0 & 39 & - \\
\hline 76.1 & 2.1 & 21.8 & 49.4 & 1.70 & 2.80 & 91.5 & 2.76 & 34.4 & 161. & 2.1 & 40 & 50 \\
\hline 84.8 & 1.5 & 13.7 & 19.2 & 0.38 & 1.94 & 27.6 & 2.19 & 4.25 & - & 5.6 & 41 & 52 \\
\hline 78.6 & 1.7 & 19.7 & 16.2 & 0.49 & 2.97 & 76.8 & 2.63 & 12.8 & - & 2.5 & $41 \mathrm{a}$ & - \\
\hline 84.8 & 1.5 & 13.6 & 15.8 & 0.14 & 3.10 & 69.3 & 2.57 & 14.8 & - & 2.6 & $41 \mathrm{~b}$ & - \\
\hline 85.9 & 0.9 & 13.2 & 10.8 & 1.10 & 2.15 & 50.3 & 2.48 & 5.13 & 10.9 & 3.2 & 42 & 21 \\
\hline 94.1 & 0.5 & 5.4 & 46.4 & 1.95 & 3.19 & 132.9 & 3.12 & 40.3 & 96.8 & 1.6 & 42 & - \\
\hline 94.1 & 0.5 & 5.4 & 46.4 & 2.45 & 4.01 & 210.7 & 3.74 & 67.1 & 150. & 1.8 & 42 & 51 \\
\hline 89.8 & 2.3 & 7.9 & 9.22 & 0.10 & 3.10 & 69.3 & 2.57 & 79.8 & 362. & 2.3 & 43 & 53 \\
\hline (15) & (16) & $\begin{array}{l}(17) \\
\end{array}$ & (18) & (19) & $\begin{array}{l}(20) \\
\end{array}$ & (21) & (22) & (23) & (24) & (25) & (26) & (27) \\
\hline
\end{tabular}

From Table 2 and Figs. 10-17 and 45-110, it may be noted that in the first two cases the relative contribution of the BL in the photometric $V$-band has strongly increased from $\sim 20 \%$ for the early A-types to $\sim 45 \%$ for the F-type solutions. The UV3C calculations suggest that in these cases the later type solutions should be preferred, because they are capable of giving better agreement with the observed spectrum in the long wavelength range of the UV spectrum. More or less the same can be noted for KK Oph, although in this case the increase in the BL contribution for the F-type solutions is not clear. Another example is NX Pup, which was previously classified as B9, but from our UV3C analysis should have an F2 III photosphere (Fig. 9). Also for this star the increase in the contribution of the BL $(\sim 25 \%)$ is significant. The relatively large BL contributions for NX Pup, HK Ori, and V380 Ori are probably favoured by the small inclination angles of their discs. This also explains the presence 


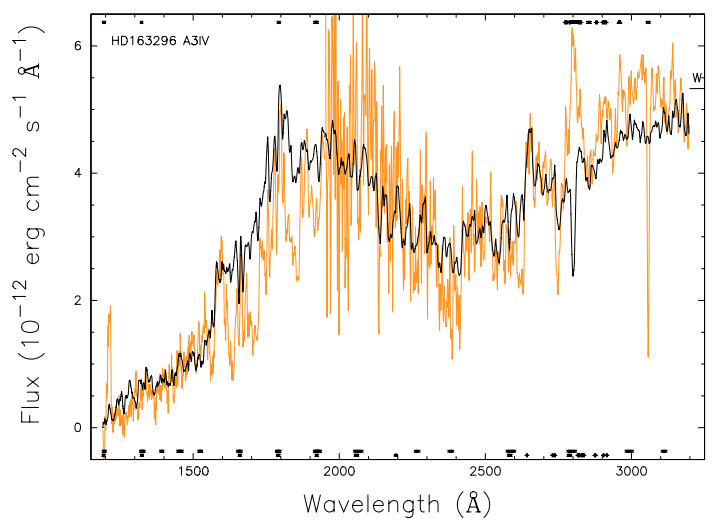

Fig. 10. HD 163296 A3 IV, see also Fig. 45.

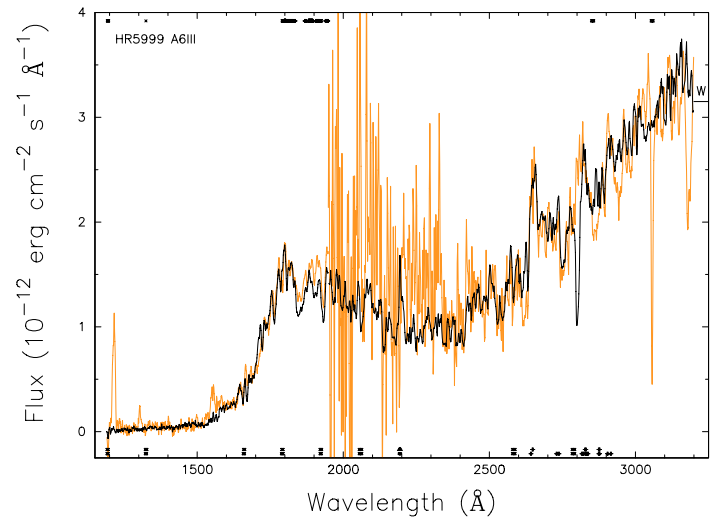

Fig. 11. HR 5999 A6 III, see also Fig. 46.

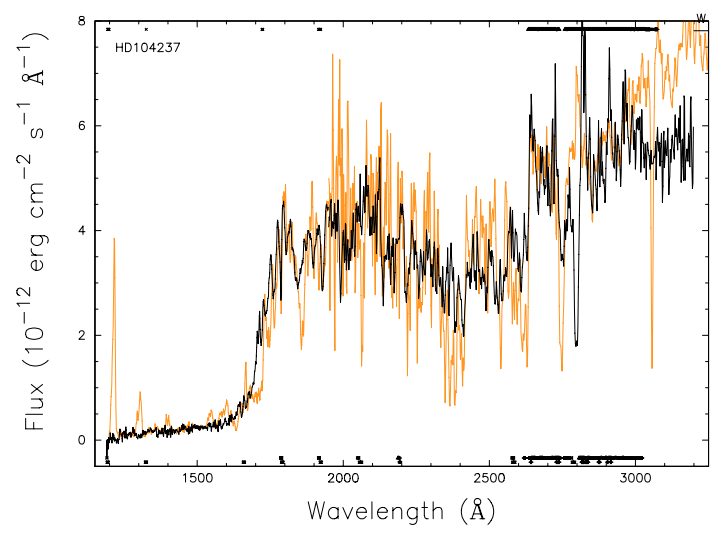

Fig. 12. HD 104237 A7 IV, see also Fig. 47.

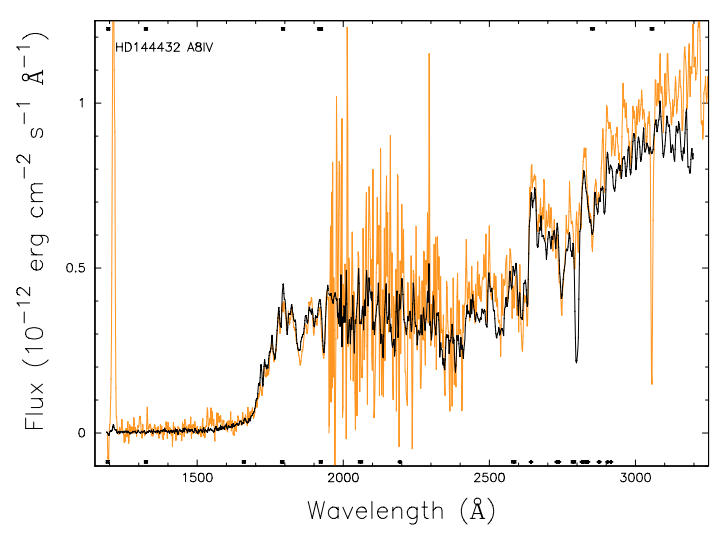

Fig. 13. HD 144432 A8 IV.

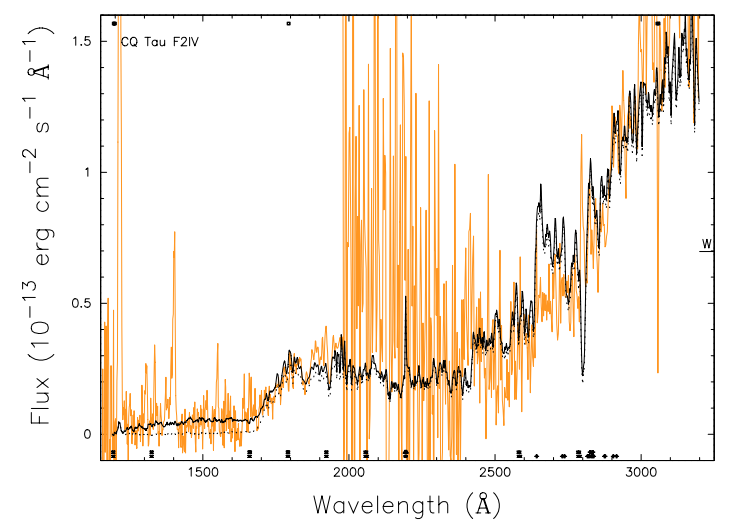

Fig. 14. CQ Tau F2 IV.

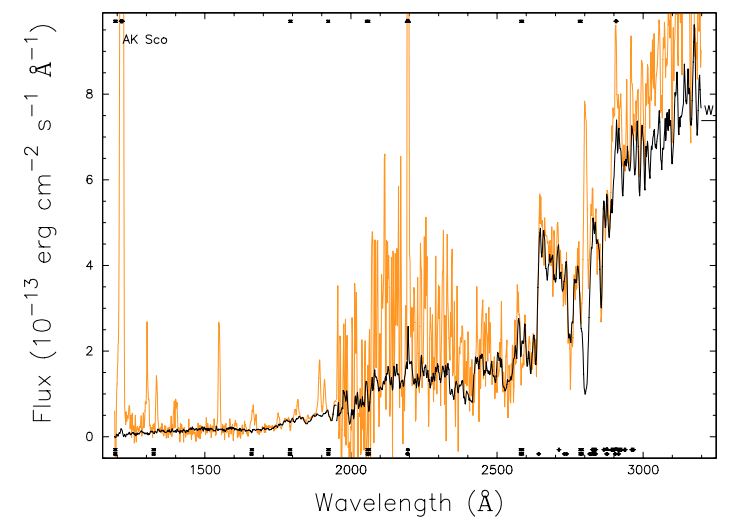

Fig. 15. AK Sco F6 IV, see also Fig. 48.

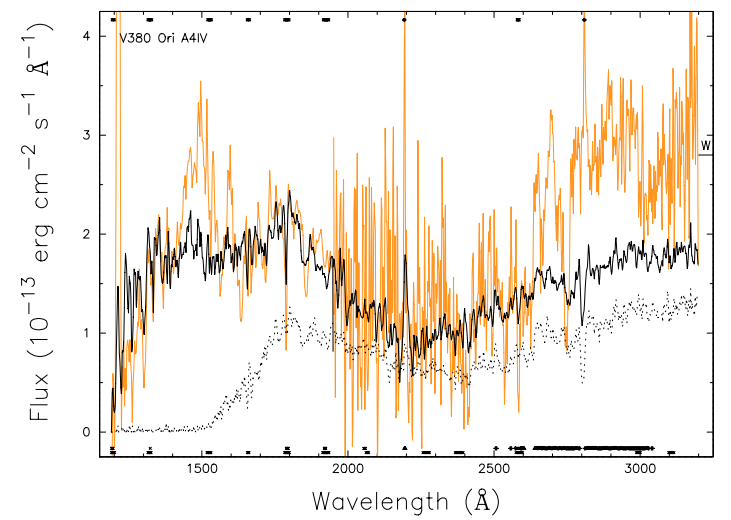

Fig. 16. V380 Ori A4 IV.

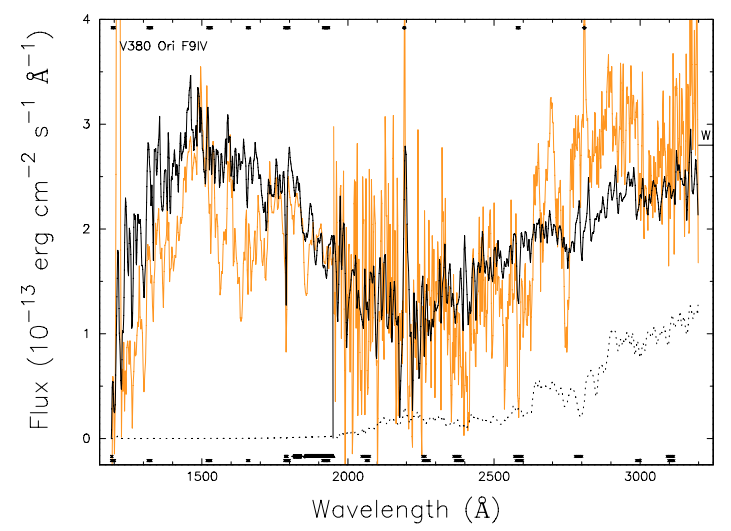

Fig. 17. V380 Ori F9IV. 
Table 3. Additional extinction data of BF Ori; (07-01 top) and (07-02 bot). See Table 2 for the other parameters.

\begin{tabular}{lcccccccc}
\hline \hline & A4 & \multicolumn{2}{c}{ A5 } & \multicolumn{2}{c}{ A6 } & \multicolumn{2}{c}{ A7 } \\
& IV & IV & III & IV & III & IV & III & $V_{\text {obs }}^{\text {iue }}$ \\
\hline$R^{\text {cs }}$ & 5.00 & 5.00 & 5.75 & 5.00 & 6.00 & 4.50 & 5.50 & 11.04 \\
$A_{\mathrm{v}}^{\text {cs }}$ & 1.74 & 1.69 & 2.28 & 1.48 & 2.48 & 1.28 & 1.87 & $(\mathrm{mag})$ \\
\hline$R^{\text {cs }}$ & 5.00 & 5.00 & 6.50 & 5.00 & 6.50 & 4.50 & 6.50 & 9.89 \\
$A_{\mathrm{v}}^{\text {cs }}$ & 0.59 & 0.54 & 1.13 & 0.33 & 1.33 & 0.13 & 0.72 & $(\mathrm{mag})$ \\
\hline
\end{tabular}

of many strong emission lines in the LW-range (hires IUE) of NX Pup and V380 Ori.

In high-resolution optical spectra of V380 Ori, many strong emission-lines of Fe II, [Fe II], Ti II, Cr II, and Ni II have been identified (Shevchenko 1994; Rossi et al. 1999). The origin of these lines is still unknown: they could be formed in the (bipolar) outflow or close to the BL. In the case of KK Oph $\left(i \sim 60^{\circ}\right)$ the emission-lines could be contributed by a nearby nebula.

\subsection{UXORs}

Our model fails to match the spectra of the UXOR-type stars: UX Ori, BF Ori, and CQ Tau over the whole wavelength range of IUE. At maximum brightness the model spectrum agrees well with the observed spectrum, except in the interval 1800-2300 , where the model fluxes are too low. This disagreement seems to increase with lower brightness of the three stars. The parameters of the best matches to the spectra of BF Ori and UX Ori are given in Tables 3 and 4 . The very high values needed for $R^{\mathrm{cs}}$ suggest that the employed c.s. extinction laws are very different from the usual interstellar extinction. This is an indication that UXORs have a peculiar environment of c.s. dust. This problem was studied earlier by Grady et al. (1995), who point to the computations by Voshchinnikov \& Karjukin (1994), which indicate that in order to explain the observed "blueing" of the photometric colours (Fig. D.2) and the simultaneous rise in polarization during the approach to a brightness minimum of UX Ori, one needs a disc $(q=2)$ with (possibly) obsidian grains of a size at least 30 times those of the interstellar medium. The passing of clouds with such large grains through the line of sight may result in strong deviations from the i.s. extinction laws during a phase of low brightness for a $U X O R$.

\subsection{ZAMS stars}

For dwarf stars with ST earlier than F2 V, the values of $M_{\mathrm{v}}$ for ZAMS and MS stars are somewhat different (SK82). For sufficiently accurate distances it is then possible to find out from the UV3C match whether the star is on the ZAMS or on the MS. We find ZAMS positions for: HD 31648, $\beta$ Pic, HD 169142, and HD 139614.

In the case of $\beta$ Pic, the photospheric component clearly agrees better with that of an A7 Vz star (Blondel \& Tjin A Djie 1994) than with that of an A5 Vz star (Kondo \& Bruhweiler 1985), see Fig. 18. For an A8 Vz star (as proposed by Crifo et al. 1997), with $M_{\mathrm{v}}=2.55$ the agreement is very bad, even for $d=18.2 \mathrm{pc}$, which is the maximum possible $\left(A_{\mathrm{v}}^{\mathrm{cs}}=0.0\right)$. Barrado y Navascués et al. (1999) derived $t_{\star}=20 \pm 10 \mathrm{Myr}$ for $\beta$ Pic.

We do not find any contribution from a boundary-layer to the spectrum of $\beta$ Pic, which is consistent with the image of the star, showing that the c.s. dust disc is seen edge on (Smith \& Terrile 1984). However, variable red-shifted absorption components
Table 4. Extinction parameters for 11 observed brightness stages of UX Ori with a photospheric spectral type of A2 III. See Table 2 for the other parameters.

\begin{tabular}{rcccl}
\hline \hline $\begin{array}{c}V_{\text {obs }}^{\text {iue }} \\
(\mathrm{mag})\end{array}$ & $\begin{array}{c}\text { IUE } \\
\text { images }\end{array}$ & $\begin{array}{c}R^{\text {cs }} \\
-\end{array}$ & $\begin{array}{c}A_{\mathrm{v}}^{\text {cs }} \\
(\mathrm{mag})\end{array}$ & $\begin{array}{l}\boldsymbol{E}^{\text {cs }} \\
(\mathrm{mag})\end{array}$ \\
\hline 9.66 & $03-01$ & 5.00 & 1.38 & 0.28 \\
9.71 & $03-02$ & 4.25 & 1.43 & 0.34 \\
9.91 & $03-03$ & 5.25 & 1.63 & 0.31 \\
10.17 & $03-04$ & 5.25 & 1.89 & 0.36 \\
10.65 & $03-05$ & 5.00 & 2.37 & 0.47 \\
10.71 & $03-06$ & 5.25 & 2.43 & 0.46 \\
10.81 & $03-07$ & 5.00 & 2.53 & 0.51 \\
10.86 & $03-08$ & 5.50 & 2.58 & 0.47 \\
11.21 & $03-09$ & 6.00 & 2.93 & 0.49 \\
11.57 & $03-10$ & 6.50 & 3.29 & 0.51 \\
11.91 & $03-11$ & 6.50 & 3.63 & 0.56 \\
\hline
\end{tabular}

in several lines of ionised metals were observed in the visual (Vidal-Majar et al. 1994), which points to episodic infall of matter probably indicating that the accretion disc in $\beta$ Pic has already disappeared.

The A7 stars HD 139614 and HD 169142 appear to have similar mass and age as $\beta$ Pic, and therefore are also classified as A7 vz stars.

\section{4. $T$ Tauri stars}

Two T Tauri stars have been included in our sample: SU Aur and HD 283572. The spectrum of SU Aur has been analysed in order to check if we find results that are comparable with those of BBB88. Figure 20 shows that for parameters similar to those of Bertout et al. (BBB88-Table $4 a)$, we find a good match to the UV spectrum of this star. For $d=160 \mathrm{pc}$ and $i=40^{\circ}$ we derive the following from our UV3C-model calculation with a G2 IV star and an F0 I-type BL $\left(\delta_{\mathrm{bl}}=0.02\right): R_{\star}=2.70 R_{\odot}, L_{\star}=6.68 L_{\odot}$ for $A_{\mathrm{v}}=0.43$, so $M_{\star}=1.97 M_{\odot}$. With $v \sin i=66 \mathrm{~km} \mathrm{~s}^{-1}$, we then obtain an accretion rate of $5.1 \times 10^{-8} M_{\odot} \mathrm{yr}^{-1}$, which is about the same as derived by BBB88. However, the SW-spectrum of SU Aur is very faint and does not permit us to discriminate between various spectral types for the BL. Furthermore, we have no clear argument for choosing a cooler BL (as done by BBB88 for $T_{\text {eff }}=7940 \mathrm{~K}$, which is much lower than for all the other cases in our sample); so if we use a B9 Iab BL of $0.0005 R_{\star}$ (Fig. 19, Table 2), then the mass accretion rate decreases to $6.5 \times$ $10^{-9} M_{\odot} \mathrm{yr}^{-1}$. The $i=62^{\circ}$ determined by Akeson et al. (2002) does not change our result significantly if we use $M_{\mathrm{v}}=2$ m.70 instead of 2. 72 . Note that a photospheric type G5 IV improves the calculated spectrum at $\lambda>2900 \AA$ (Fig. 75) and that a B9 Iab BL gives a better result at $\lambda<1800 \AA$ (Fig. 79). The SED calculated from these parameters is shown in Sect. 5.10 and appears to agree with the (dereddened) observed SED.

For the other T Tauri star HD 283572, we could not find comparable previous UV spectral analyses. This nTT star has a smaller radius and about half the luminosity, and it should be less evolved than SU Aur (Walter et al. 1987). We estimate that the width of its (cooler) BL is about half the width of the BL of SU Aur, which implies that the accretion rate (after accounting for the rotation of this star) may be $\sim 5$ times smaller than that of SU Aur. 

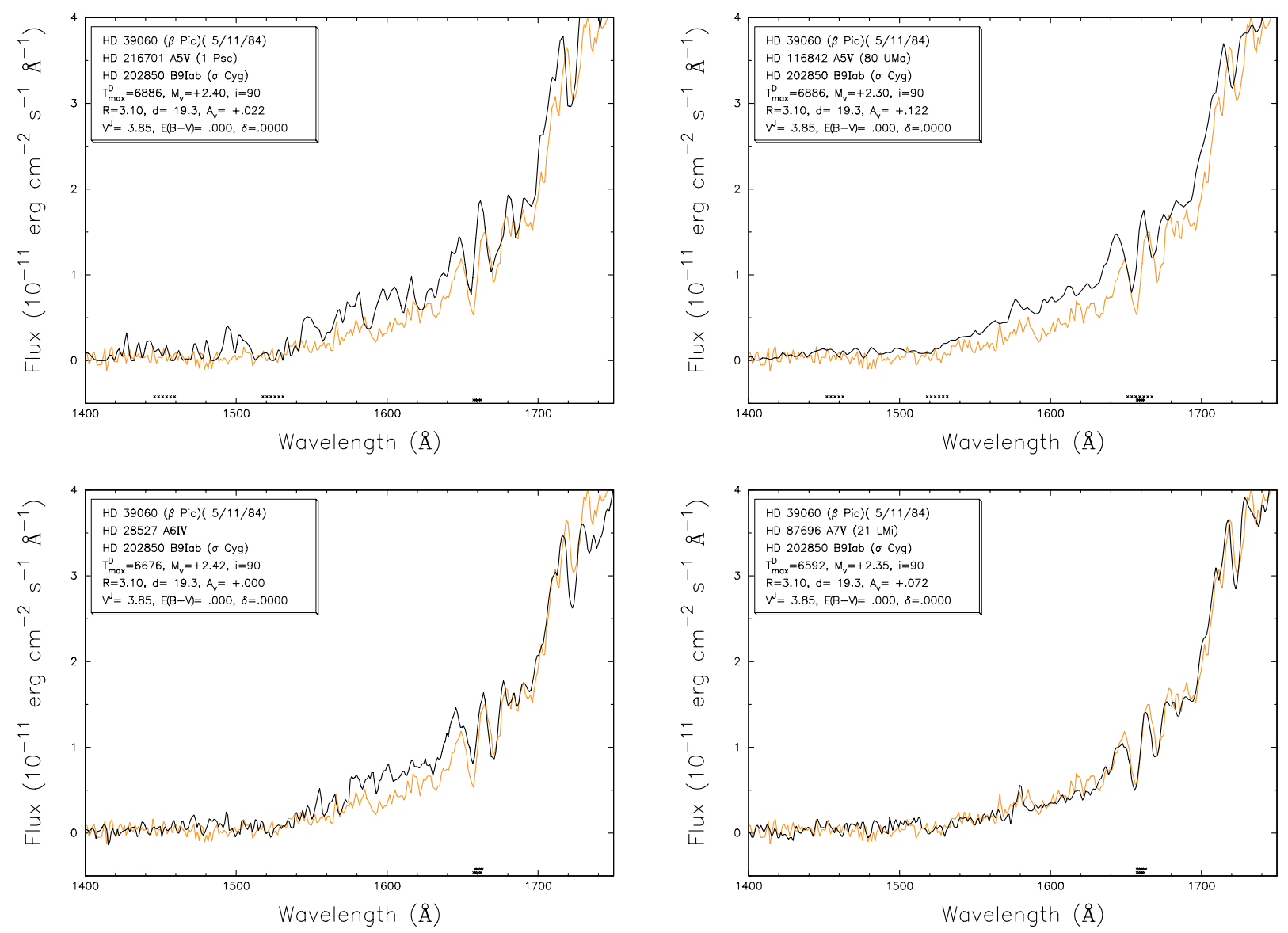

Fig. 18. Enlarged part of the SW-range of $\beta$ Pic solutions for $d=19.3 \mathrm{pc}$. The $M_{\mathrm{v}}$-values are based on agreement of the whole spectrum, except for A6 IV where $M_{\mathrm{v}}$ should be 2. 50, but then $A_{\mathrm{v}}<0$. The $M_{\mathrm{v}}$ ZAMS-values listed by SK82 are 2.1 (A5), 2.25 (A6), and 2.40 (A7).

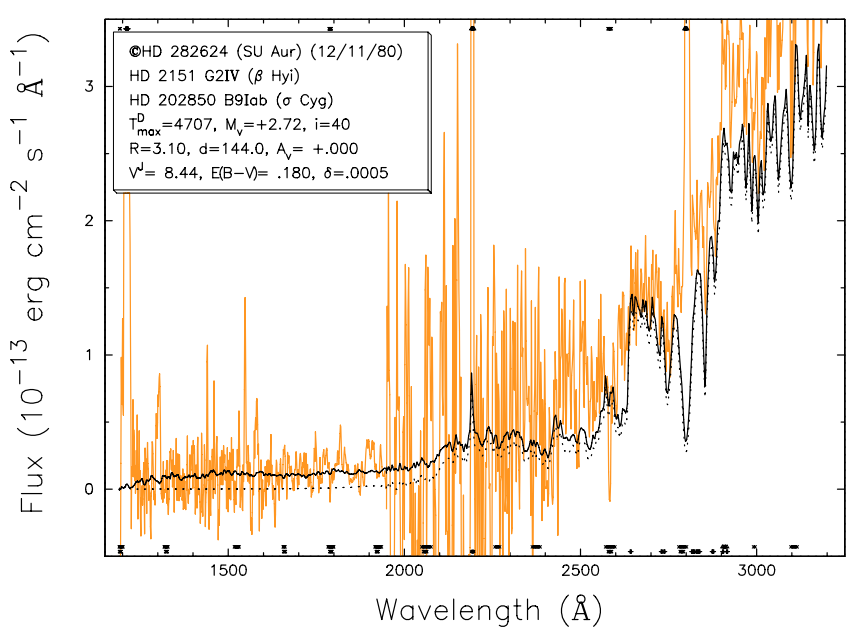

Fig. 19. SU Aur "solution" (G2 IV+ B9 Iab).

\subsection{FUORs}

In contrast to the results in Sect. 5.4, the application of our analysis to the UV spectra of FU Ori and Z CMa leads to very high mass accretion rates. Kenyon et al. (1988) and Hartmann et al. (1989) have convincingly argued that the energy distributions of these stars in the red and IR are dominated by the contributions of a thick rotating accretion disc. In the UV, several absorption features in the LW-range suggest a spectral type F0-5 I for the central star or inner disc (Kenyon et al. 1989). We modeled the

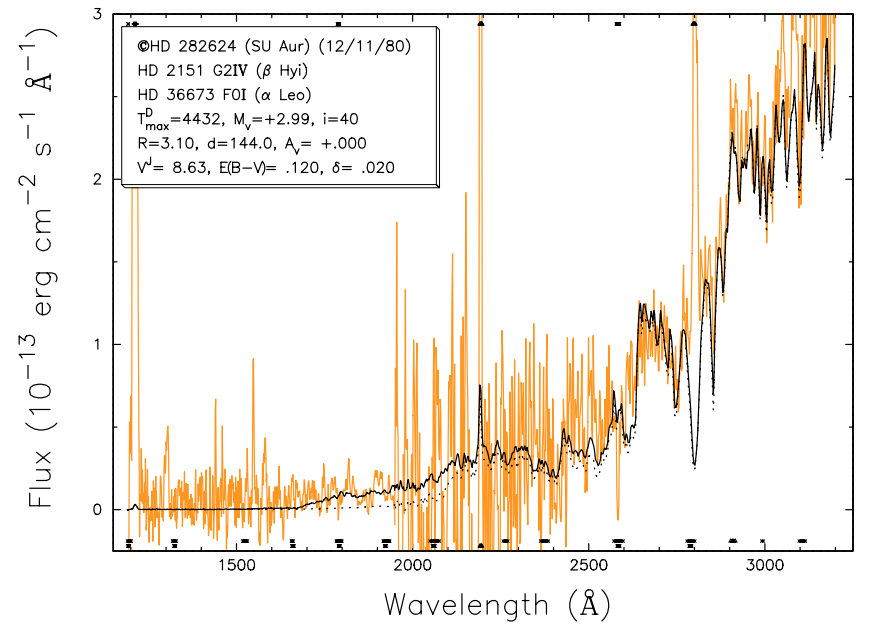

Fig. 20. SU Aur "solution" (G2 IV+ F0 Ib) for parameters close to those of BBB88.

complete UV (SW+LW) spectra of FU Ori and Z CMa with a stellar spectrum of type F0-5 I with $R_{\star} \sim 7 R_{\odot}$ for FU Ori and $R_{\star} \sim 14 R_{\odot}$ for Z CMa (as estimated from the SEDs by Kenyon et al. 1988 and Hartmann et al. 1989). In order to obtain such radii, we had to increase the $M_{\mathrm{v}}$-values from -5.1 (SK82) up to -2.0 . Good agreement with the observed spectra could be obtained with a B7-9 I type BL of $\sim 0.02 R_{\star}$. Because the positions of the stars in the HRD are above the birthline (Fig. 41), we cannot estimate their masses or ages. Assuming $M_{\star} \sim 0.5 M_{\odot}$ for 
Table 5. Additional data of HR 5999. The $V_{\text {obs }}^{\text {iue }}$-value marked with ( $\left.\dagger\right)$ is uncertain, as the brightness is underestimated due to the degradation of the FES counter after 1990 (Sect. B.1). See Table 2 for the other input parameters. The apparently good agreement of the 3 faint spectra (\#10-12) at the long wavelength is caused by the fact that we used the same (overexposed) image LWR14027 in these cases.

\begin{tabular}{rlcccccr}
\hline \hline$\#$ & $V_{\text {obs }}^{\text {iue }}$ & IUE & $R^{\text {cs }}$ & \multicolumn{3}{c}{$A_{\mathrm{v}}^{\text {cs }}(\mathrm{mag})$} & Fig. \\
\cline { 5 - 6 } & $(\mathrm{mag})$ & images & - & A5III & A6III & A7III nn & $(\mathrm{A6})$ \\
\hline 1 & 6.72 & $09-01$ & 3.10 & 0.59 & 0.58 & 0.48 & 99 \\
2 & $6.83 \dagger$ & $09-02$ & 3.50 & 0.70 & 0.69 & 0.59 & 100 \\
3 & 6.81 & $09-03$ & 3.75 & 0.68 & 0.67 & 0.57 & 28,101 \\
4 & 7.02 & $09-04$ & 4.50 & 0.89 & 0.88 & 0.78 & 102 \\
5 & 7.16 & $09-05$ & 4.50 & 1.03 & 1.02 & 0.92 & 103 \\
6 & 7.17 & $09-06$ & 4.50 & 1.04 & 1.03 & 0.93 & 104 \\
7 & 7.20 & $09-07$ & 4.50 & 1.07 & 1.06 & 0.96 & 105 \\
8 & 7.30 & $09-08$ & 4.50 & 1.17 & 1.16 & 1.06 & 106 \\
9 & 7.39 & $09-09$ & 5.00 & 1.26 & 1.25 & 1.15 & 107 \\
10 & 7.47 & $09-10$ & 5.25 & 1.34 & 1.33 & 1.23 & 108 \\
11 & 7.49 & $09-11$ & 5.50 & 1.36 & 1.35 & 1.25 & 109 \\
12 & 7.57 & $09-12$ & 5.75 & 1.44 & 1.43 & 1.33 & 110 \\
\hline
\end{tabular}

FU Ori (a former T Tauri star) and 1.7 $M_{\odot}$ for Z CMa (Hartmann et al. 1989), we then obtain accretion rates of $6 \times 10^{-6} M_{\odot} \mathrm{yr}^{-1}$ for FU Ori and $\sim 3 \times 10^{-5} M_{\odot} \mathrm{yr}^{-1}$ for Z CMa. However, taking the inclinations into account, the mass accretion rates become $6 \times 10^{-4}$ and $8 \times 10^{-4} M_{\odot} \mathrm{yr}^{-1}$. Z CMa is a double star, probably consisting of a $\sim 16 M_{\odot}$ B0 III primary and a $\sim 3 M_{\odot} F U O R$ secondary (van den Ancker et al. 1904). Since the UV spectrum is dominated by the FUOR component we neglected the contribution of the embedded B0-component that only contributes in the far IR.

\subsection{HR 5999}

In Table 2 the parameters of this variable star are given for its phase of maximum visual brightness. The UV3C calculations give the best match for a photospheric spectral type A6 IV-III, but A5 III and A7 III also give satisfactory results. The c.s. extinction is close to the average i.s. extinction law with $R=3.1$. Apart from a low amplitude ( $\delta$ Scuti) oscillation in brightness, the star varies strongly $\left(\Delta V^{\mathrm{J}} \sim 1^{\mathrm{m}}\right.$. $)$ and irregularly on time-scales of several weeks (see e.g. Baade \& Stahl 1989a; Tjin A Djie et al. 1989).

We tried to match the UV spectra of 12 phases of lower brightness with our model. In order to match the decreasing slope of the spectrum longward of $2500 \AA$, we have to raise the value of $R^{\mathrm{cs}}$. The values of $R^{\mathrm{cs}}$ and $A_{\mathrm{v}}^{\mathrm{cs}}$ for fixed values (Table 2) of the other parameters are listed in Table 5. The rise in $R^{\mathrm{cs}}$ can be interpreted roughly as an increase in the average size of the obscuring c.s. dust grains. This may be expected as a result of the passage of c.s. dust clouds with large dust grains through the line of sight. However, the UV spectra longward of $2500 \AA$ for photometric phases with $V^{\mathrm{J}}>6.7$ cannot completely be matched by an increase of $R^{\mathrm{cs}}$ only. Apparently the shape of the extinction law also has to be changed.

A quantitative evaluation of the variation in the extinction law has been made through multiple scattering calculations (Monte Carlo method) for the c.s. dust region by Voshchinnikov \& Karjukin (1994). The results for HR 5999 have been given by Thé et al. (1996). For the brightest phase $\left(V^{\mathrm{J}} \sim 6^{\mathrm{m}} .8\right)$ the extinction law is produced by scattering from grains with sizes between $0.01 \mu \mathrm{m}$ and $0.25 \mu \mathrm{m}$ with $N_{\mathrm{Si}} / N_{\mathrm{C}}=0.75$, which is close to the standard composition of i.s. grains. For the fainter phases, however, the observed extinction law requires that $N_{\mathrm{Si}} / N_{\mathrm{C}} \approx 6.0$, and the sizes range from $0.1 \mu \mathrm{m}$ to $0.13 \mu \mathrm{m}$, which means that the small grains are depleted, and that $R^{\mathrm{cs}} \approx 2.5$. The depletion of small grains has been interpreted as the sweeping effect of the stellar radiation (Thé et al. 1996). At the same time, the EWs of the Fe II, Mn II, and Cr II resonance absorption lines in the inner shell decrease when the star grows fainter (Blondel et al. 1989).

\section{7. $H D 163296$}

The UV spectra of HD 163296 can be matched with an ST A3-4 IV for $d=122 \mathrm{pc}$ (ESA 1997). In order to obtain a good match with A4 Vz $\left(M_{\mathrm{v}}=1 \mathrm{~m} .85\right)$, however, we are limited to $d \leq 111 \mathrm{pc}$ (when using an A0 Ib BL of $0.06 R_{\star}$ ), close to the Hipparcos catalogue lower limit.

\subsection{B9-A1 stars}

The parameters obtained by matching the spectra of five B9/A1 stars are given in Table 2. Because of the large photospheric contribution in the 1200-1500 $\AA$ spectral range of these stars, these spectra are less sensitive to the contribution of their BL, which results in an increasing uncertainty in the BL spectral type and accretion mass rate for spectral types earlier than A0. In fact this problem hampers the application of the UV3C matching procedure to the spectra of Herbig stars with type earler than B9.

\subsection{Double star spectra}

Table B. 1 collects the parameters for 13 (out of 15) stars of our sample, which are known to be double. It is important to check whether the model parameters of the primary component are different from those of the system determined above (Table 2). The results of the attempts to match the spectra of 5 of these systems - XY Per, NX Pup, BD+46 3471 , AK Sco, and KK Oph - are given in Table 6. In most cases we assume that the difference in $V^{\mathrm{J}}$-magnitude of the components at the time of the IUE observation is the same as the literature value of Table B.1 and subtract the relative spectral contribution of the primary component $f_{*}^{\mathrm{p}}=F_{\mathrm{v}}^{\mathrm{p}} / F_{\text {obs }}$ (derived from its total visual magnitude, the difference in magnitude, and the model parameters of the star in Table B.1) from the observed spectrum. The remaining spectrum is assumed to be that of the secondary and is matched separately. These 6 double stars are the ones with the smallest magnitude differences between their components. For the other stars, the differences in $V^{\mathrm{J}}$ are too large to expect a significant contribution in the UV from the secondary.

For XY Per we tried two values for the distance: $350 \mathrm{pc}$ and 120 pc (Sect. C.1). The first value was taken from RSF Per $B$ to which the star seems to belong, while the second is a very uncertain determination by the Hipparcos program. It appears that we can only find a double-star solution for $d=350 \mathrm{pc}$.

Only in the case of the spectroscopic binary AK Sco could we derive the magnitudes of the components at the times of the IUE spectral exposures. These observations were made at apastron (April 1st 1988: $\Delta V^{\mathrm{J}}=0.42$ ) and at periastron (April 9th 1988: $\left.\Delta V^{\mathrm{J}}=0.09\right)$. The magnitudes could be derived from the relative contributions of the two high-resolution $\mathrm{Mg}$ II resonance line profiles of the components, taken on the same dates. We assumed that the primary has spectral type F5 V and then matched the remaining spectrum of the secondary with the same spectral type F5 V (Sect. C.29). 
Table 6. Data of the double stars, p for primary, s for secondary, $f_{\star}$ is the relative $V$-flux of the component.

\begin{tabular}{|c|c|c|c|c|c|c|c|c|c|c|c|c|c|}
\hline \multirow[b]{2}{*}{ PMS star } & \multirow{2}{*}{$f_{\star}$} & \multirow{2}{*}{$\begin{array}{c}\text { IUE } \\
\text { images }\end{array}$} & \multirow{2}{*}{$\begin{array}{l}\text { Photo- } \\
\text { sphere }\end{array}$} & \multirow{2}{*}{$\begin{array}{l}\text { Bound. } \\
\text { Layer }\end{array}$} & \multirow{2}{*}{$\begin{array}{l}T_{\mathrm{d}}^{\max } \\
(\mathrm{K})\end{array}$} & $\boldsymbol{E}^{\text {is }}$ & $V_{\text {obs }}^{\text {iue }}$ & $M_{\mathrm{v}}$ & 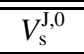 & \multirow{2}{*}{$R^{\mathrm{cs}}$} & \multirow{2}{*}{\multicolumn{2}{|c|}{$\begin{array}{cc}d & \delta_{\mathrm{bl}} \\
(\mathrm{pc}) & \left(10^{-3}\right)\end{array}$}} & \multirow{2}{*}{$\begin{array}{c}i \\
\left({ }^{\circ}\right) \\
\end{array}$} \\
\hline & & & & & & \multicolumn{4}{|c|}{ (magnitude) } & & & & \\
\hline \multicolumn{14}{|c|}{$1-\mathrm{XY}$ Per } \\
\hline $\mathrm{p}$ & .635 & 01-01 & A2 III & B8 Ib & 7050 & 0.19 & 9.87 & +0.30 & 8.02 & 3.85 & 350 & 20 & 60 \\
\hline s1 & .365 & 01-01 & A2 III & B8 Ib & 7049 & 0.19 & 10.47 & +0.30 & 8.02 & 4.40 & 350 & 20 & 60 \\
\hline s2 & .365 & 01-01 & A3 III & B8 Ib & 6738 & 0.19 & 10.47 & +0.50 & 8.22 & 5.00 & 350 & 20 & 60 \\
\hline s3 & .365 & 01-01 & A5 $\mathrm{III}^{2}$ & B8 Ib & 6152 & 0.19 & 10.47 & +0.70 & 8.42 & 5.50 & 350 & 40 & 60 \\
\hline \multicolumn{14}{|c|}{$26-$ NX Pup } \\
\hline $\mathrm{p}$ & .643 & $26-01$ & $\mathrm{~F} 2 \mathrm{III}^{2}$ & B9 Iab & 5237 & 0.00 & 10.72 & +1.70 & 10.20 & 4.50 & 500 & 40 & 0 \\
\hline s1 & .357 & $26-01$ & $\mathrm{~F} 2 \mathrm{III}^{2}$ & B9 Iab & 5237 & 0.00 & 11.36 & +1.70 & 10.20 & 5.25 & 500 & 40 & 0 \\
\hline s2 & .357 & $26-01$ & G5 IV & B9 Iab & 4398 & 0.00 & 11.36 & +3.10 & 11.60 & 3.10 & 500 & 8.0 & 0 \\
\hline $\mathrm{p}$ & .643 & $26-02$ & $2 \mathrm{III}^{2}$ & Iab & 5091 & 0 & 10.72 & +1.70 & 10.20 & 3.85 & 500 & 80 & 0 \\
\hline s1 & .357 & $26-02$ & $\mathrm{~F} 2 \mathrm{III}^{2}$ & $9 \mathrm{Iab}$ & 5237 & 0 & 11.36 & +1.70 & 10.20 & 5.00 & 500 & 40 & 0 \\
\hline s2 & .357 & $26-02$ & G5 IV & B9 Iab & 4411 & 0.00 & 11.36 & +3.00 & 11.50 & 3.10 & 500 & 7.0 & 0 \\
\hline \multicolumn{14}{|c|}{$27-\mathrm{BD}+46^{\circ} 3471$} \\
\hline p1 & .532 & $27-01$ & 3 III & 38 Ia & 6392 & 0.3 & 10.97 & +0.20 & 9.97 & 3.10 & 900 & 60 & 60 \\
\hline s1 & .468 & $27-01$ & $\mathrm{~F} 3 \mathrm{III}^{2}$ & B8 Ib & 5090 & 0.3 & 10.98 & +0.60 & 10.37 & 3.10 & 900 & 60 & 60 \\
\hline $\mathrm{s} 2$ & .468 & $27-01$ & F6 III & B9 Iab & 4766 & 0.31 & 10.98 & +0.68 & 10.45 & 3.10 & 900 & 60 & 60 \\
\hline \multicolumn{14}{|c|}{29 - AK Sco - apastron } \\
\hline $\mathrm{p} 1$ & .595 & $29-02$ & F6 IV & B9 Iab & 5223 & 0.1 & 9.51 & +2.85 & 8.73 & 3.10 & 150 & 2.5 & 63 \\
\hline s1 & .405 & $29-02$ & F6 IV & B9 Iab & 5270 & 0.17 & 9.93 & +2.85 & 8.73 & 5.50 & 150 & 1.0 & 63 \\
\hline \multicolumn{14}{|c|}{29 - AK Sco - periastron } \\
\hline p2 & .522 & $29-03$ & F6 IV & B9 Iab & 5170 & 0.1 & 9.74 & +2.85 & 8.73 & 3.10 & 150 & 5.0 & 63 \\
\hline s2 & .478 & $29-03$ & F6 IV & B9 Iab & 5129 & 0.17 & 9.83 & +2.85 & 8.73 & 3.10 & 150 & 7.5 & 63 \\
\hline \multicolumn{14}{|c|}{35 - KK Oph } \\
\hline p1 & .715 & $35-01$ & $\mathrm{~A} 5 \mathrm{v}^{2}$ & B5 Ia & 6177 & 0.32 & 12.18 & +1.95 & 8.46 & 6.50 & 200 & 100 & 60 \\
\hline s1 & .285 & $35-01$ & F6 IV & B5 Ia & 5243 & 0.32 & 13.18 & +2.50 & 9.01 & 6.25 & 200 & 100 & 60 \\
\hline p2 & .715 & $35-01$ & F6 IV & B9 Iab & 4862 & 0.00 & 12.18 & +2.50 & 9.49 & 6.50 & 250 & 40 & 60 \\
\hline s2 & .285 & $35-01$ & K1 IV & B9 Iab & 3774 & 0.00 & 13.18 & +3.10 & 10.09 & 5.75 & 250 & 40 & 60 \\
\hline & (2) & (3) & (4) & (5) & (6) & (7) & 3) & (9) & (10) & (11) & (12) & (13) & (4) \\
\hline
\end{tabular}

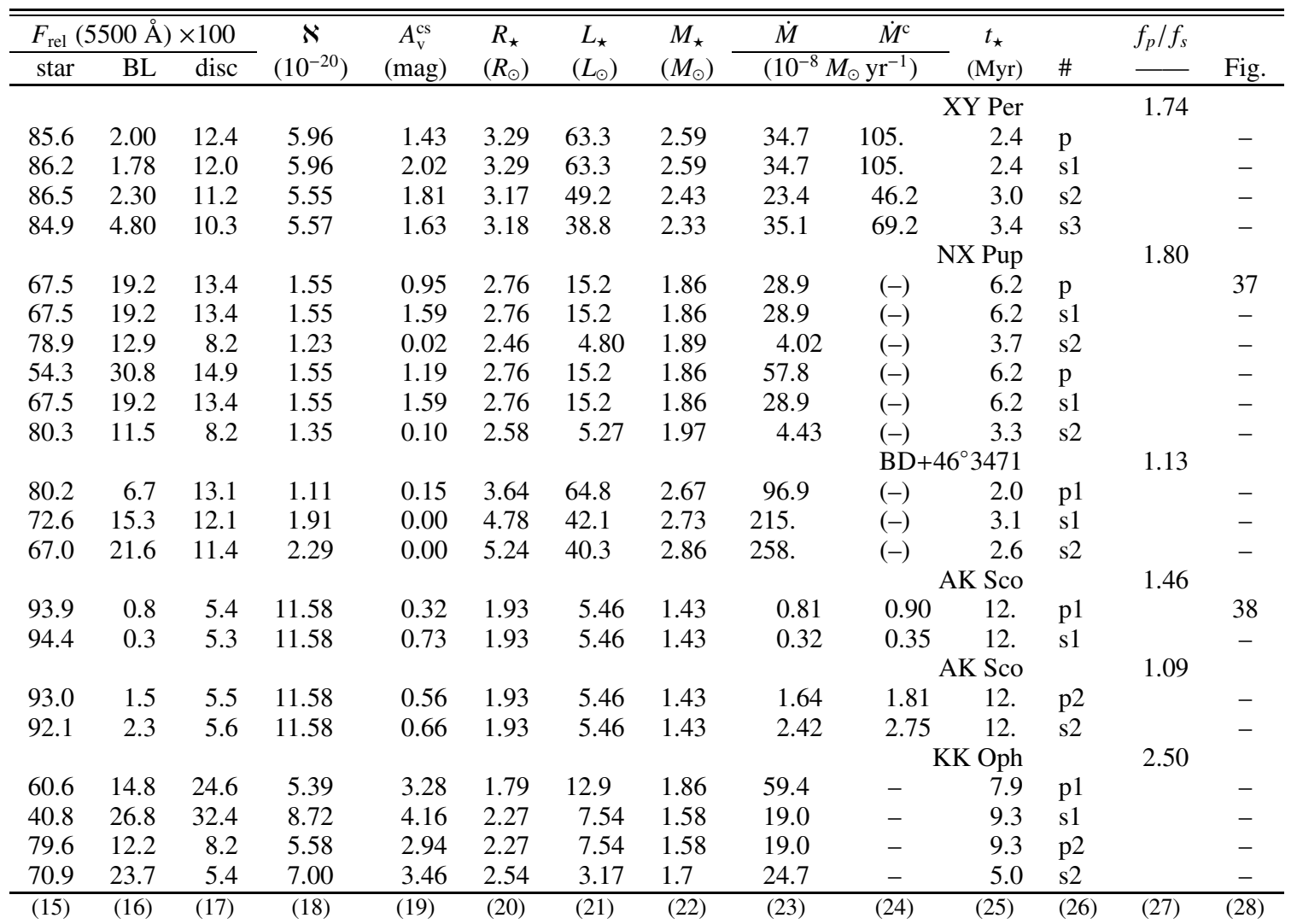

From a comparison between Tables 2 and 6 we note that dominates the UV and Visual (XY Per, NX Pup, and KK Oph; the parameters for those stars where the primary component $f^{\mathrm{P}} / f^{\mathrm{s}}>1.6$ ) are almost the same. But for AK Sco and 
P. F. C. Blondel and H. R. E. Tjin A Djie: Modeling of PMS Ae/Fe stars using UV spectra

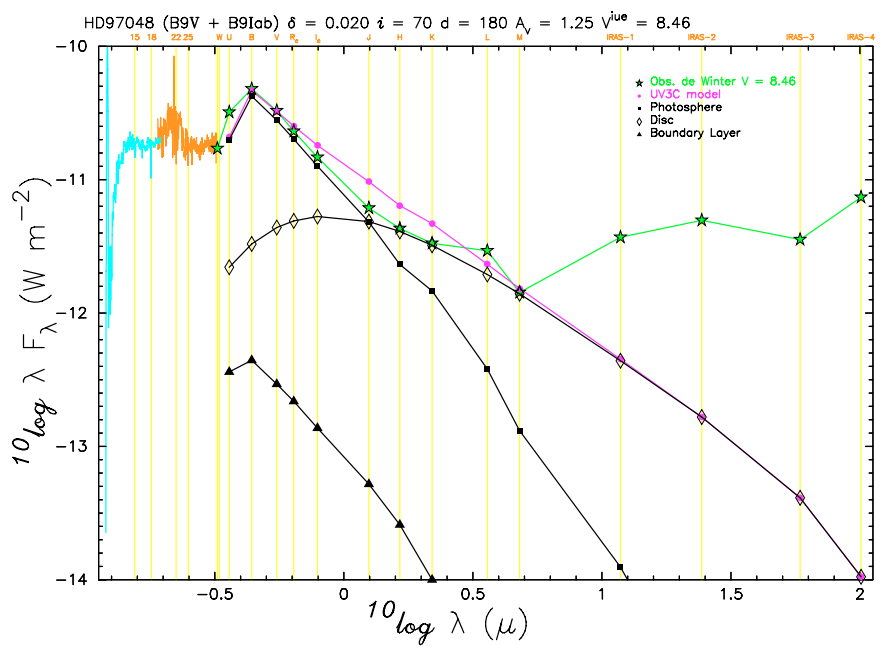

Fig. 21. HD 97048 B9 v.

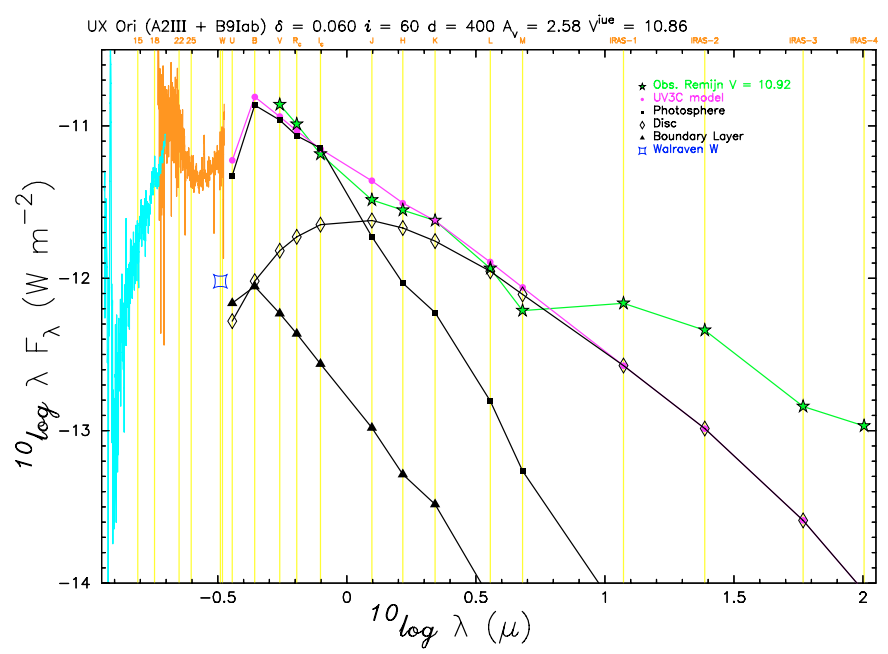

Fig. 22. UX Ori A2 III.

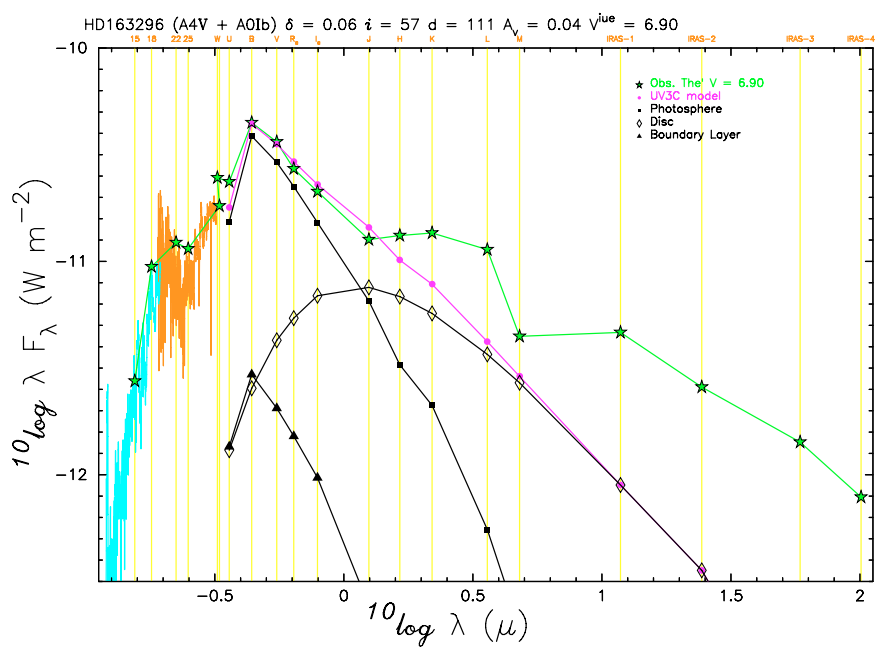

Fig. 23. HD 163296 A4 v.

$\mathrm{BD}+46^{\circ} 3471\left(1.0<f^{\mathrm{p}} / f^{\mathrm{s}}<1.5\right)$, we find smaller $R_{\star}, L_{\star}, M_{\star}$, and $\dot{M}$-values than in Table 2, where these stars were assumed to be single.

These results are important for the understanding of the individual components of the double stars.

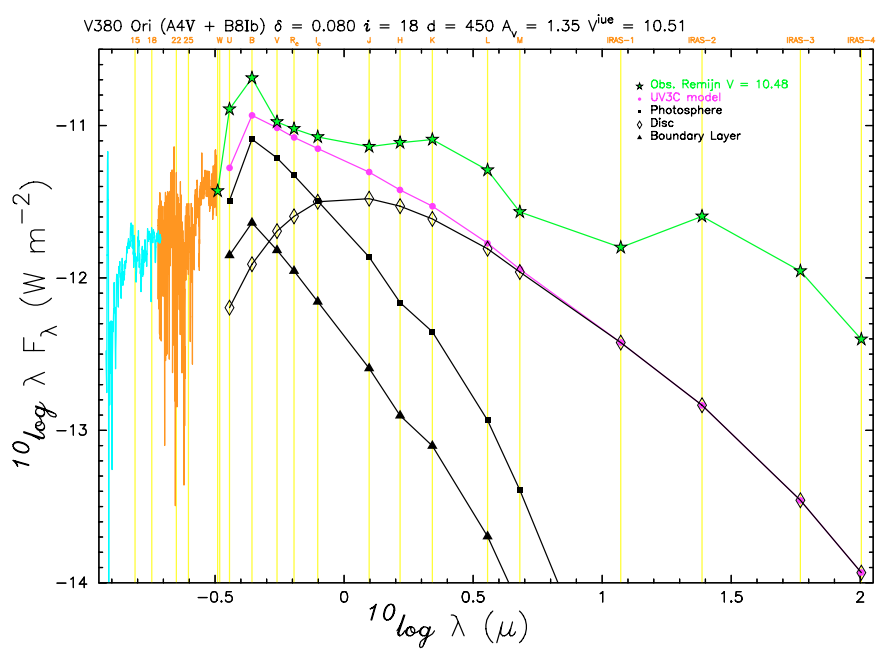

Fig. 24. V380 Ori A4 v.

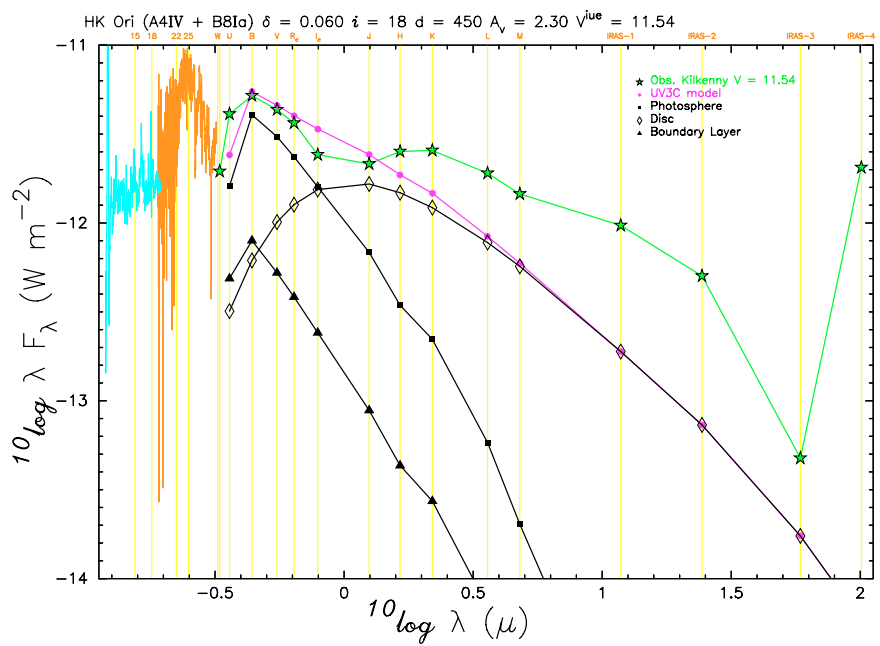

Fig. 25. HK Ori A4 IV.

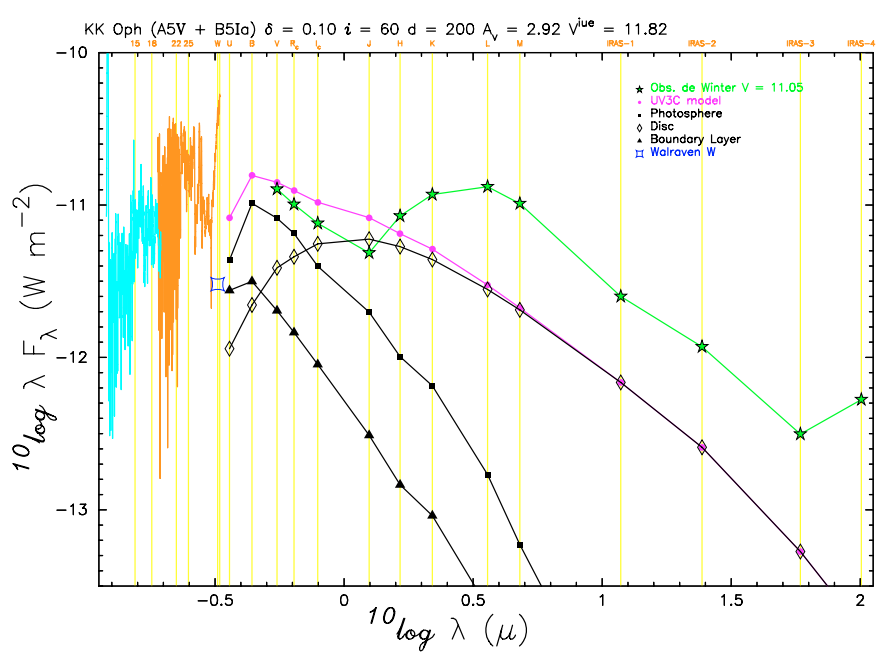

Fig. 26. KK Oph A5 v.

\subsection{The SEDs}

In the visual and near-infraredi, a large amount of photometric observations of the stars in our sample has been made and published (references of compilations: de Winter et al. 2001; 


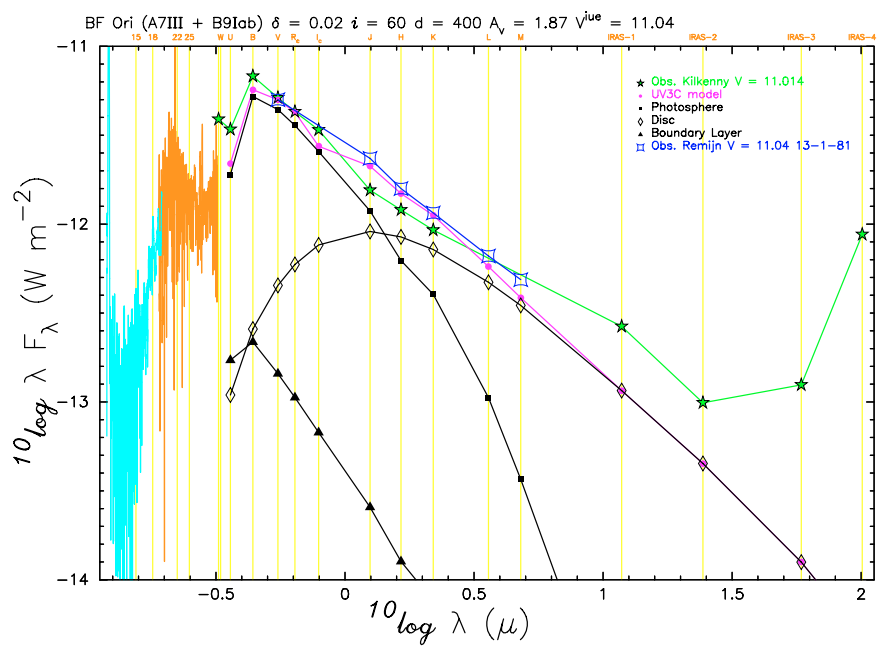

Fig. 27. BF Ori A7 III.

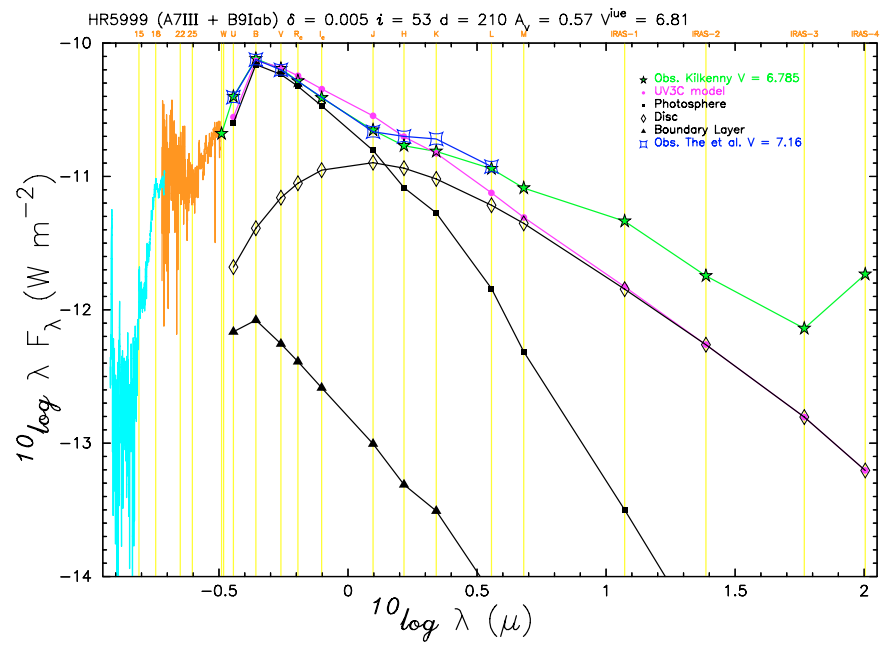

Fig. 28. HR 5999 A7 III.

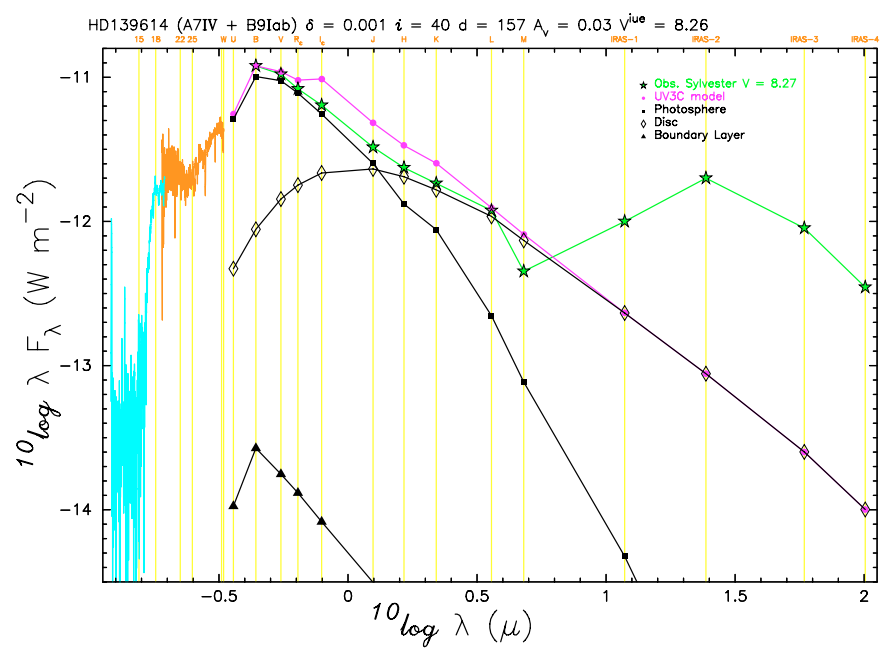

Fig. 29. HD 139614 A7 IV.

Eiroa et al. 2002; Kilkenny et al. 1985; Sylvester et al. 1996). Most of the stars are photometrically variable on time-scales of days or weeks. Only for a few stars (HD 163296, HR 5999, BF Ori, BN Ori, CQ Tau, and AK Sco) do we have simultaneous IUE and $U B V R I$ photometric observations. However, in many

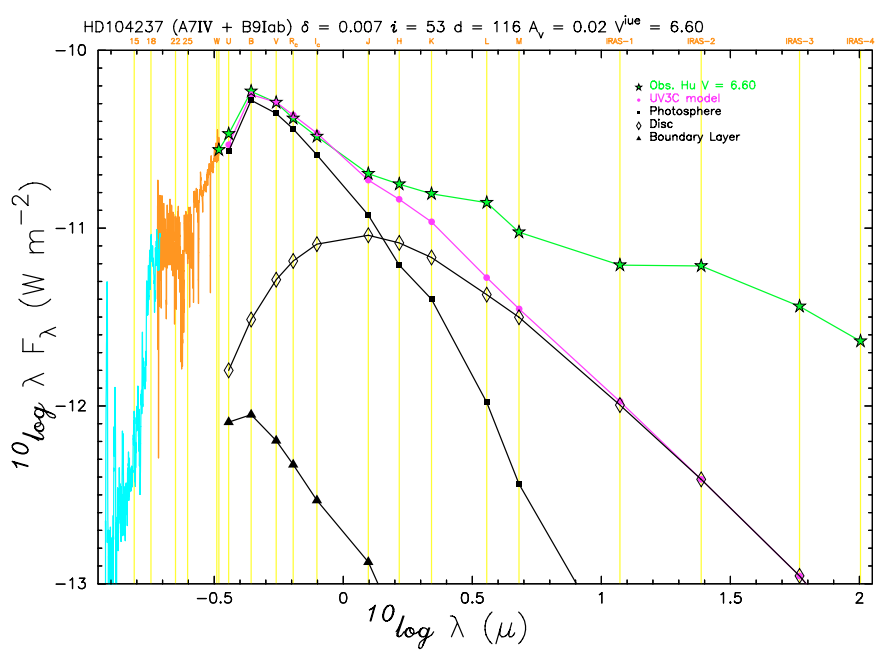

Fig. 30. HD 104237 A7 IV.

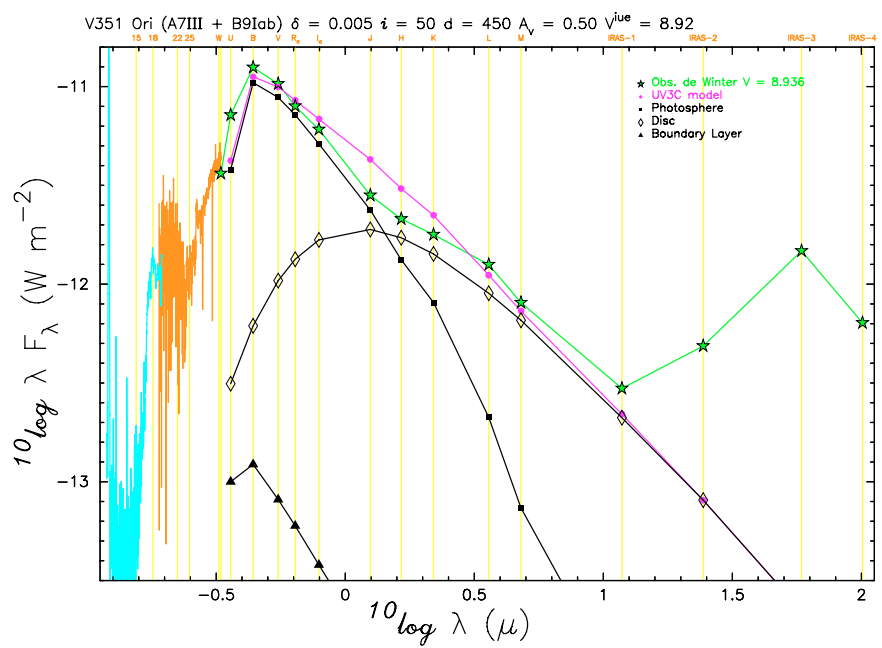

Fig. 31. V351 Ori A7 III.

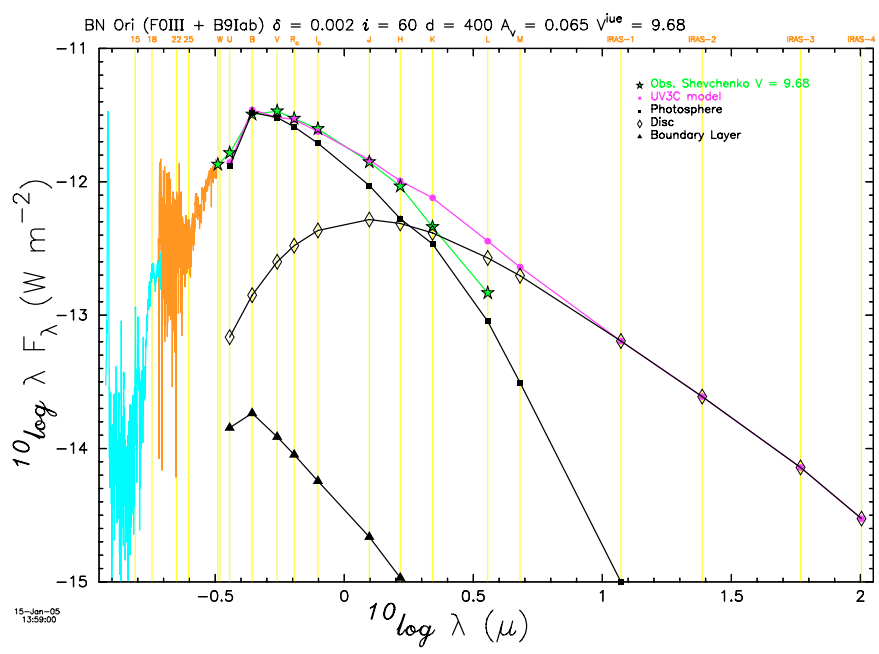

Fig. 32. BN Ori F0 III.

cases we can find Walraven WULBV, UBVRI, and JHKLM photometry in which the observed $V^{\mathrm{J}}$-magnitude is close to the $V_{\text {obs }}^{\text {iue }}$ measured with the FES during the IUE exposure of the star. In addition we found ANS data for NX Pup and HD 163296. We assumed that the IRAS PS photometry is not variable. Table 7 
Table 7. Data used for the SED-calculations, $V$-magnitude with references for each photometric observation. Observations on the $W U L B V$ (Walraven) photometric system can be found in de Winter et al. (2001) with the exception of HD 139614 and SU Aur. Observations in the 4 IRAS bands $(11.8,24.4,58.6$ and $101 \mu \mathrm{m})$ can be found in NASA RP-1190 (1988).

\begin{tabular}{lll}
\hline \hline PMS star & UBVRI & JHKLM \\
\hline HD 97048 & $8.46^{1,5,11}$ & $8.46^{1,5,11}$ \\
UX Ori & $10.91^{12}$ & $10.92^{12}$ \\
HD 163296 & $6.90^{15}, 6.88^{5}$ & $6.90^{15}, 6.88^{5}$ \\
V380 Ori & $10.48^{11}, 10.44^{10}$ & $10.48^{11}, 10.44^{11}$ \\
HK Ori & $11.52^{11}, 11.65^{10}$ & $11.63^{11}, 11.65^{10}$ \\
KK Oph & $11.82^{5}$ & $11.91^{5}$ \\
BF Ori & $11.04^{5}$ & $11.03^{5}$ \\
HR 5999 & $6.81^{10}$ & $6.80^{10}$ \\
HD 139614 & $8.26^{14}$ & $8.26^{14}$ \\
V351 Ori & $8.92^{5}$ & $8.92^{5}$ \\
HD 104237 & $6.60^{9}$ & $6.60^{9}$ \\
BN Ori & $9.68^{13}$ & $9.68^{13}$ \\
CQ Tau & $9.8^{2}, 9.45^{7}$ & $9.45^{7}$ \\
NX Pup & $10.24^{3}, 10.64^{3}$ & $10.14^{3}$ \\
AK Sco & $8.95,9.03,8.96,9.04^{10}$ & $8.95,9.02,9.04^{10}$ \\
SU Aur & $9.02^{4}, 9.09^{8}, 9.15^{12}$ & $9.02^{4}, 9.07^{8}, 9.15^{12}$ \\
\hline
\end{tabular}

1: van den Ancker (1996)

2: Berdyugin et al. (1990, 1992)

3: Brand et al. (1983)

4: Cohen \& Schwartz (1976)

5: de Winter et al. (2001)

7: Eiroa et al. (2002)

8: Eugenio \& Mendoza (1966)
HD 97048. Similar improvements of predicted SEDs are shown in Hil92-Fig. 2 for UX Ori, BF Ori, HD 150193, HR 5999, NX Pup, V380 Ori, HK Ori, KK Ori, BD $+46^{\circ} 3471$, and $\mathrm{BD}+09^{\circ} 880$ in our program.

The resulting "hole radii" may be expected to be different from those obtained by Hil92, because the photospheric spectral types adopted by them are different from our estimates in general and, as a result of this, possible existing IR excesses (e.g. in NX Pup, KK Oph, HK Ori, and V380 Ori) may be masked in their fits.

In our paper, however, we are mainly concerned with the components that dominate the UV spectrum: the photosphere and the BL. Although interesting in itself, the details of the disc model have no influence on the match to the observed UV spectrum, and will be ignored in the rest of this paper.

\subsection{UV variability}

Among the various space instruments (TD1, Copernicus, ANS, IUE, HST) for observation of the near UV (1200-3600 ^), IUE is the only one that was well-suited for repeat observations. Most of the stars in our sample have been observed at least twice and spectral changes have been noted (Valenti et al. 2000), but usually time-scales of such variability cannot be established from these data. One type of variability mentioned above may be correlated with changes in circumstellar extinction (UX Ori, BF Ori, HR 5999, CQ Tau). For this type time-scales of days or weeks have been found from observations in the visual. On smaller time-scales only the brightest PMS stars have been observed: HD $104237(V=6.60)$, HR $5999(V>6.7)$, HD 163296 $(V=6.9)$, HD $199143(V=7 \mathrm{~m}$. 1), AB Aur $(V>7$ m.2) and AK Sco $(V=8.9)$.

HD 163296 was observed with time intervals of several hours during two weeks in 1986 and 1987 (Table F.5). The conclusions from the low-resolution observations of HD 163296 are described in Sect. A.2. No periodicity was found in these data thus far. However, small variations may occur irregularly, which could be indications of low amplitude activity. Among the highresolution results, the $\mathrm{Mg}$ II-profile variations have been interpreted as due to a rotating wind structure coupled to the star, similar to the situation for AB Aur (Catala et al. 1989).

HD 163296, HD 104237, HD 199143, and HR 5999 were observed in 5 sequences of IUE observations in 1986 and 1987, 1993, 1995, and 1996 respectively. From the observed values of $v \sin i$ (Table 1), the estimated inclinations and the derived stellar radii (Table 2) we expect rotation periods of $14^{\mathrm{h}}, 21^{\mathrm{h}}$, $11^{\mathrm{h}}$, and $36^{\mathrm{h}}$, respectively. In the five sequences the observations were spread over several phases of the stellar rotations. We noted variations in the emission-line fluxes, and variations in the continuum may also be present. The results of these analyses and alternative interpretations will be discussed in Paper II.

AK Sco is a spectroscopic binary star with an accurately known $P_{\text {orb }}=13.6$ (Andersen et al. 1989). We took low and high-resolution UV spectra of successive apastron and periastron phases in $1988^{5}$. Variations in emission-line fluxes and continuum fluxes were noted, which is confirmed by visual spectroscopy (Alencar et al. 2003). The variations in the visual spectra were interpreted in terms of inhomogeneities in the common dust disc of the components. However, UV-emission flux variability may point to stellar emission variability and/or to uneven distribution of the emission regions on the surface of the binary components. (The estimated rotation period of the (equal)

4 The calculated SED of SU Aur is similar to that in BBB88.

\footnotetext{
${ }^{5}$ In collaboration with dr. A. Brown \& dr. J. L. Linsky.
} 


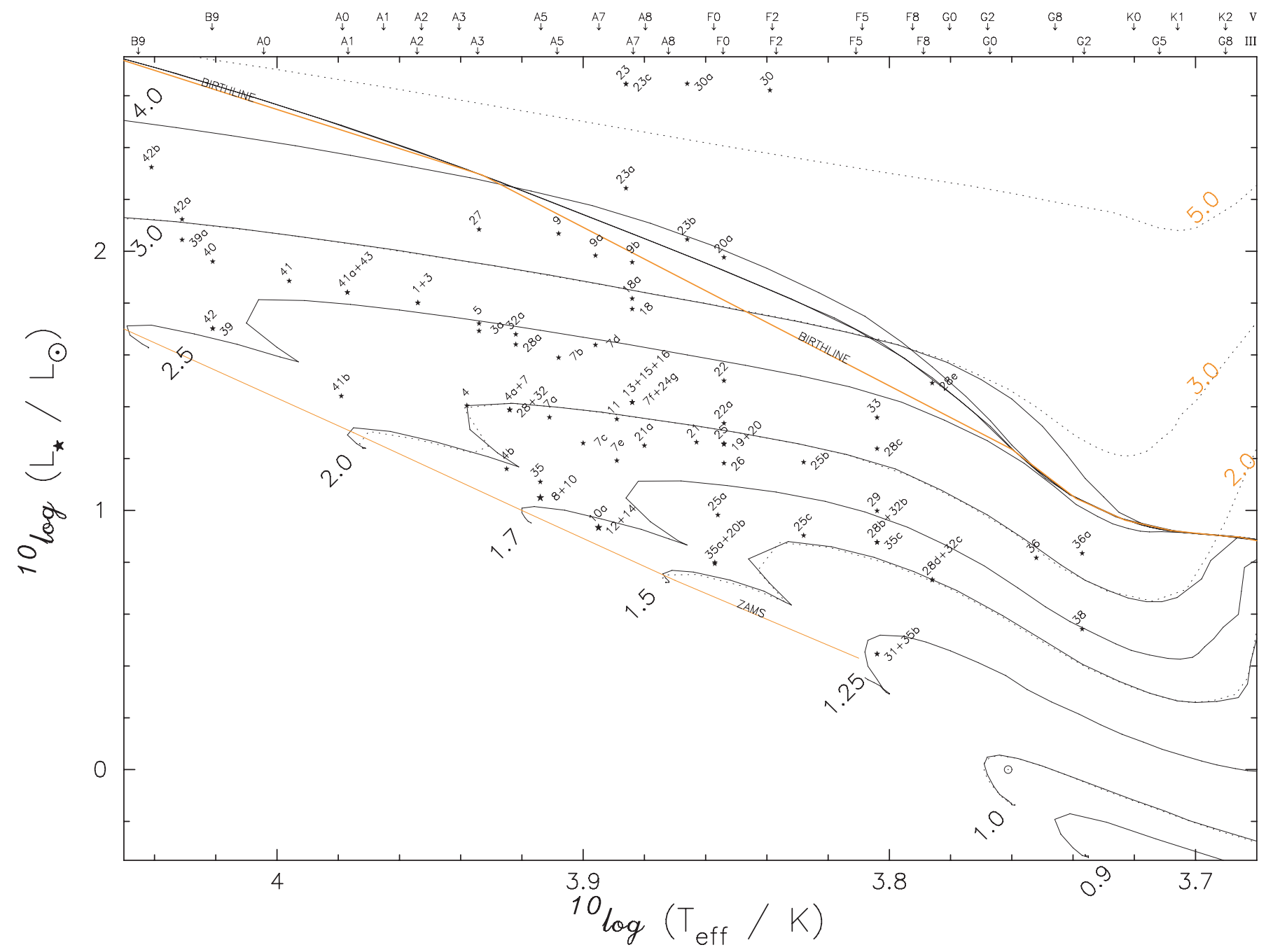

Fig. 41. HRD with - mass-labeled - (0.9-5.0) tracks of Bernasconi (1996) with the modeled stars $(\star)$, where $\odot$ is the Sun. The - upper grey line is the $10^{-5} M_{\odot} \mathrm{yr}^{-1}$ birthline with $\mathrm{Z}=0.020$ and the - lower grey - line is the ZAMS as given in Table E.1. The dotted lines are the canonical evolutionary tracks. Star-labels are from Table 2.

components is $17^{\text {h }}$, which means $\sim 11$ rotations between apastron and periastron, e.g. between the observations in April 1988: $7.778\left(=0.56 P_{\text {orb }}\right)$.) We will discuss these results in Paper II.

\subsection{2. $\delta$ Scuti pulsations}

Recently high time resolution photometry of at least 7 of the stars in our sample: HR 5999, $\beta$ Pic, HD 104237, HD 142666 , V346 Ori, V351 Ori, and HD 35929, have revealed that these stars show $\delta$ Scuti-type pulsations. With the stellar parameters in Table 2, we can determine the location of the stars in the HRD and also derive the instability modes corresponding to the observed pulsation periods. The results will be given and discussed in a research note.

\subsection{Contribution by UV emission lines}

In the SW-range of many spectra we observe the presence of emission-lines of O I, C II, Si II, Si III, Si IV, C II, and C IV (Tjin A Djie et al. 1998). In the LW-range we often observe emission-lines of Mg II and Fe II. We shall discuss the emission fluxes of these lines in a separate paper (Paper II).

\section{Mass accretion rates}

The UV3C analysis of each low-resolution UV spectrum of a star with known $d$ and $\boldsymbol{E}^{\text {is }}$ gives us (for chosen ST and LC of stellar photosphere and BL) the $\delta_{\mathrm{bl}}$ and the circumstellar extinction parameters $A_{\mathrm{v}}^{\mathrm{cs}}$ and $R^{\mathrm{cs}}$. Fortunately the value of the distance strongly affects the UV3C model spectrum, so that $d$ can usually be constrained within $\pm 15 \%$. From the distance and the observed $V^{\mathrm{J}}$-flux (corrected for foreground extinction), we obtained in each case a value for the photospheric radius; and with the additional knowledge of $T_{\text {eff }}$ (from adjusting ST and LC), we calculated $L_{\text {bol }}$, so that we can locate the star in the HRD (Fig. 41). With the help of the PMS evolutionary tracks for models of $0.5-7 M_{\odot}$ stars, calculated by PS93, Bernasconi (1996), and Siess et al. (1999, 2000), we then interpolated values for $M_{\star}$ and $t_{\star}$. The results of the interpolation of the Bernasconi tracks are given in Tables 2 and 6 .

The mass accretion rates $(\dot{M})$ were derived from the energy balance between the viscous energy produced in the disc and $\mathrm{BL}$ and the energy radiated by the BL (Eq. (5)), where the stellar influx on the inner layers of the disc (less than a few percent) has been neglected. Here we approximate the BL flux by that of the Planck spectrum of the same $T_{\text {eff }}$ as listed for the 
supergiant spectral type B8 I or B9 I (SK82). For the integrated spectral flux, this approximation is correct within a few percent. In the case of a co-rotating star, part of the viscous energy is used to spin-up the star (see e.g. Kley \& Lin 1996), so that the energy available for BL radiation becomes less. In order to observe the same radiation flux from the $\mathrm{BL}$ as in the non-rotating situation (as calculated by the UV3C-model), we have to raise the viscous dissipation, i.e. the accretion rate. However, this correction could only be made by us for objects for which the $v \sin i$-value and the inclination angle $i$ are known (Table 1). Then for a radially thin BL the integrated flux can be obtained from:

$\frac{G M_{\star} \dot{M}^{\mathrm{c}}}{2}\left(1-\frac{\Omega_{\star}}{\Omega_{\mathrm{K}}}\right)^{2}=4 \pi R_{\star}^{2} \delta \sigma T_{\mathrm{bl}}^{4}$

where $\quad \Omega_{\star}=\frac{v}{R_{\star}} \quad$ and $\quad \Omega_{\mathrm{K}}=\sqrt{\frac{G M_{\star}}{R_{\star}^{3}}}$.

The mass accretion rates $\left(\dot{M}\right.$ and $\left.\dot{M}^{\mathrm{c}}\right)$ derived from Eqs. (5) and (14) are listed in Tables 2 and 6.

\subsection{Relation between $\dot{M}^{c}$ and $M_{\star}$}

Several authors (see Muzerolle et al. 2005) have reviewed the accretion rates for $\mathrm{T}$ Tauri stars and brown dwarfs, which results in $\dot{M}^{\mathrm{c}} \propto M_{\star}^{2}$. Figure 42 shows that our data for more massive stars roughly follow the same relation, although the $\dot{M}^{\mathrm{c}}$ is determined in a different way.

Recently Padoan et al. (2005) re-examined the source of accretion on young stars. It has been realised that without influx the circumstellar discs of PMS-stars do not contain sufficient mass to support the observed mass-accretion rate by self-gravity or turbulence for more than a few Myr. However, PMS-stars can in general be associated with the molecular cloud complexes (in particular dense filaments) in which they were born. It seems natural to assume that a few Myr after the disc formation, the parent cloud will become the main supply of accretion. In this case the accretion rate will only depend on the stellar mass and the conditions in the cloud. Padoan et al. used the Bondi-Hoyle (1944) fluid dynamical accretion rate for a mass $M_{\star}$ moving supersonically with velocity $v$ through a uniform cloud medium of density $\rho$ as an estimate of the accretion on the star-disc system:

$\dot{M}=\frac{4 \pi G^{2} \rho}{\left(c^{2}+v^{2}\right)^{\frac{3}{2}}} \quad M_{\star}^{2}=K \quad M_{\star}^{2}$

where $\rho, c$, and $v$ are gas density, sound speed, and relative velocity of the gas averaged over some distance (e.g. 500 AU) in the large-scale cloud. Numerical simulations of star formation in self-gravitating turbulent clouds have supplied values for the three quantities and enabled the authors to estimate the coefficient $K$ from Eq. (16). For $10^{4}>\rho>10^{3} \mathrm{~cm}^{-3}$, they find $K=10^{-7.6 \pm 0.7}$ (Fig. 42 dashed lines), while for $\rho>10^{4} \mathrm{~cm}^{-3}$ the result is $K=10^{-6.6 \pm 0.6}$ (Fig. 42 solid lines). These $K$-values give a good account of the accretion rates that are found in PMS objects of various masses. Even accretion rates of $10^{-11} M_{\odot} \mathrm{yr}^{-1}$ are correctly predicted for brown dwarfs of $0.03 M_{\odot}$ if $K \sim$ $10^{-8}$. Figure 42 may be compared with Muzerolle et al. (2005) Fig. 5. The young PMS Be/Ae/Fe stars analysed in this paper have masses of $1.8-4.0 M_{\odot}$. A group of 14 stars is situated above the $K=10^{-7.2}$ line and 11 below. We note that 6 out of these 14 are associated with dark clouds (Appendix C), while the second group of stars has only associations with well-known regions

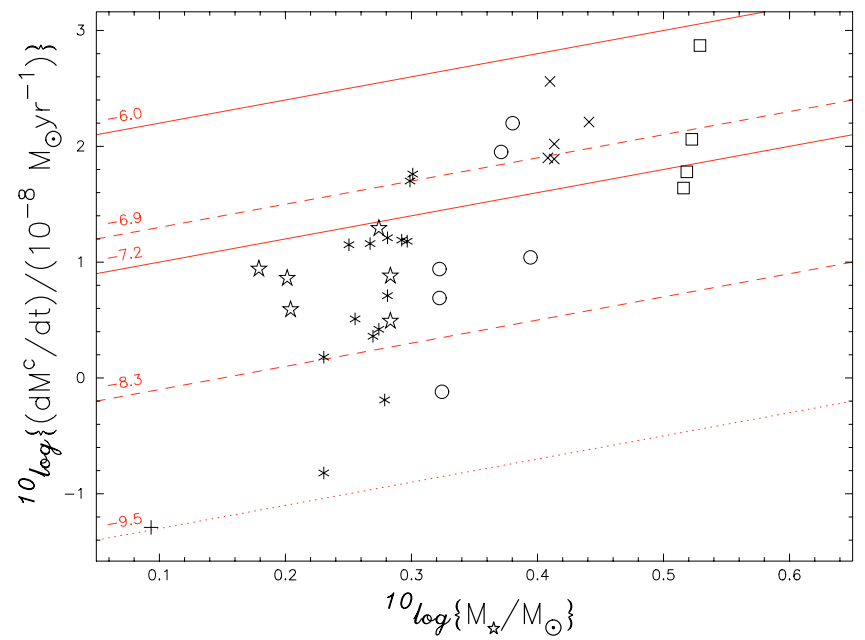

Fig. 42. Corrected mass-accretion rate versus stellar mass. The solid and dashed lines are from Padoan et al. (2005). Numbers indicate the ${ }^{10} \log K$-values and the dotted line is the detection limit. See Fig. 44 for the mass-symbols.

of star formation. Therefore, this second group follow the same trend as the T Tauri stars and seem to be associated with clouds of similar densities, but higher than those of brown dwarfs. We conclude that the accretion rates that we measured for the $\mathrm{PMS} \mathrm{Be/Ae/Fe} \mathrm{stars} \mathrm{are} \mathrm{an} \mathrm{extrapolation} \mathrm{of} \mathrm{the} \mathrm{accretion} \mathrm{rates}$ obtained for T Tauri stars. We note, however, that HD 163296 belongs to the "high rate group" in spite of the fact that it seems to be an "isolated" star (e.g. no association with clouds). It is also not clear if the high rates for FU Ori and Z CMa are constant rates in time (not related to the outbursts of these objects). It could mean that they are associated with particularly dense parent cloud filaments. The molecular parent cloud connection gives a good overall account of the mass dependence of the derived accretion rates in terms of assumed differences in stellar mass and gas densities of the associated filaments in the parent cloud. However, the model is a static one and does not account for the further evolution of the accretion in the star-disc systems of various mass. It also ignores the role of the disc replenishment process played by the nearby material, ejected in (bipolar) outflow by the star. We shall now briefly discuss these points.

\subsection{Evolutionary trends}

Since the mass values derived from the models of Bernasconi (1996) and Siess et al. (2000) do not differ very much (Table 8) and the rates are not very sensitive to the mass values, the rates from the various models are very similar. In order to search for evolutionary trends in the rates we have to determine the ages of the stars $\left(t_{\star}\right)$ from the calculated models. In contrast to the masses, the ages derived from the three models, are in general very different. This is mainly because small differences in mass from the various models lead to large differences in age. We expect higher ages from the models of Siess that include accretion, because these models start with lower mass and therefore evolve more slowly than the models with fixed mass. Although this seems to be true if we compare the ages resulting from the accretion-included models of Siess et al. (1999) and the fixedmass models of Bernasconi, it is less clear and often reversed if we compare the accretion-included models of Siess et al. (1999) with the fixed-mass models of Siess et al. (2000). We find, however, that the differences in relative age (absolute age divided by 


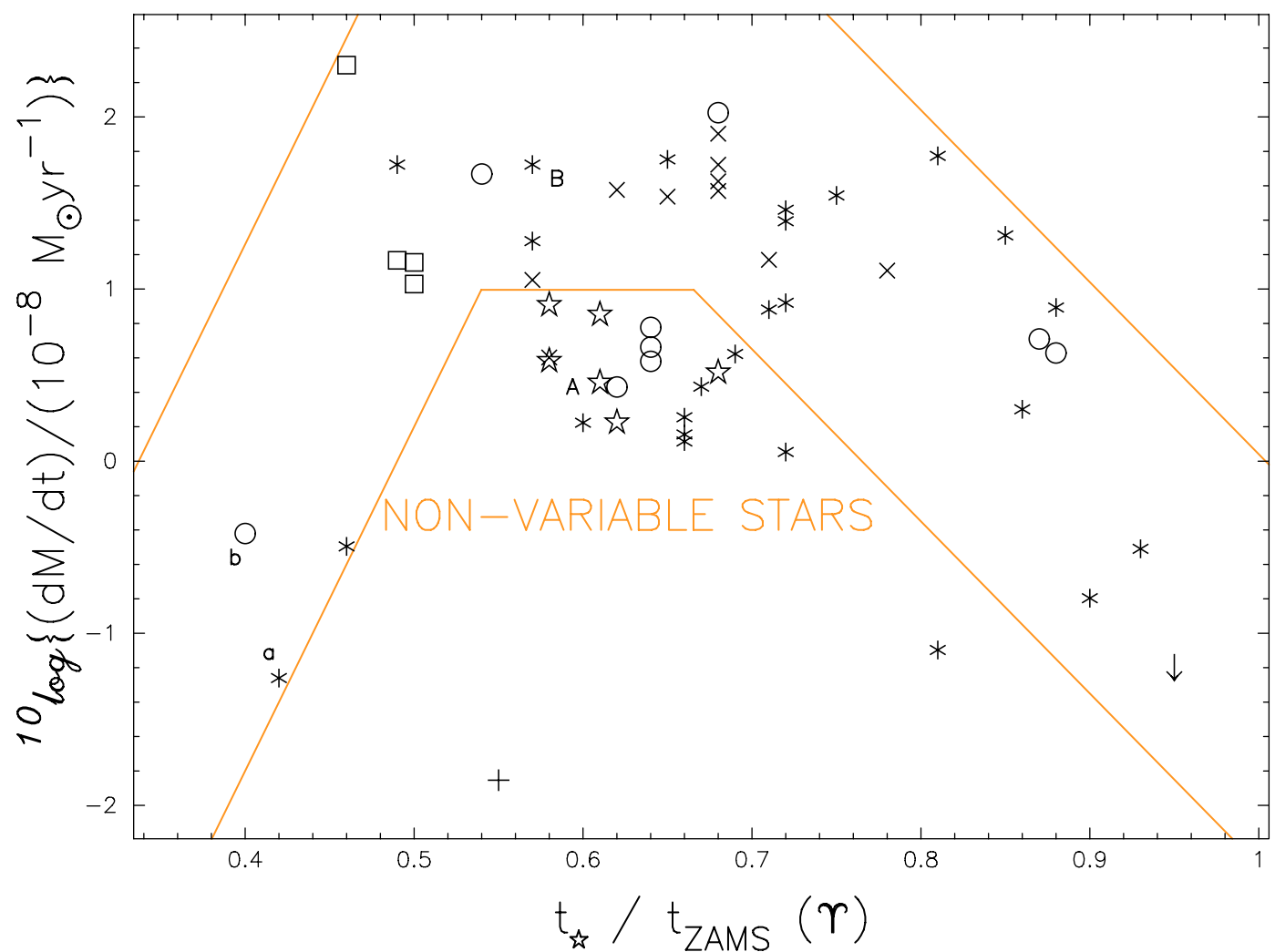

Fig. 43. Mass-accretion rate (Table 2) versus relative age (Table 8). The arrow indicates the age of $\beta$ Pic. See Fig. 44 for the mass-symbols.

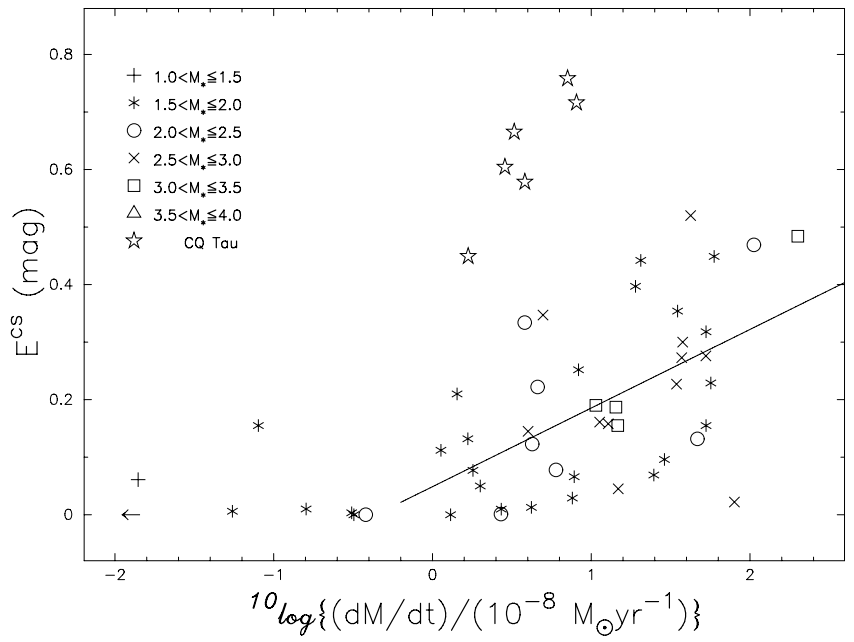

Fig. 44. Mass-accretion versus circumstellar extinction (Table 2), with a linear-least-square line of the points with $\dot{M}>10^{-8} M_{\odot} \mathrm{yr}^{-1}$ and without CQ Tau. The arrow indicates the $\boldsymbol{E}^{\mathrm{cs}}$-value of $\beta$ Pic.

ZAMS age (Appendix E) for the same mass): $\Upsilon=t_{\star} / t_{\text {ZAMS }}$ are much smaller.

In Table 8 we compare the masses and $\Upsilon$ resulting from the interpolation of the 3 sets of tracks. In the latter case accretion during the evolution is taken into account. The mass accretion rate was assumed to decrease exponentially after 4.5 Myr. It should be noted that the relative ages obtained with the Case-B grid are not systematically different from those obtained with the other 2 grids, although in Case-B the initial mass is lower and the initial evolution slower than in the other models.

Figure 43 shows the distribution of the accretion rates (derived above from the UV spectra) with the relative age of the stars in our sample. We note that the higher rates occur for $0.45<\Upsilon<0.80$ and that outside this range we only find lower rates. Rates of $<10^{-7} M_{\odot} \mathrm{yr}^{-1}$, which occur for $0.55<\Upsilon<0.70$, appear to correspond to a group of non-variable stars, among which are the formerly variable stars BN Ori and V351 Ori. The stars in the trapezium shaped region labelled non-variable stars mainly contains stars that are not photometrically variable (details in Tables 2 and 8). CQ Tau also appears in this region, presumably because its rate is derived from spectra obtained at high brightness, in which the accretion activity is low (Kozlova et al. 2000). Figure 43 suggests that:

(1) during the approach to the ZAMS $\dot{M} \propto 10^{-10 \Upsilon}$.

Although the decrease is to be expected, the mechanism for this behaviour is not yet clear; and

(2) during the early evolution $\dot{M} \propto 10^{20 \Upsilon}$.

If this is true, the young $\left(t_{\star} \sim 2.25 \mathrm{Myr}, \Upsilon=0.39\right) 2 M_{\odot}$ star, SU Auri, can be the precursor of a $2.4 M_{\odot}$ star (with $t_{\star} \sim$ $3.25 \mathrm{Myr}, \Upsilon=0.58$ ), if the average mass accretion rate becomes $4 \times 10^{-7} M_{\odot} \mathrm{yr}^{-1}$ and if there is negligible mass loss from the sytem during this phase $(\mathrm{b} \rightarrow \mathrm{B}$, Fig. 43). In the same way the $1.7 M_{\odot}$ star HD 283572 (with $t_{\star} \sim 6.7 \mathrm{Myr}, \Upsilon=0.41$ ) can become a $1.77 M_{\odot}$ star with $t_{\star}=10 \mathrm{Myr}(\Upsilon=0.60)$, if its present rate of $\sim 10^{-9} M_{\odot} \mathrm{yr}^{-1}$ is increasing by a factor $20(\mathrm{a} \rightarrow \mathrm{A}$, Fig. 43).

\subsection{Circumstellar extinction}

The other products of the UV3C analysis are $A_{\mathrm{v}}^{\mathrm{cs}}$ and $R^{\mathrm{cs}}$, or $\boldsymbol{E}^{\mathrm{cs}}$ (Table 2). The relation between $\boldsymbol{E}^{\mathrm{cs}}$ and $\dot{M}$ (Fig. 44) is not completely clear. The figure may suggest that significant $\boldsymbol{E}^{\text {cs }}$ dust excesses only occur for sufficiently high accretion rates as expected for bipolar outflow during accretion. However, the number of 
Table 8. Comparison of the determined stellar masses $\left(M_{\odot}\right)$ and relative ages, spectral type $z$ indicates ZAMS.

\begin{tabular}{|c|c|c|c|c|c|c|c|c|}
\hline \multirow[b]{2}{*}{ PMS star } & & \multirow{2}{*}{$\begin{array}{l}\text { spec. } \\
\text { type }\end{array}$} & \multicolumn{2}{|c|}{ Bernasconi } & \multicolumn{2}{|c|}{ "Siess-NG } & \multicolumn{2}{|c|}{$\overline{\overline{\text { Siess-B }}}$} \\
\hline & & & $M_{\star}$ & $\Upsilon$ & $M_{\star}$ & $\Upsilon$ & $M_{\star}$ & $\Upsilon$ \\
\hline XY Per & & A2 III & 2.59 & 0.62 & 2.60 & 0.60 & 2.61 & 0.67 \\
\hline UX Ori & & A2 III & 2.59 & 0.68 & 2.60 & 0.64 & 2.61 & 0.68 \\
\hline UX Ori & $\mathrm{a}$ & A3 III & 2.40 & 0.68 & 2.43 & 0.64 & 2.42 & 0.65 \\
\hline HD 163296 & & A3 IV & 2.00 & 0.72 & 2.17 & 0.81 & 2.05 & 0.76 \\
\hline HD 163296 & $\mathrm{a}$ & A4 IV & 1.99 & 0.72 & 2.00 & 0.83 & 2.02 & 0.71 \\
\hline HD 150193 & & A3 III & 2.56 & 0.68 & 2.60 & 0.64 & 2.57 & 0.66 \\
\hline BF Ori & $\mathrm{c}$ & A6 IV & 1.85 & 0.88 & 1.88 & 0.86 & 1.85 & 0.80 \\
\hline BF Ori & $\mathrm{e}$ & A7 IV & 1.78 & 0.71 & 1.87 & 0.70 & 1.80 & 0.57 \\
\hline HD 31648 & & $\mathrm{~A} 5 \mathrm{Vz}$ & 1.80 & 0.86 & 1.83 & 0.90 & 1.80 & 0.83 \\
\hline HR 5999 & & A5 III & 3.50 & 0.50 & 3.44 & 0.56 & 3.36 & 0.59 \\
\hline HR 5999 & $\mathrm{a}$ & A6 III & 3.30 & 0.50 & 3.25 & 0.55 & 3.23 & 0.59 \\
\hline HR 5999 & $\mathrm{~b}$ & A7 III & 3.33 & 0.49 & 3.30 & 0.55 & 3.23 & 0.57 \\
\hline$\beta$ Pic & $\mathrm{a}$ & A7 Vz & 1.65 & 0.95 & 1.72 & 1.00 & 1.68 & 1.00 \\
\hline HD 104237 & & A7 IV & 1.96 & 0.69 & 2.00 & 0.65 & 1.97 & 0.63 \\
\hline HD 104237 & & A7 IV & 2.17 & 0.67 & 2.14 & 0.64 & 2.15 & 0.62 \\
\hline HD 169142 & & A7 Vz & 1.65 & 0.93 & 1.76 & 0.91 & 1.79 & 0.73 \\
\hline HD 36112 & & A7 III & 2.10 & 0.64 & 2.13 & 0.62 & 2.09 & 0.60 \\
\hline HD 139614 & & A7 Vz & 1.65 & 0.90 & 1.72 & 1.00 & 1.68 & 1.00 \\
\hline HD 142666 & & A7 III & 2.10 & 0.64 & 2.13 & 0.62 & 2.09 & 0.60 \\
\hline V346 Ori & & A7 III & 2.10 & 0.64 & 2.13 & 0.62 & 2.09 & 0.60 \\
\hline V351 Ori & & A7 III & 2.80 & 0.57 & 2.78 & 0.57 & 2.80 & 0.63 \\
\hline V351 Ori & $\mathrm{a}$ & A7 III & 2.91 & 0.58 & 2.89 & 0.57 & 2.88 & 0.63 \\
\hline BN Ori & & F0 III & 1.91 & 0.66 & 1.94 & 0.61 & 1.92 & 0.58 \\
\hline HD 35929 & & F0 III & 1.91 & 0.66 & 1.94 & 0.61 & 1.92 & 0.58 \\
\hline HD 35929 & $\mathrm{~b}$ & F0 V & 1.62 & 0.81 & 1.61 & 1.0: & 1.60 & 0.74 \\
\hline HD 144432 & & F0 III & 1.91 & 0.66 & 1.94 & 0.61 & 1.92 & 0.58 \\
\hline HD 144432 & & F0 V & 1.61 & 0.72 & 1.61 & 1.0: & 1.60 & 0.74 \\
\hline $\mathrm{BD}+47^{\circ} 4206$ & & F0 III & 2.34 & 0.62 & 2.35 & 0.60 & 2.33 & 0.60 \\
\hline CQ Tau & & F0 III & 1.92 & 0.61 & 1.94 & 0.62 & 1.92 & 0.58 \\
\hline CQ Tau & $\mathrm{a}$ & F0 IV & 1.59 & 0.68 & 1.68 & 0.57 & 1.68 & 0.52 \\
\hline CQ Tau & $\mathrm{b}$ & F3 III & 1.88 & 0.58 & 1.90 & 0.58 & 1.92 & 0.53 \\
\hline CQ Tau & $\mathrm{c}$ & F3 IV & 1.51 & 0.58 & 1.61 & 0.56 & 1.56 & 0.58 \\
\hline CQ Tau & $\mathrm{d}$ & F0 III & 1.92 & 0.61 & 1.94 & 0.62 & 1.92 & 0.58 \\
\hline CQ Tau & $\mathrm{e}$ & F2 IV & 1.60 & 0.62 & 1.65 & 0.60 & 1.62 & 0.54 \\
\hline NX Pup & & F2 III & 1.86 & 0.65 & 1.86 & 0.56 & 1.87 & 0.55 \\
\hline $\mathrm{BD}+46^{\circ} 3471$ & & A3 III & 3.38 & 0.46 & 3.33 & 0.56 & 3.29 & 0.62 \\
\hline HK Ori & & A4 IV & 1.97 & 0.75 & 2.00 & 0.81 & 2.02 & 0.71 \\
\hline HK Ori & & F6 V & 1.24 & 0.49 & 1.35 & 0.45 & 1.28 & 0.74 \\
\hline AK Sco & & F6 IV & 1.70 & 0.60 & 1.76 & 0.57 & 1.73 & 0.50 \\
\hline HD 199143 & & F6 V & 1.25 & 0.55 & 1.40 & 0.76 & 1.30 & 0.57 \\
\hline V380 Ori & $\mathrm{a}$ & A4 IV & 1.99 & 0.75 & 2.00 & 0.82 & 2.02 & 0.71 \\
\hline V380 Ori & $\mathrm{b}$ & F6 IV & 1.58 & 0.57 & 1.60 & 0.55 & 1.59 & 0.45 \\
\hline NV Ori & & F6 III & 2.35 & 0.54 & 2.32 & 0.53 & 2.32 & 0.55 \\
\hline KK Oph & & A5 V & 1.86 & 0.81 & 1.85 & 0.81 & 1.80 & 0.80 \\
\hline KK Oph & $\mathrm{a}$ & F0 V & 1.61 & 0.85 & 1.60 & 0.91 & 1.58 & 0.71 \\
\hline KK Oph & $\mathrm{c}$ & F6 IV & 1.58 & 0.57 & 1.58 & 0.55 & 1.50 & 0.50 \\
\hline SU Aur & & G2 IV & 1.90 & 0.46 & 1.80 & 0.48 & 1.83 & 0.43 \\
\hline SU Aur & $\mathrm{a}$ & G5 IV & 2.14 & 0.40 & 2.00 & 0.45 & 2.00 & 0.39 \\
\hline HD 283572 & & G5 IV & 1.70 & 0.42 & 1.60 & 0.44 & 1.63 & 0.36 \\
\hline $\mathrm{BD}+09^{\circ} 880$ & & B9Vz & 2.56 & 0.87 & 2.62 & 0.84 & 2.60 & 0.86 \\
\hline HD 100546 & & B9 V & 2.70 & 0.65 & 2.83 & 0.64 & 2.74 & 0.70 \\
\hline HD 98922 & & $\mathrm{~A} 0 \mathrm{Vz}$ & 2.19 & 0.88 & 2.20 & 0.85 & 2.24 & 0.86 \\
\hline HD 98922 & $\mathrm{a}$ & A0IV & 2.63 & 0.78 & 2.67 & 0.66 & 2.63 & 0.71 \\
\hline HD 98922 & $\mathrm{~b}$ & A1 III & 2.57 & 0.71 & 2.61 & 0.65 & 2.59 & 0.68 \\
\hline HD 97048 & & B9 Vz & 2.48 & 0.84 & 2.63 & 0.84 & 2.60 & 0.86 \\
\hline HD 95881 & & A1 III & 2.57 & 0.68 & 2.70 & 0.62 & 2.67 & 0.69 \\
\hline
\end{tabular}

stars in our sample with a low accretion rate is too low to be statistically significant, which does not permit us to draw a definite conclusion. In addition, for the points with $\dot{M}>10^{-8} M_{\odot} \mathrm{yr}^{-1}$, the $\boldsymbol{E}^{\mathrm{cs}}$ is correlated neither with accretion rate (as suggested by the dispersion around the linear least-square line in the figure) nor with the stellar mass.
Table 8. continued.

\begin{tabular}{llllllll}
\hline \hline \multirow{3}{*}{ PMS star } & spec. & \multicolumn{2}{c}{ Bernasconi } & \multicolumn{3}{c}{ Siess-NG } & \multicolumn{2}{c}{ Siess-B } \\
& type & $M_{\star}$ & $\Upsilon$ & $M_{\star}$ & $\Upsilon$ & $M_{\star}$ & $\Upsilon$ \\
\hline XY Per & p, s1 & 2.59 & 0.68 & 2.60 & 0.64 & 2.61 & 0.67 \\
& s2 & 2.43 & 0.68 & 2.43 & 0.64 & 2.42 & 0.67 \\
& s3 & 2.33 & 0.64 & 2.34 & 0.63 & 2.28 & 0.64 \\
NX Pup & p, s1 & 1.86 & 0.65 & 1.86 & 0.56 & 1.87 & 0.55 \\
& s2 & 1.89 & 0.40 & 1.75 & 0.45 & 1.84 & 0.38 \\
& p, s1 & 1.86 & 0.65 & 1.86 & 0.56 & 1.87 & 0.55 \\
& $\mathrm{~s} 2$ & 1.97 & 0.37 & 1.85 & 0.45 & 1.93 & 0.39 \\
BD+46 3471 & p1 & 2.67 & 0.54 & 2.67 & 0.53 & 2.65 & 0.61 \\
& $\mathrm{~s} 1$ & 2.73 & 0.54 & 2.73 & 0.53 & 2.70 & 0.61 \\
& $\mathrm{~s} 2$ & 2.86 & 0.46 & 2.79 & 0.50 & 2.84 & 0.54 \\
AK Sco & -all- & 1.43 & 0.57 & 1.46 & 0.56 & 1.43 & 0.46 \\
KK Oph & p1 & 1.86 & 0.70 & 1.86 & 0.86 & 1.80 & 0.80 \\
& $\mathrm{~s} 1, \mathrm{p} 2$ & 1.58 & 0.57 & 1.60 & 0.55 & 1.50 & 0.50 \\
& $\mathrm{~s} 2$ & 1.70 & 0.40 & 1.74 & 0.44 & $1.82:$ & 0.27 \\
\hline
\end{tabular}

\section{Summary}

With the IUE a large collection of PMS Ae/Fe stars has been observed. This invites us to look for common properties and for variation of properties with spectral type and visual brightness. For this purpose we decided to analyse the low-resolution UV spectra in terms of a simple model with 3 components: a photosphere, an accretion disc, and a boundary-layer (BL) between star and $\operatorname{disc}^{6}$. As a result we often find spectral types that are different from the types quoted in the literature (as e.g. used by Valenti et al. 2000), which are generally derived from visual spectroscopy.

Some of the classification tools in the visual may not be reliable for estimating the spectral type of PMS Ae/Fe stars. In particular the presence of He I absorption lines at $5876 \AA$ and $6678 \AA$ has often been used as an indicator that a star has a hot photosphere and that the star should be classified as B9 or earlier. However, we find these absorption lines in the spectra of many Herbig stars, which we classify from their UV continuum as types A2e up to F8. Examples are WW Vul, UX Ori (A2-3e), BF Ori (A4-7e), CQ Tau (F2e) (Grinin et al. 2001), HD 150193 (A4e) (Catala et al. 1986), HR 5999 (A7e), NX Pup (F2e), NV Ori (F6e) (Böhm \& Catala 1995), HD 142666 (A8e) (Dunkin et al. 1997b), HD 163296 (A3-4e), HD 104237 (A7e) (Morrison 1997), HD 36112 (A7e) (Beskrovnaya et al. 1999), R CrA (A5-F2e) (Graham 1992), AK Sco (F5e), CO Ori (F8e) (de Winter 1998), and SU Aur (G2-5e) (Petrov 1996). In many cases the strength of the He I lines is highly variable on time scales of days and in such cases the absorption component sometimes disappears completely (e.g. RR Tau and CQ Tau, Kozlova et al. 2000; Grinin et al. 2001). Because of the high excitation energy, the large width, and the variability of the lines we suspect that they are formed in a hot part of the accretion disc or its turbulent atmosphere. In cases where the $\mathrm{He}$ I lines are in emission they could be formed in a chromosphere around the star for spectral types later than F2, and for earlier spectral types in a layer around the accretion disc.

For certain Herbig stars there may be evidence of accretion guided by magnetic channelling from the disc (e.g. HD 104237), but so far such cases are rare in comparison with the many cases

6 Although for the A and F stars the direct flux contribution from the disc is negligible in the wavelength range of IUE, for $i<90^{\circ}$ the shadow of the disc on the stellar surface affects the result of the calculated spectrum, hence the name "UV3C" for our analyses (also there is no BL possible without a disc). 
of evidence for such processes in T Tauri stars. In the simple UV3C model the emission from nearby dust is also ignored. We simulated the UV spectra of the stellar and BL components by low-resolution UV spectra of standard stars and that of the disc by a Planck spectrum. The BL spectral types B8 I and B9 I have effective temperatures that agree with those predicted by the theoretical 1-D BL models. After adoption of a value for the distance and the foreground colour excess from the literature and the disc inclination from various arguments, we matched the low-resolution UV spectral flux between 1200 and $3200 \AA$ for each star together with the flux in the $V^{\mathrm{J}}$-band, by varying the spectral and luminosity types of photosphere and BL, the width of the BL, and the ratio of total to specific absorption for circumstellar dust $R^{\mathrm{cs}}$. From the UV3C-model parameters we obtained values for radius and luminosity of the photosphere (which locates the star in the HRD), the circumstellar excess, and values of the width and temperature of the BL, all of which allowed us to estimate the accretion rate. In the case of double stars the brightness ratio of the components gives an additional constraint. Two kinds of comparisons have been made:

\section{[A] Time variation of individual stars}

(1) HD 163296, HD 104237, HD 199143, HR 5999, and AK Sco were observed with IUE during several days with time intervals of several hours. From the rotation rates we can derive the succesive parts of the surface we are observing during each exposure. Small variations in continuum flux and in emission-line fluxes were noted. Only HD 163296 (Sects. 5.11 and A.2) and AK Sco (Sects. 5.9 and 5.11) have been discussed shortly here.

(2) for UX Ori, BF Ori, HR 5999, and CQ Tau we have spectra at various phases of visual brightness, but separated in time by months or years. The parameter that mainly varies is $R^{\mathrm{cs}}$, but for fainter phases of the stars the agreement between model spectrum and observed spectrum becomes less satisfactory in the wavelength range 1800-2300 $\AA$. This suggests that the assumed c.s. dust extinction laws are not adequate for increasing obscuration of the stars.

\section{[B] Evolutionary trends}

We have obtained the positions of the stars in the HRD. From the evolutionary calculations of Palla, Bernasconi, and Siess we estimated values for the mass and age of each star. Table 8 shows that the differences between the results of the estimates from the models of Bernasconi and Siess are in general small. On the other hand, the spectral matching also provides us with the accretion rate and the c.s. dust colour excess of each star. The rates appear to have the same dependence on mass as T Tauri stars. This may suggest that these rates have the same origin; i.e. they are controlled by the conditions in the molecular clouds in which they were formed. However, we expect that the intrinsic evolution of the star-disc systems will have a significant influence on the presently obtained accretion rates. Figure 43 suggests an increase in the accretion rate during early phases of the contraction towards the MS and a decrease during the approach to the ZAMS. It is curious that the non-variable stars seem to have lower accretion rates than the variable stars of the same relative age between 0.5 and 0.7 .

Finally we applied the UV3C parameters to predict the SEDs of the stars in the visual and NIR. For B- and A-type stars the flux contribution of the BL is only significant in the $U$ and $B$ photometric bands. The relative flux contribution of the disc increases with increasing wavelength. In several cases the observed fluxes show an NIR excess with respect to the calculated model fluxes, which can be interpreted by the presence of dust in the neighbourhood of the star. There are also a number of cases where the contribution of the disc seems larger than observed. In these cases the introduction of an optically-thin hole in the inner part of the optical thick disc improves the agreement of the model with the observed SED (see Hil92), while the other parameters of the UV3C model retain their values. In Appendix A we give several observations and theoretical arguments that seem to support the UV3C-model.

Acknowledgements. The authors are grateful to F. Palla, P. Bernasconi, and L. Siess for providing us with the files of their models for the PMS evolution of intermediate mass stars. Along with the support by the VILSPA and GSFC Operational staff during the IUE observations, we are also indebted to the staff at the IUE spectral archives ULDA, INES, and NEWSIPS. We also thank the contributors to the photometric archive of Wesleyan Observatory, that of the ROTOR group at Maidanak Observatory (Uzb.), the LTPV-group of ESO observers (Sterken 1983), and the observations of de Winter et al. Photometry on the Walraven system was obtained at ESO. Most of the stellar distances were supplied by the Hipparcos Catalogue. The authors wish to thank the referee for valuable suggestions for improving this paper.

\section{References}

Adams, F. C., \& Shu, F. H. 1986, ApJ, 308, 836

Adams, F. C., Lada, C. J., \& Shu, F. H. 1987, ApJ, 312, 788

Adams, W. S., Joy, A. H., Humason, M. L., \& Brayton, A. M. 1935, ApJ, 81, 187

Akeson, R. L., Ciardi, D. R., van Belle, G. T., \& Creech-Eakman, M. J. 2002, ApJ, 566, 1124

Alencar, S. H. P., Melo, C. H. F., Dullemond, C. P., et al. 2003, A\&A, 409, 1037 van den Ancker, M. E. 1996, Master's Thesis University of Amsterdam The Evolutionary Status of Herbig Ae/Be Stars

van den Ancker, M. E., Thé, P. S., \& de Winter, D. 1996, A\&A, 309, 809

van den Ancker, M. E., Thé, P. S., Tjin A Djie, H. R. E., et al. 1997, A\&A, 324, L33 (ATT97)

van den Ancker, M. E., de Winter, D., \& Tjin A Djie, H. R. E. 1998, A\&A, 330, 145 (AWT98)

van den Ancker, M. E., Pérez, M. R., de Winter, D., \& McCollum, B. 2000, A\&A, $363, \mathrm{~L} 25$

van den Ancker, M. E., Blondel, P. F. C., Tjin A Djie, H. R. E., et al. 2004, MNRAS, 349, 1516

Andersen, J., Lindgren, H., Hazen, M. L., \& Mayor, M. 1989, A\&A, 299, 933 (And89)

Armitage, P. J., \& Clarke, C. J. 1996, MNRAS, 280, 458

Artyukhina, N. M. 1959, AZh, 36, 832 (SvA, 3, 808)

Artyukhina, N. M. 1960, AZh, 37, 95 (SvA, 4, 91)

Augereau, J. C., Langrange, A. M., Mouillet, D., \& Ménard, F. 2001, A\&A, 365, 78

Aveni, A. F., \& Hunter, J. H. 1969, AJ, 74, 1021

Baade, D., \& Stahl, O. 1989a, A\&A, 209, 255

Baade, D., \& Stahl, O. 1989b, A\&A, 209, 268

Barrado y Navascués, D., Stauffer, J. R., Song, I., \& Caillault, J.-P. 1999, ApJ, 520, L123

Barth, W., Weigelt, G., \& Zinnecker, H. 1994, A\&A, 291, 500

Barylak, M. 1989, IUE ESA Newsletter, 33, 20

Basri, G., \& Bertout, C. 1989, ApJ, 341, 340

Berdyugin, A. V., Berdyugina, S. V., Grinin, V. P., \& Minikulov, N. Kh. 1990, AZh, 67, 812 (SvA, 34, 408)

Berdyugin, A. V., Grinin, V. P., \& Minikulov, N. Kh. 1992, Izvestiya Krimskoi Astr. Obs., 86, 69

Bernacca, P. L., Lattanzi, M. G., Bucciarelli, B., et al. 1993, A\&A, 278, L47

Bernacca, P. L., Lattanzi, M. G., Porro, I., Neuhäuser, R., \& Bucciarelli, B. 1995, A\&A, 299, 933

Bernasconi, P. A. 1996, A\&AS, 120, 57

Bertout, C., Basri, G., \& Bouvier, J. 1988, ApJ, 330, 350 (BBB88)

Bertout, C., \& Regev, O. 1992, ApJ, 399, L163

Bertout, C., Robichon, N., \& Arenou, F. 1999, A\&A, 352, 574

Beskrovnaya, N. G., \& Pogodin, M. A. 1996, Astro. Lett., \& Comm., 34, 321, Adv. Study Inst. on Solar and Astrop. MHD flows, Heraklion, Greece 1995 Beskrovnaya, N. G., Pogodin, M. A., Miroshnichenko, A. S., et al. 1999, A\&A, 343,163

Bessel, M. S., \& Eggen, O. J. 1972, ApJ, 177, 209

Bibo, E. A., \& Thé, P. S. 1990, A\&A, 236, 155 
Blondel, P. F. C., \& Tjin A Djie, H. R. E. 1994, ASP Conf. Ser, 62, $211^{7}$

Blondel, P. F. C., \& Tjin ADjie, H. R. E. 1996, Lecture Notes in Physics, 465 (Heidelberg: Springer-Verlag), Proceedings Disks and Outflows around Young Stars, Heidelberg 5-9 september 1994, ed. S. V. W. Beckwith, J. Staude, A. Quetz, \& A. Natta

Blondel, P. F. C., Tjin A Djie, H. R. E., \& Thé, P. S. 1989, A\&A, 80, 115

Blondel, P. F. C., Talavera, A., \& Tjin A Djie, H. R. E. 1993, A\&A, 268, 624

de Boer, K. S. 1977, A\&A, 61, 605

Böhm, T., \& Catala, C. 1995, A\&A, 301, 155

Bond, H. E. 1970, PASP, 82, 1065

Bondi, H., \& Hoyle, F. 1944, MNRAS, 104, 273

Boyd, C. D. 1936, Harvard Bull., 904, 18

Brand, P. W. J. L., Hawarden, T. G., Longmore, A. J., Williams, P. M., \& Caldwell, J. A. R. 1983, MNRAS, 203, 215

Brandner, W., Bouvier, J., Grebel, E. K., et al. 1995, A\&A, 298, 818

Brandner, W., Lehman, T., Schöller, M., Weigelt, G., \& Zinnecker, H. 1996, ESO Mess., 83, 43

Brown, A., Tjin A Djie, H. R. E., Blondel, P. F. C., et al. 1997, ASP Conf. Ser., 121 (IAU Coll., 163), Accretion Phenomena and Related Outflow, Port Douglas, Queensland, 1996, ed. D. T. Wickramasinghe, L. Ferrario, \& G. V. Bicknell

Brunini, A., \& Benvenuto, O. G. 1996, MNRAS, 283, L84

Burbidge, E. M., \& Burbidge, O. J. 1954, ApJ, 119, 501

Cameron, A. C., \& Campbell, C. G. 1993, A\&A, 274, 309

Cameron, A. C., Campbell, C. G., \& Quaintrell, H. 1995, A\&A, 298, 133

Cannon, A. J. 1931, Harvard Ann., 100, 87

Cantó, J., D’Alessio, P., \& Lizano, S. 1995, RevMexAASC, 1, 217

Cardelli, J. A., Clayton, G. C., \& Mathis, J. S. 1988, ApJ, 329, L33

Cardelli, J. A., Clayton, G. C., \& Mathis, J. S. 1989, ApJ, 345, 245

Catala, C., Czarny, J., Felenbok, P., \& Praderie, F. 1986, A\&A, 154, 103

Catala, C., Simon, T., Praderie, F., et al. 1989, A\&A, 221, 273

Chauvin, G., Fusco, T., Lagrange, A.-M., et al. 2002, A\&A, 394, 223

Cincotta, P. M., Méndez, N., \& Núñes, J. A. 1995, ApJ, 449, 231

Claria, J. J. 1974, A\&A, 37, 229

Cohen, M., \& Schwartz, R. D. 1976, MNRAS, 174, 137

Cohen, M., \& Kuhi, L. V. 1979, ApJS, 41, 743 (CK79)

Corcoran, M., \& Ray, T. P. 1995, A\&A, 301, 729

Corporan, P., \& Lagrange, A.-M. 1999, A\&AS, 136, 429

Covino, E., Terranegra, L. V., Vittone, A. A., \& Russo, G. 1984, AJ, 89, 12

Crifo, F., Vidal-Madjar, A., Lallement, R., Ferlet, R., \& Gerbaldi, M. 1997, A\&A, 320, L29

Devine, D., Grady, C. A., Kimble, R. A., et al. 2000, ApJ, 542, L115

Donati, J.-F., Semel, M., Carter, B. D., Rees, D. E., \& Cameron, A. C. 1997 , MNRAS, 291, 658

Dragomiretskaya, B. A. 1965, Afz., 1, 455 (Astroph., 1, 241)

Dunkin, S. K., Barlow, M. J., \& Ryan, S. G. 1997a, MNRAS, 286, 604

Dunkin, S. K., Barlow, M. J., \& Ryan, S. G. 1997b, MNRAS, 290, 165

Dutrey, A. 2000, Workshop Herbig Ae/Be stars Amsterdam 25-27 Oct. 2000

Eaton, N. L., \& Herbst, W. 1995, AJ, 110, 2369

Eggen, O. J. 1998, AJ, 116, 1322

Eiroa, C., Oudmaijer, R. D., Davies, J. K., et al. 2002, A\&A, 384, 1038

Elias, J. H. 1978a, ApJ, 224, 453

Elias, J. H. 1978b, ApJ, 223, 859

Eugenio, M., \& Mendoza, V. 1966, ApJ, 143, L1010

Evans, A., Davies, J. K., Kilkenny, D., \& Bode, M. F. 1989, MNRAS, 237, 695

ESA 1997, The Hipparcos and Tycho Catalogues, ESA SP-1200

ESO Scientific Reports 8 (1991), 12 (1993), 14 (1994) \& 16 (1995)

Felenbok, P., Praderie, F., \& Talavera, A. 1983, A\&A, 128, 74

Fernández, M., \& Eiroa, C. 1995, A\&A, 310, 143

Fernández, M., Ortiz, E., Eiroa, C., \& Miranda, L. F. 1995, A\&AS, 114, 439

Finkenzeller, U. 1985, A\&A, 151, 340 (Fz85)

Finkenzeller, U., \& Jankovics, I. 1984, A\&AS, 57, 285 (FJ84)

Finkenzeller, U., \& Mundt, R. 1984, A\&AS, 55, 109

Friedemann, C., Reimann, H. G., Gürtler, J., \& Tóth, V. 1993, A\&A, 277, 184

Garrison, L. M. jr. 1978, ApJ, 224, 535

Glagolevskii, Y. V., \& Choutonov, G. A. 1998, Bull. Spec. Astrop. Obs., 45, 105 Godon, P. P. 1996, ApJ, 463, 674

Gómez de Castro, A. I., \& Fernández, M. 1996, MNRAS, 283, 55

Götz, W., \& Wenzel, W. 1968, Mit. Ver. St., 5, 13

Grady, C. A., Pérez, M. R., Thé, P. S., et al. 1995, A\&A, 302, 472

Grady, C. A., Pérez, M. R., Talavera, A., et al. 1996, A\&AS, 120, 157

Grady, C. A., Devine D., Woodgate B., et al. 2000, ApJ, 544, 895

Grinin, V. P. 1988, Pis'ma AZh, 14, 65 (SvA Lett., 14, 27)

7 Proceedings 1st Conference on the Nature and Evolutionary Status of Herbig Ae/Be Stars, Amsterdam 26-29 October 1993, ed. P. S. Thé, M. R. Pérez, \& E. P. J. van den Heuvel.
Grinin, V. P., \& Rostopchina, A. N. 1996, AZh, 73, 194 (Astr. Rep., 40, 171)

Grinin, V. P., Kiselev, N. N., Minikulov, N. Kh., \& Chernova, G. P. 1988, Pis'ma AZh, 14, 514 (SvA Lett., 34, 84)

Grinin, V. P., Kozlova, O. V., Natta, A., et al. 2001, A\&A, 379, 482

de Groot, M. 1971, ESO Ann. Rep., 32

Gurzadyan, G. A., \& Rustambekova, S. S. 1980, Ap\&SS, 69, 295

Haas, M., Christov, J. C., Zinnecker, H., Ridgway, S. T., \& Leinert, Ch. 1993, A\&A, 269, 282

Hamann, F., \& Persson, S. E. 1992, ApJS, 82, 285

Hartigan, P., \& Graham, J. H. 1987, ApJ, 93, 913

Hartmann, L., Kenyon, S. J., Hewett, R., et al. 1989, ApJ, 338, 1001

Hartmann, L. W., Kenyon, S. J., \& Calvet, N. 1993, ApJ, 407, 219

Heber, U., Hunger, K., Jonas, G., \& Kudritski, R. P. 1984, A\&A, 130, 119

Herbertz, R., Ungerechts, H., \& Winnewasser, G. 1991, A\&A, 249, 483

Herbig, G. H. 1954, Trans. IAU, 8, 805 (Hb54)

Herbig, G. H. 1960a, ApJS, 43, 337 (Hb60a)

Herbig, G. H. 1960b, ApJ, 131, 632 (Hb60b)

Herbig, G. H. 1966, Vistas Astron., 8, 109 (Hb66)

Herbig, G. H., \& Bell, K. R. 1988, Lick Obs. Bull., 1111 (HB88)

Herbst, W. 1994, ASP Conf. Ser., 62, $35^{7}$

Herbst, W., \& Shevchenko, V. S. 1999, AJ, 118, 1043

Herbst, W., Booth, J. F., Koret, D. L., et al. 1987, AJ, 94, 137

Herbst, W., Herbst, D. K., Grossman, E. J., \& Weinstein, D. 1994, AJ, 108, 1906

Hessman, F. V., Eislöffel, J., Mundt, R., et al. 1991, ApJ, 370, 384

Hillenbrand, L. A., Strom, S. E., Vrba, F. J., \& Keene, J. 1992, ApJ, 397, 613 (Hil92)

Hobbs, L. M. 1974, ApJ, 191, 381

Hu, J. Y. Thé, P. S., \& de Winter, D. 1989, A\&A, 208, 213

Hu, J. Y., Blondel, P. F. C., Catala, C., et al. 1991, A\&A, 248, 150

Hughes, J., Hartigan, P., \& Clampitt, L. 1993, AJ, 105, 571

Hutchinson, M. G., Albinson, J. S., Barrett, P., et al. 1994, A\&A, 285, 883

Imhoff, C., \& Wasatonic, R. 1986, IUE NASA Newsletter, 29, 45

Irvine, N. J. 1975, PASP, 87, 78

Jaschek, M., Jaschek, C., \& Andrillat, Y. 1988, A\&AS, 72, 505

Jayawardhana, R., \& Brandeker, A. 2001, ApJ, 561, L111

Johnson, H. M. 1964, ApJ, 142, 964

Joncour, I., Bertout, C., \& Bouvier, J. 1994, A\&A, 291, L19

Joy, A. H. 1949, ApJ, 110, 424

Kenyon, S. J., Hartmann, L. W., \& Hewitt, R. 1988, ApJ, 325, 231

Kenyon, S. J., Hartmann, L. W., Imhoff, C., \& Cassatella, A. 1989, ApJ, 344, 925

Kholopov, P. N. 1959, AZh, 36, 295 (SvA, 3, 291) (Kh59)

Kholopov, P. N. 1985, Gen. Cat. of Var. Stars 4th Ed. (Moscow: Nauka Pub.) (Kh85)

Kilkenny, D., Whittet, D. C. B., Davies, J. K., et al. 1985, South Afr. Obs. Circ., 9,55

Kley, W., \& Lin, D. N. C. 1996, ApJ, 461, 933

Knee, L. B. G., \& Prusti, T. 1996, A\&A, 312, 455

Koen, C. 2003, MNRAS, 341, 1385

Kolotilov, E. A., \& Zajtseva, G. V. 1974, Afz, 10, 365 (Astroph., 10, 228)

Kondo, Y., \& Bruhweiler, F. C. 1985, ApJ, 291, L1

Koresko, C. D., Beckwith, S. V. W., Ghez, A. M., Matthews, K., \& Neugebauer, G. 1991, AJ, 102, 2073

Koval'chuk, G. H. 1984, IBVS, 248, 2 (Konkoly Obs. Budapest)

Koval'chuk, G. H. 1985, Afz, 23, 255 (Astroph., 23, 514)

Koval'chuk, G. H. 1986, Afz, 24, 213 (Astroph., 24, 213)

Kozlova, O. V., Grinin, V. P., \& Rostopchina, A. N. 2000, AZh, 77, 1 (Astron. Rep., 44, 1)

Krautter, J. 1980, A\&A, 89, 74

Krautter, J. 1991, Low Mass Star Formation in Southern Molecular Clouds, ESO Rep., 11, 127

Kurtz, D. W., \& Marang, F. 1995, MNRAS, 276, 191

Kurtz, D. W., \& Müller, M. 1999, MNRAS, 310, 1071

Kurtz, D. W., \& Müller, M. 2001, MNRAS, 325, 1341

Kurucz, R. L. 1979, ApJS, 40, 1

Kurucz, R. L. 1991, in Precision Photometry: Astrophysics of the Galaxy, ed. A. G. Davis Philip, A. R. Upgren, \& K. A. Janes (Schenectady, New York: L. Davis press)

Lanz, T., Heap, S. R., \& Hubeny, I. 1995, ApJ, 447, L41

Leinert, Ch., Richichi, A., Weitzel, N., \& Haas, M. 1994, ASP Conf. Ser., 62, $155^{7}$

Leinert, Ch., Richichi, A., \& Haas, M. 1997, A\&A, 318, 472

Lioure, A., \& Le Contel, O. 1994, A\&A, 285, 185

Lub, J., \& Pel, J. W. 1977, A\&A, 54, 137

Makarov, V., Bastian, U., Hoeg, E., Grossmann, V., \& Wicenec, A. 1994, IAU IBVS, 4118,1

Mannings, V., \& Sargent, A. I. 1997, ApJ, 490, 792

Malfait, K., Bogaert, E., \& Waelkens, C. 1998, A\&A, 331, 211 (MBW98) 
Marconi, M., Ripepi, V., Alcalá, J. M., et al. 2000, A\&A, 355, L35

Martin, E. L. 1993, PASP, 105, 277

Merrill, P. W., Burwell, C. G., \& Miller, W. C. 1942, ApJ, 96, 15

Meeus, G., Waelkens, C., \& Malfait, K. 1998, A\&A, 329, 131 (MWM98)

Mihalas, D. 1966, ApJS, 13, 1

Miroshnichenko, A. S., Bergner, Yu. K., \& Kuratov, K. S. 1997, Pis'ma AZh, 23,118 (SvA Lett., 23, 97)

Mora, A., Merin, B., Solano, E., et al. 2001, A\&A, 378, 116

Morrison, N. D. 1997 (priv. comm.)

Murdin, P., \& Penston, M. V. 1977, MNRAS, 181, 657

Muzerolle, J., Luhman, K. L., Briceño, C., Hartman, L., \& Calvet, N. 2005, ApJ, 625,906

NASA RP-1190 1988, IRAS Catalogs and Atlases Explanatory Supplement, ed. C. Beichman, G. Neugebauer, H. J. Habing, P. E. Clegg, \& T. J. Chester (Washington, DC: GPO), Vol. 1

Neuhäuser, R., Guenther, E., Mugrauer, M., Ott, Th., \& Eckart, A. 2002, A\&A, 395,877

$\mathrm{Ng}$, Y. K. 1986 (priv. comm.)

Olsen, E. H. 1979, A\&AS, 37, 367

Oudmaijer, R. D., van der Veen, W. E. C. J., Waters, L. B. F. M., et al. 1992, A\&AS, 96, 625 (Oud92)

Padoan, P., Kritsuk, A., \& Norman, M. L. 2005, ApJ, 662, L61

Palla, F. 1997 (priv. comm.)

Palla, F., \& Stahler, S. W. 1993, ApJ, 418, 414 (PS93)

Palla, F., \& Stahler, S. W. 1999, ApJ, 525, 772

Paresce, F. 1991, A\&A, 247, L25

Penston, M. V., Allen, D. A., \& Lloyd, Ch. 1976, The Observatory, 96, 22

Petrov, P. P., Gullbring, E., Ilyin, I., et al. 1996, A\&A, 314, 821

Pérez, M. R. 1991, IUE NASA Newsletter, 38, 27

Pérez, M. R., Grady, C. A., \& Thé, P. S. 1993, A\&A, 274, 381

Pérez, M. R., van den Ancker, M. E., de Winter, D., \& Bopp, B. W. 2004, A\&A, 416, 647

Pinheiro, F. J. G., Folha, D. F. M., Marconi, M., et al. 2003, A\&A, 399, 271

Pirzkal, N., Spillar, E. J., \& Dyck, H. M. 1997, ApJ, 481, 392

Plavec, M. J., Weiland, J. L., \& Koch, R. H. 1982, ApJ, 256, 206

Plavec, M. J. 1983, ApJ, 275, 251

Pogodin, M. A. 1987, Pis'ma AZh, 13, 695 (SvA Lett., 13, 293)

Pogodin, M. A. 1995, Ap\&SS, 224, 539

Popham, R., Narayan, R., Hartmann, L. W., \& Kenyon, S. J. 1993, ApJ, 415, L127

Regev, A., \& Bertout, C. 1995, MNRAS, 272, 71

Remijn, L. 1980, Master's Thesis University of Amsterdam, Herbig Ae/Be stars

Reipurth, B. 1983, A\&A, 117, 183

Reipurth, B., Pedrosa, A., \& Lago, M. T. V. T. 1996, A\&AS, 120, 229

Rossi, C., Errico, L., Friedjung, M., et al. 1999, A\&AS, 136, 95

Rydgren, A. E. 1980, AJ, 85, 444

Rydgren, A. E., Strom, S. E., \& Strom, K. M. 1976, ApJS, 30, 307

Schmidt-Kaler, Th. 1982, in Landolt-Börnstein Tables, Vol. VI/2b, SpringerVerlag (SK82)

Schöller, M., Brandner, W., Lehman, T., Weigelt, G., \& Zinnecker, H. 1996, A\&A, 315, 445

Shevchenko 1989, Herbig Ae/Be stars, Tashkent Ed. FAN Academic Sciences Uzbekistan (in Russian)

Shevchenko, V. S. 1994, AZh, 71, 572 (Astron. Rep., 38, 505)

Shevchenko, V. S. 1998, AZh, 76, 289 (Astron. Rep., 43, 246)

Shevchenko, V. S., Grankin, K. N., Ibrahimov, M. A., Melnikov, S. Yu., \& Yakubov, S. O. 1993, Ap\&SS, 202, 121

Shevchenko, V. S., Ezhkova, O. V., Tjin A Djie, H. R. E., et al. 1997, A\&AS, 124,33

Shevchenko, V. S., Ezhkova, O. V., Ibrahimov, M. A., van den Ancker, M. E., \& Tjin A Djie, H. R. E. 1999, MNRAS, 310, 210

Shore, S. N., \& King, A. R. 1986, A\&A, 154, 263
Siess, L., Forestini, M., \& Bertout, C. 1999, A\&A, 342, 480 Siess, L., Dufour, M., \& Forestini, M. 2000, A\&A, 358, 593 Slettebak, A. 1982, ApJS, 50, 55

Smith, B. A., \& Terrile, R. J. 1984, Science, 226, 1421

Stecklum, B., Eckart, A., Henning, T., \& Loewe, M. 1995, A\&A, 296, 463

Steenman, H., \& Thé, P. S. 1989, Ap\&SS, 159, 189

Sterken, C. 1983, ESO Mess., 33, 10

Strand, K. A. 1958, ApJ, 128, 14

Strom, S. E., Strom, K. M., Yost J., Carrasco L., \& Grasdalen, G. 1972, ApJ, $173,353(\operatorname{Str} 72)$

Strom, K. M., Strom, S. E., Carrasco L., \& Vrba, F. J. 1975, ApJ, 196, 489

Strömgren, B. 1948, ApJ, 108, 242

Sylvester, R. J., Barlow, M. J., \& Skinner, C. J. 1994, Ap\&SS, 212, 261

Sylvester, R. J., Skinner, C. J., Barlow, M. J., \& Mannings, V. 1996, MNRAS, 279,915

Talavera, A., Blondel, P. F. C., \& Tjin A Djie, H. R. E. 1994, ASP Conf. Ser., 62, $115^{7}$

Tambotseva, L. V., Grinin, V. P., Rodgers B., \& Koslova, O. V. 2001, AZh, 78, 514 (Astron. Rep., 45, 442)

Tessier, E., Bouvier, J., Beuzit, J. L., \& Brandner, W. 1994, ESO Mess., 78, 1

Testi, L., Palla, F., \& Natta, A. 1998, A\&AS, 133, 81

Thé, P. S., \& Tjin A Djie, H. R. E. 1978, A\&A, 62, 439

Thé, P. S., Tjin A Djie, H. R. E., Bakker R., et al. 1981, A\&AS, 44, 451

Thé, P. S., Felenbok, P., Cuypers, H., \& Tjin A Djie, H. R. E. 1985a, A\&A, 149, 429

Thé, P. S., Tjin A Djie, H. R. E., Catala, C., Praderie, F., \& Felenbok, P. 1985b, ESO Mess., 41, 8

Thé, P. S., Tjin A Djie, H. R. E., Steenman, H., \& Wesselius, P. R. 1986, A\&A, 155,347

Thé, P. S., de Winter, D., \& Pérez, M. R. 1994, A\&AS, 104, 315 (TWP94)

Thé, P. S., Pérez, M. R., Voshchinnikov, N. V., \& van den Ancker, M. E. 1996, A\&A, 314, 233

Thiébaut, E., Bouvier, J., Blazit, A., Bonneau, D., Foy, F. C., \& Foy, R. 1995, A\&A, 303, 795

Tjin A Djie, H. R. E., Remijn, L., \& Thé, P. S. 1984, A\&A, 134, 273

Tjin A Djie, H. R. E., Thé, P. S., Andersen, J., et al. 1989, A\&AS, 78, 1

Tjin A Djie, H. R. E., Blondel, P. F. C., Brown, A., \& Harper, G. M. 1998, ESASP, 413, 133, Proceedings Ultraviolet Astrophysics, Beyond the IUE Final Archive, Sevilla, Spain, November 11-14, 1997

Tjin A Djie, H. R. E., \& Blondel, P. F. C., in preparation (Paper II)

Ungerechts, H., \& Thaddeus, P. 1987, ApJS, 63, 645

Valenti, J. A., Johns-Krull, C. M., \& Linsky, J. L. 2000, ApJS, 129, 399

Vidal-Madjar, A., Lagrange-Henri, A.-M., Feldman, P. D., et al. 1994, A\&A, 290,245

Voshchinnikov, N. V. 1989, Afz, 30, 509 (Astroph., 30, 313)

Voshchinnikov, N. V., \& Karjukin, V. V. 1994, A\&A, 288, 883

Walker, H. J., \& Wolstencroft, R. D. 1988, PASP, 100, 1509 (WW88)

Wallenquist, A. 1937, Uppsala Medd., 71

Walter, F. M., Brown, A., Linsky, J. L., et al. 1987, ApJ, 314, 297

Wamsteker, W., Driessen, C., Muñoz, J. R., et al. 1989, A\&AS, 79, 1

Warren, W. H., \& Hesser, J. E. 1978, ApJS, 36, 497

Weaver, W. M. B., \& Hobson, S. W. 1988, PASP, 100, 1443

Whitney, B. A., Clayton, G. C., Schulte-Ladbeck, R. E., et al. 1993, ApJ, 417, 687

de Winter, D. 1998 (priv. comm.)

de Winter, D., \& Thé, P. S. 1990, Ap\&SS, 166, 99

de Winter, D., \& van den Ancker, M. R. 1991 (priv. comm.)

de Winter, D., van den Ancker, M. E., Maira A., et al. 2001, A\&A, 380, 609

Yi, I. 1994, ApJ, 428, 760

Zajtseva, G. V. 1973, Per. Zv., 19, 63

Zajtseva, G. V. 1986, Afz., 25, 471 (Astroph., 25, 626)

Zajtseva, G. V., \& Kolotilov, E. A. 1973, Afz, 9, 185 (Astroph., 9, 104) 
P. F. C. Blondel and H. R. E. Tjin A Djie: Modeling of PMS Ae/Fe stars using UV spectra, Online Material $p 1$

\section{Online Material}


P. F. C. Blondel and H. R. E. Tjin A Djie: Modeling of PMS Ae/Fe stars using UV spectra, Online Material $p 2$
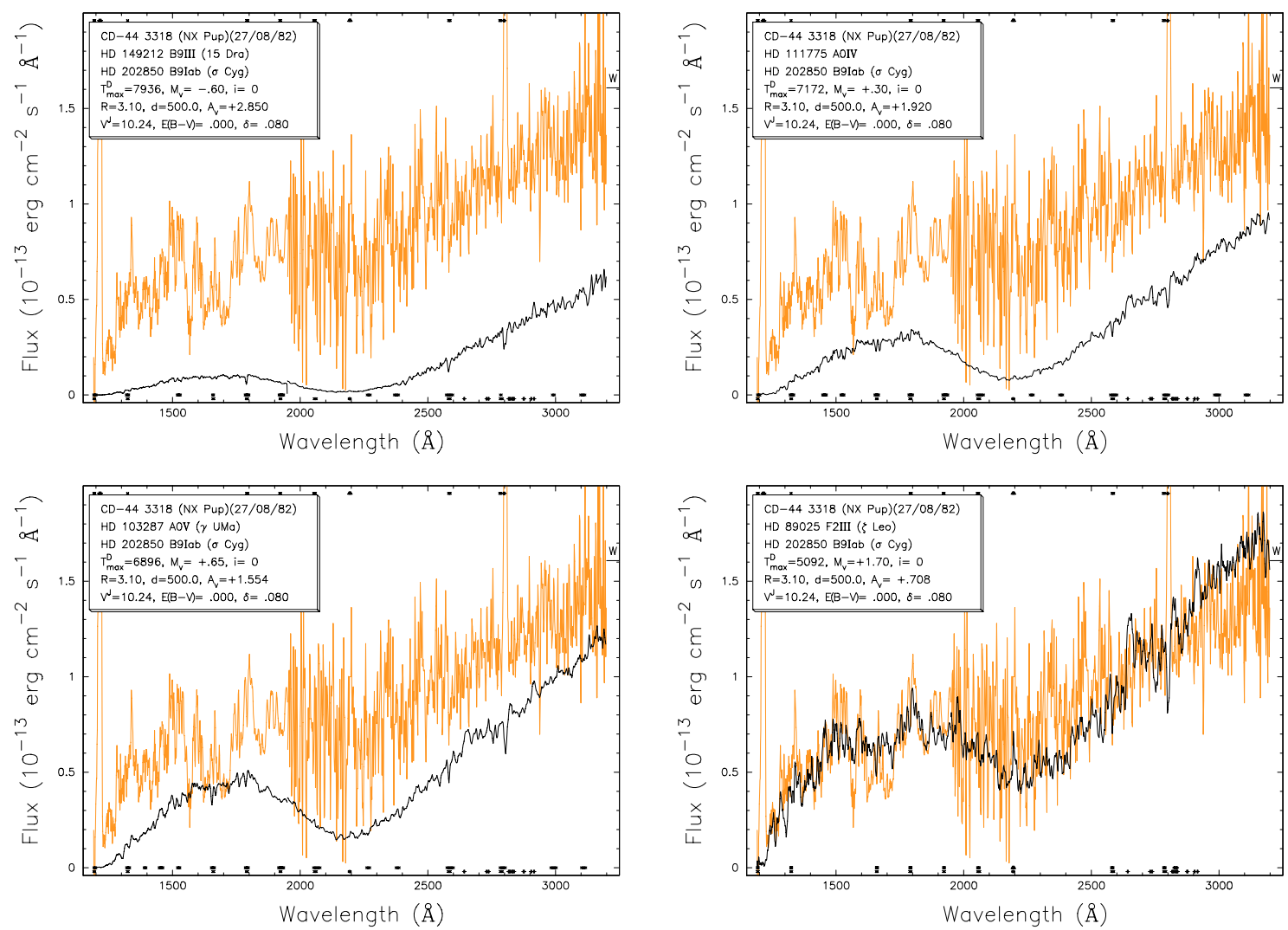

Fig. 4. Model calculations with 4 different spectral types, each with the standard $M_{\mathrm{v}}$-value (SK82).
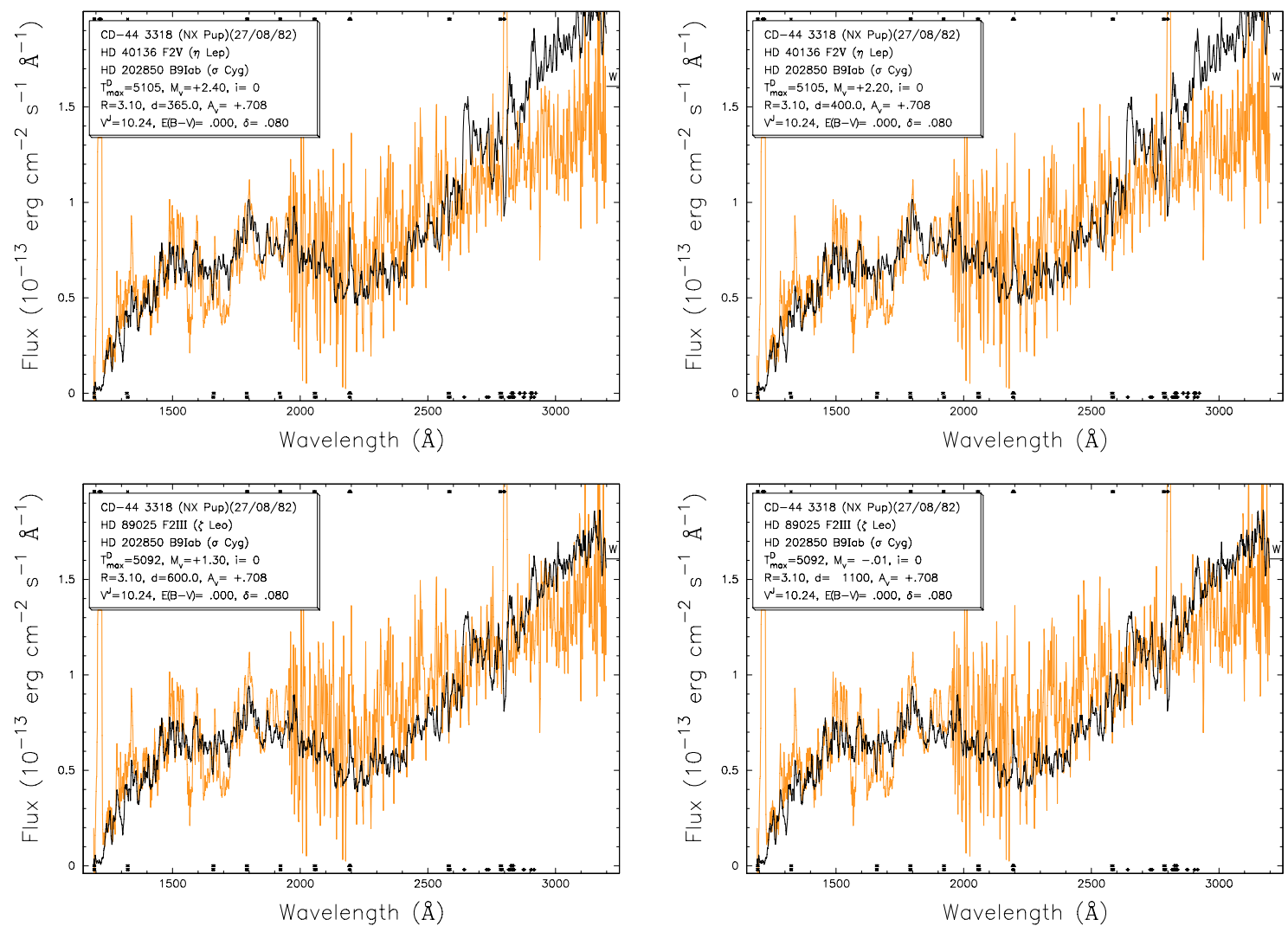

Fig. 5. Model "solutions" for NX Pup with 4 different $M_{\mathrm{v}}$ and $d$ combinations so that $A_{\mathrm{v}}^{\mathrm{cs}} \approx 0.708$. 
P. F. C. Blondel and H. R. E. Tjin A Djie: Modeling of PMS Ae/Fe stars using UV spectra, Online Material p 3
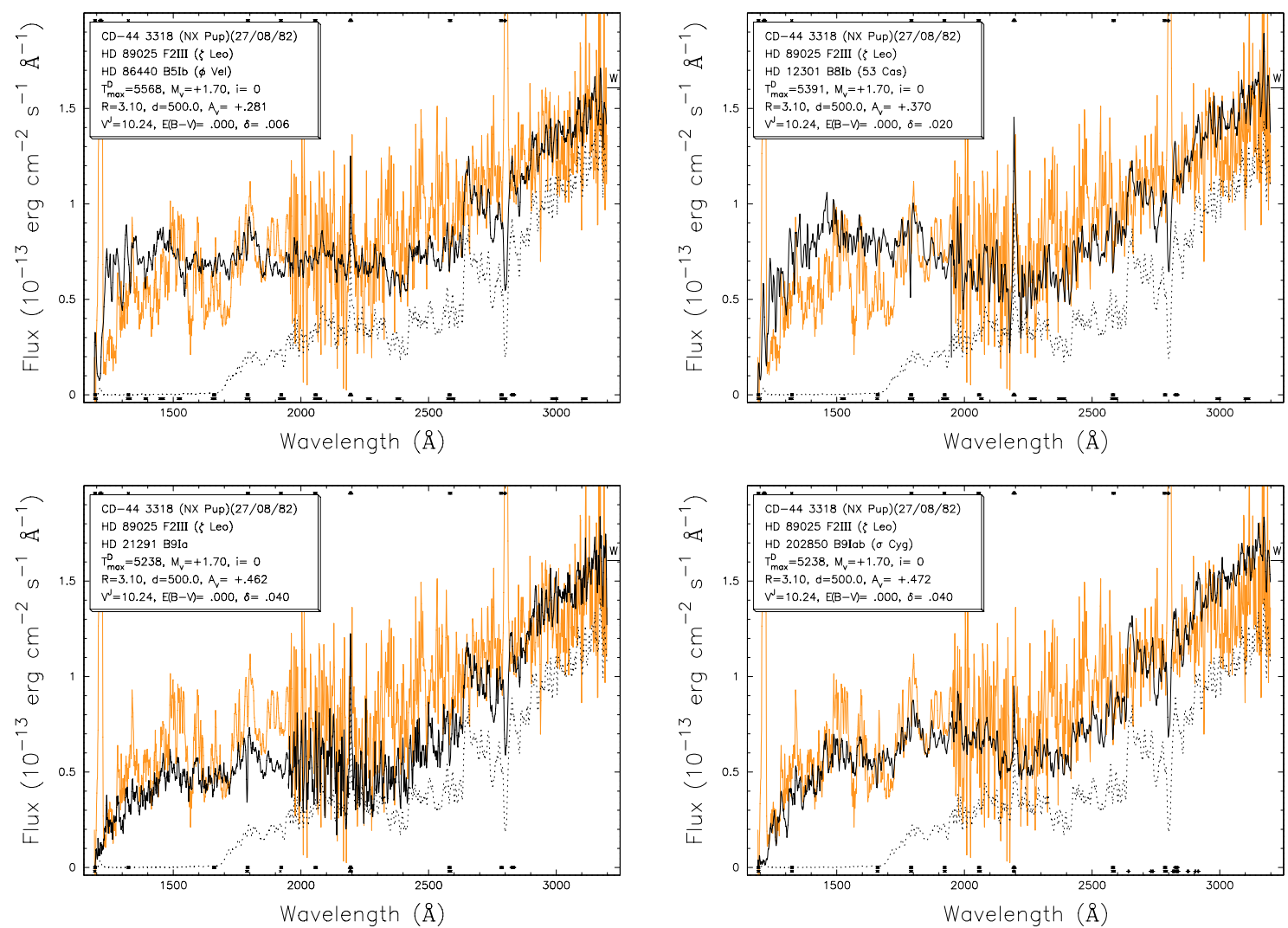

Fig. 6. Model calculations for NX Pup with 4 different BL types and sizes.
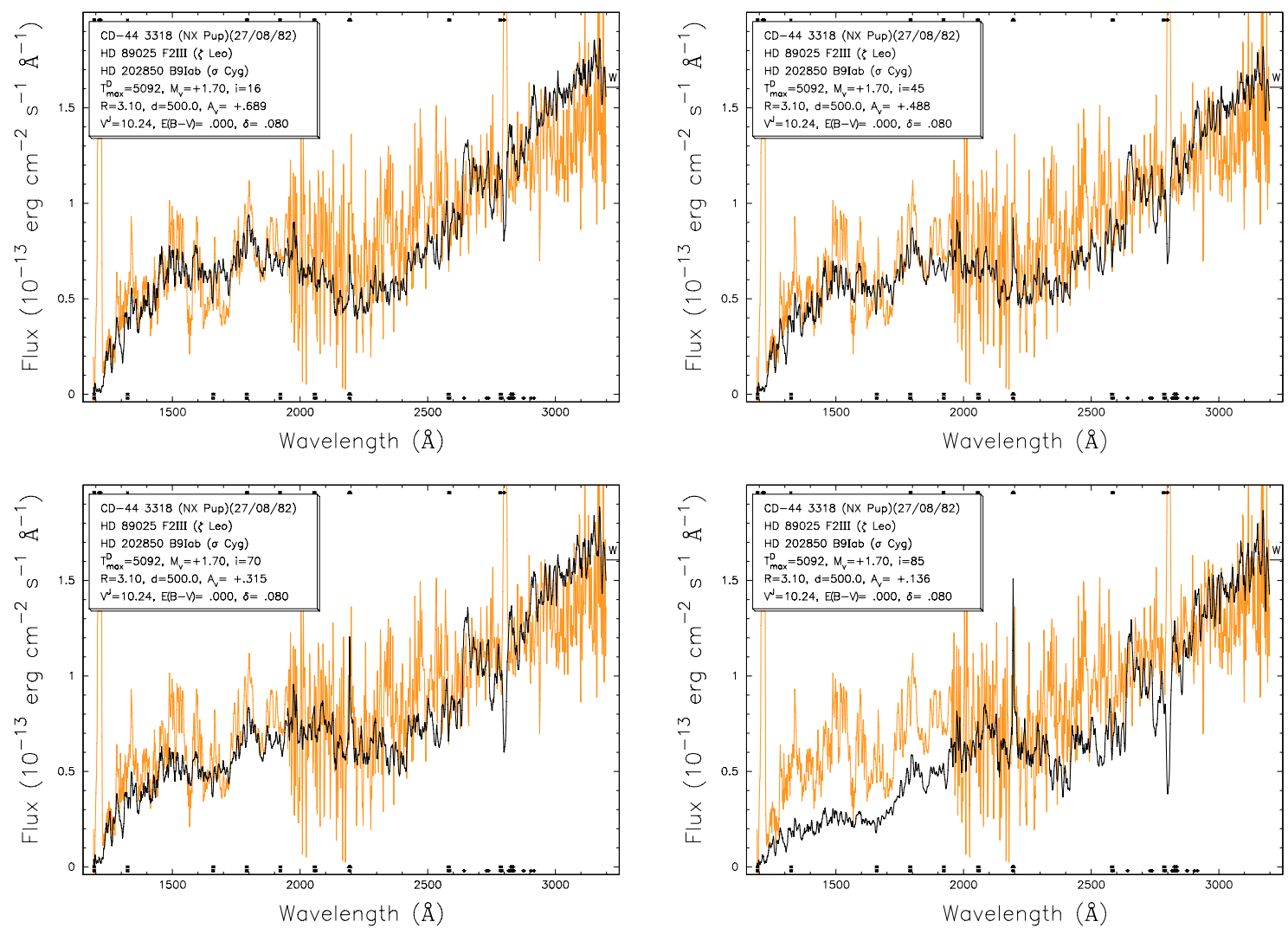

Fig. 7. Model calculations for NX Pup with $i=16^{\circ}, 45^{\circ}, 70^{\circ}$ and $85^{\circ}$. 
P. F. C. Blondel and H. R. E. Tjin A Djie: Modeling of PMS Ae/Fe stars using UV spectra, Online Material p 4
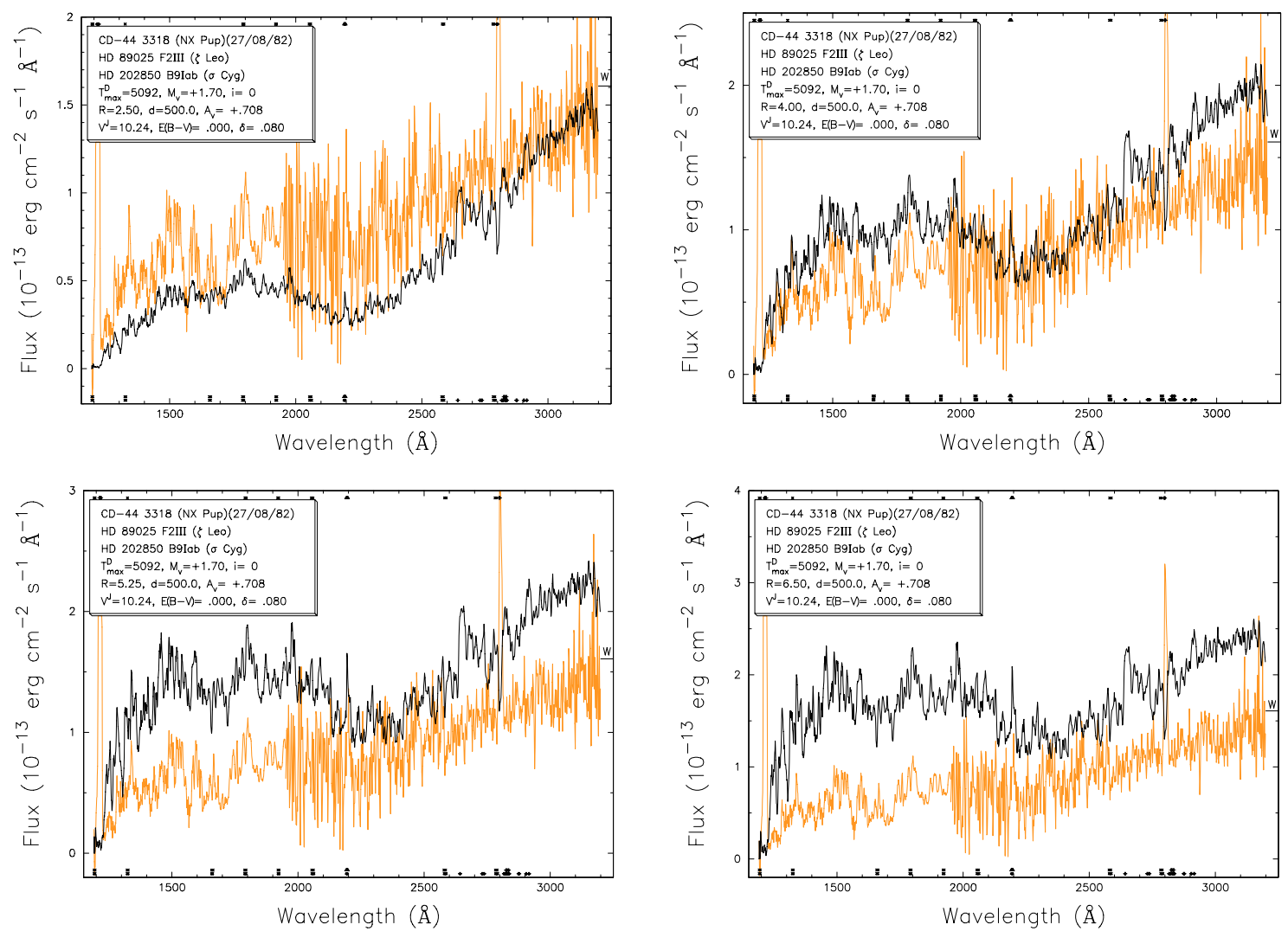

Fig. 8. Model calculations for NX Pup with $4 R^{\mathrm{cs}}$-values.
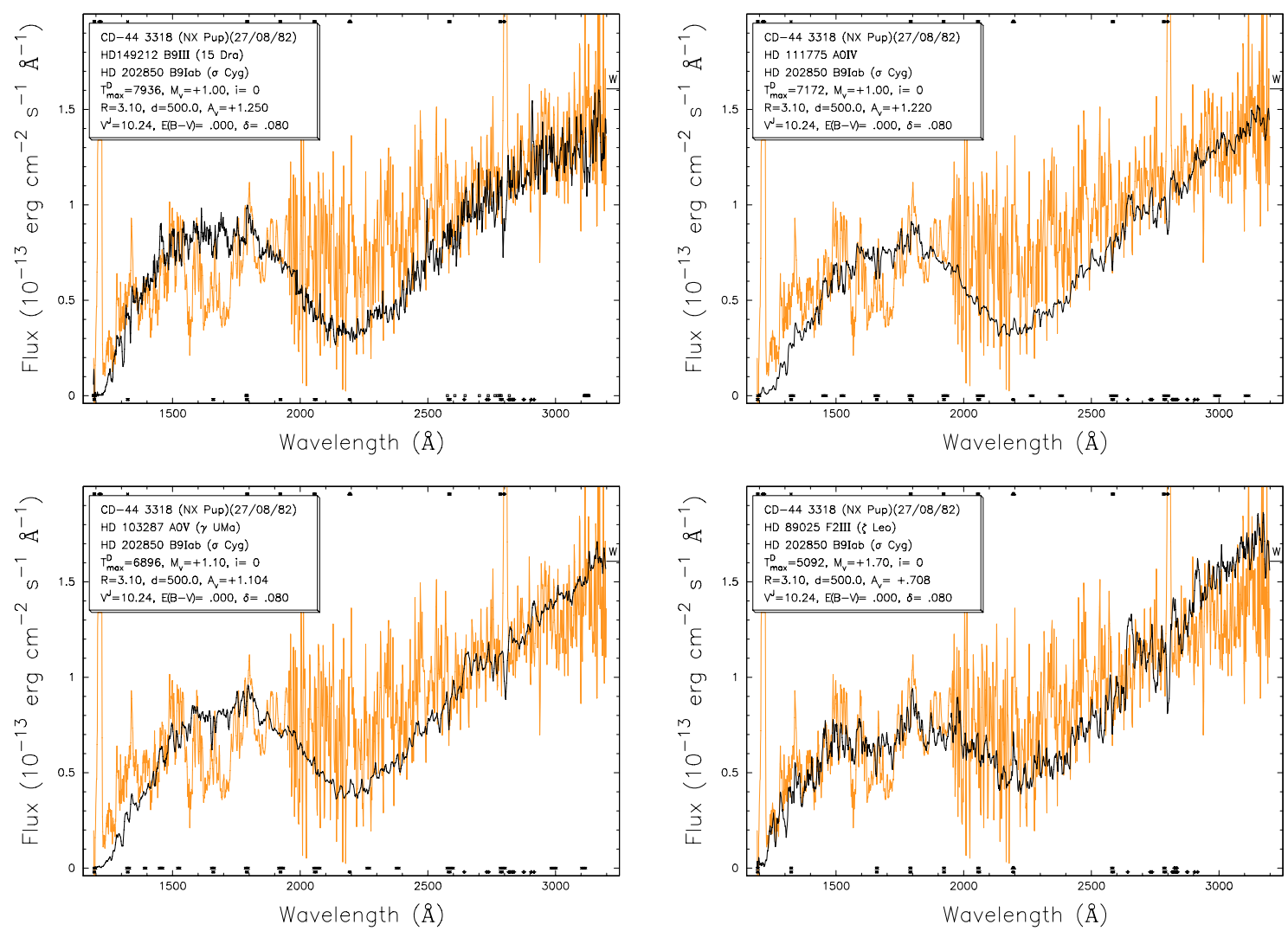

Fig. 9. As Fig. 4 but with changed $M_{\mathrm{v}}$-values to match the observed spectrum. 
P. F. C. Blondel and H. R. E. Tjin A Djie: Modeling of PMS Ae/Fe stars using UV spectra, Online Material p 5

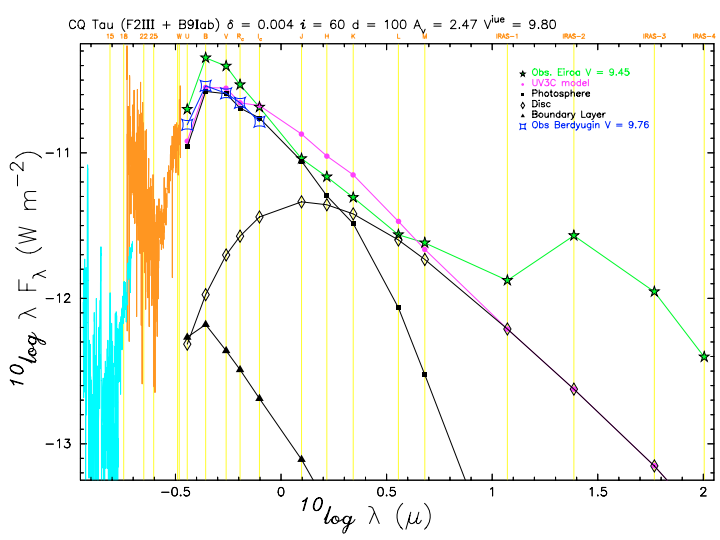

Fig. 33. CQ Tau F2 III.

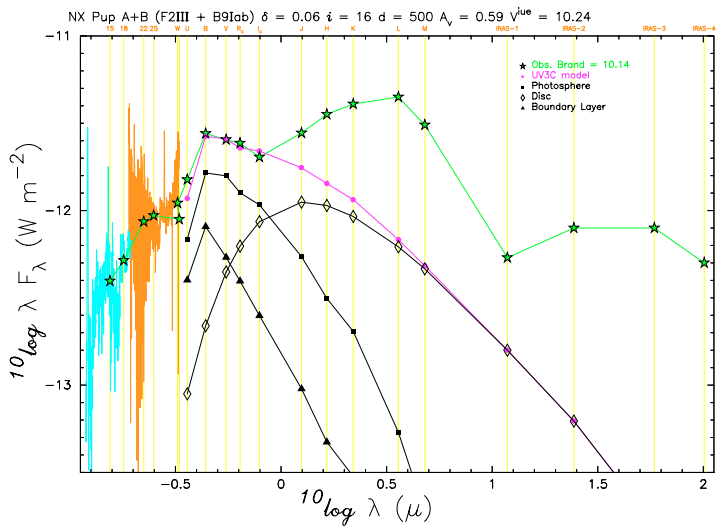

Fig. 34. NX Pup F2 III.

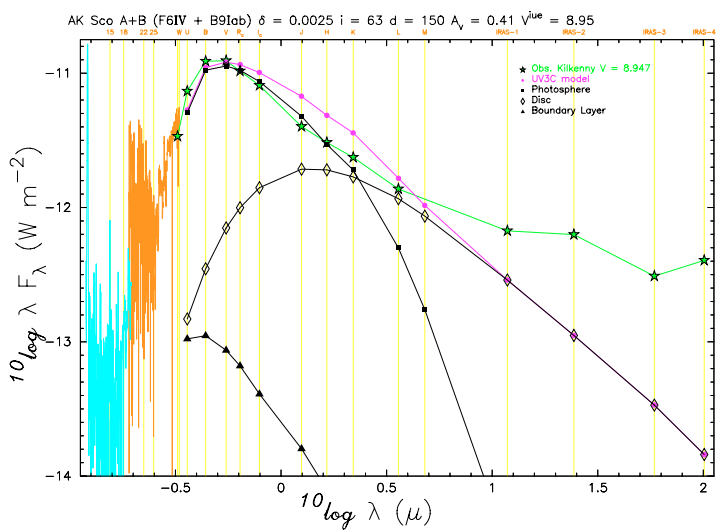

Fig. 35. AK Sco F6 IV.

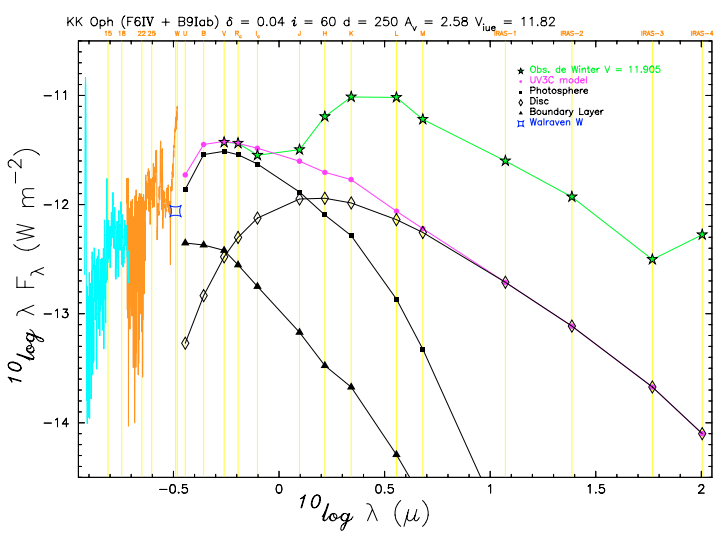

Fig. 36. KK Oph F6IV.

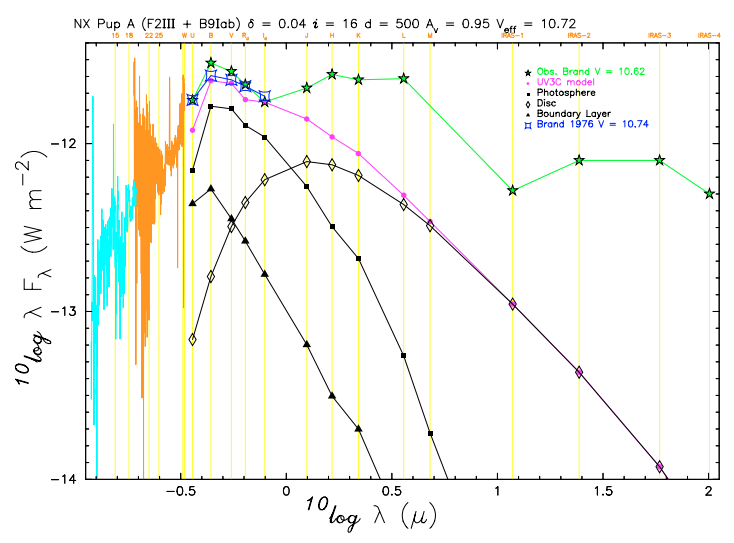

Fig. 37. NX Pup A F2 III.

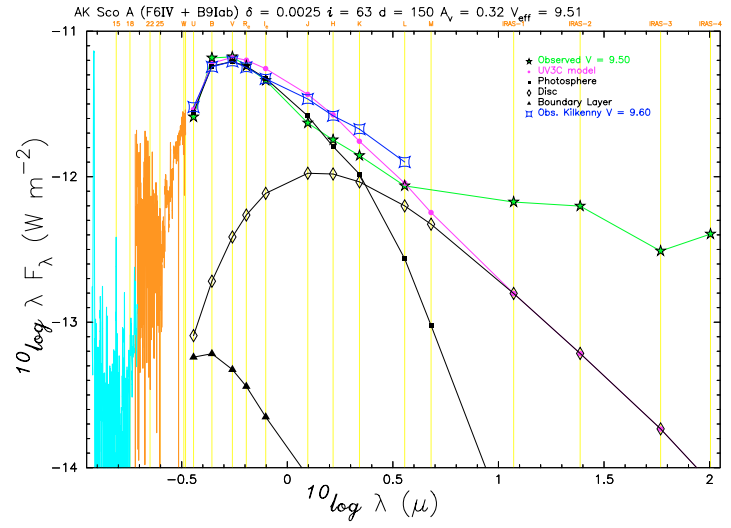

Fig. 38. AK Sco A F6 IV.

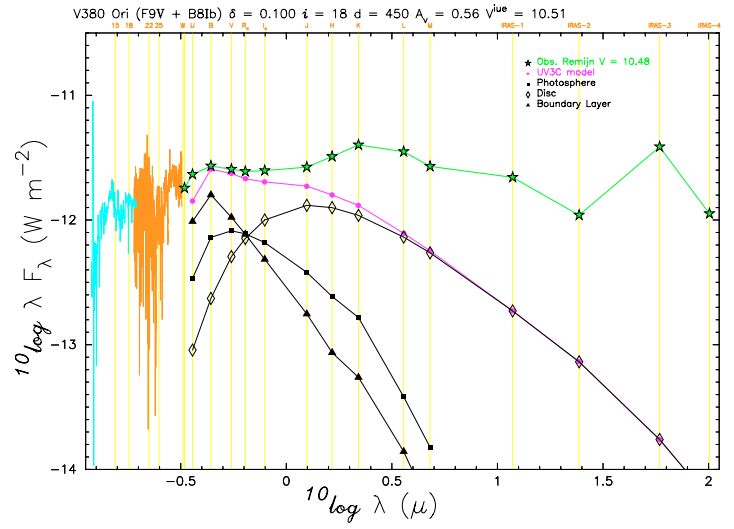

Fig. 39. V380 Ori F9 v.

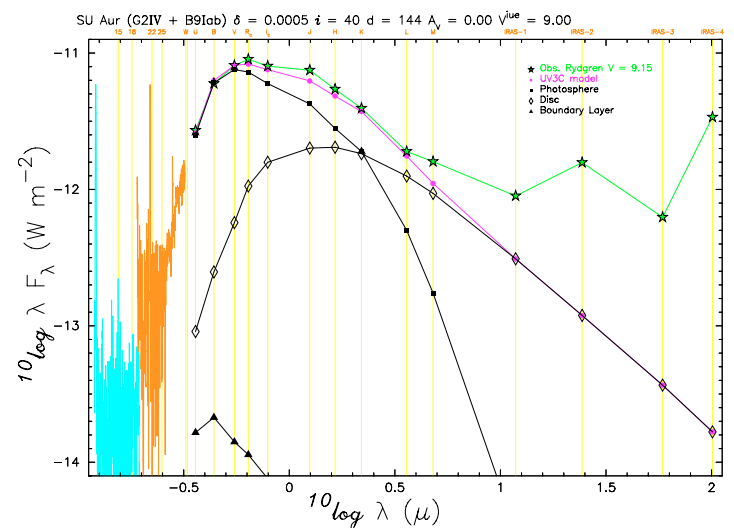

Fig. 40. SU Aur G2 IV. 
P. F. C. Blondel and H. R. E. Tjin A Djie: Modeling of PMS Ae/Fe stars using UV spectra, Online Material p 6

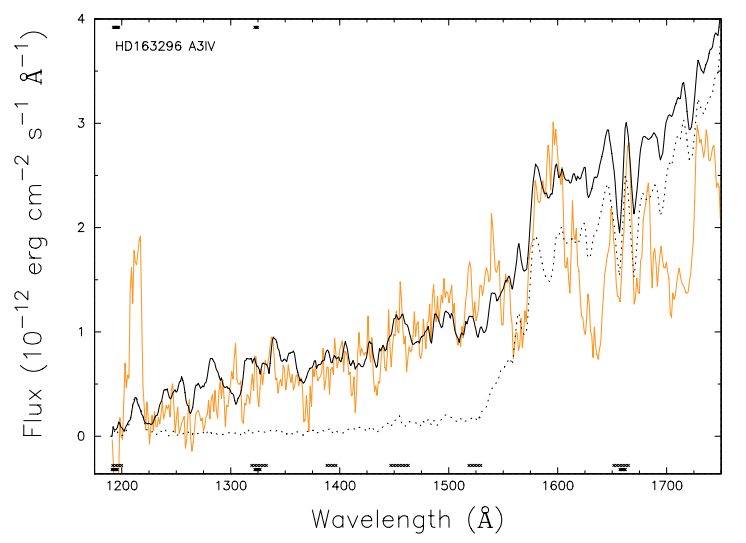

Fig. 45. HD 163296 A3 IV.

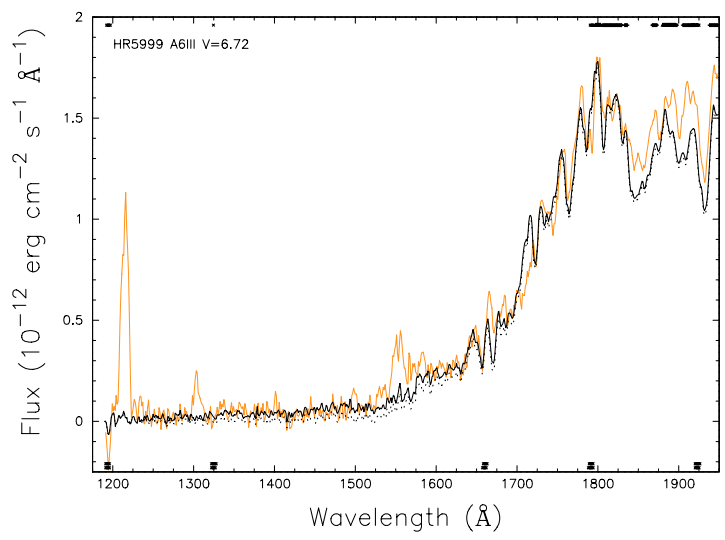

Fig. 46. HR 5999 A6 III.

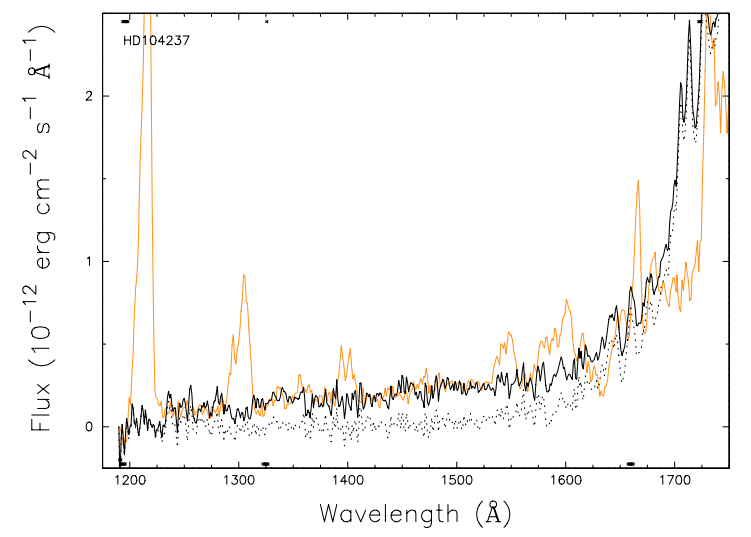

Fig. 47. HD 104237 A7 IV.

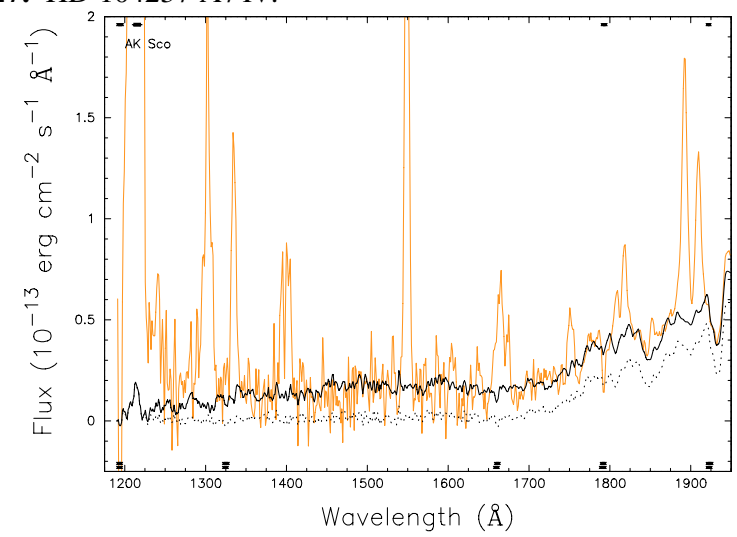

Fig. 48. AK Sco F6 IV.

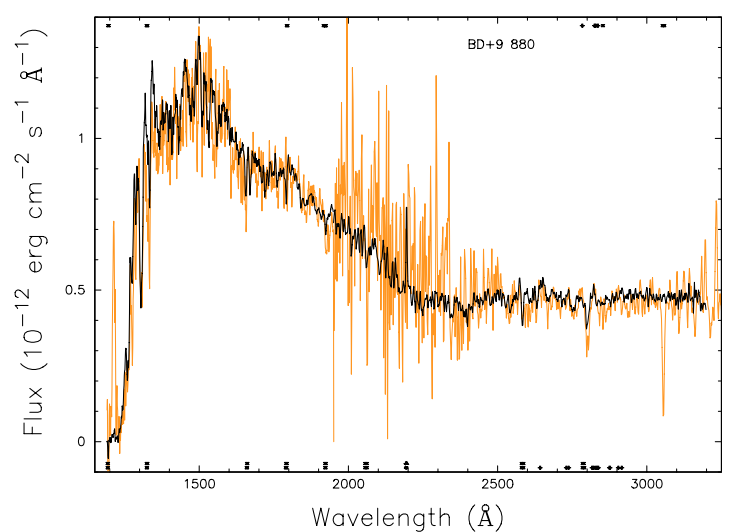

Fig. 49. $\mathrm{BD}+09^{\circ} 880 \mathrm{~B} 9 \mathrm{Vz}$.

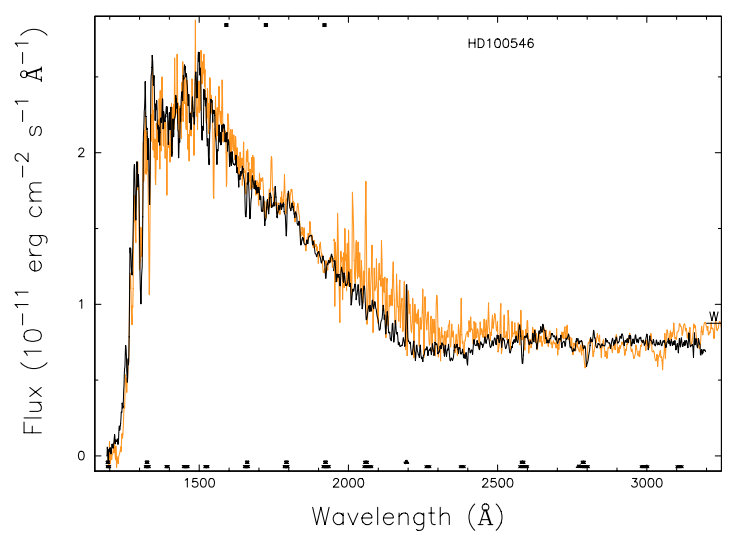

Fig. 50. HD 100546 B9 v.

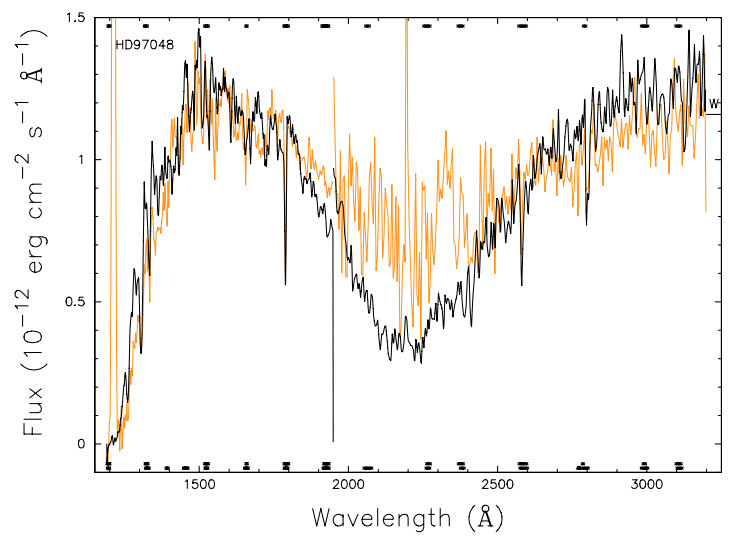

Fig. 51. HD 97048 B9 III.

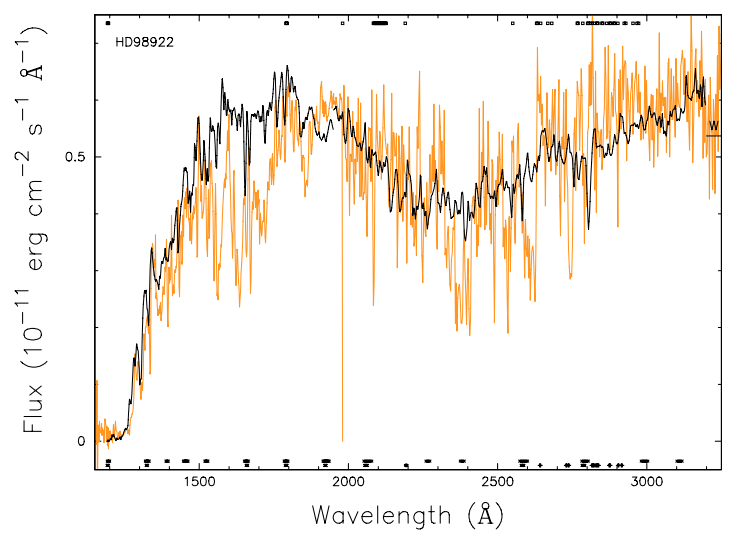

Fig. 52. HD 98922 A0 Vz. 
P. F. C. Blondel and H. R. E. Tjin A Djie: Modeling of PMS Ae/Fe stars using UV spectra, Online Material $p 7$

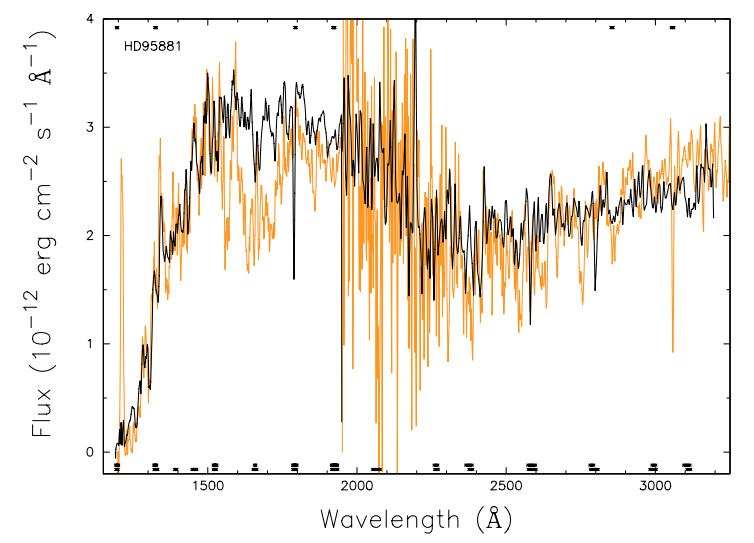

Fig. 53. HD 95881 A1 III.

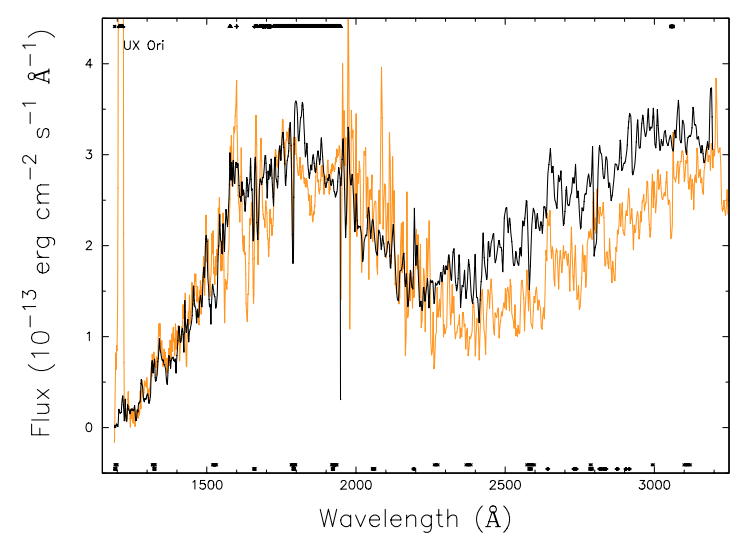

Fig. 54. UX Ori A2 III.

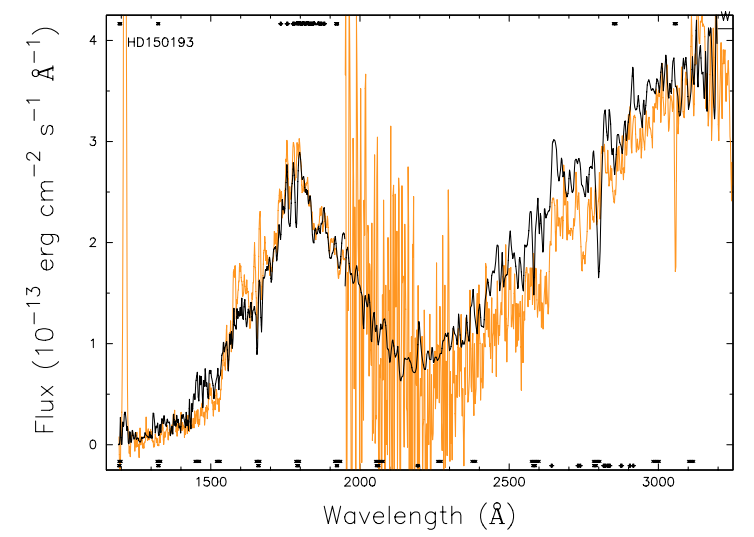

Fig. 55. HD 150193 A3 III.

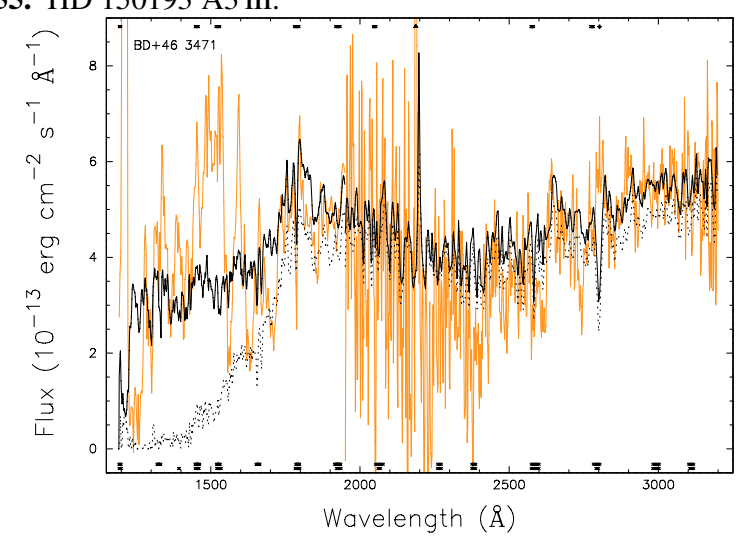

Fig. 56. $\mathrm{BD}+46^{\circ} 3471 \mathrm{~A} 3 \mathrm{III}$.

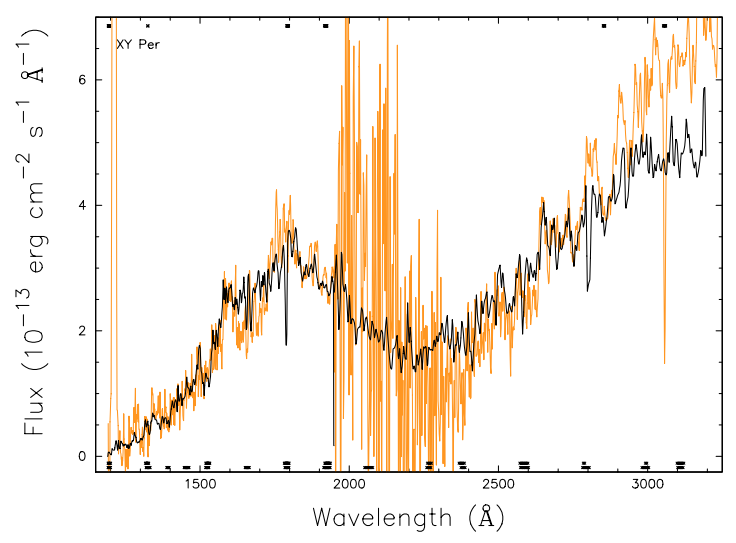

Fig. 57. XY Per A2 III.

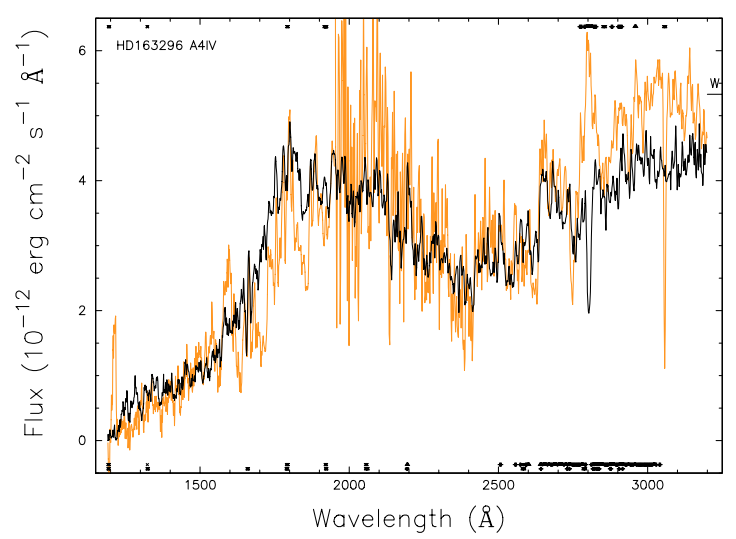

Fig. 58. HD 163296 A4 IV.

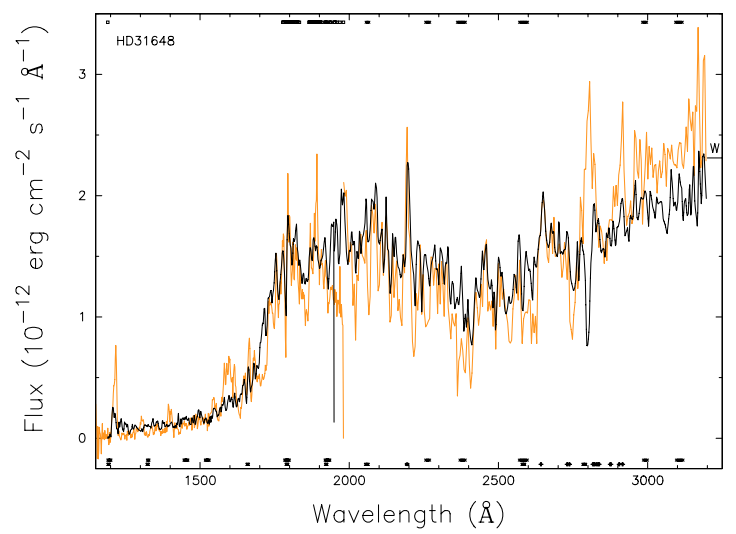

Fig. 59. HD 31648 A5 Vz.

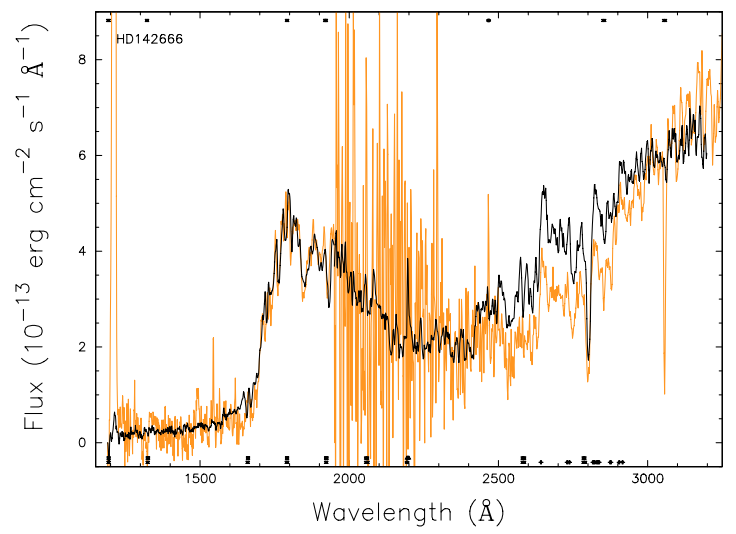

Fig. 60. HD 142666 A7 III. 
P. F. C. Blondel and H. R. E. Tjin A Djie: Modeling of PMS Ae/Fe stars using UV spectra, Online Material $p 8$

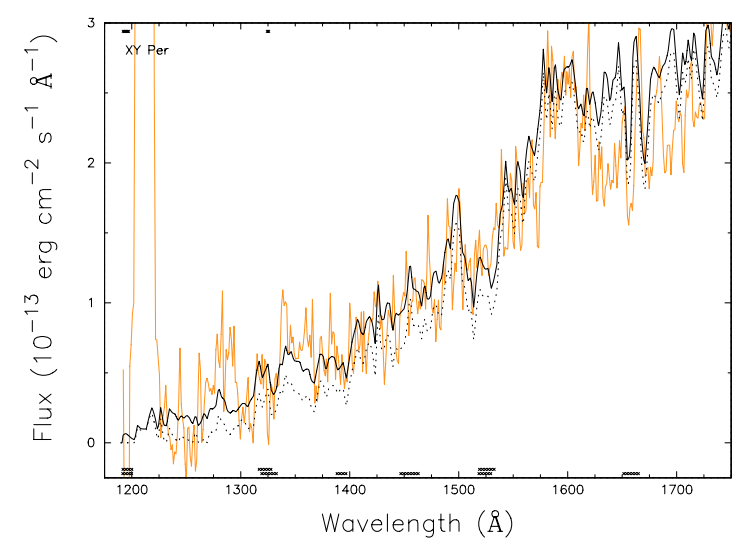

Fig. 61. XY Per A2 III.

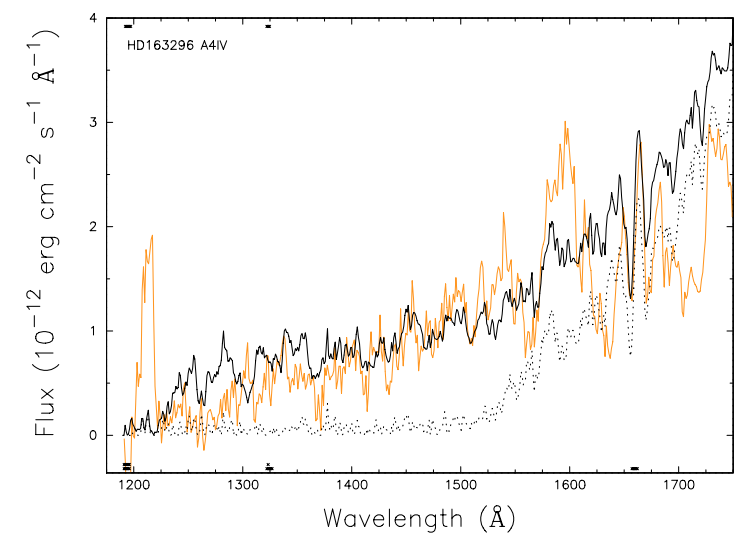

Fig. 62. HD 163296 A4 IV.

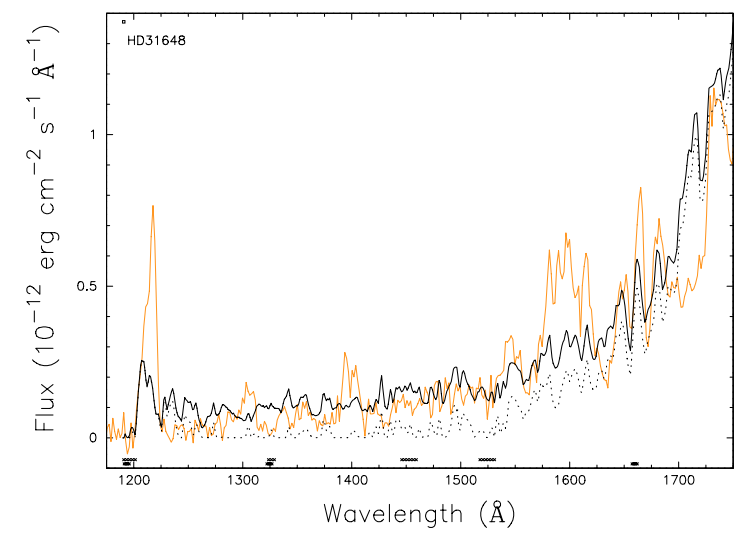

Fig. 63. HD 31648 A5 vz.

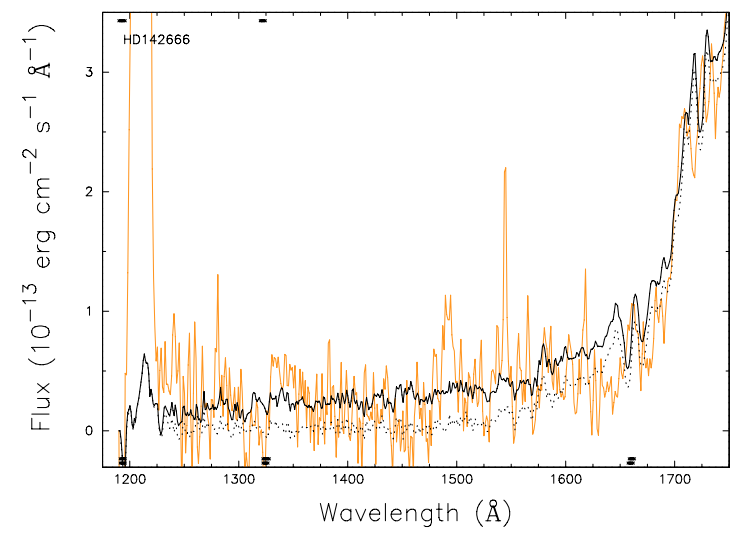

Fig. 64. HD 142666 A7 III.

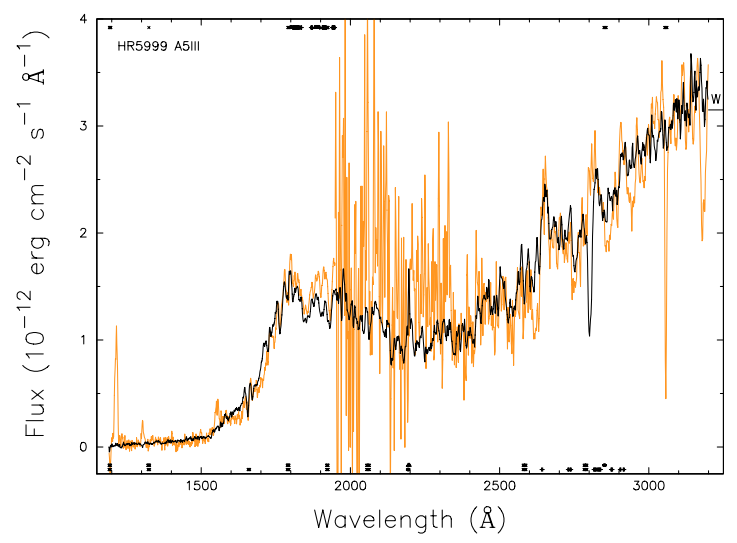

Fig. 65. HR 5999 A5 III.

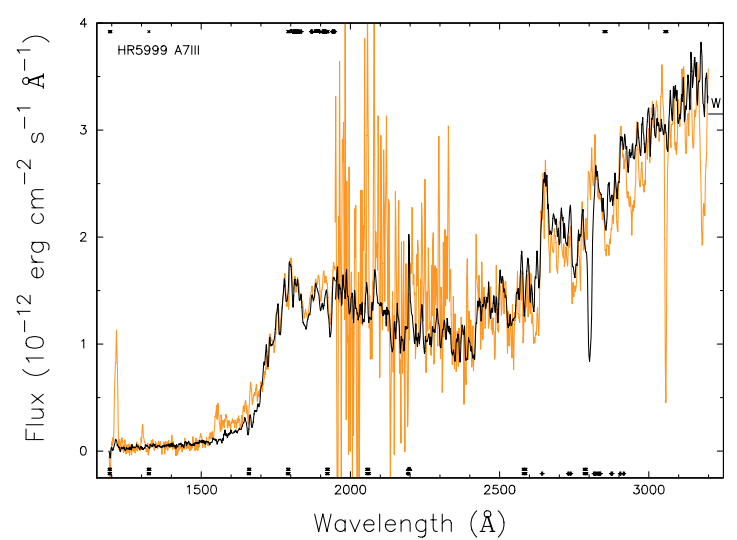

Fig. 66. HR 5999 A7 III.

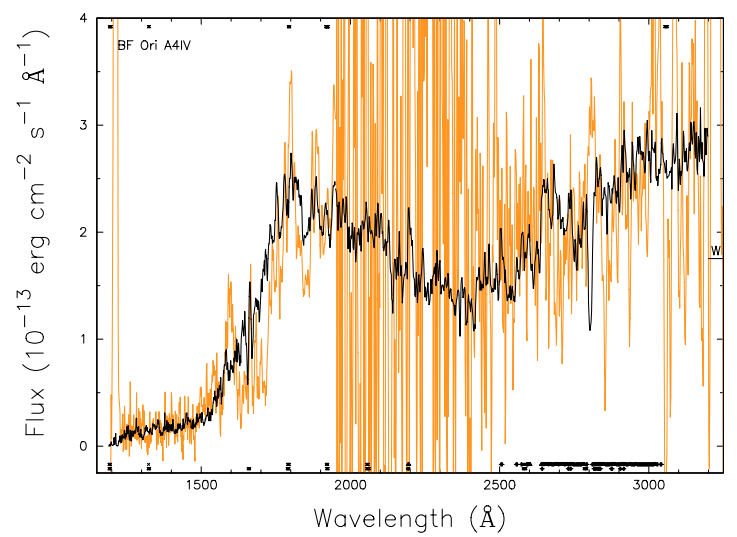

Fig. 67. BF Ori (07-02) A4 IV.

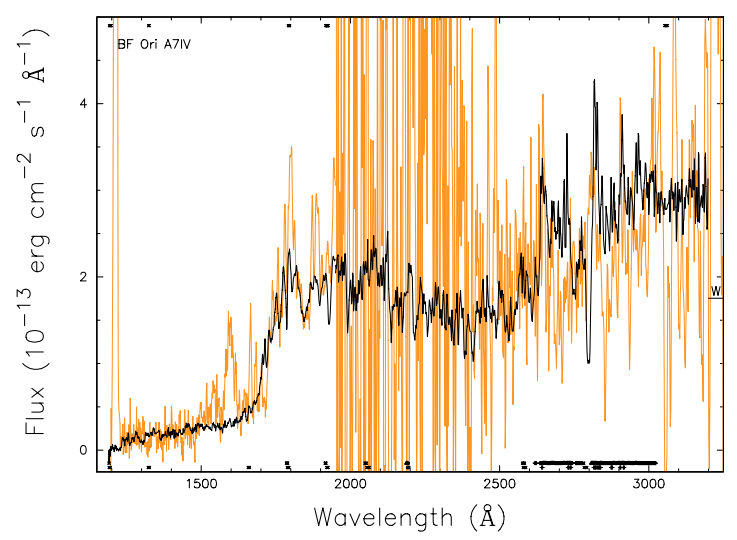

Fig. 68. BF Ori (07-02) A7 IV. 
P. F. C. Blondel and H. R. E. Tjin A Djie: Modeling of PMS Ae/Fe stars using UV spectra, Online Material p 9

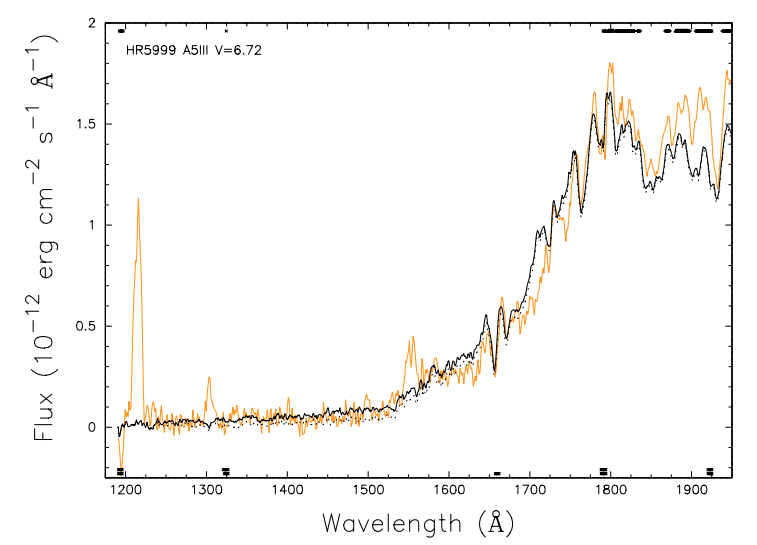

Fig. 69. HR 5999 A5 III.

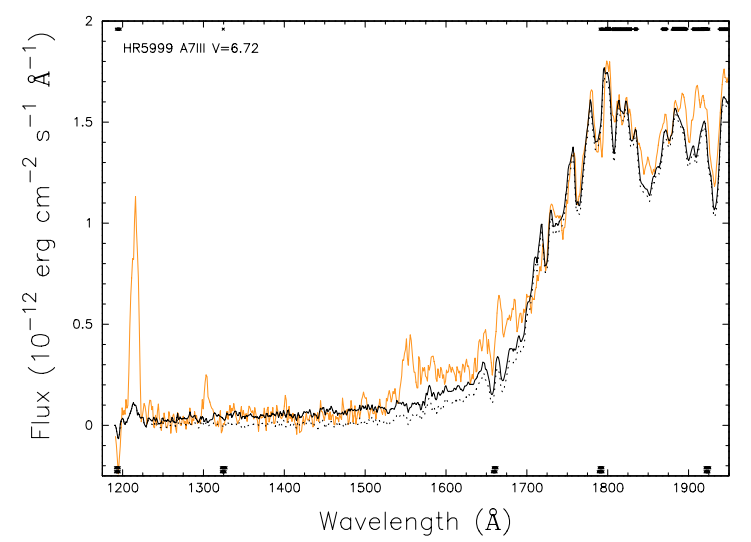

Fig. 70. HR 5999 A7 III.

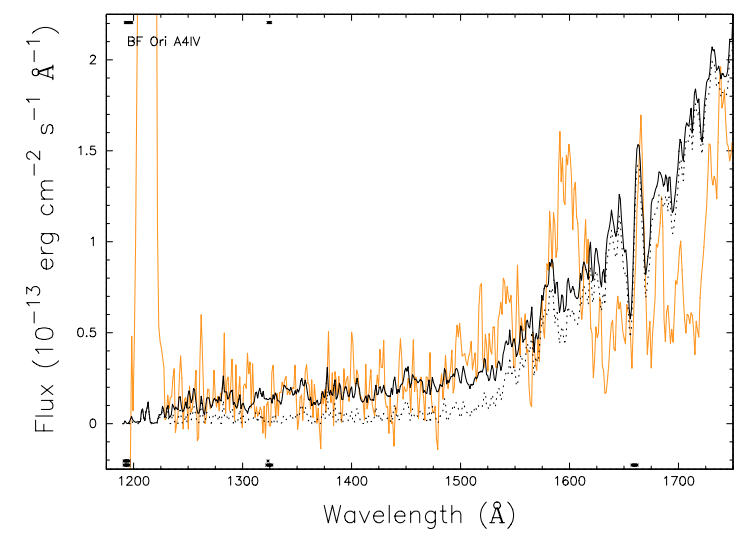

Fig. 71. BF Ori (07-02) A4 IV.

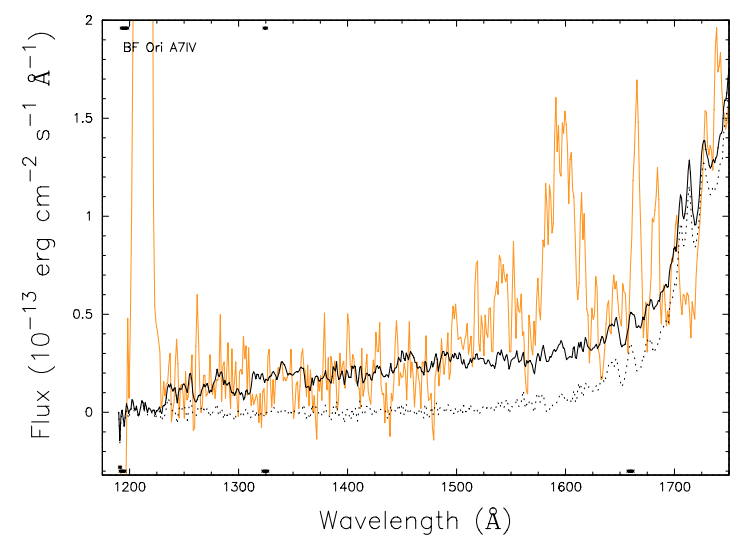

Fig. 72. BF Ori (07-02) A7 IV.

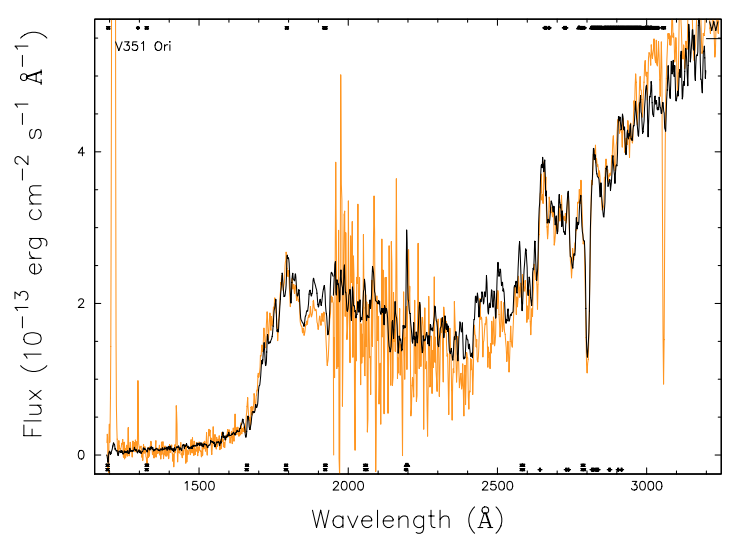

Fig. 73. V351 Ori A7 III.

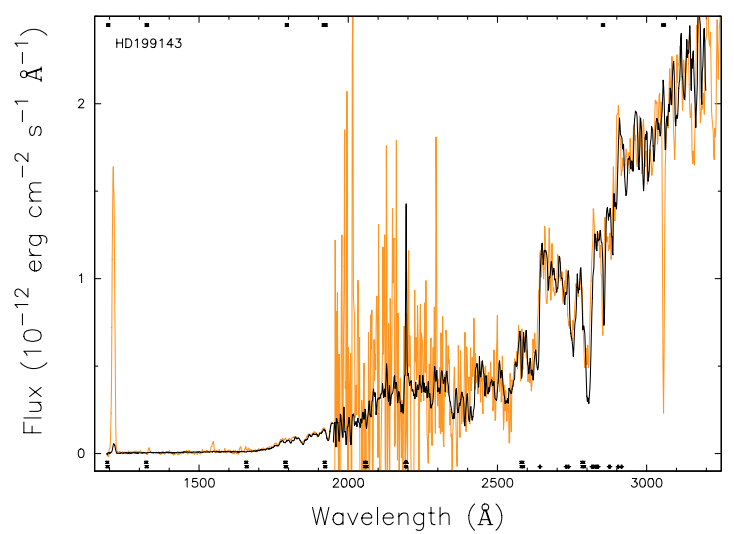

Fig. 74. HD 199143 F6 V.

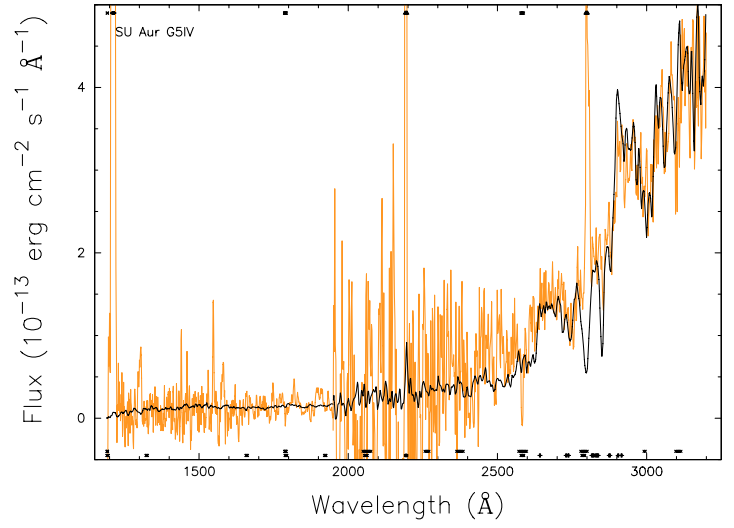

Fig. 75. SU Aur G5 IV.

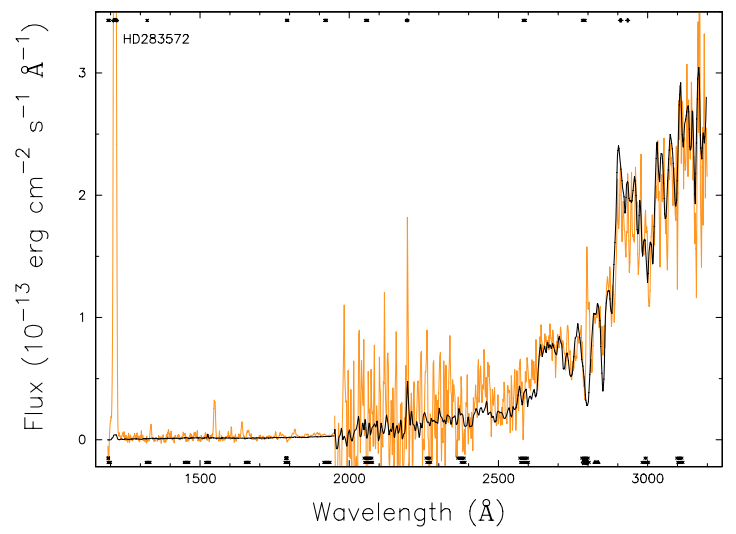

Fig. 76. HD 283572 G5 IV. 
P. F. C. Blondel and H. R. E. Tjin A Djie: Modeling of PMS Ae/Fe stars using UV spectra, Online Material p 10

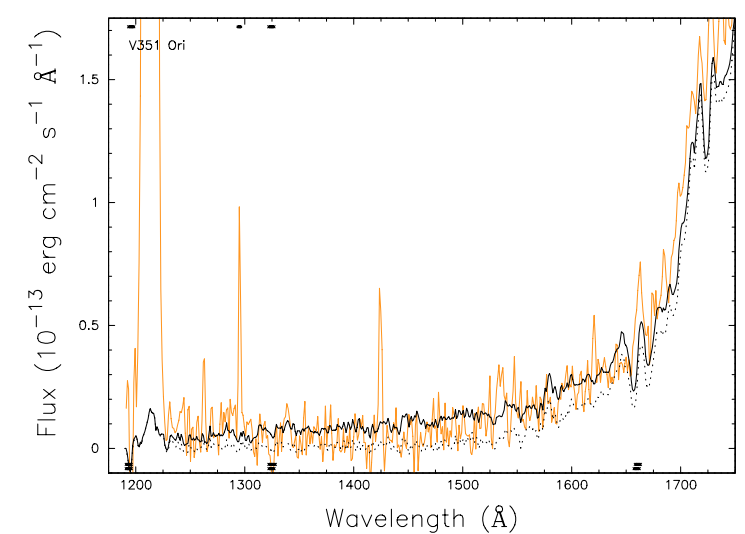

Fig. 77. V351 Ori A7 III.

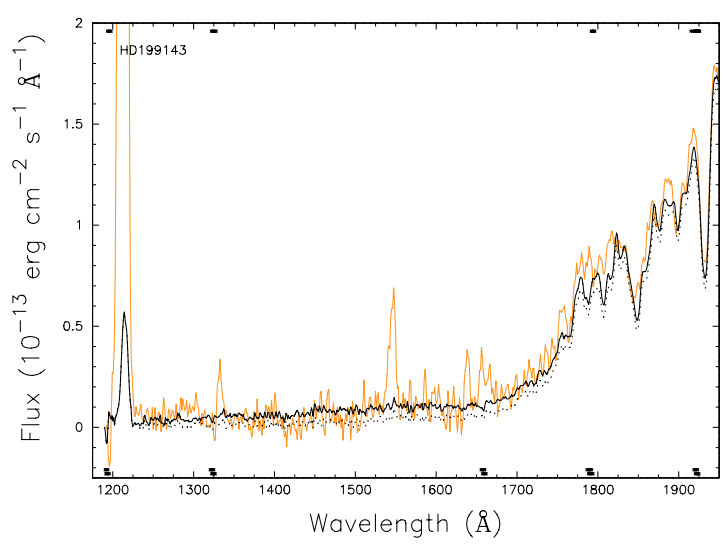

Fig. 78. HD 199143 F6 V.

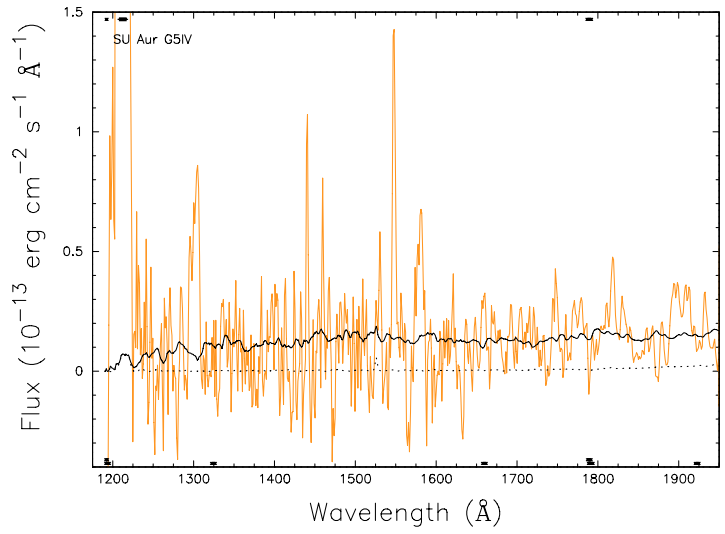

Fig. 79. SU Aur G5 IV.

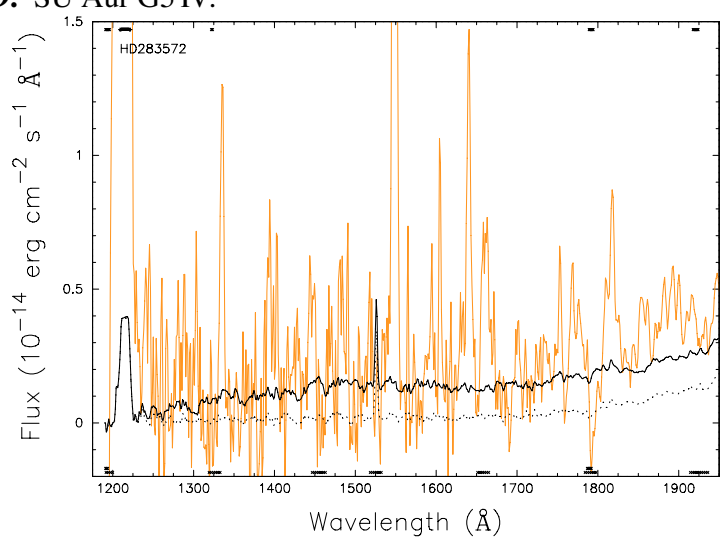

Fig. 80. HD 283572 G5 IV.

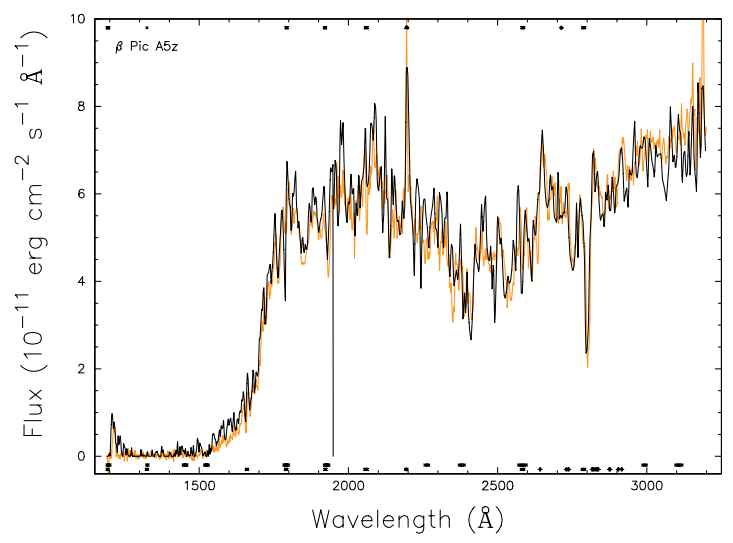

Fig. 81. $\beta$ Pic A5 Vz.

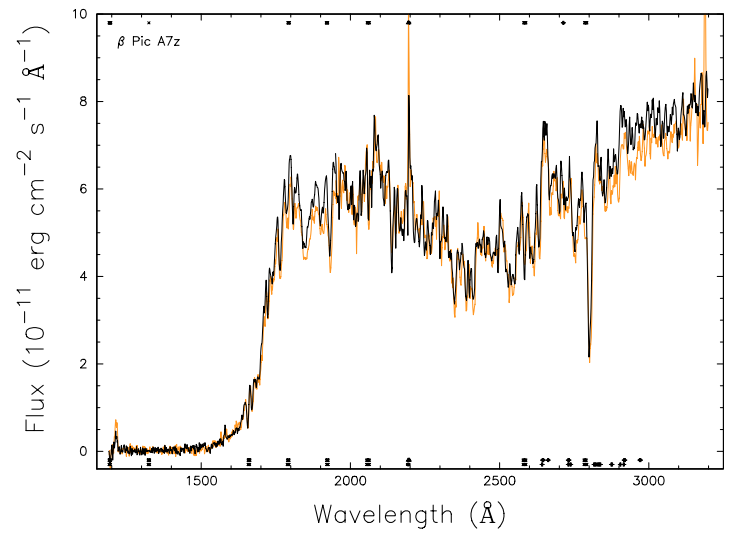

Fig. 82. $\beta$ Pic A7 Vz.

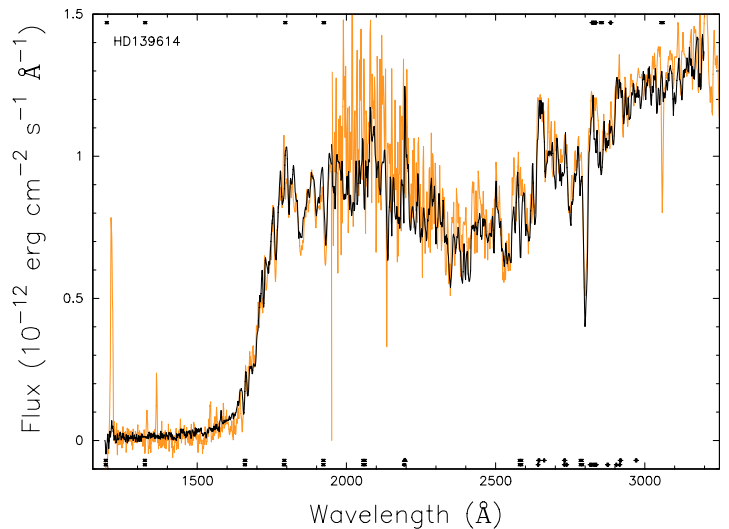

Fig. 83. HD 139614 A7 Vz.

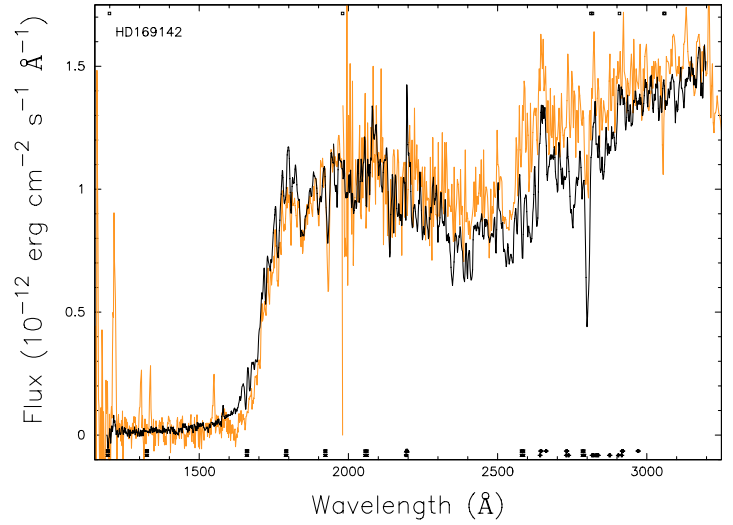

Fig. 84. HD 169142 A7 Vz. 
P. F. C. Blondel and H. R. E. Tjin A Djie: Modeling of PMS Ae/Fe stars using UV spectra, Online Material $p 11$

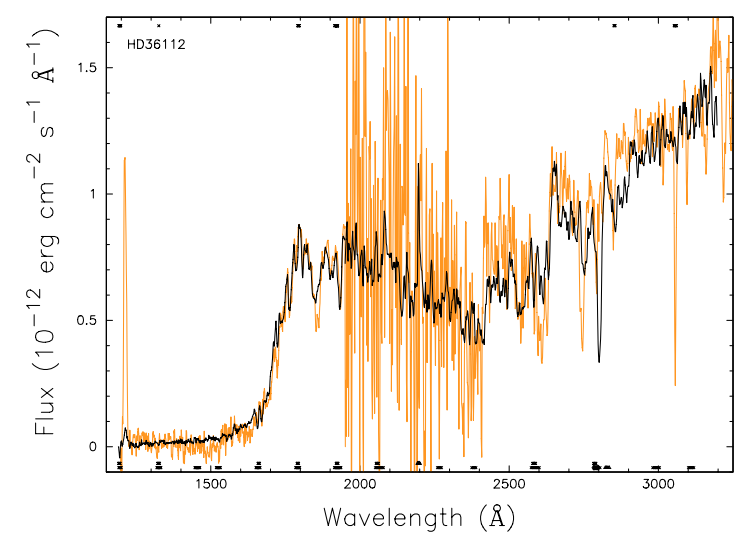

Fig. 85. HD 36112 A7 III.

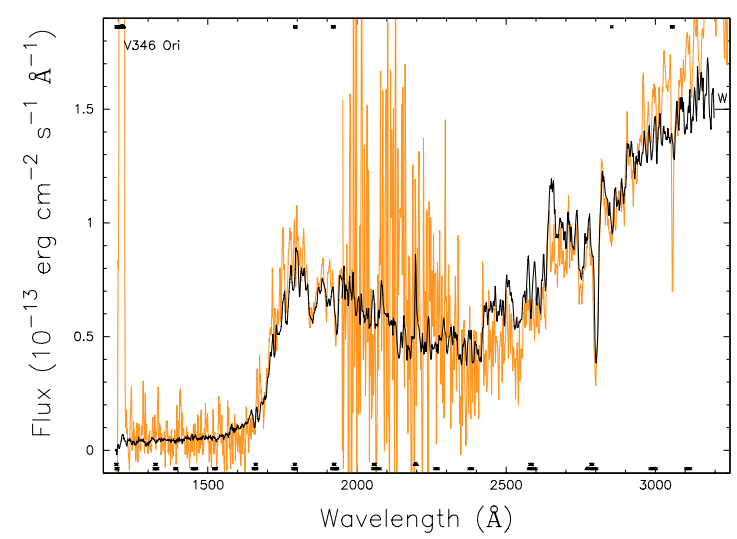

Fig. 86. V346 Ori A7 III.

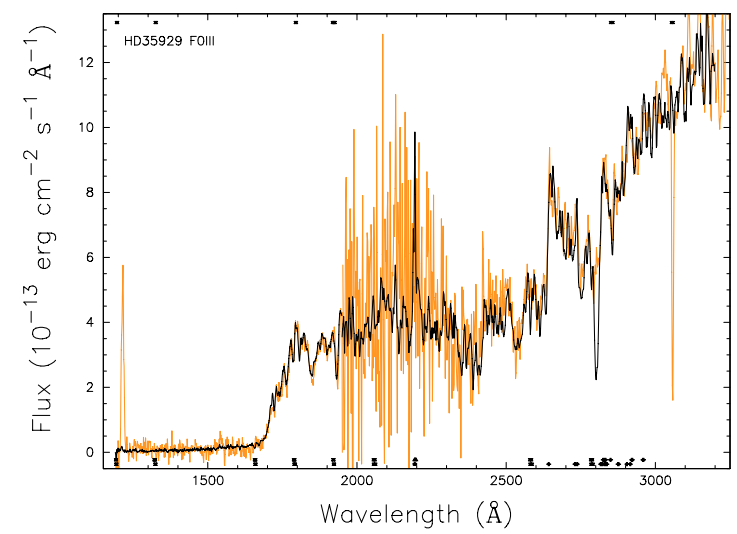

Fig. 87. HD 35929 F0 III.

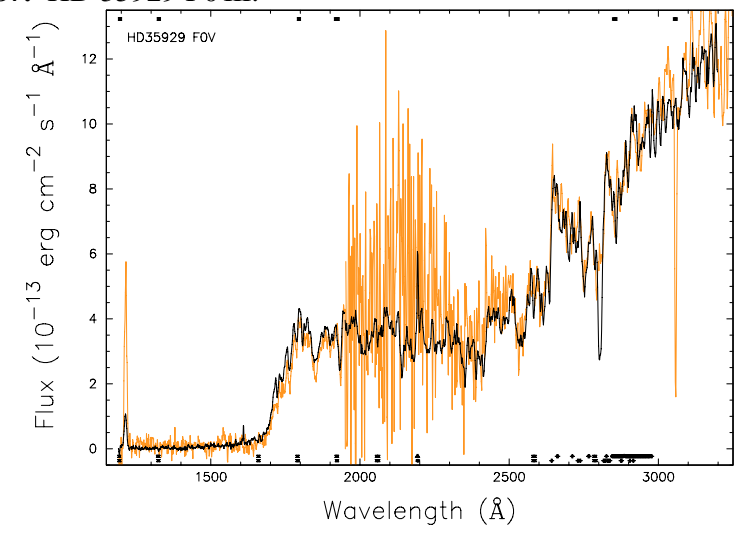

Fig. 88. HD 35929 F0 V.

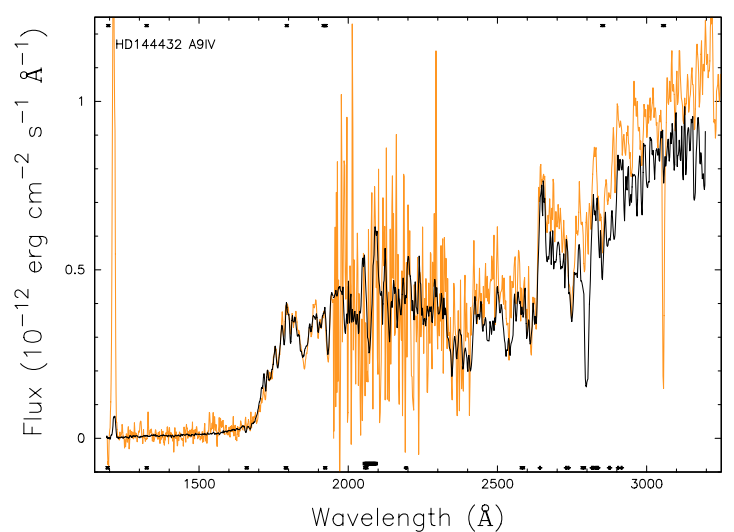

Fig. 89. HD 144432 A9 IV.

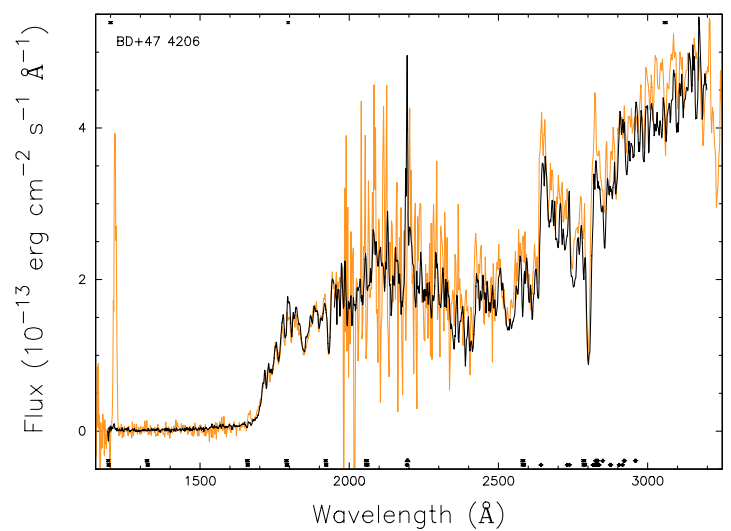

Fig. 90. $\mathrm{BD}+47^{\circ} 4206 \mathrm{~F} 0$ III.

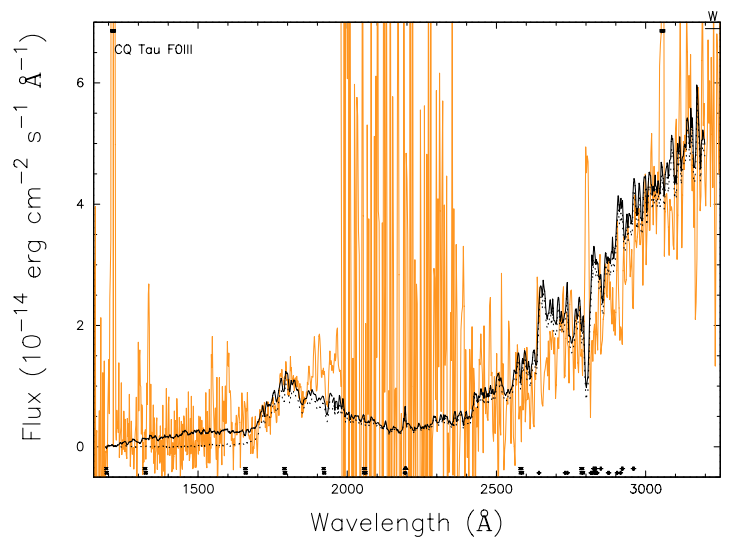

Fig. 91. CQ Tau F0 III.

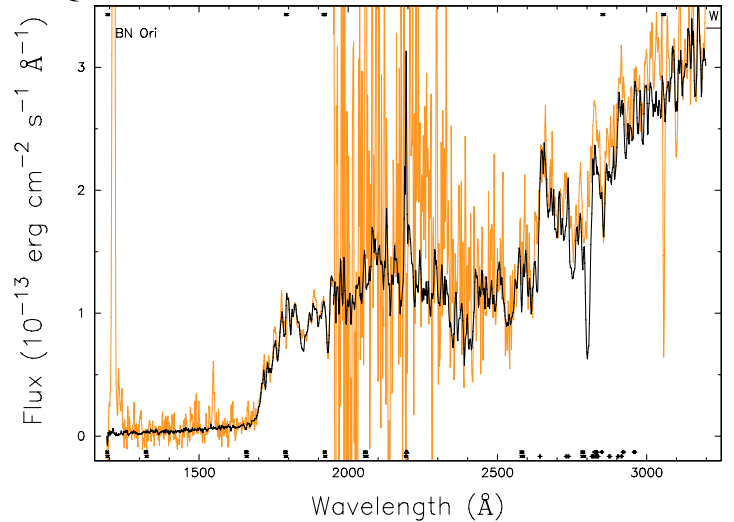

Fig. 92. BN Ori F0 III. 
P. F. C. Blondel and H. R. E. Tjin A Djie: Modeling of PMS Ae/Fe stars using UV spectra, Online Material p 12

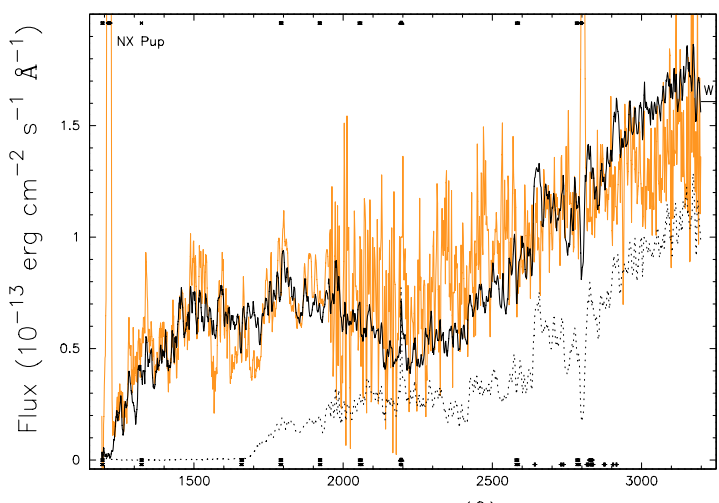

Fig. 93. NX Pup F2 III.

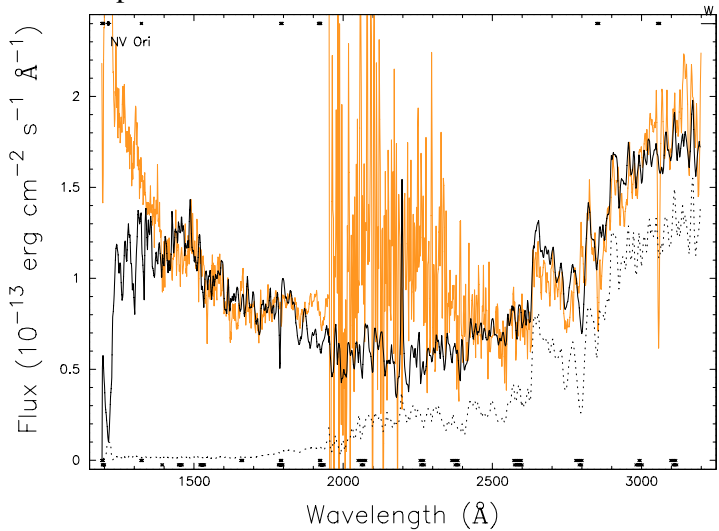

Fig. 94. NV Ori F6 III.

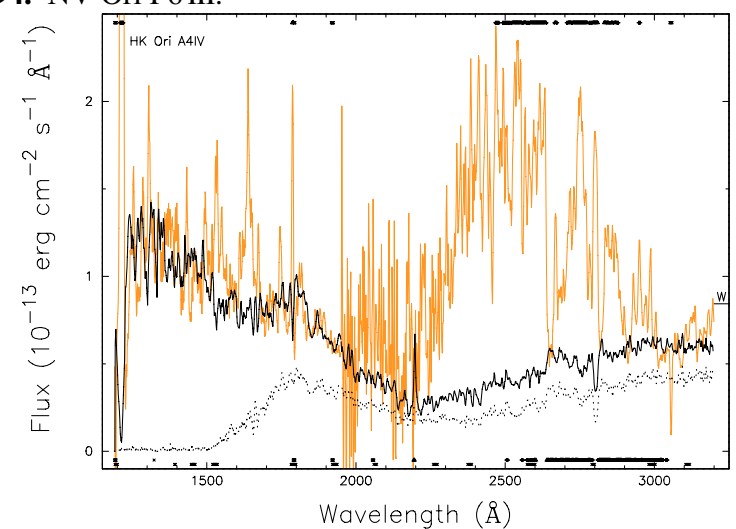

Fig. 95. HK Ori A4 IV.

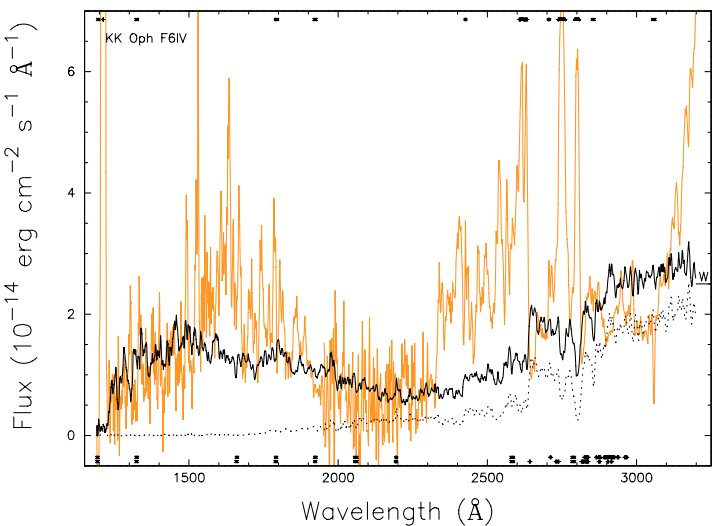

Fig. 96. KK Oph F6 IV.

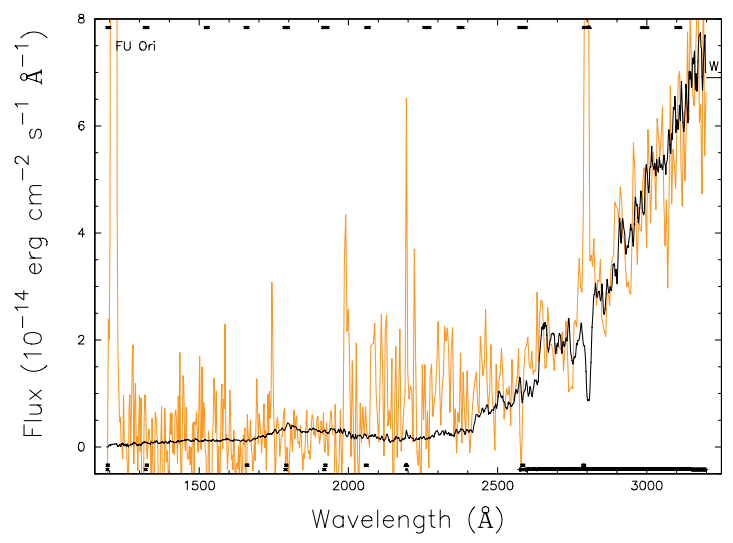

Fig. 97. FU Ori F0 I.

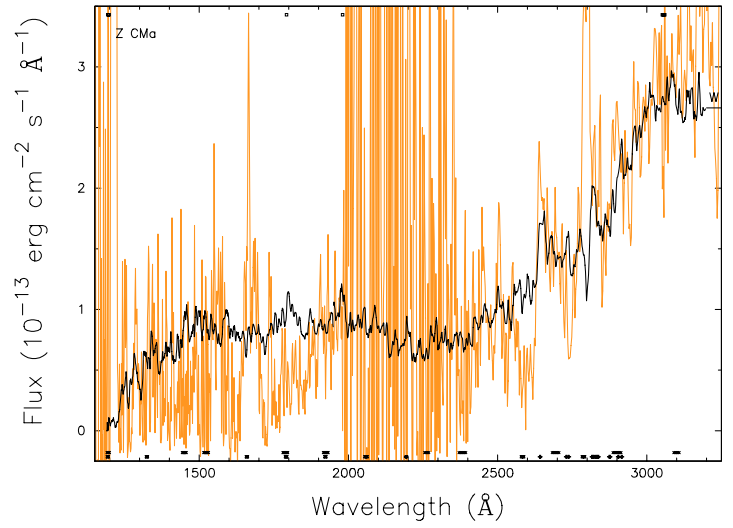

Fig. 98. Z CMa F2 I.

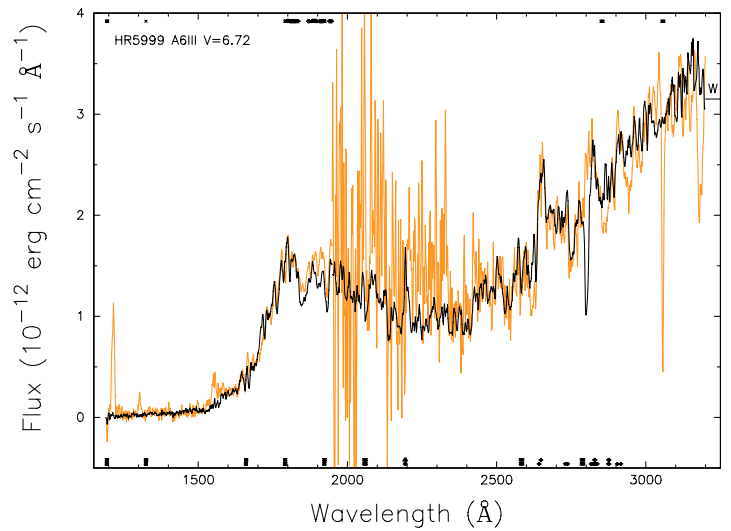

Fig. 99. HR 5999 A6 III\#1.

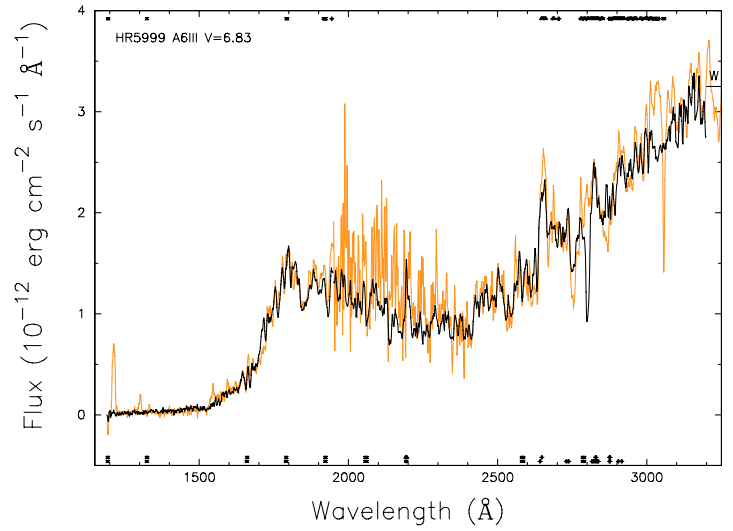

Fig. 100. HR 5999 A6 III\#2. 
P. F. C. Blondel and H. R. E. Tjin A Djie: Modeling of PMS Ae/Fe stars using UV spectra, Online Material $p 13$

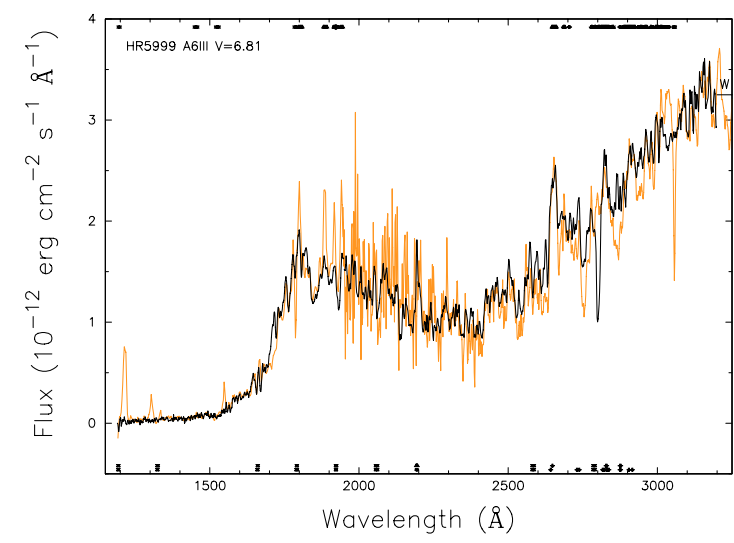

Fig. 101. HR 5999 A6 III\#3.

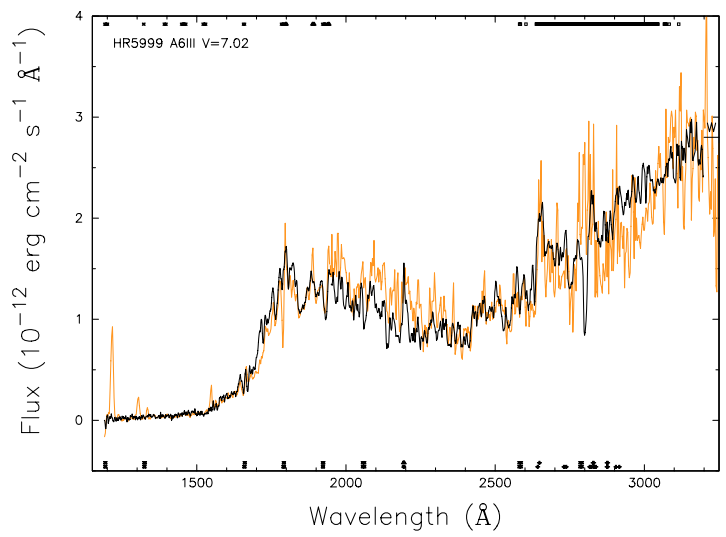

Fig. 102. HR 5999 A6 III\#4.

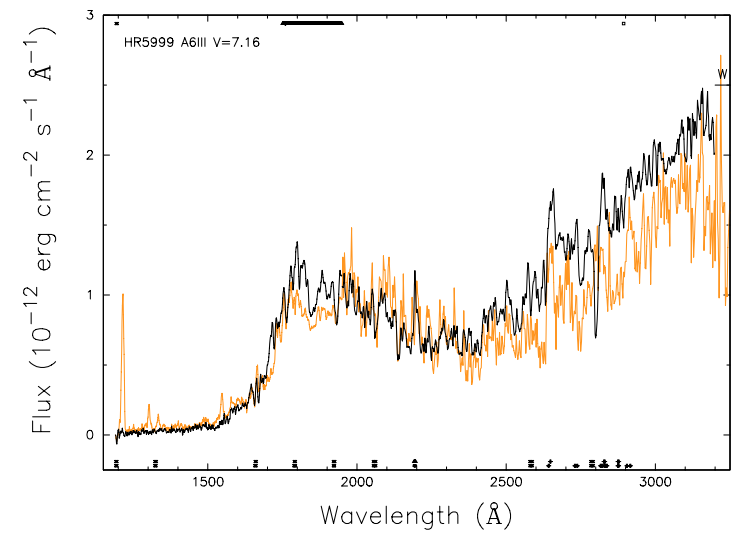

Fig. 103. HR 5999 A6 III\#5.

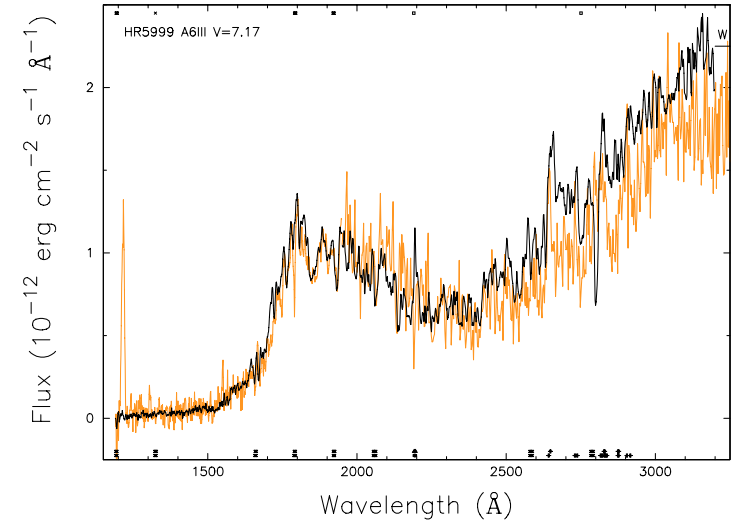

Fig. 104. HR 5999 A6 III\#6.

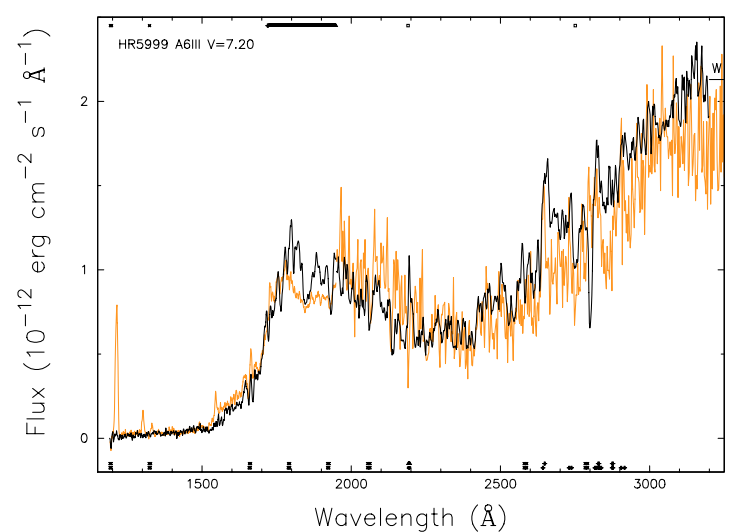

Fig. 105. HR 5999 A6 III\#7.

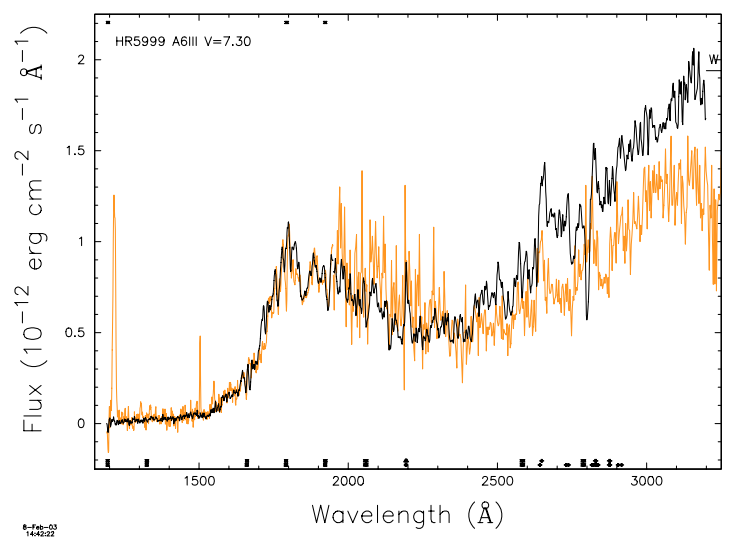

Fig. 106. HR 5999 A6 III\#8.

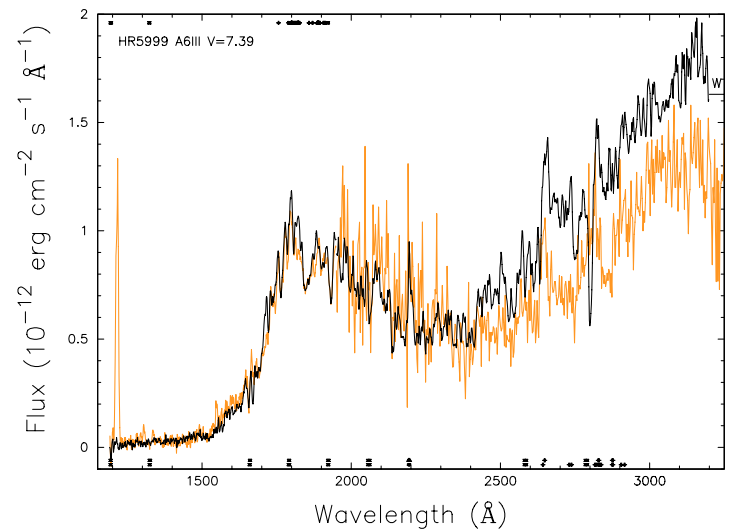

Fig. 107. HR 5999 A6 III\#9.

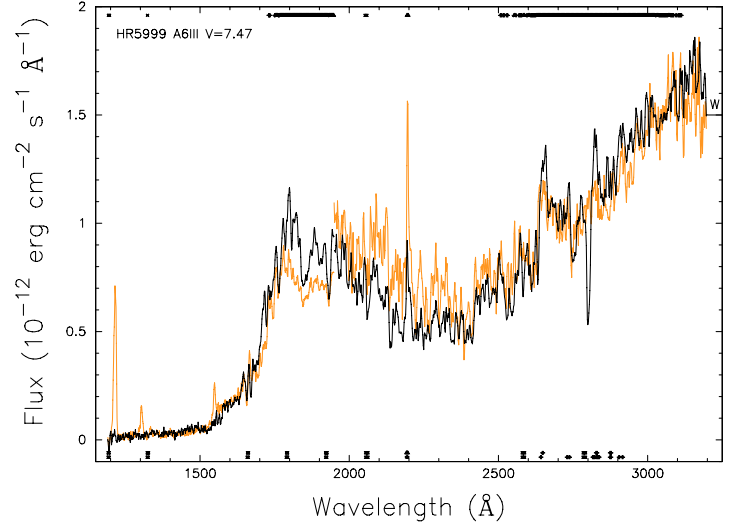

Fig. 108. HR 5999 A6 III\#10. 
P. F. C. Blondel and H. R. E. Tjin A Djie: Modeling of PMS Ae/Fe stars using UV spectra, Online Material p 14

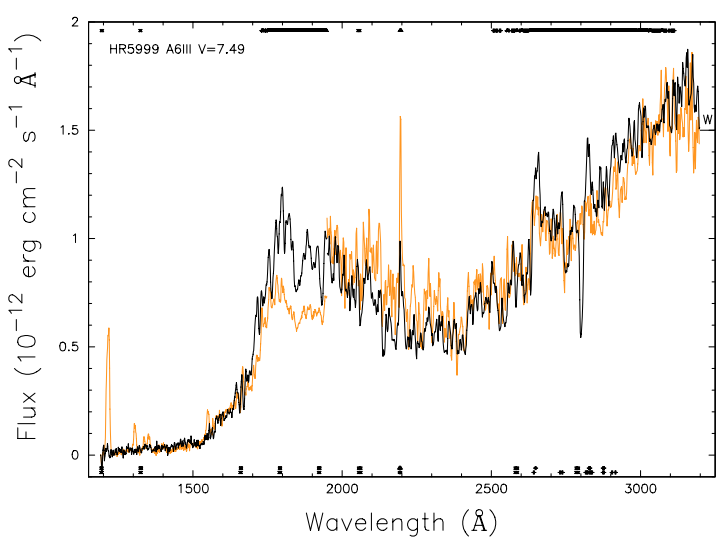

Fig. 109. HR 5999 A6 III\#11.

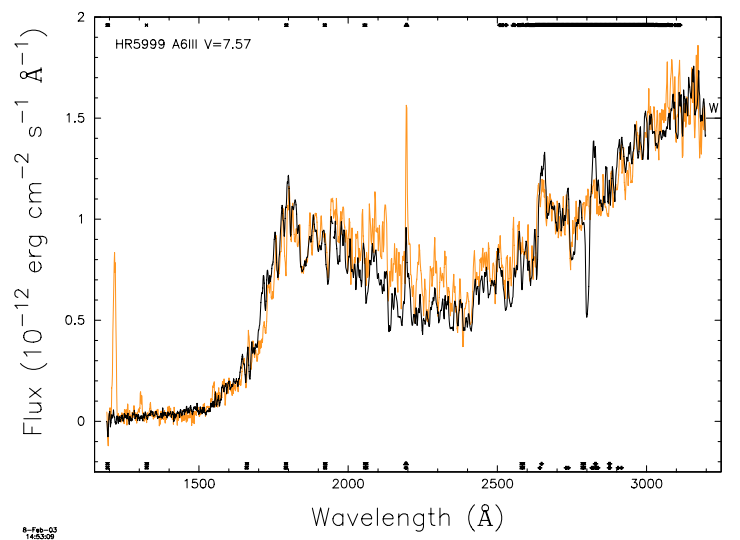

Fig. 110. HR 5999 A6 III\#12. 


\section{Appendix A: Justification of the UV3C-model}

In this section we give some arguments that justify our choice of the employed model considering that several alternative interpretations of the UV emission/excess (UVE) can be considered.

\section{A.1. Presence of a companion}

Although several of the discussed objects are known to be double stars, the UVEs that appear when the observed spectrum is compared with a standard star of the same -visual- spectral type have spectral shapes that correspond to a supergiant spectrum. The brightness, however, is far less than what would be expected from a supergiant at the distance of the observed stellar system. The same argument holds for the cases in which a giant spectrum was used.

\section{A.2. Hot surface spots}

Direct evidence of the presence of hot accretion spots at the stellar surface has been found for the cTT DI Cep (Gómez de Castro \& Fernández 1996). This interpretation is based on the correlation between the variation in the UV continuum and emissionline flux (in the SW-part of IUE) and on the simultaneous variation in the brightness $\left(\Delta V^{\mathrm{J}}=0^{\mathrm{m}}\right.$. 2$)$ with $P \sim 11^{\mathrm{d}}$. (Fernández \& Eiroa 1995).

In our sample of PMS Ae/Fe stars, we have only one star, HD 163296 (A3 V), which has been monitored in the SW-part of IUE with sufficient time-resolution to test the possibility of a hot surface spot. The stellar rotation period $\left(P_{\text {rot }}\right)$ can be estimated from $v \sin i=120_{-30}^{+20} \mathrm{~km} \mathrm{~s}^{-1}(\mathrm{Fz} 85)$ and $R_{\star}=1.76 R_{\odot}$ (Table 2). For $i=57^{\circ}$, this results in $P_{\text {rot }}=13.91_{-2.00}^{+4.63}$.

Two sequences of UV spectra have been obtained ${ }^{8}$ in 1986 and 1987 (Table F.5). During these campaigns the FES camera operated well and could be used for photometric monitoring with an accuracy of \pm 0 . 05 in $V^{\mathrm{J}}$.

In spite of many attempts to detect periodicities in the $V^{\mathrm{J}}$-band fluxes and UV emission-line fluxes with both Fourier-methods and information-entropy minimalisation methods (Cincotta et al. 1995), no significant periodicity could be found. We were also not able no find any direct correlation between the flux variations in the UV and visual. The flux observed shortward of $1600 \AA$ varies irregularly by at most $30 \%$, but never disappears completely. Unless HD 163296 is seen "poleon" (which seems improbable in view of $v \sin i \approx 120 \mathrm{~km} \mathrm{~s}^{-1} \approx$ $\left.\frac{1}{4} v_{\mathrm{c}}=\frac{1}{4} \sqrt{G M_{\star} / R_{\star}}\right)$ the hot $\mathrm{UV}$ emission region has to be extended over an equatorial belt on the surface of the star or over the boundary-layer of an accretion disc. There are two possibilities for the existence of a hot belt at the stellar surface: magnetic accretion from the accretion disc to the star and "neutral" accretion that could occur for accretion through cool BLs. The presence of a hot magnetic belt has also been assumed to interpret the emission-line profiles of $\operatorname{Ly} \alpha$, as seen in the highresolution UV spectra of HD 163296, HR 5999, and HD 104237 (Appendix D.4). From these observations we derive that the emission belt could extend at least to latitudes of $35^{\circ}$ (Blondel et al. 1993). The observations of HD 163296 show variability but no indication of periodicity in the UV emission excess $\left(\delta_{\mathrm{bl}}\right)$, so that there is no evidence of spots that persist longer than 14 . . Similar variability was also found in the UVE of several other PMS Ae/Fe stars for which IUE observations were

8 In collaboration with dr. C. Catala, dr. F. Praderie \& dr. T. Simon (Catala et al. 1989). obtained at various dates, e.g. for HD 104237, NX Pup and AK Sco (Table 2), but these observations are too few and too widely separated in time to give any significant information about possible periodicity in $\delta_{\mathrm{bl}}$ or the presence of hot spots.

\section{A.3. Magnetic accretion}

In contrast to the situation for $\mathrm{T}$ Tauri stars, there are indications that large-scale magnetic fields do not play a dominant role in the accretion process for Herbig emission-line stars. This is suggested by the observation that the rotation velocities of PMS Be/Ae/Fe stars are larger than those observed for T Tauri stars by a factor of 2.0-4.5 (Table 1). The rotation velocities of T Tauri stars are in fact lower than expected from their evolutionary contraction process and their accretion phases, which both tend to spin these stars up during their PMS contraction. The general explanation for the low rotation of the T Tauri stars is the braking effect due to the coupling of the stellar magnetic field with the toroidal component of the disc magnetic field outside the co-rotation radius (Cameron et al. 1993, 1995; Yi 1994; Armitage \& Clarke 1996). Since the co-rotation radii of Herbig stars with masses around $2 M_{\odot}$ are close to those of T Tauri stars $\left(\sim 1 M_{\odot}\right)$ and both their contraction rates (PS93) and mass accretion rates are of the same order as those of the T Tauri stars, the difference in braking of the rotation can only be explained by the relative weakness of the large-scale stellar magnetic field of the Herbig stars. For stellar dynamo fields, this may perhaps be explained by the fact that, in contrast to the fully convective Hayashi phases of $\mathrm{T}$ Tauri stars, convection in more massive stars is limited to the surface layers during the first contraction phases. However, we cannot exclude the possibility that small-scale dynamo or fossile stellar fields may be responsible for magnetic accretion in a non-uniform activity belt at the stellar surface. Such fields certainly play a role in supporting the (bipolar) outflow from the BL, which is revealed by intense line emission (Paper II). Instead of trying to model both accretion and outflow, we prefer to limit ourselves here to the accretion proces, in particular, to the well-studied models of "neutral" (nonmagnetic) accretion.

\section{A.4. "Neutral" accretion}

Since there are no indications that magnetic fields play a dominant role in the process of accretion on PMS Ae/Fe stars (except perhaps in controlling the outflow perpendicular to the disc plane), we feel justified in comparing the global BL parameters from our UV3C-model analysis with the predictions of several self-consistent BL models for neutral accretion. These models are based on the simultaneous solution of the equations of mass-, radial momentum-, angular momentum-, and energyconservation, together with the boundary conditions for the inner and outer boundaries of the BL. Some supplementory assumptions concerning the viscosity in the disc, the equation of state, the opacity law, the rotation rate, and the mass outflow rate have to be made as well.

Several 1-D stationary models with axial symmetry have been explored for $1 M_{\odot}$ stars. These self-consistent models have in common that for $\dot{M} \approx 10^{-7} M_{\odot} \mathrm{yr}^{-1}$ the structure of the BL can be separated into two disinct regions: a narrow $\left(\Delta r \sim 0.01 R_{\star}\right)$ dynamical region around the star where the Kepler rotation of the disc is braked down to the stellar rotation and, around this zone, a broader $\left(\Delta r \sim 0.1-0.2 R_{\star}\right)$ and cooler thermal region, where this viscous braking energy is dissipated by radiation. 
The density- and temperature-structures of the BL strongly depend on the choice of the inner boundary condition (Regev \& Bertout 1995). If the radiative flux at the inner boundary (or stellar surface) is negligible and the viscosity parameter $\alpha \approx 0.1$, the resulting BL will be hot $(8000-10000 \mathrm{~K})$ and narrow $(\delta \approx$ $\left.0.02 R_{\star}\right)$. Such results were obtained by Popham et al. (1993) under the assumption of optically-thick BL radiation. However, if the inner boundary is required to have the surface temperature of the star (Bertout \& Regev 1992; Lioure \& Le Contel 1994) a significant part (10-30\%) of the BL energy is radiated inward into the outer layers of the star and creates a hot $(T \approx 10000 \mathrm{~K})$ equatorial belt. Godon (1996) repeated these model calculations with improved opacities in order to account for the ionised and neutral regions in the $\mathrm{BL}$ and the disc. For small values of $\alpha(\approx 0.01)$ he finds cool $(5000-6000 \mathrm{~K})$ and optically-thick $(\tau \approx 1-10) \mathrm{BL}$ 's, in agreement with earlier results, but his thermal BL is broader $\left(\delta \approx 0.2 R_{\star}\right)$. For higher values of $\alpha(0.1-1.0)$ the BL radiation becomes optically thin $(\tau \lesssim 1)$, which corresponds to less efficient cooling, and its temperature may rise to $8000-10000 \mathrm{~K}$. These temperatures are similar to those obtained by Basri \& Bertout (1989) for the optically-thin approximation that they needed to fit the Balmer continuum and line emission. However, Godon (1996) finds larger thermal widths (up to $0.04 R_{\star}$ ) than these authors.

Later Kley \& Lin (1996) also calculated the BL structure with a $2 \mathrm{D}$ axial symmetric, non-stationary model for a $1 M_{\odot}$ star with a rotation rate of 0.1 of the brake-up rate. For three representative mass accretion rates $\left(10^{-7}, 10^{-6}, 10^{-4} M_{\odot} \mathrm{yr}^{-1}\right)$ and $\alpha=0.01$, the time evolution of the main physical parameters (temperature, density, pressure, and angular momentum) was followed in and perpendicular to the disc plane. The final models are consistent with the results of the 1D stationary models. For the case of the lowest $\dot{M}$, there is some heating of the stellar atmosphere over latitudes up to $5-10^{\circ}$, and for increasing accretion rates, the width of the heated surface zone increases via $30^{\circ}$ up to the whole stellar surface. Although there is general agreement between the 2D and 1D models, the numerical values of the parameters in the equatorial plane are somewhat different.

It should be noted here that the hot component that is present in the UV spectra of the PMS Ae/Fe stars may be interpreted equally well by the radiation from a hot equatorial belt on the stellar surface, as predicted by the models of Regev \& Bertout (1995) and Kley \& Lin (1996). An analysis in terms of such a model would be similar to the analysis employed in this paper.

\section{A.5. Interpretation of the UV-spectra}

In most of our sample spectra (for spectral types later than A2), we observed a residual spectral component shortward of $1800 \AA$, which is not present in the photospheric spectra of the comparison stars of the same spectral type, given in the IUE spectral atlases. In this paper we have interpreted this component as the spectral contribution of a hot BL formed in neutral disc accretion to the star. This interpretation is based on the properties of the residual component, which show up most clearly in late-type stars, such as NX Pup, V380 Ori, HK Ori, and the early-type star $\mathrm{BD}+46^{\circ} 3471$. We observed two properties of this spectral component:

(1) The (BL) continuum in the UV can be matched very well with that of a late-B or early-A type supergiant (e.g. $\sigma$ Cyg - B9 Iab), corresponding to a Planck spectrum of $T_{\text {eff }} \approx 10000 \mathrm{~K}$. The optical depth in the UV wavelength range $(1200-3000 \AA)$ is $\sim 1$, and if we extrapolate the resid-

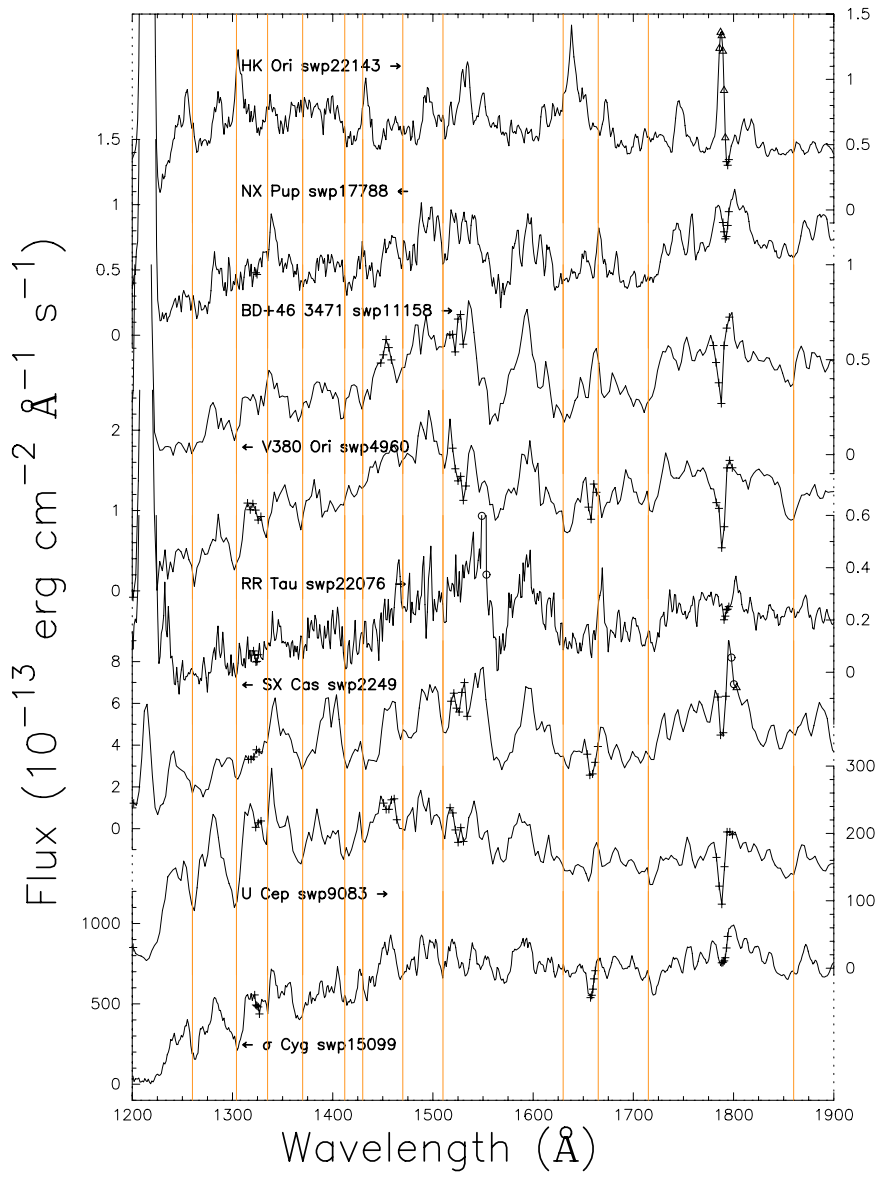

Fig. A.1. Comparison of several low-resolution UV spectra. The arrows indicate the $y$-axis of the spectrum. See Table A.1 for an identification of the marked lines.

ual BL spectrum, we find $\tau \approx 3.4$ around $\lambda=3600 \AA$ (where the flux of the supergiant reaches its maximum). We therefore consider the UV spectrum of the BL in Herbig stars as optically-thick, in agreement with expectations from theoretical models of BL, as mentioned in Sect. A.4.

(2) The SW-part of the spectra of the (BL) component (Fig. A.1) are characterised by strong and broad absorption features, similar to those observed in the spectra of B9 I-A2 I type stars, as noted earlier in the SW-part of the spectrum of NX Pup by Tjin ADjie et al. (1984). A list of these absorption features with probable identifications is given in Table A. 1 for the five stars. These features are sharpest in the spectra of BD+46 3471 and V380 Ori and broader (partly merged with neighbouring lines due to turbulence) in that of NX Pup. They probably are blends of the strong resonance lines of Si II, Si III, Al II, Al III, Fe II, and perhaps Si IV, as is expected above a hot region of outflow. The features observed in the Herbig stars are deeper than those observed in the supergiants, which may indicate a higher column density. The blended features in the spectrum of NX Pup are at least as broad as those observed in the supergiants, which suggests a strong degree of turbulence in the region of line formation. This suggestion has to be checked by high-resolution spectroscopy. The characterisation of the line-formation domain as a dense, hot, and probably turbulent region suggests that this region is located immediately outside the continuum source, which we interpret as the boundary-layer. 
Table A.1. Absorption features in the UV spectra (Fig. A.1) of some PMS Ae/Fe stars, SX Cas, U Cep and $\sigma$ Cyg. $(\mathrm{n}=$ narrow, $\mathrm{b}=$ broad, $\mathrm{d}=$ deep, $\mathrm{s}=$ shallow, $\mathrm{e}=$ emission instead of absorption, $:=$ uncertain value.)

\begin{tabular}{|c|c|c|c|c|c|c|c|c|c|}
\hline$\overline{\bar{\lambda}}$ & 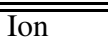 & $\sigma$ & $\overline{\overline{V 380}}$ & $\overline{+46^{\circ}}$ & $\overline{\mathrm{NX}}$ & $\overline{\overline{\mathrm{HK}}}$ & 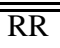 & 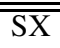 & $\overline{\overline{\mathrm{U}}}$ \\
\hline$(\AA)$ & - & Cyg & Ori & 3471 & Pup & Ori & Tau & Cas & Cep \\
\hline 1260 & $\begin{array}{l}\text { Si II } \\
+ \text { S I }\end{array}$ & nd & $\mathrm{b}$ & $\mathrm{b}$ & $\mathrm{b}$ & $\mathrm{b}$ & nd & $\mathrm{b}$ & nd \\
\hline 1304 & $\begin{array}{l}\text { O I } \\
+ \text { Si II }\end{array}$ & bd & bd & bd & bs & e & bd & bd & bd \\
\hline 1335 & C II & e: & e: & e: & $\mathrm{e}$ & - & e: & e: & e: \\
\hline 1370 & Si III & bd & nd & bs & bs & - & bs & bd & bd \\
\hline 1412 & Si III & bd & s & bs & nd & bd & nd & bd & bd \\
\hline 1430 & $\begin{array}{l}\text { C III } \\
+S_{I}\end{array}$ & nd & bs & b & $\mathrm{n}$ & $\mathrm{e}$ & $\mathrm{n}$ & bd & nd \\
\hline 1470 & $\begin{array}{l}\text { Si II } \\
+ \text { Fe II }\end{array}$ & bd & bs & bd & bs & bs & nd & bd & bs \\
\hline 1510 & $\begin{array}{l}\text { Si II } \\
+ \text { S II }\end{array}$ & $\mathrm{ns}$ & ns & bs & nd & ns & nd & nd & ns \\
\hline 1565 & $\begin{array}{l}\mathrm{Al} \text { II } \\
+\mathrm{Fe} I \mathrm{II}\end{array}$ & $\begin{array}{l}\text { bd } \\
+\mathrm{C}_{\mathrm{I}}\end{array}$ & bd & bd & bd & - & bd & bd & bd \\
\hline 1630 & $\begin{array}{l}\mathrm{Al} \mathrm{III} \\
+ \text { Mg I: }\end{array}$ & : & bd & bd & bd & $\mathrm{e}$ & bd & bd & bs \\
\hline 1715 & Al II: & bd & bd & bd & bd & bs & bd & bs & bd \\
\hline 1860 & $\begin{array}{l}\mathrm{Al} \text { III } \\
+ \text { Fe III }\end{array}$ & bd & bd & bd & bd & - & - & ns & bd \\
\hline
\end{tabular}

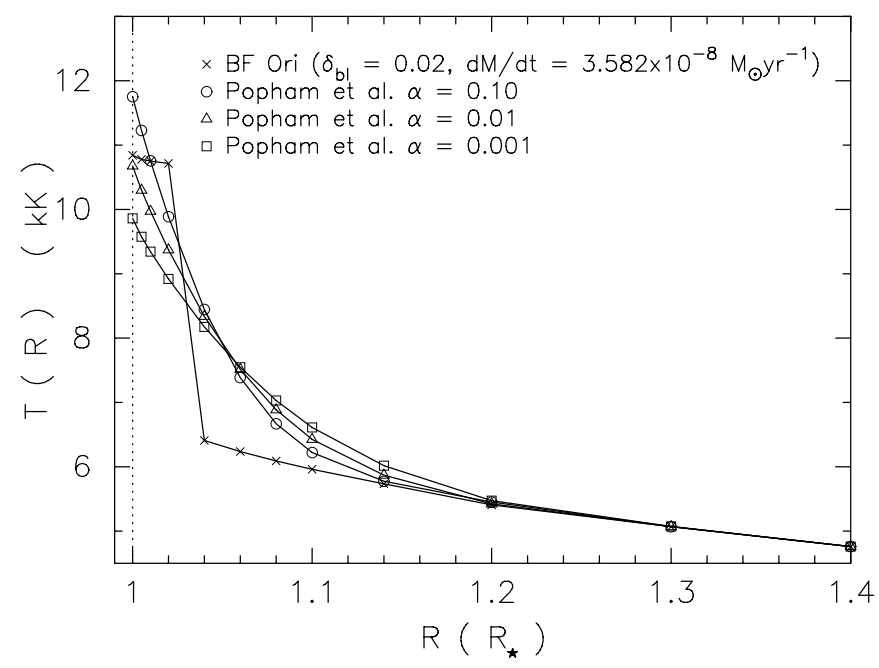

Fig. A.2. UV3C-model temperature distribution for BF Ori compared with the radial temperature distribution of the 1D BL model by Popham et al. (1993), both calculated for $\delta_{\mathrm{bl}}=0.02$. (see Table $2(7 \mathrm{p})$ for the BF Ori parameters).

We should note here that similar features have been observed in the spectra, taken outside eclipse, of SX Cas and U Cep (Plavec et al. 1982; Plavec 1983). These W Serpentis stars are eclipsing binaries or cataclysmic variables (CVs) consisting of a B7 star and a late-type giant in a phase of mass transfer. The UV continua have been explained in terms of an accretion disc boundary-layer around the receiving Bstar. The gross observational properties could be understood if the BL is a strongly turbulent (Shore \& King 1986) region. During the eclipse of the B-star component in the CV, the UV continuum disappears and is superseded by a rich emission-line spectrum of high-ionisation lines. This is interpreted as the emission by the "chromosphere" of the BL which emerges when the BL itself is shielded by the eclipse. A similar

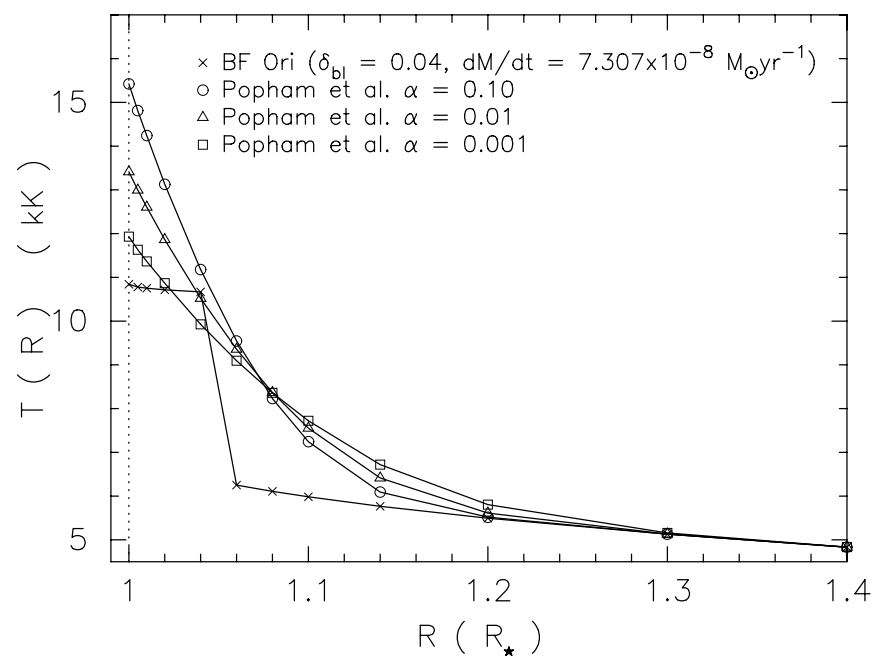

Fig. A.3. As previous figure but for $\delta_{\mathrm{bl}}=0.04$.

explanation can be given for the emission spectra of Herbig stars. In most of the UV spectra of the PMS Ae/Fe stars of our sample we observed strong emission-lines of O I, C II, C IV, Si II, Si III, Si IV, and N V emerging from the low continuum shortward of $1800 \AA$. We suggest that these lines are emitted outside of but close to the BL, which itself (similar to the CV case) is shielded by the disc when the star is observed "edge-on". A study of these emission regions will be presented in Paper II.

\section{A.6. The BL temperature distribution}

If the residual spectral component discussed above is indeed due to the $\mathrm{BL}$, our analysis with the UV3C model should yield $\delta_{\mathrm{bl}^{-}}$ values in the range of the values predicted by theoretical models for the thermal region of optically-thick boundary-layers. We compared the radial temperature distribution, corresponding to the $T_{\mathrm{eff}}, R_{\star}, M_{\star}, T_{\mathrm{bl}}$, and $\delta_{\mathrm{bl}}$ for the case of BF Ori, to the radial temperature distributions for a $1 M_{\odot}$ star, as predicted by the 1D model for different values of the viscosity parameter of Popham et al. (1993). Although we have not seen their results for $2 M_{\odot}$ stars, we assume here that the analytical formula may be extrapolated to somewhat higher mass. For this we used the analytical expressions derived by Cantó et al. (1995), which give good agreement with Popham's result for a wide range of parameters in the $1 M_{\odot}$ case. Figures A. $2+\mathrm{A} .3$ show the temperature distribution of our simple empirical BL compared with the distributions computed with the model of Popham et al. (1993). The difference between the temperature distributions for $\alpha=0.10$ and $\alpha=0.01$ in Figs. A. $2+$ A. 3 is consistent with the results of Godon (1996) (Sect. A.4).

\section{Appendix B: The input data}

\section{B.1. UV spectra from IUE}

The input data for the analysis consisted of the reprocessed low dispersion (ULDA and NEWSIPS taken from the archives at VILSPA, GSFC, and STSci) UV spectra of $40 \mathrm{PMS} \mathrm{Be} / \mathrm{Ae} / \mathrm{Fe}$ stars and 3 cTTs and the visual magnitudes of the stars at the moment of the exposures. The SW and LW of each star spectrum were joined at $1950 \AA$, and in cases where the large aperture (LAP) spectrum was overexposed, we used (parts of) the small aperture (SAP) spectra instead, if available (see Table F.6 for details). 
For stars visible from the ESO at La Silla, Chile $\left(\delta<+20^{\circ}\right)$, we collected photometric data on the Walraven (WULBV) system. If the $V^{\mathrm{J}}$-brightness derived from the Walraven photometry (Lub \& Pel 1977) agrees with $V_{\text {obs }}^{\text {iue }}$, we used the $W$-band flux (centered at $3236 \AA$, and indicated by (w) in the figures) to check the observed flux at the far end of the IUE LW-range. In cases where the LW images were overexposed this $W$-band flux became essential in the analysis of the spectra (e.g. Fig. 96).

The stars for which Walraven photometric observations have been obtained are indicated in Table 1 Col. 9 by $(+)$; see also de Winter et al. 2001. It should be noted that for variable stars we do not have Walraven photometry for all brightness phases (e.g. UX Ori, BF Ori). Also in some cases (CQ Tau, V350 Ori) the Walraven fluxes are too high to be indicated in the figure. This may perhaps be caused by the difference in diaphragm of the Walraven photometer and the IUE Field camera, which becomes important if the star has a nebulae around it that radiates in the UV.

Immediately before an IUE spectral observation, the Fine Error Sensor (FES) in the Field Camera Mode generally estimates a $B$-band flux of the object in terms of FES-counts. The derivation of $V^{\mathrm{J}}$ taken before 1991 was made with the FES calibration given by Imhoff \& Wasatonic (1986) and Barylak (1989), and for observations between 1-1-1991 and 1994, we used the calibration by Pérez (1991). We estimated the uncertainty of this procedure at $\sim 0$ m 05 . After 1994 the FES could not be used for accurate photometric measurements because of the occurrence of stray light in the Field Camera. For a number of observations we secured simultaneous ground-based photometry on the Johnson-, Strömgren-, or Walraven-systems that support the FES calibration method. The FES magnitudes, converted to $V^{\mathrm{J}}$, are listed in Tables 2-6 as $V_{\text {obs }}^{\text {iue }}$ for each set of spectra.

\section{B.2. Distances}

Most distances of stars associated with the nearby $(d<300 \mathrm{pc})$ star-formation regions (RSF) Taurus-Auriga, Ophiuchus, Lupus, Scorpius, Corona Australis, and Chamaeleonis and those of the isolated Herbig stars HD 163296, HD 104237, and HD 100546 have been evaluated by the Hipparcos project (ESA 1997). For each star the distance is given in Table 1 Col. 7 and Appendix C. The large uncertainties for some distances reflect the nebulous character of these objects. For some stars in our sample (HD 139614, HD 169142, and HD 142666) not observed by Hipparcos, we took the estimates of Sylvester et al. (1996). For the Herbig star candidates HD 95881 and HD 98922, we estimated a spectroscopic parallax. Distances $\gtrsim 350$ pc could not be determined with Hipparcos and were taken from other works (e.g. Warren \& Hesser were, for the Orion stars).

\section{B.3. Spectral type of the stellar photosphere}

Spectral classifications (Table 1) were obtained from two sources: photometric classifications from the position of the object in the $(B-V)$ vs. $(U-B)$ two-colour diagram (TCD hereafter) on the Johnson or Walraven photometric systems and spectroscopic classifications from high-resolution visual spectra. In Table 1 we give the results of both methods, where most of the spectroscopic classifications were taken from FJ84, HB88, and TWP94. Since the $W^{\mathrm{W}}, U^{\mathrm{W}}$, and $U^{\mathrm{J}}$ photometric passbands are not in the wavelength range of the visual spectroscopy, we may expect different results from the two classification methods.

\section{B.4. Foreground extinction}

All the spectra (i.e. of both standard stars and PMS stars) were de-reddened for foreground extinction before using them in the model-calculation. This was done with the analytical UVextinction law $A_{\lambda} / A_{\mathrm{v}}$ for $R^{\text {is }}=3.1$ from Cardelli et al. (1988, 1989). The $\boldsymbol{E}^{\text {is }}$-values of the PMS stars are listed in Table 1 Col. 4 and are usually based on the stars in their neighbourhood. However, in the cases of R CrA, HD 97048, and Z CMa, it was found that $R^{\text {is }}>3.1$ due to the presence of intercluster clouds, so we used these anomalous values for the interstellar de-reddening (Sects. C.42, C.30 for details).

\section{B.5. Disc inclination}

An indication of the value of the inclination angle $i$ of the disc, which is defined as the angle between the line-of-sight and the rotation axis of the disc, was obtained from several independent observational arguments. In principle, the inclination could be determined from the IR part of the SED where the contribution of the cool outer parts of the disc is largest. However, in this part contributions from other sources, e.g. circumstellar dust and dust in nearby reflection nebulosity may also be important. Fortunately the shape of the UV spectrum in the wavelength range of the IUE is not very sensitive to the value of $i$, so that only rough estimates of the inclination are needed. The various methods used to obtain estimates of the disc inclination are discussed in Appendix D. The results of these estimates are listed in Table 1 Col. 10 with index E for "edge-on" $\left(i \geq 60^{\circ}\right)$ and $\mathrm{P}$ for "pole-on" $\left(i \leq 20^{\circ}\right)$. The observational arguments for $\mathrm{E}$ or $\mathrm{P}$ are given in the columns X, Y, Z.

In column $X$ the type of variability in the TCD is listed $(\mathrm{r}=$ along the reddening line; $\mathrm{o}=$ other type of variability; $\mathrm{n}=$ no significant variability, $\dagger$ denotes stars that had variability along the reddening line in the past, i.e. changed from type $\mathrm{r}$ to $\mathrm{n})$. In column $\mathrm{Y}$ we give the $E W(\AA)$ of the $2400 \AA$ absorption-feature in the low-resolution UV spectra $\left(E W_{\mathrm{Fe}}\right.$ hereafter, Fig. D.1). Column Z shows the shape classification (as introduced by Reipurth et al. 1996) of the $\mathrm{H} \alpha$-emission profile of each star.

\section{Appendix C: Stellar data used for the analyses}

Our aim is to give here an updated review of the information that is relevant for the choice of the stellar input parameters of the analyses, such as distance, foreground extinction, and spectral type of the stars in Tables 1 and B.1. Section numbers correspond to the sequence numbers in the tables and the labels in Fig. 41.

\section{C.1. $X Y$ Per - HD 275877}

This object is a visual double star (ADS 2788, Table B.1). The ADS parameters have been confirmed by Hipparcos measurements and NIR imaging by Testi et al. (1998). The bright (western) component has a spectral type of early-A and the secondary component has type mid-A (HB88). The present photometric variability is not well known. According to Boyd (1936), the object is most of the time at maximum brightness with $V^{\mathrm{J}}=9.36$ and $(B-V)^{\mathrm{J}}=0.52$. The uncertain FES calibration at the date of observation gives $V^{\mathrm{J}}=9$. 0 . If we assume that the observation was made at $V^{\mathrm{J}}=9 \mathrm{~m} .36$ with $\Delta V^{\mathrm{J}}=0.6$ at that moment, we obtain $V^{\mathrm{J}}=9.85$ for the primary and $V^{\mathrm{J}}=10^{\mathrm{m}} 45$ for the secondary component. The distance of the object is uncertain. Adams et al. (1935) give a spectroscopic parallax of 0.'001, and 
Table B.1. Published double-star parameters and spectral types, see also Sect. 5.9 and Appendix C.

\begin{tabular}{lllccl}
\hline \hline $\begin{array}{l}\text { double } \\
\text { star }\end{array}$ & $\begin{array}{c}\text { spectral } \\
\text { types }\end{array}$ & $\begin{array}{l}\text { sep } \\
\left({ }^{\prime \prime}\right)\end{array}$ & $\begin{array}{c}\text { PA } \\
\left({ }^{\circ}\right)\end{array}$ & $\begin{array}{c}\Delta V^{J} \\
(\mathrm{mag})\end{array}$ & ref. \\
\hline XY Per & A2 II +A2-5 & 1.33 & 76. & $\sim 0.64$ & 7 \\
UX Ori & A2 II +?? & 0.022 & 257. & - & 5 \\
HD 150193 & A4 V +?? & 1.09 & 225. & $\sim 3.5$ & 7,12 \\
BF Ori & A5 +?? & - & - & - & 5 \\
HR 5999 & A5 III+IR & 1.44 & 112. & $\geq 3.65$ & $9,10,13$ \\
HD 144432 & A9 III+K0-5 & 1.4 & 353. & - & 16 \\
NX Pup & F2 III +G5 IV & 0.126 & 63. & 0.64 & $4,6,7$ \\
BD+46 ${ }^{\circ}$ 3471 & A2-4 +IR & 4.18 & 43. & $<0.14$ & 12 \\
HK Ori & A4 V +IR & 0.34 & 41. & $\sim 2.9$ & 9,10 \\
AK Sco (ap) & F5 V +F5 v & 0.001 & 185. & 0.42 & 2 \\
AK Sco (peri) & & & & 0.09 & \\
Z CMa & F5 Ib +B0 & 0.10 & 122.5 & 2.1 & 3 \\
HD 199143 & F6-8 +K7-M0 & 1.078 & 325. & - & 1,15 \\
V380 Ori & A2 V +IR & 0.134 & 204. & $\geq 2.6$ & 9,10 \\
KK Oph & A6 V +IR & 1.61 & 247. & $\sim 1.0$ & 10,12 \\
HD 98922 & A0 +?? & - & - & - & 8,14 \\
\hline
\end{tabular}

1: v.d.Ancker et al. (2000) 7: ESA (1997)

2: Andersen et al. (1989)

3: Barth et al. (1994) 8: de Groot (1971)

13: Stecklum et al. (1995)

4: Bernacca et al. (1993)

5: Bertout et al. (1999)

6: Brandner et al. (1995)

9: Leinert et al. (1994)

10: Leinert et al. (1997)

12: Pirzkal et al. (1997)
15: Jayawardhana \&

Brandeker (2001)

16: Rossiter (1955)

the Hipparcos Catalogue (ESA 1997) gives $d=120_{-36}^{+88} \mathrm{pc}$; but to obtain this value $17 \%$ of the measurements had to be rejected, so that this value is unreliable (AWT98). However, it is highly probable that XY Per is located in DC L1449 of RSF 1 Per B, which has $d \sim 350 \mathrm{pc}$ (Ungerechts \& Thaddeus 1987). For HD 275874 (A4) in the immediate surroundings (at 8'.4, with a comparable proper motion) we derive $\boldsymbol{E}^{\text {is }} \sim 0$. 19 .

\section{C.3. UX Ori - HD 293782}

The spectral classification in the UV (Tjin A Djie et al. 1984; Grady et al. 1995) agrees with that in the visual: A2-3 IIIe (HB88). Its photometric and polarimetric variability is similar to other UXORs (Zajtseva 1973; Zajtseva \& Kolotilov 1973; Voshchinnikov et al. 1988; Evans et al. 1989; Bibo \& Thé 1990). This peculiar variability has been interpreted by Grinin (1988) and by Voschinnikov (1989) in terms of eclipses of an inclined, oblate spheroidal dust envelope by optically-thick dust clouds passing the line-of-sight. The Hipparcos measurements give a lower limit for the distance of $130 \mathrm{pc}$ (AWT98). The star is located in DC L1616 near the Orion OB1C association. For UX Ori we adopted the parameters of this association: $d=400-430 \mathrm{pc}$, $\boldsymbol{E}^{\text {is }}=0$. m 06 (Warren \& Hesser 1978). Bertout et al. (1999) give indications that UX Ori is a double star (Table B.1).

\section{C.4. HD $163296-M W C 275$}

The visual spectrum has been classified as A0 ve by Thé et al. (1985a) and as A7 Ve by Fz85. Since the star is not associated with a star-formation region and does not show significant photometric variability, it has not been considered as a typical Herbig Ae star. In spite of the lack of brightness variations, its spectrum shows strong variability in the emission-lines of $\mathrm{H} \alpha$, Ca II K, and Mg II h,k on a time-scale of several hours (Thé et al. 1985b; Baade \& Stahl 1989b; Catala et al. 1989). The UV continuum shortward of $1600 \AA$ varies on the same time-scale.

This variation is probably not caused by the presence of a double-star companion, since radial velocity measurements by Baade \& Stahl (1989b) show no evidence of variation. Catala et al. (1989) explain the variability in terms of the rotational modulation of an outflow from active regions at the stellar surface. The distance of the star has been determined from Hipparcos measurements: $122_{-13}^{+17}$ pc (ATT97). Its foreground excess $\boldsymbol{E}^{\text {is }}$ is negligible. The inclination was derived from a redshifted Ly $\alpha$-component (Appendix D.4, Blondel et al. 1993): $57^{\circ}$ and from CO emission (Mannings \& Sargent 1997): 58 ${ }^{\circ}$. STIS imaging in the visual (Grady et al. 2000) confirmed the presence of the disc, which extends to at least $450 \mathrm{AU}$ from the star and has $i \sim 60^{\circ}$. STIS imaging near Ly $\alpha$ (Devine et al. 2000) confirmed the presence of in- and outflow jets with $v_{\text {rad }} \sim$ $350 \mathrm{~km} \mathrm{~s}^{-1}$.

\section{C.5. HD 150193 - MWC863 - V2307 Oph}

Its visual (FJ84) and UV spectra (this paper) are very similar to those of HD 163296. In the visual the star was classifed as A4e (Fz85). There are no indications of photometric variability, but spectral variations on short time-scales similar to HD 163296 have not been looked for. In spite of its location, the connection of HD 150193 to the Ophiuchus DC L1729 in Sco Rl at $d \sim 160$ pc was considered uncertain (Elias 1978a), but this connection has now been confirmed by Hipparcos measurements, $150_{-30}^{+50} \mathrm{pc}$ (ATT97), which also reveal that the star is a double system (Table B.1). Because this distance is close to the minimum distance of the Ophiuchus complex, we assume that the foreground excess is small e.g. 0.03 . Our high-resolution UV spectrum shows the presence of a red-shifted stellar component to the Ly $\alpha$-emission. From the profile, $i \sim 67^{\circ}$ was estimated (Appendix D.4).

\section{C.7. BF Ori $-B D-06^{\circ} 1259$}

The visual spectrum of BF Ori is usually classified as A5e+sh (HB88, Rostopchina 1999). Photometric and polarimetric observations (e.g. Kilkenny et al. 1985; Herbst et al. 1987; Hutchinson et al. 1994) show large and irregular variations, similar to other UXORs. The interpretation of these data can therefore be similar (Grinin et al. 1988) to the observational results of WW Vul and UX Ori (Sect. C.3). The Hipparcos measurements give a lower limit to the distance of $210 \mathrm{pc}$ (AWT98). Its association with M42 in RSF 1 Ori A suggests $d \sim 400-450 \mathrm{pc}$ and $\boldsymbol{E}^{\text {is }}=0.06$. Bertout et al. (1999) indicate that this is a double star (Table B.1).

\section{C.8. HD $31648-M W C 480$}

From its visual spectrum Merrill et al. (1942) and Burbidge \& Burbidge (1954) concluded that HD 31648 is a peculiar shell star (type A3ep) with variable shell components in the lines (Jaschek et al. 1988; Morrison 1997), similar to HD 163296 (Sect. C.4) and HD 150193 (Sect. C.5). Another similarity to these stars is that there are no indications of strong photometric variability of HD 31648 (Pogodin 1987). On the other hand, the three stars share several spectroscopic properties with the Herbig Ae stars: P-Cygni profiles of the strong emission-lines of the Balmer series and of $\mathrm{Mg}$ II h, $\mathrm{k}$, a strong Ly $\alpha$-emission and a NIR excess. Thus it seems jusified to consider these stars as PMS objects. Its location in the neighbourhood of $\mathrm{AB}$ Aur suggests that HD 31648 may belong to RSF 1 Tau. The Hipparcos measurements (AWT98) give $d=131_{-18}^{+24} \mathrm{pc}$ for HD 31648 and $141_{-17}^{+23} \mathrm{pc}$ 
for $\mathrm{AB}$ Aur, we assume for HD 31648 that $\boldsymbol{E}^{\text {is }}=0$ m.02, equal to that of AB Aur. From the CO interferometry of HD 31648 , Dutrey (2000) derived $i=38^{\circ}$.

\section{C.9. HR 5999 - V856 Sco - HD 144668}

The visual spectral classification of this star in the Lupus T3 association is A5-7 IIIe (Bessell \& Eggen 1972; Tjin A Djie et al. 1989). The object is strongly and irregularly variable in brightness (Bessell \& Eggen 1972; Thé \& Tjin A Djie 1978; Thé et al. 1981; Baade \& Stahl 1989a), as well as in the line profiles and line strengths. Although the photometric behaviour of HR 5999 is similar to that of the $U X O R \mathrm{~s}$, it is commonly not considered to be a member of this group, because the star does not show the "blueing branch" in the CMD of the UXORs. An explanation for the absence of this branch in the CMD of HR 5999 may be the depletion of small dust grains in its halo. The distance of HR 5999 was derived by Bessell \& Eggen (1972) and by Thé $\&$ Tjin A Djie (1978) on the assumption that it is the same as that of the nearby $\left(45^{\prime}\right)$ common radial and proper motion companion HR 6000 (HD 144667). Its photometric classification is B6 V, which implies that $d=270 \mathrm{pc}$ and $\boldsymbol{E}^{\text {is }}=0$. 06 . However, HR 6000 is a He-weak peculiar star, so the classification from its spectrum should give a slightly cooler type (Garrison 1978; Eggen 1998), about B7-8 V, which corresponds to $d=234 \mathrm{pc}$ and $\boldsymbol{E}^{\text {is }}=0.03$. This value is an upper limit for $\boldsymbol{E}^{\text {is }}$ in the direction of HR 5999/6000. Since there are no i.s. Na I D absorption lines in the spectrum of HR 6000 , its $\boldsymbol{E}^{\text {is }}$ is probably negligible. We shall assume that both stars have the same foreground extinction. The Hipparcos measurements have resulted in a value of $240_{-39}^{+61} \mathrm{pc}$ for HR 6000 and $210_{-30}^{+50} \mathrm{pc}$ for HR 5999 (ESA 1997, AWT98). These values are still in the range of recent estimates of $140-250 \mathrm{pc}$ for the distance to the Lupus T3 cloud (Hughes et al. 1993), with which both stars are thought to be associated. The high-resolution Ly $\alpha$-profile of HR 5999 shows a redshifted component, and $i=53^{\circ}$ could be derived from its profile (Blondel et al. 1993; Appendix D.4). The star was detected to be a PMS $\delta$ Scuti variable (Kurtz \& Marang 1995) and has a double-star companion (Table B.1).

\section{C.10. HD $39060-\beta$ Pic}

This star is generally considered as the prototype of a young A5-7 IV-V star in the phase of its arrival on the ZAMS. Its evolutionary status is discussed by Paresce (1991), Lanz et al. (1995), and Brunini \& Benvenuto (1996). The Hipparcos measurements give $d=19.3_{-0.2}^{+0.2}$ pc (Crifo et al. 1997). Its $\boldsymbol{E}^{\text {is }}$ is negligible (Sylvester et al. 1994). The star has a thin circumstellar dust disc with $i \sim 90^{\circ}$ (Smith \& Terrile 1984). For our UV3C analysis we assume that the (hypothetical) accretion disc remnant has the same inclination. The star was recently discovered to be a ZAMS $\delta$ Scuti variable (Koen 2003).

\section{C.11. HD $104237-D X$ Cha}

This star in ChaIII was classified by WW88 as A0e and has been identified as a Herbig Ae star by $\mathrm{Hu}$ et al. (1989). Along with $V^{\mathrm{J}}=6^{\mathrm{m}} \cdot 6$ it is the brightest Herbig Ae star so far. From its low-resolution UV spectrum, Hu et al. (1991) classified HD 104237 as A4 Ve. Monitoring photometry and spectroscopy during 5 successive nights in 1991, repeated in 1992, showed short-term variability in the emission-line fluxes but no photometric variability: neither on short time-scales (hours or nights) nor between the two years (de Winter et al. 2001). Highresolution UV spectra, taken with IUE, show P-Cygni profiles for the $\mathrm{Mg} \mathrm{II} \mathrm{h}, \mathrm{k}$ emission-lines and strong indications of such profiles for the emission-lines of C II, Si IV, and C IV. Improved resolution and $\mathrm{S} / \mathrm{N}$ for these profiles, obtained with the GHRS of HST, suggests the presence of a bi-conical disc wind, originating in the inner part of the accretion disc (Brown et al. 1997). The distance of HD 104237 was estimated (Hu et al. 1991) from its close $\left(2^{\prime}\right)$ common-proper motion companion $\varepsilon$ Cha (HR 4583, B9 v), which has $d \sim 88 \mathrm{pc}$. However, by assuming that $\varepsilon$ Cha is a ZAMS star and by accounting for the double-star nature of $\varepsilon$ Cha itself, Eggen (1998) improved the estimate to a value of $\sim 111$ pc. This is just within the range of the Hipparcos (ATT97) measurement: $116_{-7}^{+8}$ pc. HD 104237 does not show much photometric variability: $V^{\mathrm{J}} \sim 6.60$. The UV spectrum indicates a type $\mathrm{A} 7 \mathrm{IV}$, which gives $E(B-V)^{\mathrm{J}}=0.02-0.04^{\mathrm{m}}$. We assume that $\boldsymbol{E}^{\text {is }}$ corresponds to the lowest value; i.e. $\boldsymbol{E}^{\text {is }}=0.02$. Knee \& Prusti (1996) found evidence that these stars are located in a hole of the molecular cloud in which they were formed, so that they have almost no foreground extinction. From the red-shifted component of $\operatorname{Ly} \alpha$-emission, we derived $i=53^{\circ}$ (Blondel et al. 1993, Appendix D.4). The $v \sin i$-value from Table 1 was estimated by us from lines in the visual spectrum of HD 104237 (de Winter \& van den Ancker 1991). Also this star was found to be a PMS $\delta$ Scuti variable (Donati et al. 1997; Kurtz \& Müller 1999).

\section{C.12. HD $169142-M W C 925$}

The star was identified as a nearby B9 V star by WW88. Since it has $\mathrm{H} \alpha$-emission and a strong NIR excess (Oud92) it was recognised as a Herbig star (possibly a double star). The photometric variability of HD 169142 is unknown. From its visual spectrum Dunkin et al. (1997a,b) classified the star as A5 Ve. For this type Sylvester et al. (1996) derived $d=145 \mathrm{pc}$ and $\boldsymbol{E}^{\text {is }}<0$. 14 . For A7 ve we find with Sylvester's $(B-V)^{\mathrm{J}}=0.29$ that $d=151 \mathrm{pc}$ and $\boldsymbol{E}^{\text {is }}=0.0$.

\section{C.13. HD $36112-M W C 758$}

Oud92 classified this star as A3e and MBW98 identified it as an Herbig Ae star. Variations in the $\mathrm{H} \alpha$-emission profiles on a time-scale of days were observed by Beskrovnaya et al. (1996). No photometric variations are known. From the Hipparcos measurements $d=200_{-40}^{+60} \mathrm{pc}$ was derived (AWT98), and from the UV spectrum we conclude that the spectral type must be A7 III. With $(B-V)^{\mathrm{J}}=0^{\mathrm{m}} 29$ (de Winter et al. 2001) we then find that $\boldsymbol{E}^{\text {is }}<0.07$.

\section{C.14. $H D 139614-C D-42^{\circ} 10650$}

WW88 classified this star close to RSF Sco/Oph as A7 Vz and MWM98 recognised it as a Herbig Ae star. Profiles of $\mathrm{H} \alpha(\mathrm{em})$, Na I D and He I (em) was observed by Dunkin et al. (1997a) and MWM98. The star may be a double star. Its photometric variability is still unknown. From Johnson photometry Sylvester et al. (1996) derived $d=157 \mathrm{pc}$ and $\boldsymbol{E}^{\text {is }}<0$. 03 .

\section{C.15. HD $142666-V 1026$ Sco}

Also this object is situated near RSF Sco/Oph. The object was classified as an A3 Vz star by WW88 and identified as a Herbig Ae star by MWM98. Profiles of $\mathrm{H} \alpha(\mathrm{em}), \mathrm{He} \mathrm{I}$, and 
Na I D were obtained by Dunkin et al. (1997a) and MWM98. HD 142666 is photometrically variable (MBW98), which was confirmed by the observations with the Tycho instrument aboard the Hipparcos satellite (Makarov et al. 1994). From Johnson photometry of the star Sylvester et al. (1996) derived a spectral type A8 ve, $d=114 \mathrm{pc}$ and $\boldsymbol{E}^{\text {is }}<0$. 30 . From the $E W^{\prime}$ 's of the Na I D lines, observed by Dunkin et al. (1997a) we derive with the Strömgren (1948) doublet method and the relation of Hobbs (1974) that $\boldsymbol{E}^{\text {is }}<0$. 26 . MWM98 obtained $\boldsymbol{E}^{\text {is }}=0.22$ from Geneva photometry. The star is a PMS $\delta$ Scuti variable (Kurtz \& Müller 2001).

\section{C.16. V346 Ori - HD 287841}

The star is a rapid, strongly $\left(\Delta V^{\mathrm{J}}=1 \mathrm{~m} .9\right)$ irregular-variable, and from the TCD we estimate a spectral type of A3-4. Its CMD shows a "blueing branch", which is typical for the UXORs. The photometric time-variability was studied by Koval'chuk (1985), who noted that deep $\left(\Delta m_{v} \sim 1-2^{\mathrm{m}}\right)$ fadings in brightness occur abruptly within 1 day and are followed by a return to the initial brightness in 1-5 days. The amplitude of the fading increases towards the UV. High resolution spectra (de Winter 1991) show $\mathrm{H} \alpha$-emission filling-in the photospheric absorption profile. Other low-resolution spectra show many metallic absorption lines, including the Ca II (2) red-triplet, which indicate a late-A spectral type that is in agreement with the classification A5 III of Zajtseva (1986). The lower limit for the distance, obtained by Hipparcos (ATT98) is $130 \mathrm{pc}$ so that it is not certain whether this star is a member of the Orion OBla complex. V346 Ori was recently detected to be a PMS $\delta S c u t i$ variable (Pinheiro et al. 2003).

\section{C.18. V351 Ori - HD 38238}

The photometric behaviour of this star, located in $M 78$, a cluster in DC Lyn 1630, is peculiar: before $1984 / 5$ it behaved like a Herbig Ae star with strong variations $\left(\Delta V^{\mathrm{J}}=3\right.$ m. 3$)$ with a "blueing branch" in the CMD, while after 1985 the star is at maximum brightness and rarely varies. In this respect its photometric behaviour is similar to that of BN Ori (Sect. C.19) and FU Ori (Sect. C.23). A flare was reported in 1982 by Koval'chuk (1984, 1986). Spectroscopic information from the period before 1985 is scarce, but from a low resolution blue spectrum Strom et al. (1975) estimated a spectral type of A0-2 from the strength of the $\mathrm{Ba}$ II and Ca II absorption lines. In a lowresolution IDS spectrum taken in 1985 by H. Cuypers (unpubl.), the $\mathrm{H} \alpha$ line is partly filled-in. The high-resolution spectra by van den Ancker et al. (1996) confirm this filling-in and indicate a spectral type A5-7 IIIe. This spectral type is in agreement with the photometric classifications of Kilkenny et al. (1985) and Zajtseva (1986). Hipparcos observations give a lower limit for the distance of $210 \mathrm{pc}$ (AWT98). Because of its location in the association Ori OB $1 \mathrm{bl}$ we assume $d=450 \mathrm{pc}$ and $\boldsymbol{E}^{\text {is }}=0$. 06 for V351 Ori. Marconi et al. (2000) observed that this star is a PMS $\delta$ Scuti variable.

\section{C.19. BN Ori - HD 245465}

This star near $\lambda$ Ori has a remarkable photometric history: between 1897 and 1947 the star was strongly and irregularly variable, with amplitudes up to $4 \mathrm{~m}$.(Dragomiretskaya 1965), similar to BF Ori (Sect. C.7), T Ori, and UX Ori (Sect. C.3). After 1947 the brightness increased by 0.5 with small variations and finally settled at $V^{\mathrm{J}}=9 \mathrm{~m} .65$, where it stayed since 1965 . From the TCD a spectral type of A5-F2 can be estimated, while spectroscopic classifications range from A7-F0 (Cannon 1931, Hb54) or A7-late-G (Shevchenko 1989). The later spectral types may be interpreted by the presence of outer layers in the envelope of the star, which contribute to a certain stratification in this envelope, similar to that present in the envelope of FUORs in their post-outburts phase. The similarity of the lightcurve and spectroscopic features between BN Ori and the "classical" FUORs suggest that BN Ori is related to this class (Shevchenko et al. 1997). In this scenario the photospheric classification corresponds to A7-F0 III. We will assume that the star belongs to the $\lambda$ Orionis association $(\mathrm{Cr} 70)$ close to $R S F 1$ Ori $B$ and therefore adopt $d=400 \mathrm{pc}$ (from the mean parallax determined by Artiyukhina 1960) and $\boldsymbol{E}^{\text {is }}=0$ m 06 (from the Na I D lines determined by Shevchenko et al. 1997).

\section{C.20. $H D 35929-B D-08^{\circ} 1128$}

The spectral classification in the HD catalogue is A5. From his photometry on the Strömgren system Olson (1979) derived spectral type F5 and from their $U B V R I$-photometry Miroshnichenko et al. (1997) classified the star as A8 Vz, which implies $d \sim$ $100 \mathrm{pc}$. However, the Hipparcos measurements led to $d \geq 360 \mathrm{pc}$ (AWT98). If we assume that the star belongs to the association Ori OBlc, with $d \sim 430 \mathrm{pc}$ and $\boldsymbol{E}^{\text {is }} \sim 0$. 06 (Warren \& Hesser 1978 ) it should be a giant. From the flux rise in the IUE SWspectral range we classify HD 35929 as F0 IIIe (with $M_{\mathrm{v}}=-0.3$ and $d=400 \mathrm{pc}$ ). This star is a PMS $\delta$ Scuti variable (Marconi et al. 2000).

\section{C.21. HD $144432-C D-27^{\circ} 10778$}

Visual spectra show a variable double $\mathrm{H} \alpha$-emission profile, a weak and broad He I 5876 ̊ in emission, broad Na I D lines with emission-components, the Ca II (2) red-triplet in emission, and an inverse P-Cygni profile for He I $(1.08 \mu \mathrm{m})$ (Pérez et al. 2004; Grady et al. 1996; Dunkin et al. 1997a,b, MWM98). There is no record of photometrical variability. This star in RSF Sco Rl was classified by WW88 as a ZAMS star of spectral type A9-F0 at $d \sim 120$ pc. Sylvester et al. (1996) arrives at the same conclusion from the Johnson photometry. If the star is a member of Sco OB2-2 (Pérez et al. 2004) then $d=145$ pc. However, the Hipparcos observation gives $d \geq 200 \mathrm{pc}$ (AWT98). An $\boldsymbol{E}^{\text {is }}<0$. 03 was estimated by Sylvester et al. (1996). MWM98 obtain $\boldsymbol{E}^{\text {is }} \leq 0^{\mathrm{m}}$. 15 , but the application of the Strömgren Doublet method to the Na ID EW's observed by Dunkin et al. (1997a) gives us $\boldsymbol{E}^{\text {is }} \leq 0$. 02 . The low value of the foreground extinction is common to the other stars in the neighbourhood of $R S F$ Sco/Oph: HD 139614 (Sect. C.14) and HR 5999 (Sect. C.9). HD 144432 is a visual double star (Table B.1).

\section{C.22. $B D+47^{\circ} 4206$}

From objective prism spectra the star was classified as A9 III. At present it is not known if $\mathrm{H} \alpha$ has an emission component. There is no record of photometric variability. The star is a member (n0.19) of the young BM And association with $d=440( \pm 100) \mathrm{pc}$ and $\boldsymbol{E}^{\text {is }}=0.05$ (Aveni \& Hunter 1969), which is in the range of the Hipparcos result: $260_{-74}^{+166}$ pc (ESA 1997). 


\section{C.23. FU Ori $-B D+09^{\circ} 5427$}

This peculiar star is the result of an outburst of a 16th magnitude star in the last months of 1936. Since the outburst the brightness has decreased slowly by a small amount until it reached its present value of $V^{\mathrm{J}}=9 \mathrm{~m}$. 3 . Its visual spectrum after the outburst has been described by Hb66, who classified the photospheric component as F2:p I-II from the Balmer lines and the lowexcitation lines of neutral and singly ionised metals. The envelope is of type K-M deduced from the Ca II (2) red-triplet, which points to a stratification of the atmosphere (see also BN Ori; Sect. C.19). Similar to Z CMa (Sect. C.30) the low-resolution UV spectrum of FU Ori has been analysed by Kenyon et al. (1989), but in these analyses the IUE SW-part (in which the BL dominates) has not been taken into account. Since the precursor of FU Ori was a member of the Ori T4 association (Kh59) it was presumably a T Tauri star, so that its mass cannot be much larger than $\sim 1 M_{\odot}$. Because of its location in the RSF Orion, we adopt $d=450-500 \mathrm{pc}$ and $\boldsymbol{E}^{\text {is }}=0.06$.

\section{C.25. $C Q$ Tau - HD 36910}

CQ Tau is situated in the T-association Tau T4 (Kh59). This star is strongly and irregularly variable, similar to other UXORs. From their collection of blue objective prism spectra obtained at various brightness phases, Götz \& Wenzel (1968) concluded that at bright phases $\left(V^{\mathrm{J}}<10^{\mathrm{m}} 4\right)$ the star had spectral type A-F0, wheras during the fainter phases it could be classified as A2-8. In addition, for phases fainter than $V^{\mathrm{J}}=11^{\mathrm{m}} 1$ the spectral type of CQ Tau could vary between A2 and F7. These classifications were mainly based on the strength of the Balmer and CaII K lines and of the strength of the G-band. From model-fitting of the wings of the $\mathrm{H} \alpha$-line in a spectrum taken at $V^{\mathrm{J}}=10 \mathrm{~m} 46$, Berdyugin et al. (1990) concluded to a type F0 III. The TCD photometric classification gave A8. Artyukhina (1959) derives $d=137$ pc from the kinematic parallax. Hipparcos gives $d=100_{-17}^{+25}$ pc (AWT98). Götz \& Wenzel (1968) derived $d=220 \mathrm{pc}$ for the nearby (2'.) G0 V reference star $f$ with an $\boldsymbol{E}^{\text {is }}=0$ m. 10 . This G-star has not been observed by Hipparcos. If we assume a uniform colour excess over the distance of $220 \mathrm{pc}$ we then estimate that $\boldsymbol{E}^{\text {is }}=0$ m 068 for CQ Tau.

\section{C.26. NX Pup $-C D-44^{\circ} 3318$}

This variable star in the head of the cometary globule $C G 1$ of the Gum nebula, can be classified as F4-5 and sometimes F0-2 from its position in the TCD (photometry by Brand et al. 1983; Tjin A Djie et al. 1984; Ng 1986). The short time-scale variability is correlated with a variable UVE for different brightness stages of the star. From its visual spectrum the star has been classified as F0-2 IIIJ (Irvine 1975; Brand et al. 1983; Reipurth 1983), in particular because of the presence of the G-band. On the other hand, several features in the visual spectrum resemble those of the Herbig B9e star HD 250550 (Reipurth 1983) and the SW-part of the UV spectrum is very similar to that of an A0 Ie type star (Tjin A Djie et al. 1984), which is consistent with the flux distribution in the UV as observed by ANS (de Boer 1977). In agreement with this the SED from UV to NIR could be matched by the flux distribution of a strongly reddened A0 III star with an anomalous circumstellar extinction law (Tjin A Djie et al. 1984).

The presence of the He I $5876 \AA$ line could support this classification (Böhm \& Catala 1995). However this line also occurs in late-type stars (e.g. RR Tau) and is highly variable in strength and can even disappear (Grinin et al. 2001), so that classification based on the occurance of the $5876 \AA$ line is uncertain. Recently, high angular resolution observations with the Fine Guidance Sensor \#3 aboard HST show that NX Pup is a double star with a separation of $0 .^{\prime} 126\left( \pm 0{ }^{\prime} 007\right)$, a PA of $63^{\circ}$, and a $\Delta V^{\mathrm{J}}$ of 0.64 (Bernacca et al. 1993, Table B.1). These parameters were confirmed by Hipparcos measurements. The companion was detected in the NIR by Brandner et al. (1995, 1996), Tessier et al. (1994) and Schöller et al. (1996). This secondary probably has a spectral type between F2 II and G5 IV. From the association with the Gum nebula, we estimate $d=450-500 \mathrm{pc}$ (Brand et al. 1983). According to Krautter (1980) the $\boldsymbol{E}^{\text {is }}$ in the direction of NX Pup is negligible.

\section{C.27. $B D+46^{\circ} 3471-V 1578 C y g$}

This star was shown to be a double star (Pirzkal et al. 1997, Table B.1). The few available photometric observations (taken years apart) show differences, but the course and the time-scale of the variability are not known so far. The position of the star in the TCD corresponds with spectral type A5. Classifications from the visual spectrum of $\mathrm{BD}+46^{\circ} 3471$ range from A2e (Str72) to A4e (Fz85, Catala et al. 1986). On the other hand, the spectrum of this star in the blue and UV is very similar to that of NX Pup (Sect. C.26), which suggests that $\mathrm{BD}+46^{\circ} 3471$ has a spectral type F0-2 III. However, we have an additional argument to constrain the spectral type. From the high-resolution Na I D observations of Catala et al. (1986) we derive with the Strömgren doublet method (1948) a column density of $2.06 \times 10^{13} \mathrm{~cm}^{-2}$ for Na I. Since Na I can only be present in the foreground of the star, we obtain an $\boldsymbol{E}^{\text {is }}=0.31$ from the empirical relation between $\boldsymbol{E}^{\text {is }}$ and the i.s. H or Na I D column density of Hobbs (1974). Then the observed $(B-V)^{\mathrm{J}}=0{ }^{\mathrm{m}} 41$ and the values of $(B-V)_{0}^{\mathrm{J}}$ given by SK82 only permit spectral types earlier than A5. Our arguments do not constrain the distance. The Hipparcos measurements give only a lower limit of $d=280 \mathrm{pc}$ (AWT98). The star is probably a member of the young open cluster IC 5146, for which $d \sim 900( \pm 100)$ pc (Elias 78c). The value of $\boldsymbol{E}^{\text {is }}$ is in the same range as found for CMaRl (Shevchenko et al. 1999), which has $d \sim 1 \mathrm{kpc}$. The Na I D profiles in both cases indicate that part of the $\boldsymbol{E}^{\text {is }}$-values may be due to extinction in the association.

\section{C.28. HK Ori - MWC497}

The star is a double star system (Leinert et al. 1997, Table B.1) in which the secondary companion is much redder than the primary star and probably contributes very little to the UV fluxes of the system. The visual spectral classification of HK Ori is ambiguous and depends on the features which are given most weight. On the basis of the strength of the Balmer lines and the presence of faint He I and Ca I lines Joy (1949) and Hb60a suggested a type A4 V, but from the occurrence of He I and Fe II emission-lines Str72 concluded to B7-8. It is possible that the emission from the surrounding nebula contributes to the spectrum. Because of such ambiguities later catalogues are less explicit and only quote A-Fe (Fz85) or Ae (HB88). HK Ori is an irregular variable with a mean position in the TCD of the Johnson and Walraven photometric systems which corresponds with F5. If the star is a member of the Ori T1 association with $d=400-460 \mathrm{pc}(\mathrm{Kh} 59)$ and $\boldsymbol{E}^{\text {is }}=0$. 06 and if we assume that its c.s. excess is small, its visual magnitude $V_{\mathrm{obs}}^{\text {iue }}=11.5$ constrains $M_{\mathrm{v}}$ to $3.3-2.8^{\mathrm{m}}$, which corresponds to type F0-2 V or F8 IV to early-K IV (SK82). 
P. F. C. Blondel and H. R. E. Tjin A Djie: Modeling of PMS Ae/Fe stars using UV spectra, Online Material p 23

\section{C.29. AK Sco - HD 152404}

AK Sco is situated in the border of the upper Sco subgroup of the Sco-Cen association. Considering its spectrum and in particular the presence of strong Li I absorption, Hb60b classified AK Sco as a F5 IVe PMS star. Later Andersen et al. (1989) showed that the star is a double-line spectroscopic double star (Table B.1). Accurate radial velocity measurements resulted to the orbital elements: $P_{\text {orb }}=13.6, \mathrm{PA}=185^{\circ}, e=0.47$ and a massratio of $\sim 1$. From the orbital elements, the spectral type, the intrinsic colour indices, and the rotation velocities, Andersen et al. (1989) modeled the system by comparing it with the MS double star RZ Cha. The AK Sco-system probably consists of two $1.5 M_{\odot}$ F5 IV stars with an orbital inclination of $63^{\circ}$ and semimajor axis of $35 R_{\odot}$. The distance and colour excess (at maximum brightness), derived by Andersen et al. (1989) for spectral type F6 Ve are $200( \pm 40) \mathrm{pc}$ and $0 \mathrm{~m}^{\mathrm{m}} 13( \pm 0.02)$. The Hipparcos measurements give $150_{-30}^{+40} \mathrm{pc}$ (AWT98). At this distance the separation of the components is too small to resolve them with the HST FES (Bernacca et al. 1995).

\section{C.30. Z CMa - HD 53179}

The star is a member of the CMaRl association with $d \sim$ 1150 pc (Claria 1974; Herbst et al. 1982). The visual spectrum of $\mathrm{Z} \mathrm{CMa}$ is complicated and shows simultaneous lines which are characteristic for early (B5-8) and late (mid-F) spectral types (Str72, FJ84, Covino et al. 1984; Hessman et al. 1991; Hamann $\&$ Persson 1992). The spectral varability and the lightcurve suggest that the star is related to the class of the FUORs. Hartmann et al. (1989) showed that the line profiles in the red and NIR part of the spectrum can be formed in a rotating accretion disc. From these data and the SED of Z CMa they derive $M_{\star} \sim 1-3 M_{\odot}$, an inner disc radius of $9-16 R_{\odot}$ and $\dot{M} \sim 10^{-3} M_{\odot} \mathrm{yr}^{-1}$.

These values had to be revised after the speckle interferometric detection of a companion star at 0.11 in the NIR (Koresko et al. 1991, Haas et al. 1993) and in the visual (Barth et al. 1994, Thiébaut et al. 1995, Table B.1). This companion of $2100 L_{\odot}$ dominates the IR part of the SED, wheras the FUORtype star of only $640 L_{\odot}$ dominates in the visual. Koresko et al. (1991) derived $M_{\star}=2.3 M_{\odot}$, an inner disc radius of $20 R_{\odot}$ and $\dot{M}=6 \times 10^{-4} M_{\odot} \mathrm{yr}^{-1}$ for the visual companion. Occasionally (Feb. 1987 and Jan. 2000) the IR primary dominates the visual spectrum with a spectral type B0 IIIe (van den Ancker et al. 1904) Optical spectropolarimetry by Whitney et al. (1993) confirms this picture and for the visual spectrum of the system the authors assume an effective spectral type F5 I, which leads to $(B-V)^{\mathrm{J}}=0.7$. They separated this into $\boldsymbol{E}^{\mathrm{is}}=0.2$ and $E(B-V)_{\text {ass }}^{\mathrm{J}}=0.5$ with $R=4.2$ for the dust in the CMaRl association. From a study of this association (Shevchenko et al. 1999) we conclude that $\boldsymbol{E}^{\text {is }}=0^{\mathrm{m}} 16$ and $E(B-V)_{\text {ass }}^{\mathrm{J}}=0^{\mathrm{m}} .20$. Similar to FU Ori (Sect. C.23) the UV spectrum of Z CMa has been analysed by Kenyon et al. (1989), but also in this analysis the IUE SW-part has been ignored. For Z CMa we use $d=1050( \pm 150)$ pc, which was derived for the CMaRl association (Shevchenko et al. 1999).

\section{C.31. $H D 199143-B D-17^{\circ} 6127$}

This star has an MK classification of F8 V, which was confirmed by van den Ancker et al. (2000) from its SED. These authors also reported many strong variable emission-lines in the spectra taken with IUE and EUVE and a rapid rotation $\left(v \sin i \sim 125 \mathrm{~km} \mathrm{~s}^{-1}\right)$ from lines in the visual. They suggested that the emission and
Table C.1. Data for HD 199143 from Neuhäuser et al. (2002).

\begin{tabular}{lllll}
\hline \hline & ST & $L_{\star}\left(L_{\odot}\right)$ & $M_{\star}\left(M_{\odot}\right)$ & $t_{\star}(\mathrm{Myr})$ \\
\hline A & F8 V & $2.4 \pm 0.25$ & $\sim 1.25$ & $\sim 20$ \\
B & M1 V & $0.06-0.1$ & $0.4-0.6$ & $\sim 20$ \\
\hline
\end{tabular}

the rapid rotation are due to an unseen nearby $\mathrm{T}$ Tauri companion, which is spinning up the F8 V star by episodic mass transfer. Such a companion was recently detected by high-resolution NIR imaging by Jayawardhana \& Brandeker (1991) and by Neuhäuser et al. (2002) at a distance of 1.' from HD 199143. They estimated the values given in Table C. 1 from the Hipparcos distance measurement $(47.7( \pm 2.4) \mathrm{pc})$ and the theoretical evolutionary tracks (Palla \& Stahler 1999). Almost simultaneous observations by Chauvin et al. (2002) essentially confirm these results, but their improved flux measurement in the J-band and the corresponding choice of a slightly later spectral type (M2 V) for the secondary star removes the excess in this band and therefore the need for circumstellar dust around this star. This does not give evidence of (recent) mass transfer from the secondary star. For our UV spectral analysis we assume that $\boldsymbol{E}^{\text {is }}<0$. 01 , since this value was needed in order to fit the Kurucz model with $T_{\text {eff }}=6200 \mathrm{~K}$ and $\log g=4.3$ (van den Ancker et al. 2000) to the observed SED of HD 199143A.

\section{C.32. V380 Ori $-B D-06^{\circ} 1253$}

The star is a double-star system (Leinert et al. 1994), in which the secondary companion is much redder than the primary star (the flux ratio is 0.31 at $2.2 \mu \mathrm{m}$ ) and probably contributes very little to the UV fluxes of the system. The doubler-star parameters are given in Table B.1. The classification of its visual spectrum gives B8-A2e (Hb60a), which is mainly based on the strength of the Balmer lines. On the other hand Herbig also noted some similarities with the spectra of the later type T Tauri stars and of Z CMa (Sect. C.30), in particular the strong emissioncomponents in many Fe II, Cr II, and Ti II lines. Later it has been argued that these lines may be formed in an envelope or in the surface wind around the star (Shevchenko 1994, 1998; Rossi et al. 1999). The Li I absorption line at $6707 \AA$ (Corcoran \& Ray 1995) may be due to the secondary component, which therefore should have a spectral type of F5 or later. The position of the star in the TCD of the Johnson and Walraven photometric systems varies irregularly in time between B4-B9 and F. These variations are similar to what is observed for NX Pup (Sect. C.26), and are clearly correlated with a variable UVE. The minimum value of $(U-B)^{\mathrm{J}}$ is zero, which corresponds with a type F. Since V380 Ori is located in the Ori T2 association (NGC 1999) of Ori OB1c, we assume that it has $d \sim 430 \mathrm{pc}$ and $\boldsymbol{E}^{\text {is }}=0$. 06 (Warren \& Hesser 1978). In this case the average brightness $V^{\mathrm{J}}=10^{\mathrm{m}} .5$ of the star leads to $M_{\mathrm{v}}=2 \mathrm{~m}$. 1 , that corresponds to either a type A4-6 V or F-G IV.

\section{C.33. NV Ori $-B D-05^{\circ} 1324$}

Spectroscopic classifications of this star range from F0 IV (Strand 1958) to F4-F8 III-V (Johnson 1964, HB88). Low resolution spectra CCD spectra obtained by van den Ancker (1996) in the visual and red show the presence of a weak G-band, a strong O I $7774 \AA$ absorption and the Ca II (2) red-triplet in absorption, probably filled in by emission, as is present in the Balmer lines. Johnson (1964) reports the presence of a weak Li I line. The position of the star in the Johnson TCD is F0-F2. The 
Table C.2. Derived distances (pc) for KK Oph depending on the spectral classification and the $\boldsymbol{E}^{\text {is }}$-value (in brackets).

\begin{tabular}{|c|c|c|c|c|c|c|c|c|}
\hline \multicolumn{3}{|c|}{ A5 (0.32) } & \multicolumn{3}{|c|}{$\overline{\bar{F} 0(0.17)}$} & \multicolumn{3}{|c|}{$\overline{\mathrm{F} 2(\sim 0 . \mathrm{m} 0)}$} \\
\hline $\begin{array}{l}\text { IV } \\
250\end{array}$ & $\begin{array}{l}\mathrm{V} \\
196\end{array}$ & $\begin{array}{l}\text { III } \\
\geq 350\end{array}$ & $\begin{array}{c}\mathrm{V} \\
172\end{array}$ & $\begin{array}{l}\text { IV } \\
226\end{array}$ & $\begin{array}{l}\text { III } \\
\geq 300\end{array}$ & $\begin{array}{c}\mathrm{V} \\
150\end{array}$ & $\begin{array}{l}\text { IV } \\
240\end{array}$ & $\begin{array}{l}\text { III } \\
\geq 360\end{array}$ \\
\hline
\end{tabular}

photometric variability is irregular, with a maximum amplitude of 0.6 . The star is located in the Ori T2 association of Ori OB1c, so that we also here assume that $d \sim 430 \mathrm{pc}$ and $\boldsymbol{E}^{\text {is }}=0.06$. From $V_{\text {obs }}^{\text {iue }}=10$. 0 we then find $M_{\mathrm{v}}=1 \mathrm{~m} .79$, corresponding with a spectral type F2-F4 III, that is in agreement with the spectroscopic and TCD classifications.

\section{C.35. KK Oph - AS 220}

The star is situated close to the edge of DC Barnard 59, which is probably part of the $\zeta O p h$ nebula. The visual spectrum of KK Oph was studied by FJ84 and by de Winter \& Thé (1990). It shows emission in $\mathrm{H} \alpha, \mathrm{H} \beta$ and [O I] at $6300 \AA$. Because of the strength of $\mathrm{H} \delta$ and $\mathrm{H} 8$ and the absence of He I the star was classified (MK) as A5-7e. The position of the star in the TCD of the Johnson and Walraven photometric systems is A8 to F4. In a new set of 478 photometric data between JD 2445881 and JD 2450672 (Herbst \& Shevchenko 1999) the brightness of the star varies between 9.40 and 12.8 with $(B-V)^{\mathrm{J}}=0.47$. If we assume that at $V_{\max }^{\mathrm{J}}=9.4$ the extinction was only due to interstellar foreground, we find the data given in Table C.2. For a type A5 IV we derive the same distance as determined by Wallenquist (1937) for the front side of the dark nebulosity ${ }^{9}$. If KK Oph is in front of the dark nebula, then $d \leq 250 \mathrm{pc}$, so that its spectral classification has to be restricted to A5-F6 V-IV. A NIRE in the SED of the star indicates the presence of circumstellar dust, which supports the assumption that KK Oph is a PMS Ae/Fe star. The star is a visual double star (Table B.1).

\section{C.36. SU Aur - HD 282624}

This star in the Aur T1 association was classified as G2 by CK79, F7-G2 III by Kh85 and G2 III by HB88. Since the star is a common proper-motion companion of $\mathrm{AB}$ Aur, we take the distance as determined by Hipparcos measurements for AB Aur $\left(d=144_{-20}^{+20} \mathrm{pc}\right.$; ATT97) and a foreground extinction $\boldsymbol{E}^{\text {is }}=0.12$ as determined for AB Aur by Felenbok et al. (1983). Bertout et al. (1988) give $i=40^{\circ}$. Independently Akeson et al. (2002) give $i=62^{\circ}$.

\section{C.38. HD 283572 - V987 Tau}

From the TCD we estimate a type G2-5, and from the highresolution spectroscopy in the visual and red, Walter et al. (1987) classify the star as G5 IV with $(B-V)_{0}^{\mathrm{J}}=0$. 77. The distance obtained by Hipparcos is $128_{-18}^{+26}$ pc (ESA 1997). The photometric variation is very small. At the largest brightness $V^{\mathrm{J}}=8.95$, $(B-V)^{\mathrm{J}}=0.80$ so that $\boldsymbol{E}^{\text {is }} \leq 0^{\mathrm{m}} 03$.

\section{C.39. $B D+09^{\circ} 880-H D 245185$}

This star was classified as A5 in the HD catalogue and quoted as such by HB88. Its visual spectrum was studied by

\footnotetext{
${ }^{9}$ Note that de Winter \& Thé (1990) obtained $d=300$ pc for A5 III, because they assumed $V_{\max }^{\mathrm{J}}=10^{\mathrm{m}} \cdot 32$ for KK Oph.
}

Penston et al. (1976) and FJ84. The 1992-photometry of the star (de Winter et al. 2001), however, indicates a type B9 in the TCD. The mis-classification was the reason why the first attempt to obtain a low-resolution UV spectrum of the star with IUE in 1984, was heavily overexposed in the SW-part $(\lambda>1275 \AA)$. The brightness $V^{\mathrm{J}}=9 \mathrm{~m} .93$, and monitoring of the star by the LTPV-group over several years has not revealed significant variability. Since the star is a member of the $\lambda$ Orionis association (Penston et al. 1976; Murdin \& Penston 1977) we have adopted $d \sim 450 \mathrm{pc}$ and $\boldsymbol{E}^{\text {is }} \sim 0$. 06 . Because of its low $E W_{\mathrm{Fe}}$ and its single $\mathrm{H} \alpha$-profile we assume that the star is viewed "pole-on" (see Appendix D.1 and D.3).

\section{C.40. HD $100546-K R$ Mus}

The star was identified as an Herbig B9 V emission-line star by $\mathrm{Hu}$ et al. (1989) and has a brightness $V^{\mathrm{J}}=6 \mathrm{~m}$.7. From the SED they derived $A_{\mathrm{v}}=0$. 20 which leads to $d=188 \mathrm{pc}$. However, from Hipparcos parallax measurements (ATT97) it follows that the distance of HD 100546 is much smaller: $103_{-6}^{+6} \mathrm{pc}$. Photometry of de Winter et al. (2001) shows no significant differences over 5 nights nor in comparison with the photometry by $\mathrm{Hu}$ et al. 1989. The high-resolution UV spectra of this star show several indications of infall of matter onto the star, e.g. stellar Ly $\alpha$-emission (Talavera et al. 1994) and variable red-shifted absorption components in the $\mathrm{Mg}$ II $\mathrm{h}, \mathrm{k}$ resonance lines and various Fe II lines (Grady et al. 1996). From the profile of the red-shifted Ly $\alpha$ emission-component we derived $i \sim 54^{\circ}$ (Appendix D.4). Recent coronographic NICMOS images at $1.6 \mu \mathrm{m}$ reveal an elliptical shape of the disc, extending to $380 \mathrm{AU}$ from the star (Augereau et al. 2001). From these observations they derived $i=51^{\circ}$.

\section{C.41. HD $98922-C D-52^{\circ} 4340$}

MBW98 classified this star as B9 V with a brightness $V^{\mathrm{J}}=6.77$. From photometry from 8 successive nights in 1992 by de Winter et al. (2001) we know that the star is not variable on a short timescale (days), and from the TCD we estimate $\boldsymbol{E}^{\text {is }} \leq 0$ m. 05 , so that with $M_{\mathrm{v}}=0.2$ this gives $d \approx 200 \mathrm{pc}$. However, Hipparcos measurements give a lower limit for the distance of 540 pc (AWT98) which is very uncertain as the observational range of Hipparcos is limited to $d \leq 350 \mathrm{pc}$. The star is probably a spectroscopic double-star (Bond 1970; de Groot 1971, Table B.1). Similar to HD 100546 its high-resolution Ly $\alpha$-profile shows indications for the presence of a red-shifted component (Talavera et al. 1994). From its shape we estimate $i \sim 45^{\circ}$ (Appendix D.4).

\section{C.42. HD $97048-$ CU Cha}

This B9.5 V star in the Cha T1 association was extensively studied (Rydgren 1980; Thé et al. 1986). There are no reports of significant variations in brightness of this $\operatorname{star}\left(V^{\mathrm{J}}=8 . \mathrm{m}^{4} 40-8 \mathrm{~m} .48\right)$ during the last 40 years (see e.g. de Winter et al. 2001). The distance derived from the Hipparcos parallax measurements (ATT97) is $180_{-20}^{+30} \mathrm{pc}$. The $\boldsymbol{E}^{\text {is }}$ can be estimated from that of HD 97240 (F5 V), which is close to the position of HD 97048. This star has no circumstellar excess (no NIR excess) and has $\boldsymbol{E}^{\text {is }}=0.05$ with $R^{\text {is }}=5.0$. If we assume that the foreground excess in that direction is proportional with distance, and that the distance and $\boldsymbol{E}^{\text {is }}$ of HD 97240 are $95 \mathrm{pc}$ and 0.05 , we conclude that $\boldsymbol{E}^{\text {is }} \sim 0$. 09 for HD 97048. 


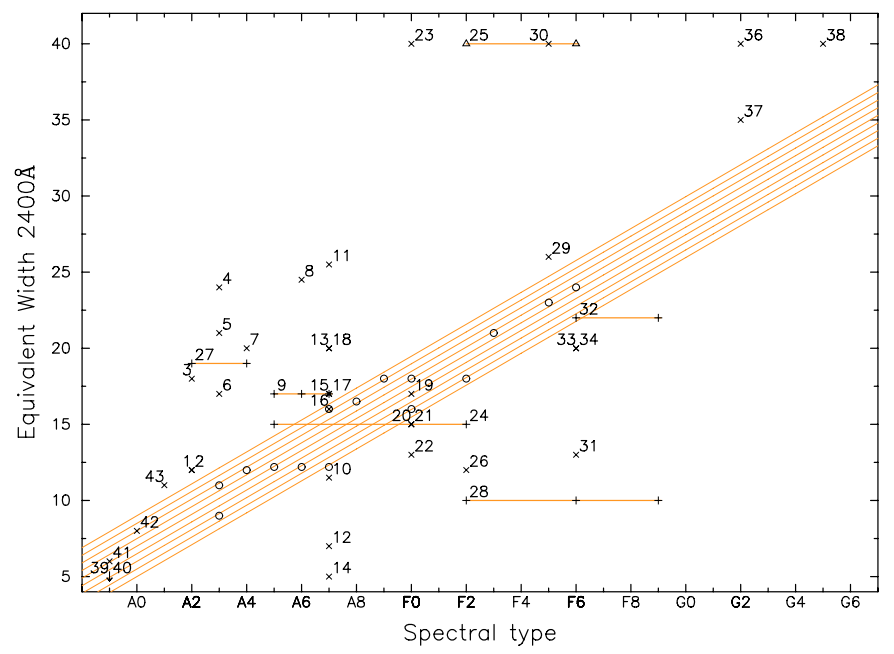

Fig. D.1. Equivalent width of the Fe II absorption feature $\left(E W_{\mathrm{Fe}}\right)$ versus spectral type of some program stars, indicated by $(X)$ or $(+)$ when the star covers a spectral range (horizontal line). The values of CQ Tau $(\triangle)$ are lower limits, while for $\mathrm{BD}+09^{\circ} 880$ and HD 100546 we only have an upper limit $(\downarrow)$. Open circles (o) denote comparison stars taken from the IUE spectral atlases (Appendix G.6).

\section{C.43. $H D 95881-C D-70^{\circ} 775$}

The MK classification of this star is A1-2 III-V and $V^{\mathrm{J}}=8.2$. Photometry of HD 95881 was given by MBW98, but photometric variability of this star is unknown to us. From the TCD MBW98 estimate $\boldsymbol{E}^{\text {is }}=0^{\mathrm{m}} \cdot 17$, so with $M_{\mathrm{v}}=0^{\mathrm{m}} \cdot 2$, this gives $d \approx$ $300 \mathrm{pc}$. On the basis of the $E W_{\mathrm{Fe}}$ and the $\mathrm{H} \alpha$-profile we assume that the system is seen "edge-on" (see Appendices D.1 and D.3).

\section{Appendix D: Indications for the disc inclination}

We use several observational sources in order to make an estimate of the inclination of the accretion disc of a star in our sample. None of these criteria are decisive in itself but together they may discriminate between "edge-on" and "pole-on" view (Sect. B.5).

\section{D.1. Table 1-Col. Y}

Gurzadyan \& Rustambekova (1980) found that the strength of the Fe II resonance absorption features in the UV spectrum of a shell star is increasing with the thickness of the gas envelope with a rate, depending on the spectral types of shell and star. If we apply this to the non-spherical envelope of an accretion disc, the $E W$ of the $2400 \AA$ Fe II absorption feature in the lowresolution UV spectrum of an accreting PMS Ae/Fe star should be correlated with the inclination in which we observe the disc. If we plot the $E W_{\mathrm{Fe}}$ (in $\AA$ for a bandwidth of $40 \AA$ ) of standard stars without shell (from the IUE spectral atlases) against their spectral types, we obtain a band (SSB hereafter, which is the lineair least square of the points plus 4 parallels on each side) that rises gradually for later spectral type (Fig. D.1).

The corresponding $E W$ 's of the PMS Ae/Fe stars of our sample (Table 1 Col. Y) are in general situated above the SSB, an indication for the presence of thick gas envelopes. The height of the points may be underestimated because of filling-in of the $2400 \AA$ absorption feature by Fe II emission from the accretion disc. However, for a few cases: NX Pup, V380 Ori, HK Ori, and $\mathrm{BD}+46^{\circ} 3471$, the $E W$ 's are even below the $\mathrm{SSB}$, which requires filling-in and which suggests that the corresponding envelopes are very thin in the direction of the observer. Since there are no indications that these stars have intrinsically much thinner gas envelopes than other Herbig stars of comparable spectral type (comparable SEDs with large IRE) it seems more probable that we observe these stars in a direction with low inclination, i.e. close to "pole-on". The number of such cases is small, in agreement with the expectation from the statistics of inclination angles.

\section{D.2. Table 1-Col. $X$}

The character of the photometric variability of the PMS Ae/Fe stars is another indicator for the inclination of the disc. Many of the stars in our sample were followed during photometric monitoring campaigns. A considerable amount of data has been secured by Kilkenny et al. (1985), Herbst et al. (1994), Zajtseva (1986), the LTPV group of ESO observers (1986-1996), the ROTOR-group (Taskent), the Hipparcos and Tycho projects (1997), and several other groups. The photometric data of the PMS Ae/Fe stars in our sample show several types of variability in the TCD. We distinguish the following types (Sect. B.5):

r. a large group of stars (including the $U X O R \mathrm{~s}^{2}$ ), which show strong variations (up to 2 or $3 \mathrm{mag}$ ) along the reddening direction of the TCD. Examples are: XY Per, WW Vul, UX Ori, BF Ori, HR 5999, HD 142666, V346 Ori, V350 Ori, HD 35929, R CrA, CQ Tau, AK Sco, NV Ori, RR Tau, KK Oph, SU Aur, RY Tau, and perhaps Z CMa. This variability is characteristic for a variable circumstellar dust extinction, which can result when we observe a rotating disc envelope with non-isotropic dust column density more or less "edge-on". For these stars the variations in $(U-B)^{\mathrm{J}}$ are approximately equal to those in $(B-V)^{\mathrm{J}}$. During deep brightness minima several of these stars (UX Ori, BF Ori, WW Vul, CQ Tau, and RR Tau) show declines in $(U-B)^{\mathrm{J}}$ ("blueing", Fig. D.2) together with sudden, strong rises in linear polarisation. These "blueing" and polarisation excursions can be explained by the contribution of a fine-dust halo, which becomes dominant during the obscuration of the direct stellar light by very dense circumstellar clouds in the disc (Grinin 1988). The model for this type of variations works if the disc is observed more or less "edge-on" $\left(i>60^{\circ}\right)$.

It may be noted that three stars, which are still far from the MS, lost their c.s. dust in a sudden event some time ago: FU Ori (1936), BN Ori (1945), and V351 Ori (1987) and are indicated with $\dagger$ in Table 1 . The latter two stars originally behaved photometrically as the stars of type $r$, but should at present be classified as belonging to type $n$. FU Ori probably behaved similarly, but no data is available from before the event. BD+46 3471 and HD 35929 are also far from the MS and show small brightness variations, but recent episodes of c.s. dust dissipation have not been observed.

o. a small class of stars which shows strong "blueing" variations for moderate variations of $V^{\mathrm{J}}\left(0.1-1 . \mathrm{m}^{\mathrm{m}} 0\right)$. They rarely vary in the reddening direction. In the TCD their variations in $(U-B)^{\mathrm{J}}$ are $2-10$ times larger than those in $(B-V)^{\mathrm{J}}$. Examples are: BD $+24^{\circ} 676$, NX Pup, HK Ori, Z CMa, V380 Ori, and HD 283572 (Fig. D.3). We suggest that the strong $U$-band variations are correlated with a strong, variable excess in the SW-part of the spectra, which is contributed by an instable BL and hot inner disc of these stars (Sect. A.5). These contributions are probably only detectable when the system is observed approximately 
P. F. C. Blondel and H. R. E. Tjin A Djie: Modeling of PMS Ae/Fe stars using UV spectra, Online Material p 26
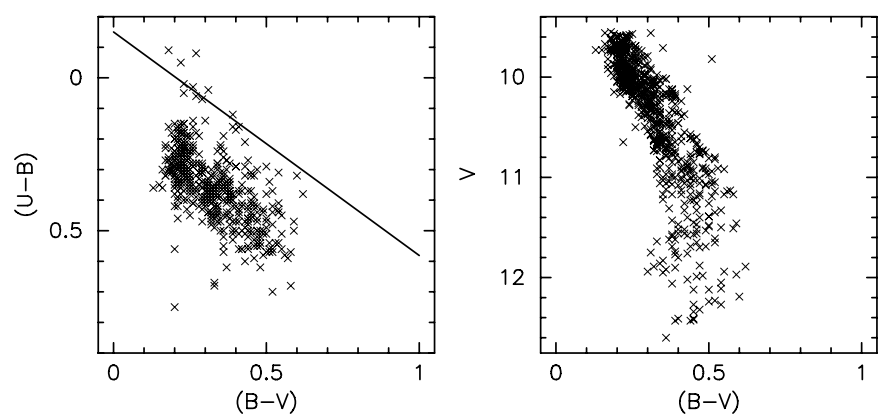

Fig. D.2. TCD \& CMD of BF Ori (type $r$ ) based on data from sun. astro.wesleyan. edu/pub/ttauri, Herbst et al. 1994.
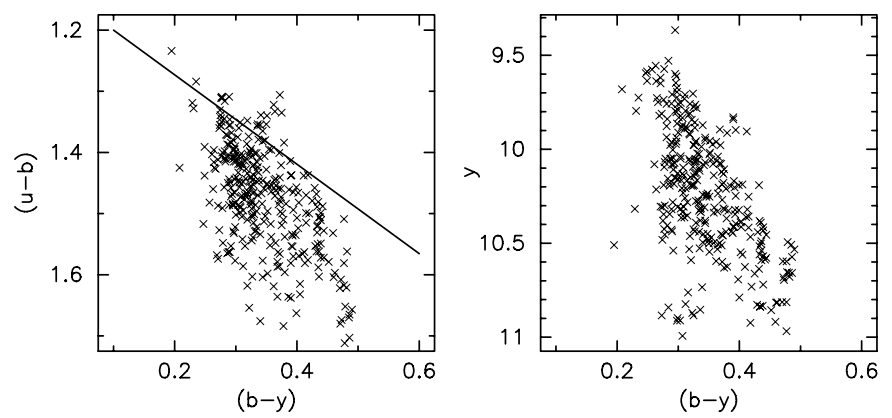

Fig. D.3. TCD \& CMD of NX Pup (type o) based on data from the LTPV group (ESO SR-8, SR-12, SR-14, \& SR-16).
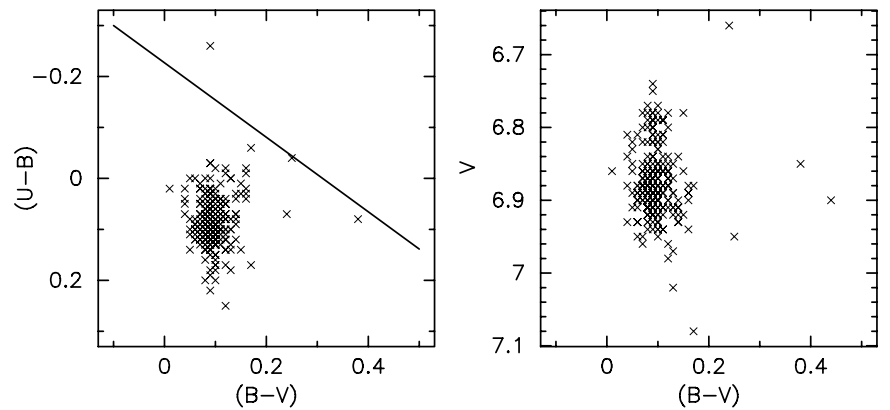

Fig. D.4. TCD \& CMD of HD 163296 (type $n$ ) based on data from sun. astro. wesleyan. edu/pub/ttauri, Herbst et al. 1994.

"pole-on" $\left(i \leq 20^{\circ}\right)$. Rare variations in the reddening direction may be due to c.s. dust clouds moving parallel to, but outside the disc plane.

$\mathrm{n}$. the remaining stars of our sample show only very small $(<0$. 1$)$ variations in brightness: HD 163296, HD 150193, HD 31648, $\beta$ Pic, HD 104237, HD 169142, HD 36112, HD 319614, HD 144432, BD $+47^{\circ} 4206, \mathrm{BD}+09^{\circ} 880$, HD 100546, and HD 97048 (Fig. D.4). The amplitudes of the variations of this group are probably too small to discriminate between high and low inclinations. Most stars of this group have evolved to the neighbourhood of the ZAMS and have not much circumstellar dust any more (Sect. 6.3), so that photometric effects due to variations in c.s. dust column density cannot be expected.

The line in Figs. D.2-D.4 indicates the $R=3.1$ reddening-line. Stars for which no photometry is available are BD $+46^{\circ} 3471$, HD 199143, HD 98922, and HD 95881, so it is not possible to contain them in one of the previous groups and are indicated with $(-)$.
Table D.1. Review of inclination angles (in degrees) from Ly $\alpha$ emission-line profiles, see Blondel et al. (1993) for details.

\begin{tabular}{|c|c|c|c|c|c|c|c|}
\hline \multirow[b]{2}{*}{ star } & \multicolumn{3}{|c|}{ case } & \multirow[b]{2}{*}{ star } & \multicolumn{3}{|c|}{ case } \\
\hline & $\bar{A}$ & $\mathrm{C}$ & $\overline{\mathrm{D}}$ & & $\bar{A}$ & $\mathrm{C}$ & $\bar{D}$ \\
\hline HD 163296 & 67 & 57 & - & HD 150193 & - & 67 & - \\
\hline HR 5999 & 53 & 53 & - & HD 98922 & 53 & 45 & - \\
\hline HD 104237 & - & 53 & 53 & HD 100546 & - & 53 & - \\
\hline
\end{tabular}

\section{D.3. Table 1-Col. Z}

Another diagnostic tool for the inclination of the disc is the shape of the $\mathrm{H} \alpha$-profile. In a sample of $63 \mathrm{PMS} \mathrm{Be} / \mathrm{Ae} / \mathrm{Fe}$ stars Grinin $\&$ Rostopchina (1996) noted a correlation between the type of $\mathrm{H} \alpha$-profile and the amplitude of the photometric variability of a Herbig star:

(1) of stars with large amplitude $\left(\Delta V^{\mathrm{J}}>1 \mathrm{~m}\right.$. $)$ the majority $(73 \%)$ has a 2-component $\mathrm{H} \alpha$-profile, $13 \%$ has a P-Cygni-profile, while only $7 \%$ has a single-profile.

(2) for the stars with smaller amplitude $\left(\Delta V^{\mathrm{J}}<0 . \mathrm{m} 6\right) 42 \%$ has a 2 -component profile, $33 \%$ has a single-profile, and $20 \%$ has a P-Cygni-profile.

In Table 1 we have used the profile classification scheme of Reipurth et al. (1996):

I. single, symmetric profiles;

II. double profiles with $<50 \%$ difference in peak intensity;

III. double profiles with $>50 \%$ difference in peak intensity;

IV. P-Cygni profiles.

The profile types are further specified by B or R, according to the position of the stronger component (blue or red side). From Table 1 we note that almost all stars with photometric variability type $r$ have class II or III H $\alpha$-profiles, whereas the stars with variability type $o$ : V380 Ori, HK Ori, and NX Pup have profiles of type I or II. The general correlation between $\mathrm{H} \alpha$-profile type and variability type suggests that we can also use the $\mathrm{H} \alpha$-profile type to indicate the inclination for those stars in Table 1 that do not show significant photometric variability (type $n$ ). However, it should be kept in mind that the correlation, noted by Grinin and Rostopchina, is only valid for $75 \%$ of the stars (NX Pup and V346 Ori belong to the other 25\%), so that this method for estimating the inclination has some intrinsic uncertainty.

\section{D.4. Stellar Lyman $\alpha$-emission}

In a few cases we have observed the Ly $\alpha$-line profiles in highresolution with IUE (Table D.1). By fitting the shapes of the profiles of these lines we could determine the inclination of these cases (Blondel et al. 1993; Talavera et al. 1994), provided that the $\operatorname{Ly} \alpha$-emission is produced by infalling ions. The results for HD 163296, HR 5999, HD 104237, HD 150193, HD 98922, and HD 100546 agree with those derived with the $E W_{\mathrm{Fe}}$-method (Sect D.1). These stars appear to have large inclinations $\left(i \approx 60^{\circ}\right)$. With IUE the Ly $\alpha$-method is limited to those stars that were bright enough to show detectable stellar Ly $\alpha$ emission in the SW-camera. Stellar Ly $\alpha$-emission is confirmed by Hubble observations of HD 104237 (Brown et al. 1997) and HD 163296 (Devine et al. 2000). 
P. F. C. Blondel and H. R. E. Tjin A Djie: Modeling of PMS Ae/Fe stars using UV spectra, Online Material p 27

Appendix E: ZAMS ages (Table 8)

Table E.1. $t_{\text {ZAMS }}$ values Bernasconi (1996), see also Fig. 43.

\begin{tabular}{llll}
\hline \hline$\frac{M_{\star}}{M_{\odot}}$ & $\log \frac{L_{\star}}{L_{\odot}}$ & $\log \frac{T_{\text {eff }}}{\mathrm{K}}$ & $\log \frac{t}{\mathrm{yr}}$ \\
\hline 1.0 & - & 3.75 & - \\
1.25 & 0.43 & 3.81 & 7.47 \\
1.5 & 0.73 & 3.87 & 7.27 \\
1.7 & 1.00 & 3.92 & 7.10 \\
2.0 & 1.30 & 3.975 & 6.90 \\
2.5 & 1.70 & 4.05 & 6.60 \\
3.0 & 2.04 & 4.10 & 6.35 \\
4.0 & 2.40 & 4.18 & 6.00 \\
\hline
\end{tabular}

Table E.2. $t_{\text {ZAMS }}$ values Siess B (1999).

\begin{tabular}{clll}
\hline \hline$\frac{M_{\star}}{M_{\odot}}$ & $\log \frac{L_{\star}}{L_{\odot}}$ & $\log \frac{T_{\text {eff }}}{\mathrm{K}}$ & $\log \frac{t}{\mathrm{yr}}$ \\
\hline 0.6 & 0.07 & 3.772 & 7.75 \\
0.7 & 0.25 & 3.792 & 7.66 \\
0.8 & 0.43 & 3.812 & 7.57 \\
0.9 & 0.57 & 3.832 & 7.48 \\
1.0 & 0.70 & 3.855 & 7.41 \\
1.1 & 0.83 & 3.880 & 7.33 \\
1.3 & 1.05 & 3.92 & 7.17 \\
1.4 & 1.14 & 3.942 & 7.12 \\
1.5 & 1.24 & 3.960 & 7.05 \\
1.7 & 1.40 & 3.994 & 6.95 \\
2.0 & 1.60 & 4.032 & 6.81 \\
2.2 & 1.75 & 4.057 & 6.71 \\
2.5 & 1.90 & 4.085 & 6.60 \\
3.0 & 2.14 & 4.138 & 6.44 \\
4.0 & 2.54 & 4.208 & 6.16 \\
\hline
\end{tabular}

Table E.3. $t_{\text {ZAMS }}$ values Siess NG (2000).

\begin{tabular}{llll}
\hline \hline$\frac{M_{\star}}{M_{\odot}}$ & $\log \frac{L_{\star}}{L_{\odot}}$ & $\log \frac{T_{\text {eff }}}{\mathrm{K}}$ & $\log \frac{t}{\mathrm{yr}}$ \\
\hline 1.1 & 0.0 & 3.75 & 7.76 \\
1.2 & 0.20 & 3.77 & 7.65 \\
1.3 & 0.37 & 3.79 & 7.55 \\
1.4 & 0.52 & 3.81 & 7.46 \\
1.5 & 0.67 & 3.833 & 7.37 \\
1.6 & 0.79 & 3.860 & 7.29 \\
1.7 & 0.91 & 3.885 & 7.22 \\
1.8 & 1.01 & 3.91 & 7.16 \\
1.9 & 1.11 & 3.93 & 7.10 \\
2.0 & 1.20 & 3.95 & 7.04 \\
2.2 & 1.37 & 3.98 & 6.93 \\
2.5 & 1.59 & 4.02 & 6.81 \\
2.7 & 1.72 & 4.045 & 6.73 \\
3.0 & 1.90 & 4.077 & 6.62 \\
3.5 & 2.15 & 4.118 & 6.46 \\
4.0 & 2.35 & 4.158 & 6.33 \\
5.0 & 2.71 & 4.216 & 6.0625 \\
\hline
\end{tabular}

Appendix F: IUE images - PMS stars.

Table F.1.

\begin{tabular}{|c|c|c|c|c|c|}
\hline code & star & dd-mm-yy & image & exp. & note \\
\hline $01-01$ & XY Per & $19-09-95$ & 355968 & 6000 & \\
\hline 01-01 & XY Per & $19-09-95$ & 131507 & 1320 & $* 1.3$ \\
\hline 02-01 & WW Vul & $16-07-93$ & 348123 & 7200 & \\
\hline 02-01 & WW Vul & $16-07-93$ & 125929 & 2100 & \\
\hline 02-02 & WW Vul & $20-09-93$ & 348692 & 9000 & \\
\hline 02-02 & WW Vul & $20-09-93$ & 126423 & 2100 & \\
\hline $02-03$ & WW Vul & 3-09-95 & 355804 & 7200 & \\
\hline $02-03$ & WW Vul & 3-09-95 & 131401 & 1200 & \\
\hline $02-.$. & WW Vul & $20-09-95$ & 131512 & 2820 & \\
\hline 03-01 & UX Ori & $29-08-81$ & 314857 & 8820 & \\
\hline 03-01 & UX Ori & & 129212 & & 03-03 \\
\hline 03-02 & UX Ori & $30-10-83$ & 321406 & 600 & \\
\hline 03-02 & UX Ori & $30-10-83$ & 102179 & 600 & \\
\hline 03-03 & UX Ori & 21-09-94 & 352168 & 1500 & \\
\hline 03-03 & UX Ori & 21-09-94 & 129212 & 900 & \\
\hline 03-04 & UX Ori & $8-10-93$ & 348870 & 1200 & \\
\hline 03-04 & UX Ori & $8-10-93$ & 126528 & 720 & \\
\hline 03-05 & UX Ori & $9-01-84$ & 321976 & 1800 & \\
\hline 03-05 & UX Ori & $9-01-84$ & 102585 & 600 & \\
\hline 03-06 & UX Ori & $1-02-90$ & 338105 & 1800 & \\
\hline 03-06 & UX Ori & & 109519 & & $03-08$ \\
\hline 03-07 & UX Ori & $1-04-83$ & 319605 & 600 & \\
\hline 03-07 & UX Ori & $1-04-83$ & 215639 & 900 & \\
\hline 03-.. & UX Ori & $1-04-83$ & 215640 & 1200 & \\
\hline 03-08 & UX Ori & $14-11-86$ & 329676 & 3000 & \\
\hline 03-08 & UX Ori & $14-11-86$ & 109519 & 2800 & \\
\hline 03-09 & UX Ori & $18-11-93$ & 349253 & 5400 & \\
\hline 03-09 & UX Ori & $18-11-93$ & 126772 & 2100 & \\
\hline $03-10$ & UX Ori & $25-09-92$ & 345774 & 10800 & \\
\hline $03-10$ & UX Ori & $24-09-92$ & 123996 & 2880 & \\
\hline $03-11$ & UX Ori & $5-02-92$ & 343931 & 5400 & \\
\hline 03-11 & UX Ori & $5-02-92$ & 122337 & 1320 & \\
\hline $03-.$. & UX Ori & $11-09-94$ & 352088 & 1200 & \\
\hline $03-.$. & UX Ori & $11-09-94$ & 129151 & 900 & \\
\hline 04- & HD 163296 & See Table F. & & & \\
\hline $05-.$. & HD 150193 & $12-04-93$ & 347468 & 3600 & \\
\hline 05-01 & HD 150193 & $12-04-93$ & 347469 & 5400 & \\
\hline 05-01 & HD 150193 & $12-04-93$ & 125327 & 360 & \\
\hline 06-01 & $\mathrm{BD}+24^{\circ} 676$ & $19-09-95$ & 355967 & 7199 & \\
\hline 06-01 & $\mathrm{BD}+24^{\circ} 676$ & 19-09-95 & 131506 & 1800 & \\
\hline 07-.. & BF Ori & 7-09-82 & 317863 & 22200 & \\
\hline $07-.$. & BF Ori & 7-09-82 & 214105 & 1800 & \\
\hline 07-01 & BF Ori & $1-01-82$ & 315920 & 5400 & \\
\hline 07-01 & BF Ori & $1-01-82$ & 212255 & 2100 & \\
\hline 07-02 & BF Ori & 10-09-91 & 342439 & 180 & $*$ \\
\hline 07-02 & BF Ori & $19-10-89$ & 116572 & 480 & $*$ \\
\hline $07-.$. & BF Ori & $19-10-89$ & 116570 & 1560 & \\
\hline 08-01 & HD 31648 & $15-04-79$ & 304949 & 1800 & \\
\hline 08-01 & HD 31648 & $15-04-79$ & 204278 & 120 & \\
\hline 08-02 & HD 31648 & $16-04-79$ & 304950 & 300 & \\
\hline 08-02 & HD 31648 & $16-04-79$ & 204279 & 420 & \\
\hline 08-03 & HD 31648 & $10-09-91$ & 342438 & 3600 & \\
\hline 08-03 & HD 31648 & & 204278 & & 08-01 \\
\hline
\end{tabular}


Table F.2.

\begin{tabular}{|c|c|c|c|c|c|}
\hline code & star & cdd-mm-yy & image & exp. & note \\
\hline $09-01$ & HR 5999 & $8-07-85$ & 326372 & 720 & \\
\hline 09-01 & HR 5999 & $7-07-85$ & 106364 & 60 & \\
\hline 09-. & HR 5999 & $7-07-85$ & 326369 & 240 & \\
\hline 09-02 & HR 5999 & 18-09-91 & 342497 & 600 & \\
\hline 09-02 & HR 5999 & $18-09-91$ & 121267 & 120 & \\
\hline 09-03 & HR 5999 & $31-08-80$ & 309973 & 720 & \\
\hline 09-03 & HR 5999 & & 121267 & & 09-02 \\
\hline 09-04 & HR 5999 & $18-05-78$ & 301569 & 1080 & \\
\hline 09-04 & HR 5999 & $18-05-78$ & 201511 & 480 & \\
\hline 09-05 & HR 5999 & $11-09-83$ & 321020 & 1560 & \\
\hline 09-05 & HR 5999 & $11-09-83$ & 216768 & 480 & (1) \\
\hline 09-05 & HR 5999 & & 216768 & 180 & $\mathrm{SAP} * 2.3$ \\
\hline 09-06 & HR 5999 & $31-08-83$ & 320861 & 360 & \\
\hline 09-06 & HR 5999 & $31-08-83$ & 216706 & 180 & \\
\hline 09-.. & HR 5999 & $31-08-83$ & 320863 & 2400 & \\
\hline 09-.. & HR 5999 & $31-08-83$ & 216707 & 720 & \\
\hline 09-07 & HR 5999 & $6-08-86$ & 328853 & 1800 & \\
\hline 09-07 & HR 5999 & & 216706 & & 09-06 \\
\hline 09-08 & HR 5999 & $12-10-83$ & 321276 & 666 & \\
\hline 09-08 & HR 5999 & $12-10-83$ & 216970 & 215 & \\
\hline 09-09 & HR 5999 & $2-06-83$ & 320133 & 1080 & \\
\hline 09-09 & HR 5999 & & 216970 & & 09-08 \\
\hline $09-10$ & HR 5999 & $8-03-85$ & 325390 & 1800 & \\
\hline $09-10$ & HR 5999 & & 214027 & & $09-12$ \\
\hline $09-11$ & HR 5999 & $7-03-85$ & 325379 & 2100 & \\
\hline $09-11$ & HR 5999 & & 214027 & & $09-12$ \\
\hline 09-.. & HR 5999 & $27-08-82$ & 317789 & 1800 & \\
\hline 09-12 & HR 5999 & $28-08-82$ & 317790 & 720 & \\
\hline 09-12 & HR 5999 & $28-08-82$ & 214027 & 480 & \\
\hline $10-01$ & $\beta$ Pic & $5-11-84$ & 324392 & 22 & \\
\hline $10-01$ & $\beta$ Pic & $5-11-84$ & 217531 & 13 & \\
\hline $11-01$ & HD 104237 & $5-08-89$ & 116073 & 60 & \\
\hline $11-01$ & HD 104237 & $5-08-89$ & 336799 & 600 & (2) \\
\hline $11-01$ & HD 104237 & $5-08-89$ & 336799 & 240 & SAP $* 2.5$ \\
\hline $11-02$ & HD 104237 & $6-05-91$ & 341588 & 540 & \\
\hline $11-02$ & HD 104237 & & 116073 & & $11-01$ \\
\hline $11-.$. & HD 104237 & 7-05-91 & 341590 & 900 & \\
\hline $11-03$ & HD 104237 & $12-06-91$ & 341822 & 900 & \\
\hline $11-03$ & HD 104237 & & 116073 & & $11-01$ \\
\hline $12-01$ & HD 169142 & $11-08-95$ & 355483 & 420 & $* 1.15$ \\
\hline $12-01$ & HD 169142 & $11-08-95$ & 131285 & 190 & \\
\hline $13-01$ & HD 36112 & $19-02-95$ & 353939 & 720 & \\
\hline $13-01$ & HD 36112 & $19-02-95$ & 130023 & 120 & \\
\hline $14-01$ & HD 139614 & $8-04-95$ & 354332 & 600 & \\
\hline $14-01$ & HD 139614 & 8-04-95 & 130412 & 240 & \\
\hline $15-01$ & HD 142666 & $7-08-88$ & 334054 & 6000 & \\
\hline $15-01$ & HD 142666 & $7-08-88$ & 113800 & 900 & \\
\hline $16-01$ & V346 Ori & $22-09-95$ & 355993 & 5400 & \\
\hline $16-01$ & V346 Ori & $22-09-95$ & 131521 & 1200 & \\
\hline $17-01$ & V350 Ori & 23-01-94 & 349877 & 8400 & \\
\hline $17-01$ & V350 Ori & 24-01-94 & 127279 & 4200 & \\
\hline $18-01$ & V351 Ori & $5-02-92$ & 343932 & 4200 & \\
\hline $18-01$ & V351 Ori & $5-02-92$ & 122338 & 900 & \\
\hline $18-02$ & V351 Ori & $14-11-86$ & 329678 & 3840 & \\
\hline $18-02$ & V351 Ori & $14-11-86$ & 109520 & 1500 & \\
\hline
\end{tabular}

Table F.3.

\begin{tabular}{|c|c|c|c|c|c|}
\hline code & star & "dd-mm-yy & $\overline{\text { image }}$ & $\overline{\text { exp. }}$ & $\overline{\text { note }}$ \\
\hline $19-01$ & BN Ori & $22-01-84$ & 322075 & 10800 & \\
\hline $19-01$ & BN Ori & $22-01-84$ & 102694 & 720 & \\
\hline $19-.$. & BN Ori & $22-01-84$ & 102693 & 1800 & \\
\hline $19-02$ & BN Ori & $11-10-86$ & 329420 & 7200 & \\
\hline $19-02$ & BN Ori & $11-10-86$ & 109294 & 600 & \\
\hline $19-.$. & BN Ori & $11-11-86$ & 329657 & 12600 & \\
\hline $19-03$ & BN Ori & $13-11-86$ & 329660 & 11400 & * \\
\hline $19-03$ & BN Ori & $11-11-86$ & 109508 & 600 & $*$ \\
\hline $20-01$ & HD 35929 & $12-01-94$ & 349824 & 1200 & \\
\hline $20-01$ & HD 35929 & $12-01-94$ & 127222 & 180 & \\
\hline $20-02$ & HD 35929 & 23-01-94 & 349876 & 5400 & \\
\hline $20-02$ & HD 35929 & & 127222 & & $20-01$ \\
\hline $20-.$. & HD 35929 & $12-11-95$ & 356191 & 10800 & \\
\hline $20-.$. & HD 35929 & $13-11-95$ & 131695 & 540 & \\
\hline 21-01 & HD 144432 & $23-03-90$ & 338425 & 1800 & \\
\hline 21-01 & HD 144432 & $24-03-90$ & 117586 & 180 & \\
\hline $21-02$ & HD 144432 & $20-08-90$ & 339496 & 1800 & * \\
\hline $21-02$ & HD 144432 & $31-03-95$ & 130354 & 600 & $*(3)$ \\
\hline $21-02$ & HD 144432 & $9-03-95$ & 130188 & 2100 & * \\
\hline $21-02$ & HD 144432 & $23-03-90$ & 117585 & 600 & * \\
\hline $22-01$ & $\mathrm{BD}+47^{\circ} 4206$ & $11-08-96$ & 357779 & 7200 & \\
\hline $22-01$ & $\mathrm{BD}+47^{\circ} 4206$ & $11-08-96$ & 132556 & 540 & \\
\hline 23-01 & FU Ori & $5-03-79$ & 304495 & 3600 & \\
\hline 23-01 & FU Ori & $5-03-79$ & 203933 & 3600 & \\
\hline $23-.$. & FU Ori & $13-08-82$ & 213935 & 1500 & \\
\hline $23-.$. & FU Ori & $13-08-82$ & 213936 & 2400 & \\
\hline $23-.$. & FU Ori & $13-08-82$ & 213939 & 210 & \\
\hline $23-02$ & FU Ori & $13-08-82$ & 317674 & 24600 & $*$ \\
\hline $23-02$ & FU Ori & $31-08-83$ & 216704 & 21300 & $*$ \\
\hline $23-02$ & FU Ori & $31-08-83$ & 216705 & 2100 & (4) \\
\hline $23-.$. & FU Ori & $31-08-83$ & 216705 & 2100 & \\
\hline 24-01 & $\mathrm{R}$ CrA & $20-03-82$ & 316589 & 13200 & \\
\hline 24-01 & $\mathrm{R}$ CrA & $20-03-82$ & 212825 & 5100 & \\
\hline $25-.$. & CQ Tau & $8-04-86$ & 328124 & 10800 & \\
\hline $25-.$. & CQ Tau & $8-04-86$ & 107973 & 1200 & \\
\hline $25-01$ & CQ Tau & $11-11-86$ & 329659 & 19800 & \\
\hline $25-01$ & CQ Tau & $12-11-86$ & 109510 & 1200 & $* 1.25$ \\
\hline $25-02$ & CQ Tau & $12-10-86$ & 329427 & 6000 & \\
\hline $25-02$ & CQ Tau & $12-10-86$ & 109310 & 600 & $* 1.25$ \\
\hline $25-.$. & CQ Tau & $20-09-95$ & 355974 & 18900 & \\
\hline $25-$. & CQ Tau & $20-09-95$ & 131511 & 3600 & \\
\hline $26-01$ & NX Pup & $27-08-82$ & 317788 & 6600 & \\
\hline $26-01$ & NX Pup & $27-08-82$ & 214025 & 900 & \\
\hline $26-02$ & NX Pup & $7-10-82$ & 318236 & 7800 & \\
\hline $26-02$ & NX Pup & $7-10-82$ & 214350 & 600 & \\
\hline $26-03$ & NX Pup & $16-04-94$ & 350565 & 3000 & \\
\hline $26-03$ & NX Pup & $16-04-94$ & 127913 & 720 & \\
\hline $27-01$ & $\mathrm{BD}+46^{\circ} 3471$ & $26-01-81$ & 311158 & 7740 & $*$ \\
\hline 27-01 & $\mathrm{BD}+46^{\circ} 3471$ & $24-01-82$ & 212418 & 1800 & $*$ \\
\hline $28-01$ & HK Ori & $30-01-84$ & 322143 & 9900 & \\
\hline $28-01$ & HK Ori & $30-01-84$ & 102718 & 3000 & \\
\hline $28-.$. & HK Ori & $8-10-93$ & 348868 & 7200 & \\
\hline $28-.$. & HK Ori & $8-10-93$ & 126525 & 1800 & \\
\hline 29-01 & AK Sco & $29-08-82$ & 317804 & 10200 & \\
\hline 29-01 & AK Sco & $29-08-82$ & 214048 & 720 & \\
\hline $29-02$ & AK Sco & $1-04-88$ & 333197 & 10500 & \\
\hline $29-02$ & AK Sco & $1-04-88$ & 112966 & 900 & \\
\hline $29-03$ & AK Sco & $9-04-88$ & 333241 & 11100 & \\
\hline $29-03$ & AK Sco & $9-04-88$ & 113009 & 720 & \\
\hline
\end{tabular}


P. F. C. Blondel and H. R. E. Tjin A Djie: Modeling of PMS Ae/Fe stars using UV spectra, Online Material p 29

Table F.4.

\begin{tabular}{llrrrl}
\hline \hline code & star & dd-mm-yy & image & exp. & note \\
\hline $30-01$ & Z CMa & $17-04-79$ & 304961 & 8700 & \\
$30-01$ & Z CMa & $17-04-79$ & 204293 & 4200 & \\
$30-.$. & Z CMa & $15-04-88$ & 113049 & 2400 & \\
$30-02$ & Z CMa & $19-03-88$ & 333112 & 2940 & \\
$30-02$ & Z CMa & $19-03-88$ & 112885 & 1800 & \\
$31-01$ & HD 199143 & $25-06-95$ & 355115 & 3300 & \\
$31-01$ & HD 199143 & $25-06-95$ & 130948 & 70 & \\
$31-02$ & HD 199143 & $3-07-95$ & 355194 & 3600 & \\
$31-02$ & HD 199143 & $3-07-95$ & 130979 & 80 & \\
$32-01$ & V380 Ori & $17-04-79$ & 304960 & 3600 & \\
$32-01$ & V380 Ori & $17-04-79$ & 204291 & 720 & \\
$32-.$. & V380 Ori & $19-10-89$ & 337392 & 2400 & \\
$32-.$. & V380 Ori & $19-10-89$ & 116571 & 1020 & \\
$33-01$ & NV Ori & $8-04-86$ & 328123 & 9000 & \\
$33-01$ & NV Ori & $8-04-86$ & 107972 & 1200 & \\
$33-.$. & NV Ori & $16-04-81$ & 210370 & 1800 & \\
$34-.$. & RR Tau & $22-01-84$ & 322076 & 5220 & \\
$34-.$. & RR Tau & $7-10-93$ & 126522 & 1800 & \\
$34-01$ & RR Tau & $8-10-93$ & 348867 & 10800 & \\
$34-01$ & RR Tau & $8-10-93$ & 126524 & 3600 & \\
$35-01$ & KK Oph & $18-08-94$ & 351845 & 6900 & $*$ \\
$35-01$ & KK Oph & $16-07-93$ & 125932 & 6600 & $*$ \\
$36-01$ & SU Aur & $12-11-80$ & 310591 & 10800 & \\
$36-01$ & SU Aur & $12-11-80$ & 209282 & 1800 & \\
$37-01$ & RY Tau & $28-10-79$ & 307034 & 10800 & \\
$37-01$ & RY Tau & $28-10-79$ & 205963 & 1200 & $*$ \\
$38-01$ & HD 283572 & $9-10-82$ & 318244 & 26400 & $*$ \\
$38-01$ & HD 283572 & $10-10-83$ & 216961 & 1200 & $*$ \\
$39-.$. & BD+09 880 & $21-01-84$ & 322064 & 9480 & \\
$39-01$ & BD+09 880 & $22-02-94$ & 350084 & 1035 & \\
$39-01$ & BD+09 880 & $22-02-94$ & 127467 & 470 & \\
$39-.$. & BD+09 880 & $22-09-95$ & 131522 & 1200 & \\
$40-01$ & HD 100546 & $12-05-95$ & 354658 & 120 & SAP*2.5 \\
$40-01$ & HD 100546 & $12-05-95$ & 130679 & 40 & 5 \\
$40-01$ & HD 100546 & $12-05-95$ & 130679 & 30 & SAP*2.5 \\
$41-01$ & HD 98922 & $14-11-82$ & 318553 & 180 & \\
$41-01$ & HD 98922 & $14-11-82$ & 214624 & 60 & \\
$42-01$ & HD 97048 & $27-02-80$ & 308066 & 720 & \\
$42-01$ & HD 97048 & $27-02-80$ & 107032 & 270 & $*$ \\
$43-01$ & HD 95881 & $25-05-95$ & 354755 & 900 & $*$ \\
$43-01$ & HD 95881 & $16-07-93$ & 125931 & 154 & $*$ \\
\hline & & & & & \\
\hline
\end{tabular}

Table F.5. The (sparse) LW-images marked with $* *$ are used for a range of SW-images to complete the spectrum.

\begin{tabular}{|c|c|c|c|c|c|}
\hline$\overline{c \text { code }}$ & star & "dd-mm-yy & $\overline{\text { image }}$ & exp. & \\
\hline $04-11$ & HD 163296 & $13-08-78$ & 302283 & 54.8 & \\
\hline 04-11 & HD 163296 & $13-08-78$ & 202065 & 29.8 & \\
\hline 04-12 & HD 163296 & $14-04-79$ & 304936 & 150 & \\
\hline 04-12 & HD 163296 & $14-04-79$ & 204264 & 39.6 & \\
\hline 04-13 & HD 163296 & $19-09-79$ & 306566 & 149 & \\
\hline $04-13$ & HD 163296 & $19-09-79$ & 205626 & 38.8 & \\
\hline $04-6 a$ & HD 163296 & $29-07-86$ & 328778 & 1080 & \\
\hline $04-6 b$ & HD 163296 & $30-07-86$ & 328782 & 480 & \\
\hline $04-6 c$ & HD 163296 & $30-07-86$ & 328788 & 1080 & \\
\hline 04-6d & HD 163296 & $31-07-86$ & 328791 & 1080 & \\
\hline $04-6 \mathrm{e}$ & HD 163296 & $31-07-86$ & 328792 & 630 & \\
\hline $04-6 f$ & HD 163296 & $1-08-86$ & 328802 & 1080 & \\
\hline $04-6 \mathrm{~g}$ & HD 163296 & $1-08-86$ & 328805 & 480 & \\
\hline $04-6 \mathrm{~h}$ & HD 163296 & $1-08-86$ & 328810 & 1080 & \\
\hline $04-6 \mathrm{i}$ & HD 163296 & $1-08-86$ & 328811 & 300 & \\
\hline $04-6 j$ & HD 163296 & $2-08-86$ & 328813 & 480 & \\
\hline $04-6 \mathrm{k}$ & HD 163296 & $2-08-86$ & 328816 & 120 & \\
\hline $04-61$ & HD 163296 & $3-08-86$ & 328818 & 480 & \\
\hline $04-6 \mathrm{~m}$ & HD 163296 & $3-08-86$ & 328824 & 150 & \\
\hline $04-6 n$ & HD 163296 & $4-08-86$ & 328827 & 480 & \\
\hline $04-6 o$ & HD 163296 & $4-08-86$ & 328833 & 1080 & \\
\hline $04-6 p$ & HD 163296 & $5-08-86$ & 328837 & 480 & \\
\hline $04-6 p$ & HD 163296 & $5-08-86$ & 108838 & 60 & ** \\
\hline $04-6 q$ & HD 163296 & $6-08-86$ & 328849 & 480 & \\
\hline 04-01 & HD 163296 & $6-08-86$ & 328851 & 150 & \\
\hline 04-01 & HD 163296 & $6-08-86$ & 108844 & 49.8 & $* *$ \\
\hline $04-6 \mathrm{~s}$ & HD 163296 & $7-08-86$ & 328855 & 480 & \\
\hline $04-6 \mathrm{t}$ & HD 163296 & $8-08-86$ & 328866 & 480 & \\
\hline $04-7 \mathrm{a}$ & HD 163296 & $7-10-87$ & 332016 & 480 & \\
\hline $04-7 \mathrm{a}$ & HD 163296 & $7-10-87$ & 111813 & 22.8 & ** \\
\hline $04-7 b$ & HD 163296 & $7-10-87$ & 332017 & 960 & \\
\hline $04-7 \mathrm{c}$ & HD 163296 & $7-10-87$ & 332020 & 480 & \\
\hline $04-7 d$ & HD 163296 & 7-10-87 & 332021 & 1080 & \\
\hline $04-7 \mathrm{e}$ & HD 163296 & $8-10-87$ & 332025 & 960 & \\
\hline $04-7 \mathrm{f}$ & HD 163296 & $8-10-87$ & 332026 & 480 & \\
\hline $04-7 \mathrm{~g}$ & HD 163296 & $8-10-87$ & 332028 & 480 & \\
\hline $04-7 \mathrm{~h}$ & HD 163296 & $8-10-87$ & 332029 & 1080 & \\
\hline $04-7 \mathrm{i}$ & HD 163296 & $9-10-87$ & 332036 & 960 & \\
\hline $04-7 j$ & HD 163296 & $9-10-87$ & 332037 & 480 & \\
\hline $04-7 \mathrm{k}$ & HD 163296 & $9-10-87$ & 332038 & 1080 & \\
\hline 04-71 & HD 163296 & $10-10-87$ & 332052 & 480 & \\
\hline $04-7 \mathrm{~m}$ & HD 163296 & $10-10-87$ & 332054 & 960 & \\
\hline $04-7 \mathrm{n}$ & HD 163296 & $10-10-87$ & 332058 & 480 & \\
\hline $04-7$ o & HD 163296 & $10-10-87$ & 332059 & 1080 & \\
\hline $04-7 p$ & HD 163296 & $11-10-87$ & 332063 & 480 & \\
\hline $04-7 \mathrm{p}$ & HD 163296 & $11-10-87$ & 111854 & 22.8 & $* *$ \\
\hline $04-7 \mathrm{q}$ & HD 163296 & $11-10-87$ & 332064 & 960 & \\
\hline $04-7 \mathrm{r}$ & HD 163296 & $11-10-87$ & 332068 & 480 & \\
\hline $04-7 \mathrm{~s}$ & HD 163296 & $11-10-87$ & 332069 & 1080 & \\
\hline $04-7 \mathrm{t}$ & HD 163296 & $12-10-87$ & 332079 & 480 & \\
\hline $04-7 \mathrm{u}$ & HD 163296 & $12-10-87$ & 332080 & 1080 & \\
\hline
\end{tabular}


P. F. C. Blondel and H. R. E. Tjin A Djie: Modeling of PMS Ae/Fe stars using UV spectra, Online Material p 30

Table F.6. Comments and notes to the Tables $1-5$. The first number of the image denotes IUE camera: $3=$ SWP, $2=$ LWR, $1=$ LWP, images not observed on the same run, but combined as they have acomparable magnitude, are marked with $(*)$. The "notes" refer to images used to complete the spectrum and applied flux normalisation factors.

\begin{tabular}{cc}
\hline \hline (1) & LWP LAP replaced with SAP starting at $2600 \AA$ \\
(2) & SWP LAP replaced with SAP starting at $1700 \AA$ \\
(3) & LWP30188 upto $2375 \AA$ followed by LWP30354 \\
upto $2565 \AA$ and completed with LWP17586 \\
(4) LWP16704 upto $2618.239 \AA$ followed by LWP16705 \\
(5) LWP30679 LAP replaced by SAP from 2500 to $3060 \AA$ \\
\hline
\end{tabular}

Appendix G: IUE images - Comparison stars.

Table G.1. B stars, HD 202850 is $\sigma$ Cyg.

\begin{tabular}{|c|c|c|c|c|c|c|}
\hline HD & & \multicolumn{2}{|c|}{$\overline{\overline{I U E}}$ images } & $\overline{\mathrm{ST}}$ & $\overline{M_{v}}$ & $T_{\text {eff }}$ \\
\hline 58350 & + & 308199 & 207127 & B5 Ia & $\begin{array}{l}-7.0 \\
\end{array}$ & 13600 \\
\hline 86440 & + & 309513 & 208239 & B5 $\mathrm{Ib}^{1}$ & -5.4 & 13600 \\
\hline 164353 & + & 310172 & 208836 & B5 $\mathrm{Ib}^{2}$ & -5.4 & 13600 \\
\hline 22928 & $\mathrm{~h}$ & 318889 & 215103 & B5 III & -2.2 & 15000 \\
\hline 83754 & $\mathrm{~h}$ & 318884 & 214903 & B5 V & -1.2 & 15400 \\
\hline 23302 & $\mathrm{~h}$ & 307921 & 206899 & B6 III & -1.85 & 14100 \\
\hline 90994 & + & 309219 & 207975 & B6 V & -0.9 & 14000 \\
\hline 183143 & $\mathrm{~h}$ & 306550 & 205659 & B7 Ia & -7.1 & 12200 \\
\hline 23630 & + & 308147 & 207078 & B7 III & -1.5 & 13200 \\
\hline 21071 & $\mathrm{~h}$ & 314767 & 211334 & B7 v & -0.6 & 13000 \\
\hline 166937 & $\mathrm{~h}$ & 309111 & 207849 & B8 Ia & -7.1 & 11200 \\
\hline 12301 & $\mathrm{~h}$ & 307282 & 206276 & B8 Ib & -5.2 & 11200 \\
\hline 23850 & + & 311245 & 209867 & B8 III & -1.2 & 12400 \\
\hline 23324 & + & 307922 & 206900 & B8 V & -0.25 & 11900 \\
\hline 21291 & $\mathrm{~h}$ & 307280 & 206274 & B9 Ia & -7.1 & 10300 \\
\hline 202850 & w & 315099 & 211614 & B9 Iab & -6.2 & 10300 \\
\hline 212593 & $\mathrm{x}$ & 333852 & 113556 & B9 $\mathrm{Iab}^{2}$ & -6.2 & 10300 \\
\hline 149212 & $\mathrm{~h}$ & 306474 & 205555 & B9 III & -0.6 & 11000 \\
\hline 108767 & $\mathrm{~h}$ & 318885 & 214904 & B9 V & +0.2 & 10500 \\
\hline 186882 & w & 309596 & 208354 & B9.5 III & -0.3 & 10500 \\
\hline 232121 & & 302249 & - & B7 III & & SX Cas \\
\hline 5679 & & 309083 & - & B7 III & & U Cep \\
\hline
\end{tabular}

Table G.2. A stars

\begin{tabular}{rllllll}
\hline \hline \multicolumn{1}{c}{ HD } & \multicolumn{7}{c}{ IUE images } & ST & M $_{\mathrm{v}}$ & $\mathrm{T}_{\text {eff }}$ \\
\hline 87737 & h & 308565 & 207305 & A0 Ib & -5.2 & 9730 \\
111775 & + & 309515 & 208241 & A0 IV & +0.3 & 9915 \\
103287 & + & 308198 & 207124 & A0 V & +0.65 & 9520 \\
156208 & h & 306777 & 205787 & A1 III & +0.2 & 9480 \\
29646 & h & 311152 & 209784 & A1 V & +1.0 & 9230 \\
197345 & + & 309133 & 207864 & A2 Ia & -7.2 & 9080 \\
137422 & h & 316447 & 212690 & A2 II & -2.9 & 9040 \\
20346 & h & 306816 & 205800 & A2 III & +0.3 & 9000 \\
48250 & h & 306496 & 205570 & A2 V & +1.3 & 8970 \\
210221 & h & 318683 & 214745 & A3 Ib & -5.2 & 8770 \\
122408 & w & 309516 & 208242 & A3 III & +0.5 & 8600 \\
216956 & + & 309134 & 207865 & A3 v & +1.5 & 8720 \\
17138 & h & 320735 & 216634 & A3 v & +1.5 & 8720 \\
5448 & h & 318568 & 214643 & A4 V & +1.7 & 8460 \\
59612 & + & 315234 & 211748 & A5 I & -5.1 & 8510 \\
189849 & h & 308030 & 206989 & A5 III & +0.7 & 8100 \\
159561 & + & 317411 & 214482 & A5 III & +0.7 & 8100 \\
116842 & h & 310285 & 208949 & A5 v & +1.95 & 8200 \\
216701 & + & 310261 & 208927 & A5 v & +1.95 & 8200 \\
28527 & w & 319459 & 215497 & A6 IV & +1.5 & 7950 \\
85123 & x & 333670 & 213331 & A7 II & -0.9 & 7900 \\
28319 & h & 318390 & 214483 & A7 III & +1.1 & 7650 \\
76644 & + & 313938 & 210553 & A7 IV & +1.7 & 7750 \\
87696 & + & 315548 & 212028 & A7 V & +2.2 & 7850 \\
28910 & m & 350013 & 227414 & A8 V & +2.4 & 7580 \\
147547 & m & 310872 & 209560 & A9 III & +1.35 & 7300 \\
\hline
\end{tabular}

Table G.3. F stars.

\begin{tabular}{|c|c|c|c|c|c|c|}
\hline HD & & \multicolumn{2}{|c|}{ IUE images } & ST & $\mathrm{M}_{\mathrm{v}}$ & $\mathrm{T}_{\mathrm{eff}}$ \\
\hline 36673 & + & 315073 & 211601 & F0 I & -5.1 & 7700 \\
\hline 12311 & + & 311242 & 209862 & F0 III & +1.5 & 7150 \\
\hline 27176 & + & 315538 & 212009 & F0 V & +2.7 & 7200 \\
\hline 40136 & $\mathrm{~m}$ & 310286 & 206995 & F1 III & +1.6 & 7010 \\
\hline 74180 & $\mathrm{~h}$ & 311051 & 202337 & F2 I & -5.1 & 7350 \\
\hline 17584 & $\mathrm{w}$ & 319465 & 215499 & F2 IIII $^{1}$ & +1.7 & 6870 \\
\hline 89025 & + & 315536 & 209732 & F2 III & +1.7 & 6870 \\
\hline 40136 & + & 310286 & 209364 & $\mathrm{~F} 2 \mathrm{~V}$ & +3.6 & 6890 \\
\hline 99028 & + & 311311 & 209918 & F3 III ${ }^{1}$ & +1.7 & 6737 \\
\hline 78362 & $\mathrm{~h}$ & 308032 & 206991 & F3 III ${ }^{2}$ & +1.7 & 6737 \\
\hline 164259 & $\mathrm{x}$ & - & 115556 & F3 V & +3.6 & 6740 \\
\hline 185395 & $\mathrm{~m}$ & 340443 & 119464 & $\mathrm{~F} 4 \mathrm{~V}^{1}$ & +3.55 & 6665 \\
\hline 26462 & $\mathrm{~m}$ & 349838 & 127243 & $\mathrm{~F} 4 \mathrm{~V}^{2}$ & +3.55 & 6665 \\
\hline 20902 & + & 315316 & 207094 & F5 Ib & -6.6 & 6900 \\
\hline 27524 & W & 304756 & 204119 & F5 V & +3.5 & 6440 \\
\hline 160365 & $\mathrm{w}$ & 316491 & 204122 & F6 III & +1.6 & 6363 \\
\hline 82328 & $\mathrm{w}$ & 319466 & 215500 & F6 IV & +2.5 & 6360 \\
\hline 173667 & + & 310784 & 209459 & F6 V & +3.6 & 6360 \\
\hline 102870 & + & 307305 & 204867 & F9 V & +4.2 & 6115 \\
\hline
\end{tabular}

Table G.4. G stars.

\begin{tabular}{|c|c|c|c|c|c|c|}
\hline \multicolumn{2}{|l|}{$\overline{\mathrm{HDD}}$} & \multicolumn{2}{|c|}{ IUE images } & ST & $\mathrm{M}_{\mathrm{v}}$ & $\mathrm{T}_{\mathrm{eff}}$ \\
\hline 159181 & $\mathrm{~W}$ & 302348 & 204124 & G2 II & -2.3 & 5250 \\
\hline 2151 & w & 307429 & 204125 & G2 IV & +3.0 & 5655 \\
\hline 10307 & w & 310029 & 204854 & $\mathrm{G} 2 \mathrm{~V}$ & +4.7 & 5860 \\
\hline 161797 & w & 303652 & 204121 & G5 IV & +3.1 & 5460 \\
\hline 20630 & + & 309462 & 204857 & G5 v & +5.1 & 5770 \\
\hline
\end{tabular}


P. F. C. Blondel and H. R. E. Tjin A Djie: Modeling of PMS Ae/Fe stars using UV spectra, Online Material p 31

Table G.5. K stars.

\begin{tabular}{|c|c|c|c|c|c|}
\hline$\overline{\mathrm{HD}}$ & & IUE images & $\overline{\mathrm{ST}}$ & $\overline{\overline{\mathrm{M}_{\mathrm{v}}}}$ & $\overline{\overline{T_{\text {eff }}}}$ \\
\hline 62509 & $\mathrm{w}$ & $\begin{array}{lll}304730 & 209844\end{array}$ & K0 III & +0.7 & 4750 \\
\hline 198149 & $\mathrm{w}$ & - 212739 & K0 IV & +3.1 & 5000 \\
\hline 185144 & $\mathrm{w}$ & -205989 & $\mathrm{~K} 0 \mathrm{~V}$ & +5.9 & 5250 \\
\hline 4128 & $\mathrm{w}$ & -212181 & K1 III & +0.6 & 4600 \\
\hline 142980 & $\mathrm{~m}$ & $207617+207619$ & K1 IV & +3.1 & 4840 \\
\hline 10476 & $\mathrm{w}$ & - 212041 & $\mathrm{~K} 1 \mathrm{~V}$ & +6.1 & 5080 \\
\hline 85503 & $\mathrm{w}$ & -209857 & $\mathrm{~K} 2 \mathrm{III}^{1}$ & +0.5 & 4420 \\
\hline 137759 & $\mathrm{w}$ & -209858 & $\mathrm{~K} 2 \mathrm{III}^{2}$ & +0.5 & 4420 \\
\hline 22049 & $\mathrm{w}$ & -212671 & $\mathrm{~K} 2 \mathrm{~V}$ & +6.4 & 4900 \\
\hline
\end{tabular}

Table G.6. References to Col. 2 .

\begin{aligned} \hline \hline w & Wu C.C., Ake B., Boggess A., et al. 1983 IUE NASA \\ & newsletter 22 \\ h & Heck A., Egret D., Jaschek M., Jaschek C., 1984 ESA \\ & SP-1052 \\ + & Wu 1983 \& Heck 1984 \\ x & Wu C.C., Crenshaw D.M., Blackwell jr. J.H., et al. 1991 \\ & IUE NASA newsletter 43 \\ m & Wu C.C., Mo J., Crenshaw D.M., 2000 \\ & A Complete UV Atlas of Standard Stars \\ & http://www-int.stsci.edu/ jinger/iweb/proj/ \\ & project.html \end{aligned}

